
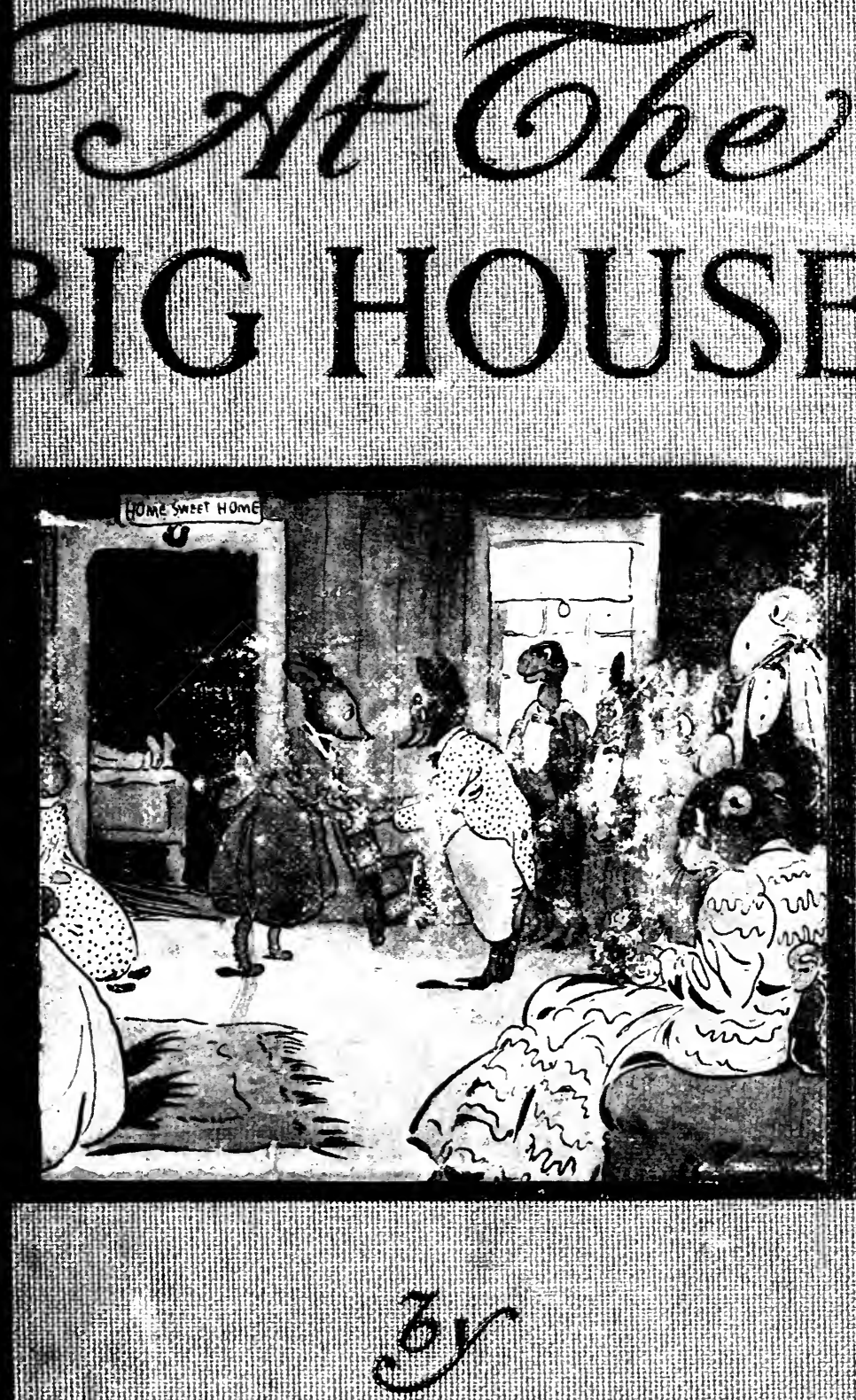


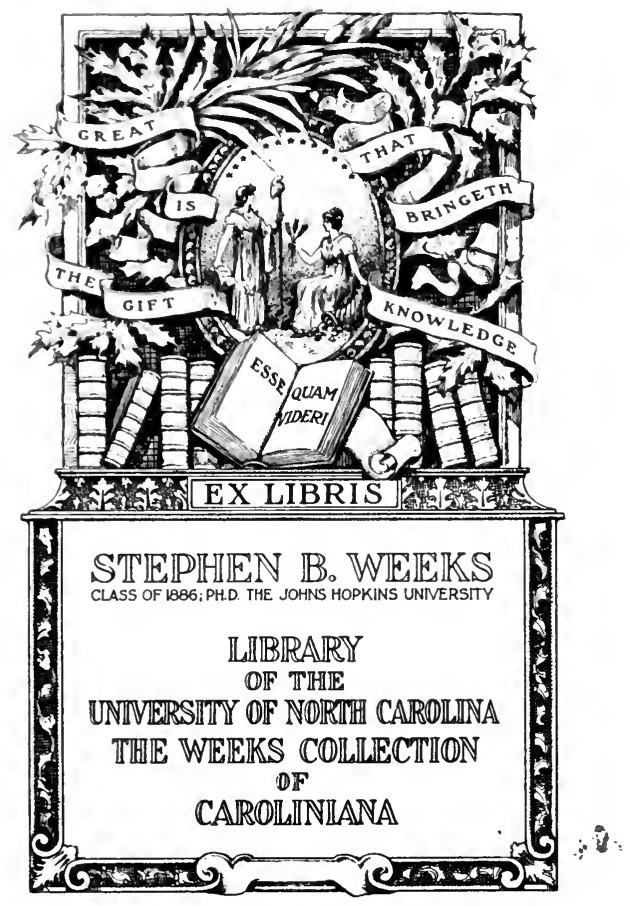


This book must not be taken from the Library building. 

A'T THE BIG HOCSE 




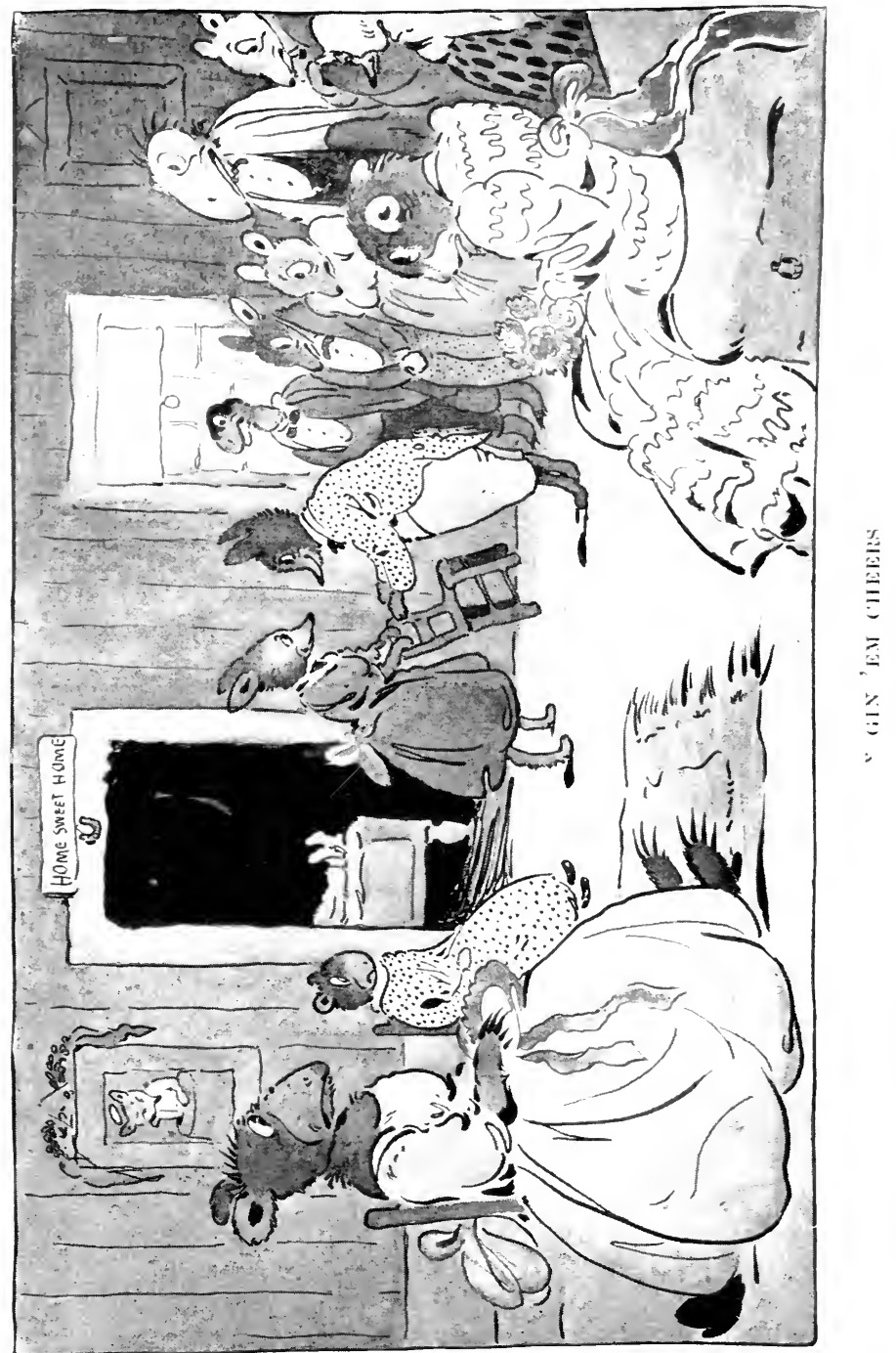




\section{A'T THE \\ BIG HOUSE}

Where Aunt Nancy and Aunt'Phrony

Held Forth on the Animal Folks

$B y$
ANYE VIRGINIA CULBERTSON

Illustrated by

E. WARDE BLAISDELL

INDIANAPOLIS

THE BOBBS-MERRILL COMPANY

PUBLISHERS 
('OPYRIAHT 1904

Thy Bobbs-Merrill Company

April

PRESS OF

BRAUNWORTH \& $C O$.

BOOKBINDERS AND PRINTERS

BROOKLYN, N. Y. 


\section{CONTENTS}

Going Down to Uncle Henry's

PAGE

Mr. Fox's Funeral

Mr. Fox and Molly Hare Go Fishing

Negro 6

Mr. Hare Imitates Mr. Bear

Negro 15

The Friendship of Mr. Bear and Mis' Turkey

Indian 26

The Toad, the Grasshopper and the Rooster

Indian 34

Mr. Terrapin Gets the Nose-bleed

Tegro 41

How the 'Possum's Tail Became Bare

Negro 49

Why the 'Possum Has Black Ears

Indian 56

How Mr. Terrapin Lost His Beard

Indian 63

How Mr. Terrapin Lost His Plumage and Whistle

Indian 70

Mis' Cat and Mr. Frisky Mouse

Indian 78

Mr. Hare Tries to Get a Wife

Negro 86

How Mr. Hare Lost IIis Horns

Indian 93

Why the Titmouse IIas a Blunt Tongue

Indian 100

The Woman Who Married an $O w l$

Indian 106

Mr. Mud-turtle's Adrenture

Indian 115

Why the Flounder is Flat

Indian 122

Brother Squirrel and Molly Hare

Negro 19

Mr. IIare and Mr. Flint Rock

Negro 135

Mr. Wildeat Goes Turkey-hunting

Indian 141

Ir. Fox Turns Farmer

Indian 148

Canarli and the Wolves

Negro 155

The Story of a Giant

Indian 164

Why the Buzzard is Bald

Indien 169

Indian 177 


\section{CONTENTS}

Mis' Goose Deceives Mr. Bear

PAGE

Mr. Bear Tends Store for Mr. Fox

Negro 186

Negro 194

A Star Story

Indian 201

Why Crabs Walk Backward

Negro 207

The Origin of the Cat

Negro 215

The Dragon and the Thunder

Indian 222

The Tlaniwa

Indian 227

The 'Possum and the Grub-worm

Indian 232

Mr. Bear and Mr. Terrapin Go Courting

Negro $23 \overline{7}$

Molly Cotton-tail Steals Mr. Fox's Butter

Negro 245

The Lazy Fox

Negro 253

Mr. Hare, Mr. Mink and Mis' Duck

Indian 260

The Humming-bird and the Tobaceo

Why Moles Have Hands

Indian 268

Negro 273

The Fox and the Duck

Negro 279

Why the Ground-hog Has a Short Tail

Indian 284

Mr. Hare, Mr. Wilikat and Mr. Otter

Indian 290

Mr. Hare and Mr. Elephant

Negro 297

The Toad and the Terrapin

The Mocking-bird and the Dry-fly

Negro 302

Negro 308

The Saucy Young Frog

Negro 314

The Crane and the Humming-bird

Indian 321

The Fox and the Hot Potatoes

The Funeral of Mr. Dog

How the Deer Lost His Upper Teeth

Negro 327

Negro 333

Indian 338

The Hare Disappears For Ever

Indian 342 


\section{INTRODUCTION}

The stories presented in this rolume were found chiefly among the negroes of southeastern Virgini: and the Cherokee Indians of North Carolina. It is possible that a few of the Indian stories belong to the Creeks, as some of them were obtained from an Indian who, while living among the cherokees, was said by them to be a Creek; but this was a point on which he declined to commit himself. In the mountains of the extreme west of North Carolina the writer listened to stories wonderfully like thoze of "Lncle Remus," falling from the lips of venerable Indians with a strange effect of familiarity. Here was "the Rabbit," the same lively, merry, tricksy, resourceful, quite reprehensible and utterly irresistible fellow as in Mr. Harris' delightful negro tales. It seems odd that the two races, so different in temperament and characteristics, should assume exactly the same attitude, distinctly a humorous one, toward the timorous rabbit, perhaps the scariest "li"l fool creetur" of them all, and glorify him as the most valiant of heroes. It may be somewhat easier to understand in the light of the fact that the rabbit was an important hero-god in the mythologies of both races.

Both the Indian and the negro stories are presented in negro dialect in order more strongly to emphasize the resemblance between them-so marked as to give 


\section{INTRODLCTION}

rise to the supposition that one race borrowed from the other, though which, in that case, was originator and which borrower it would be difficult to say. Scientists, for the most part, believe each race to have originated its own stories, while others are of the opinion that all such tales were born in the morning of time in some common cradle of our kind.

The reader will have no difficulty in separating the two sets of stories. The source of each is noted in the table of contents, and, moreorer, those of Indian origin are all told by an elderly negress. Aunt 'Phrony, who is supposed to be Indian on the father's side and negro on the mother's. - a not unusual admixture of race in the old dars of the South; while the negro tales are told by a woman of purely African descent, old Aunt Nancy. In the case of the Indian stories, it has been thought not inconsistent with the unities to give them a large flavor of negro life and character, since a halfbreed, such as the one imagined, would have been, by law, a slave like her mother, brought up amongst the negroes and partaking of their ways, speech and characteristics.

Let no one suppose that Indians have not a sense of humor. They laughed heartily as they told these stories and were visibly disappointed if their auditors failed to catch the point and to laugh with them. It may be of interest to observe how in the story of "Мrr. Mud-turtle's Adrenture" the Indian has made the turtle effect his escape by exactly the same ruse as that practised by the rabbit in the famous "Tar Baby" story.

A point of difference noticed by the writer was that in the Indian stories of which the rabbit was the hero 


\section{INTRODTCTION}

he was always represented to be of the male persuasion, while in the negro tales of the same class the chief actor was usually "Ol' Molly Hyar,", or "Mis' Hyar", or "Mis' Molly Cotton-tail." In fact, the negroes of Virginia seldom refer to "the rabbit"; almost always it is "ol" hyar"."

The negro has borrowed some stories from Esop"s fables and adapted them to suit himself. An example is given in this volume in the story of "The Fox and the Hot Potatoes," plainly an adaptation of the famous story of the cat's paw, the monkey and the roasted chestnuts. The writer found a number of others, including "The Fox and the Grapes," "Fox and Rooster," and "The Sick Lion."

In conclusion it should be said that these stories were all collected from persons well on in years, unable to read and without opportunity of access to books. They are confessedly "edited," for all who have collected folk-tales will know the crude form in which they are obtained, usually a bare, brief outline, though now and then one falls in with a genuine raconteur. The aim has been to imitate, as far as possible, the style of the latter, while jealously preserving the original outlines, so as not to impair their value as folk-lore. To those who would study the imagination of primitive peoples these stories should have some ralue, if for no other reason than that they add a few more to the stock of this class, the opportunities for gathering which grow less and less with each rear and soon will cease altogether.

Axye Virginia Cllbertson. 

A'T THE BIG HOCSE 



\section{AT THE BIG HOUSE}

\section{GOING DOWN TO UNCLE IIENRY'S}

The three children, Ned of ten, Janey of eight, and Kit, the yellow-haired laddie of fire, were wild with delight at the prospect of a risit to their uncle on the old plantation where their mother had been reared; for little Kit had never been there, and the others were too young to recollect a former visit. Their mother had married and gone North to live some time before the Civil War. Then came the troulbled years of strife, when it was unsafe for her to renture with her little brood into the war-srept. storm-beaten section of southern Virginia where her old home was situated. Now, however, the struggle had been over for a year, and she felt a great longing to see home once more, to know what familiar and cherished objects had survived the wreckage of war, and to give her children a glimpse of the old order of things before it should disappear for ever. She had told them so much of the place and of her life there, that they felt as if they were going to risit some well-known spot, and in particular were filled with lireliest anticipations of hearing for themselves, and in the proper surroundings, the old darky songs and tales of which she had tried to give them some idea. 


\section{AT THE BIG HoUsE}

The days rolled around all too slowly for the impatient little people, but at last they were off. First came the railroad trip, then the night ride on a steamer down the great bay. and in the morning another railway journey, this time through pine woods for the most part. on a little narrow-gage train whose engine secmed to be picking and choosing its path and getting out of the way of the trees as it zigzagged in and out between them.

They were met at the rude little station by a wagon and a pair of stout farm-horses, instead of the carriage and blooded pair of other days. The children piled in on the straw at the bottom of the wagon, and thought it great fun to dodge the uncertain morements of the trunks as they jolted along orer a rather rough country road, which took them for miles through the tall, slim, fragrant pines that looked so dark and somber when viewed from a distance, but which nerertheless let in plentiful rays of sunlight to flicker and dance over the soft carpet of brown needles beneath.

It was almost sunset before they came in sight of the house, which was wholly unpretentious but entirely comfortable, as was the case with many a plantation home of the old dars. Situated on a slight eminence, it was surrounded by a huge open expanse whose only boundary was a far, dark rim of pines.

In the center of the lawn was a huge oak tree, a perfect giant of his race, which had looked benignantly down on the same family for a hundred and fifty years, old when the founder bought the land from the Indians, yet still full of life and vigor, bidding fair to remain through many another generation of the puny 


\section{GOING DOWN TO UNCLE HENRY'S}

race of men. He towered so high above everything else that he was first to wave a stately salute to the rising sun and last to nod a grave good-night as the beams disappeared behind the pine trees. The morning rays always found him, like some benevolent patriarch, giving shelter to a little company of cows and sheep and snow-white geese; and as the sun slipped gradually around his trunk to its goal in the west, there was never an hour of the day in which his shady hospitality was not claimed by some living creature, while the shadow of his trunk marked the flight of time like some huge sun-dial whose face was half the lawn. It was impossible to regard him as a mere tree; he made you think of a rigorous, "grand old man", and after you had known him a while, he seemed like an important member of the family.

Dotted about the lawn were the rarious "offices," and farther back, the "quarters", where a hundred or more negroes used to be lodged in the old, busy days, when everything was manufactured on the plantation, from linsey-woolsey to cart-wheels, and everything needful raised, even the indigo for dreing the home-made cloth.

As the wagon jolted across the lawn, the children stood up and waved their handkerchiefs to the little party assembled in the front porch. Waiting to receive them stood Incle Henry, tall, broak-shouldered, cheery and hearty. There, too, wére the dogs, barking and capering and giving noisy welcome, and a little in the background was one whom ther could not fail to recognize, so often had they heard her described,-a fat, smiling, broad-faced negress, comfortable and sleek, with eyes beaming and white teeth shining, Aunt 


\section{I'T THE BIG HOUSE}

Taney, their mother"s old "mammy". The children were lifted down amid huggings and kissings from Tncle Ilenry, fawning and frolicking from the dogs, and lastly Aunt Nancy fell on them and took them to her ample bosom and made much ado over her "young Miss" dhillen".

Presently the servants began ruming to and fro carrying steaming dishes from the "cook-house" to the "hig homse". and before long the children were seated about the fine old mahogany table, which had dispensed howitality to at least four generations, and were eagerly discussing hoe-cake and dodger, "egg-bread" and mutfin, waflle and fried chicken, together with other staple delicacies, familiar and unfamiliar. They were waited upon, meanwhile, by "Coonie", the house-boy, son of Eliza, the cook, who watched every monthful taken with absorbed interest, and grimned as if each new depredation on the piled-up plates was a fresh compliment to his mother's skill in cookery.

When supper was over and the children were being put to bed. Aunt Nancy's smiling face appeared in the doorway, and she declared that she must have "anu "rr squint at dem chillen" before she took herself off for the night. "Blessid lambs!" said she, "de ve'y spit an' imidge er dey maw, 'scusen de li'l bit whar favers dey gran'paw an' ol Mis' an' dey Uncle Hinry. Dellaws! I nurer think in de days w'en I tucken Miss Janey on my knee an' got her raidy fer baid, an' den tnck her in an' sot down by her an' tell her tales ontwel she drap off ter sleep. I nurer think, naw ma'am. dat I gwine live ter see her wid a hull passel er li'l chillen husse'f, naw'm, dat I didn'." 


\section{GOING DOWN TO TNCLE HENRT'S}

At the mention of stories the children sat up in bed, clasping their arms about their knees with chins resting on top, looking eagerly at her, like so many animated interrogation points. "Please tell us some stories," they begged, and Janey declared that she did not believe she could possibly go to sleep in a strange bed, unless, as her mother used to do, she fell asleep on a story.

Aunt Nancy giggled and laughed until her body rocked from side to side. "Well, ef dis ain" de beatenes' lot." said she. "ter "mence on me bout tales de re"y fus' night I sot eyes on 'em. Jes" zackly de way dey maw useter kyar" on, fer all de worl"." But it did not take much coaxing to start her. Drawing up an old splintbottomed chair, she sat down between the two beds and announced that she beliered she would "splunge inter de bizness" by telling them the story of 


\section{MIR. FOX'S FUNERAL}

"In de ol' dars," she began, "dar wuz two er de creeturs whar wuz alluz fallin" out wid one mu'rr, an' seein' who kin git ahaid ur tu'rr one, an' settim' all sawts er traps an' lay-overs-fer-ter-ketch-meddlers. Dese two creeturs wuz neener mo' ner less dan Mis' Molly Cotton-tail-w ich some calls 'er ol' MIolly Hyar-an' Mistah Slickry Sly-fox. Sometimes one wuz in de lead, sometimes tu'rr, but mos' in gin'ly Mis' Molly she camed out ahaid, fer dat seem ter be de speshul gif' er de ladiz, ter git der own way wid der breens stidder dey fistes. Menfolks is kind er clumsy an' lumbersome 'bout sech ez dat, an' mos' times gins derse'fs erway fo' dey gits half thu.

"One day he come 'crost 'er w'en she'z right good an' tired, settin' in de broom-saidge fiel', down by de ol' sawmill, an' he gin 'er chase thu de woods an' inter de swamp an' out inter de fiel' on tu'rr side, 'twel she wuz all blown an' clean store-up. 'Oh me! O my!' sez she ter husse'f, sez she, 'I reckon dish yer's whar I gotter turn up my li'l toes an' gin up de ghos', sho' 'nuff 'kase I kain't run nu'rr step, no use ter try. Well, I hope some nice nigger man gwine git dish yer lef. behime foot er mine an' kyar' hit roun' wid him ter keep de boogers off." "'

Here the children interrupted to make inquirie 


\section{MIR. FON'S FLNERAL}

about the rabbit's left hind foot, of whose wonderful powers they never had happened to hear.

"Dellaws!" exclaimed Aunt Nancr, "nurer rearn tell er de lef" behime foot ur a grave-yard rabbit: "Whar you been livin' all dese ears? It'y dat's de mos" portul cunjer in de worl. Jes" krar" one'r dem in yo pockit an' rouse safe ez ynu kin be in dish yer sufferin. dyin worl: Hit keeps off witches an ha'nts an jacky-mylantums an' boogers in gin'l. an' hit brings good luck an' keeps away bad luck. an' hit keeps mean. low-down folks f'um puttin' spells on you an trickin you. But hit wh'ks bofe ways, fer ef you lose dat foot an some one else gits hit, dey kin do jes" bout wat dey want: wid you. A An' you mus'n on no krount let no pusson teck dat rabbit foot in han' an' tetch you wid hit, fer ef you do, sump'n mighty bad gwine happen to you, dat's jes' ez sho' ez I'm a-settin' yer runnin' on 'bout dat cunjer.

"Now whar wuz I at? Oh, yas, whar Mis' Molly wuz all tuckered out an' 'bout ter gin up de game. Well, jes" den, ez de luck had hit, she yearn a gre"t blowin" er hawns an' a lot er houn's girin' tongue a fur ways off, an' she knowed dat de hunters wuz som'ers roun', so she ga'rrd husse'f toge'rr an' putt out in dat d'reckshun, an bless goodness of she ain tole ol' Fox right in de midse er de dogs an' de hosses an' de hunters, an' den she double an' git outen de way in shawt order. I tell rou. Mistah Slickry Sly had a mighty clost share dat time, an' he ain' fergive Mis' Molly. He laid hit up in his min' erg"in 'er an' 'clared he wuz gwine git even wid her ef he ain' do anu'rr lick dat winter.

"He set by de fire an' study an' study 'bout hit, wid 


\section{AT TIIE BIG IIOTSE}

his haid on his han' an his jaw drapt open, twel he wot sort or rum down an his appentite gin out. Mis' Fox she wuz worrited bout him, same time dat she wuz putt out wid him fer settin' on his hanches doin' muttin' an' lettin' her dus' roun' atter de vittles. Las', one day she tucken de broom an' shuk hit at 'im, an' sez she, 'Git outen my sight, you mis able shif'less creetur. Ef you go an stir yo'se'f rom an' wu'k a li'l. hit mought start yo' blood ter goin erg“in, an’ kyore you, fer you done got a bone disease, w'ich dey calls hit lasy-bones, an I'm a-gwineter kyore you right yer an' now, dat I is, fer I ain' want no fun'l 'bout dis house, dat I ain', wid all de strouds an' de coffins an' de flow's an' de hearsts; an' de vittles whar de mo'ners 'stroy at de settin'-up. Cos' me mo' dan you uver been wuf, suh.' An' wid dat she brought de broom, ker-smack! down on Mistah Sly-fox's haid.

"W'en she say de wu'd 'fun'l,' dat gin 'im a idee. He laid dar widout movin', lak he wuz sho'-'nuff daid, an' he let her pick 'im up 'an putt 'im on de baid. He laid dar a w'ile lis'nin' at 'er go on, wringin' her han's an' crrin' 'O lawd: O lawd! w'at a wicked ooman I is! Done kilt my po' sick husban'! W'at I gwine do! Oh, mussy me. wat is I gwine do!"

"Fox he wuz might"ly tickled an' let 'er run on a w'ile. He say ter hisse'f, 'Dish yer's whar I fin' out how de ol ooman gwine 'have husse'f w'en she's a widdy.' Den he open he eyes lak he wuz pow'ful fibble an' roll 'em up in his haid, an' sezee: 'Ol' ooman. I fergives you fer dis, dleed I does. I kain't 'spec ter las' much longer: lemme ax you, befo' I goes, ter gimme a decint fun'l, wid all de fixin's. an' I want a sarmint preached 


\section{MR. FON'S FLNERAL}

fer me, too, ef you hatter putt off de preachin part fer a 'ear. so"'t you kin pay fer hit. An" I wants you, please ma`am. ter "vite all de nabers ter de settin'-up, even Mis' Molly Cotton-tail, kase I done fergive her, too, an you mus" sen her wu'd dat I gwine lay mo" peace"ble in my grare ef she"ll come ter de settin-up. An' I wants you ter have plenty er vittles fer de moners, 'kase I ain' want no pusson ter go 'way f'um my fun'l an' say he's hongry.'

"Mis' Fox she jes' lit out inter cryin' an' wringin' her han's erg"in, but she gin de promus. She say, sez she: 'Ol' man, you kin die easy, fer I gin you de w'd ur a po' widdy ooman dat you is gwine have er'ything dat b"longs wid a fus'-class fun'l, all de trimmin's an" de fixin's thowed in, ef I hatter w w $\mathrm{my}$ fing-ers ter de bone over de wash-tub ter pay fer "em, you is so. Don' you let dat idee keep you ling in " on yer in tawment; I done gin you my w' d, an' dat orter be 'nuff ter let you down inter de grave on flow'y baids ur ease, 'deed hit ort.'

"Fox he thanked her, an' den he fetched a big groan an' rolled orer on his back an turnt his toes up in de air an' lay dar ez stiff an' start ez ef de bref er life clean gone outen him. Den Mis" Fox she wipe her eyes on her sleere an' whu'l in an' git er'ything raidy fer de settin'-up. She kill a chicken an' bile a ham an' cook a mess er greens, an' den she tuck an' sont noration ter de nabers ter come ter de settin'-up. Den she turn in an' fix up de house, an' las' ur all she gin her 'tention ter de cawpse, an' w'en she git thu wid 'im he sut'n'y look mo' harnsum dan he done w'en he wuz walkin" thu dis vale er tears. She sont off fer de coffin an de 


\section{AT THE BIG HOUSE}

flow's, an' w'en de mo'ners got dar ter do de settin' up, erything wuz good an raidy.

"Well, she gin 'em a good bait er vittles, an' dey sot up endurin' er de night a-mo`nin' an' a-groanin' an' a-lronin', an' er'y onct in a w'iles de widdy 'ud th'ow her ap 'un over her haid an' bus' inter tears an' rock back an fo th an kyar" on twel some er de men-folks 'ud come an' console wid 'er, an' den she'd pick husse'f up a li"l. Slickry he kep' one ere an' one year open, an' he ketehed her runnin' on wid Mistah Coon a li'l an' lookin' at 'im mighty sweet w'en she see de res' wan't lookin'. 'Lh-huh!' sezee ter hisse'f, 'dat's how de win' blow, do hit? 'Tain' gin ter er'y man ter see w'at kind er widdy he gwine leave behime. Runnin' on wid ol' Coon right befo' my face an' eyes! Well ef I don' pay her off fer dat, my name ain' Slickry Slyfox. Widdy indeed! Tot fer long, ef she have de sesso.' An' 'twuz all he cu'd do not ter git up right den an' dar an' pick a quo"il wid "er.

"All thu de night de mo'ners kep' hit up, rockin' back an' fo'th an' singin' lak dis, m-um-ah-um-m, m-um-ah-um-m."

Here Aunt Tancy imitated the peculiarly mournful, monotonous dirge indulged in by the negroes at their "settin'-ups," consisting of only a few notes without words, hummed through closed lips, and wailed with such persistent dolefulness through the long night that the effect is indescribably harrowing as well as melancholy. Presently she resumed her story.

"Yas, dey kep' hit up all night. dough now an' den dey'd stop fer a li'l set-to wid de rittles. W'en de mawnin' come, all un 'em 'scusin' one er two er de wimmin- 


\section{MR. FOX'S FUNERAL}

folks went home ter dress fer de fun'l. Long to'des twelve, yer dey comes ag "in, dress up in all de fine doin's dey kin lay der han's on. I 'spec' ol' Mis' B'ar wuz 'bout de fines' one, she have on a pink silk dress, lowneck-an'-shawt-sleeres, wid a trail an' a pink sunshade ter match, but Mis' Panter run 'er right clost, 'kase she have on a white tall'ton wid flounctes f'um top ter bottom an' bows er raid ribbon wid streamers behime. De gemmen all have on neckties an' white cotton glores, an' raid hank'chers stickin outer der pockits. I tell you dat $w u z$ a fun'1! Dey all come in an' dey howdied a li'l wid Mis' Fox an' she telled 'em she wuz proud ter see 'em dar, an' den she gin 'em cheers an' ev'b'dy sot down.

"Las' some un say: 'I 'clar' ter gracious, Sis' Molly Cotton-tail ain' comed yit. Wunner w'at mek her so late? Any you-all seed 'er on de way yer? Reckon she done stop ter prink hussef up.' To pusson ain' seed 'er, so some 'un say dey bes' sing a chune w'ile dey 'uz waitin', an' wid dat dey struck inter Zion Weep a-Low, an' I tell you, honeys, dey made sho'-'nuff music, wid de ladiz kyar'yin' de air an' de men-folks doin' de dronin'."

Here the children again interrupted to ask Aunt Nancy if she knew Zion Weep a-Low, and to beg that they might hear how the creatures sang it at the funeral of Mr. Fox. Nothing loath, Aunt Nancy sang it with camp-meeting ferror, notes long drawn out, with many an "oh" and "ah," and shakes and quarers impossible to describe.

"Well," the old woman resumed, "dey sung dat sper'chil plumb thu an' yit Mis' Molly ain' come, so 


\section{AT THE BIG HOLSE}

dey had solemn ennelave fer a w'ile an' den de preacher he got up an' de'rl his thoat a time er two an "meneed talkin 'bout Mistah Fox. He say, 'Simner fren's, I wanster call yo" "tention ter dis eawpse; you lin see fer yo'se'fs wat a nice eawpse hit is, wid real white gloves, kiil. suh. on de han's, an' flow's strewed all up an' down 'im: an' I wants y'all ter teck p'tickler notuss er dat, fer dat gwine l'arn you how hit pars ter be hones' an' indush'ous, 'kase of he ain' been dat-a-way he oon had no sech a fun'l ez w'at dis is, wid me yer too, inter de bargum, ter gin 'im a send-off, all nice an' proper, w'ich Sis' Fox, de wife er de diseased, she a-goin' ter pay me fer hit on time, I oon keep her waitin' fer de fun'l sarmint twel nex' ear, naw suh.'

"Some er de mo`ners fetehed a groan an' some er de ol' men an' wimmin 'spon' f'um de cornders, 'Yea, lawd! hones' an' indush'ous, dat's de trufe!' an' Mis' Fox gin a squeal an' fell back in her cheer an' de fun'l hatter stop 'twel dey eu'd bring 'er to wid a go'de er water. Jes' den, who shu'd putt 'er haid in de do' but Mis' Mrolly Hyar', but she wuz too smart an' know Mistah Sliekry sly too well ter putt her foot inside de do'. She wuz all dress' off in black, wid a big bawnet, an' a mo'nin' veil mo'n a yard long streamin' down 'er back, an' she 'uz kyar'yin' a big white hank'cher wid a black bawder.

"She howdied wid 'em a li'l an' den she stan' outside an' look in at de carpse wid her haid on one side an' her mouf drord down lak she 'uz mighty 'flicted 'bout dis, an' she say, she do, moppin' her eyes now an' den wid de hank'cher, 'Po' Brer Fox, po' Brer Fox! I sut'n'y nurer 'spected ter see 'im lak dis. I done fer- 


\section{IIR. FONS FLNERAL}

give 'im all de hard feelin's dat has pass' betwix' us. He sut'n'y is a nice carpse. Sis' Fox, an' one dat you gwine be proud ur all de res' er yo' days. I has on 'y one fault ter fin' wid 'im, an' dat is, his han's ain' crost; I done yearn my granny say, an' she wuz a mighty knowin' ooman, dat de han's ur a cawpe mus" alluz be folded, look lak 'tain' a sho'-'nuff cawpse lessen de han's is crost.'

"At dat ol' Mistah Slickry Sly tuck an' slip one han' 'cross tu'rr an' laid dar lookin' ez innercent ez a lamb, but, bless yo' soul, dat 'uz 'nuff fer Miss Molly, she knowed den dat 'twuz jes' de way she 'spicioned all erlong, an' dat Slickry wuz nuver mo' erlive in his life. She jes' tucken leg-bail fer her 'scape outen dat, an' erway she go wid her mo'nin' reil streamin' out behime 'er in de win'. Fox he jumped up an' upsot de preacher an' spilt all de flow's and tuck atter 'er, hard ez he cu'd split, but he was sort er hilt back by de good clo'es an' de white kid glores, an' sidesen dat he wuz sort er stiff an' weak f'um layin' still so long. wid nuttin' ter eat inter de bargum, so he ain' see mon de een' uv 'er reil gwine roun' a cornder.

"Dey do say dat fun"l come mighty nigh mekin" trouble in de Fox fambly, fer he useter thow hit up ter de ol onman. er'y now an' den, dat she done kyar' on wid Mistah Coon right in front er de cawpse ur 'er own husban'. But she knowed how ter shet 'im up: she alluz say: "Twant no cawpse! dough hit orter bin, seein' all hit done cos' me. To 'spectable cawpse oon do no sech a low-down way, a mannerly cawpe nd 'a knowed w'at wuz 'spected ur hit an' stayed daid. An' all dem mo'ners doin' all dat mo'nin' an' settin' up fer 


\section{AT TIIE BIG IIOLSE}

nuttin’! I bom' you w'en yo' time sho'-'nuff comes I won't be able ter fin' mo'ners 'nufe in dish yer kyounty ter bury you decint. Folks ain lak ter has der feelin's disapp inted dat-a-way! Don' you talk ter me, long ez we owin' money on dat fun'l yit!" " 


\section{MR. FOX AND MOLLY HARE GO FISHING}

When Aunt Nancy had finished the tale of Mrr. Fox's funeral there were no signs of sleep in the bright eyes fixed upon her face, and three piping little roices began to make pleas for just one more story.

"G'long "way f"um yer," said she, with a chuckle, "y'all boun' ter keep me givine on all night, I sees dat. Huccome yo" eves so wide open? Atter all dat trabblement you bin doin', de San' Nan orter bin yer long 'fo' dis. Wa't you reckon yo' maw gwine say ter dis all-night bizness?"

"Oh, she won't care if we have just one more, will you, Mamma?" came in anxious chorus. "And you know, Mamma," said Janey, "you used to do this way, too; you said you did, yourself."

Namma stood in the doorway a moment, smiling and conricted. "Well, just one more, Mammy, remember, only one. Don't let them coax you for another," she said as she went downstairs to Uncle Henry.

Aunt Nancy looked as pleased as the children, but she made pretense of being completely run aground for stories, in order that she might hear the little roices raised in protest and entreaty.

"Aw, pshaw!" said Ned, "I know better'n that, for Mamma says you know enough stories to fill a book." "Well, mebbe I does an' mebbe I doesn'," said the old 


\section{AT THE BIG IIOT'SE}

woman, "but howsomurer dat may be, my min' done let "em all run ont, same 'z water thu a sieve, lessen hits one li’l one dat kind er git ketched in a comder ur my 'membance. Jes' a lïl tale bout de time w'en Mistah Fox an Mis Molly Cotton-tail went fishin toge rr.

"Atter Mis" Molly done turn de tables on "im at de fun'l. he kep" on studyin' an 'studyin' an' schemin' an' schemin ter git even wid er. Folks dat met up wid him in de woods knowed he wuz up ter sump'n', kase he went trottin' by, not stoppin' long 'nuff ter 'spon' howdy, lookin' so knowin' outen dem slant-up eyes er his'n. wid his face all drord up inter wrinkles, dat dey cu'd see he wuz plannin' out some sort er cussishness, an' dey tuck good kyare ter keep outen de way. Eren w'en he wuz foolin' de dogs, settin' up on a ol' log wid his tongue hangin' out, jes' ez still 'z ef he wuz daid, so 't dey'd pass 'im by, he wuz studyin', studyin' 'bout Mis' Molly. Same way wen he went down ter de orchud ter git "im a chickin. Mighty hard wuk ter git de chickins down f'um de tree, but still 'tain' drive Mis' Molly outen he min'.

"How does de fox git chickins outen de trees? Umph, honeys! dat sutn'y is a sight in de worl"! Ef you onct see dat you ain' gwine fergit hit in a hurry. Well, one'r dese yer cloudy nights he comes 'long un'need de tree whar de fowels is ronstin' an' fin's 'em all fas. asleep, an' he knows dat ain' gwine do, 'kase dey locks der claws tight roun' de limb an' goes ter sleep, an' de claws stay locked ontwel dey wakes up. He know he 'bleeged ter rouse 'em 'fo' he kin git 'im one. So he gins a sharp bark an' jumps up, an' w'en dey 'mence ter cackle he 'mence ter succle roun' an' roun' un'need 


\section{IIR. FOX AND MOLLY HARE GO FISHINT}

de tree, faster an' faster, jumpin' an' barkin'. De chickins dey turn an' twis' der haids ter watch 'im, an' las' some fool fowel dat's kind er weak in her hail gits so dizzy dat she jes' draps right down an' he gobbles 'er up in a jiff. Yassuh, he's a gre't schemer. He nuver do anything in a hurry, jes' plan hit all out good an' den teck his time to hit. He say he gwine git even wid Molly Hyar' yit if it teck 'im twel Chris'mus, an' 'twuz gittin' 'long to'des dat time befo' he wuz raidy fer' 'er.

"One col' mawnin' he went streakin' thu de woods, lif'in' up one paw an' stoppin' ter lissen fer de dogs now an' den, but de coas' wuz cle'r an' he kep' on 'twel he got ter Mis' Molly's. He knock on de do' but she ain' year 'im kase she wuz busy rockin' two er de chillen whar wuz sick, an' singin' at de top uv 'er voice ter drown de noise de urr chillen wuz makin' ez dey racket roun' de house, playin' hoss an' leap-frog an' ketcher, an' cuffin' an' tusslin' 'twel ol' man Hyar' wuz 'bleeged ter leave de ol' ooman de bag ter hol' an' teck his pipe an' go an' set on de bench outside de do' tẹr git some peace an' comfu't.

'Mis' Hyar' kep' on singin' at de top uv 'er voice:

'Oh Bunny is my baby,

Bunny is my lamb,

I lores my Bunny better

Dan a gre't big dish er ham.

'Oh Honey is my baby, Honey is my lamb,

I loves my Honey better

Dan a gre't big roas'ed yam.' 


\section{AT THE BIG HOUSE}

“' 'Prat dish yer Bumny an' Honey,' sez Fox ter hisse'f, sezee, 'wimmin-folks sut'n'y does mek fools er deyse'fs over dey chillen, an meks de chillen fools, inter de bargum.' Wid dat he fotched a big lick on de do' wid his walkin'-stick an' Mis' Molly gin a scream an' jumped so't she mos' drapt Bunny an' Honey. She ax

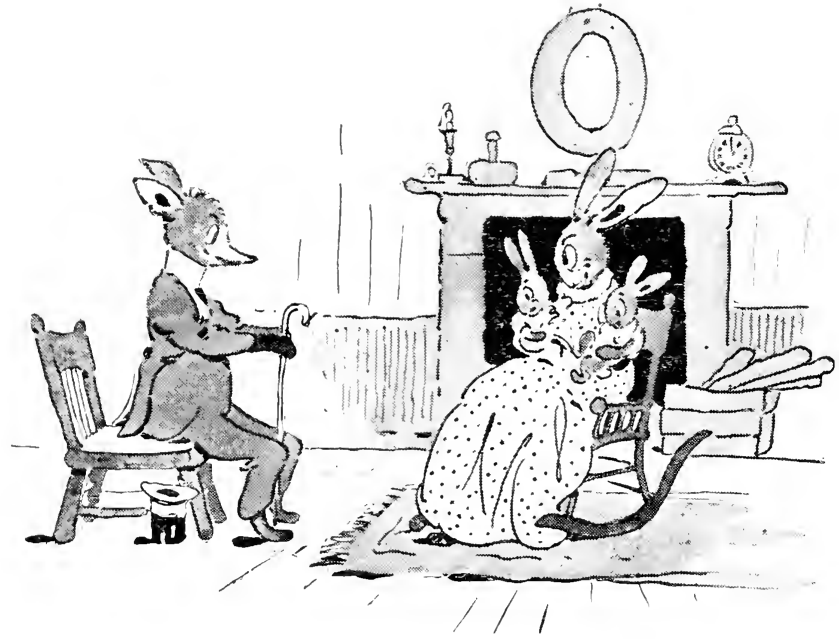

'im fer ter come in, an' she snt'n'y wuz s'prise w'en she see who 'twuz, but she ain' let on, not her; dat wan't her way. She mek 'er manners an' ax 'im fer ter git a cheer fer hisse'f, 'kase he cu'd see dat her han's wuz full, an' den she 'mence ter run on 'bout de wedder same 'z folks does dese days w'en dey ain' know w'at else ter say.

“Fox he wuz mighty p'lite an' mannerly an' chock 18 


\section{MR. FOX AND MOLLY HARE GO FISHING}

full er pooty talk. He say, "Clar" ter goodness, Mis" Molly Cotton-tail, you sut'n'y does look snipshus. 'Pears lak you git rounger an' younger er'y ear, you sut'n'y does, ma'am.'

"Molly snicker, but she wan't tucken in by him. She say, 'Hysh, man! Better not let Mis' Fox year you go on dat-a-way! 'Sidesen dat, I knows I ain' no mo' ter look at dese days dan a lean crow wid a graveyard cough.'

"Sho! Mis' Molly?' sezee. 'You ain' do ro'se"f jestice. 'deed you ain'. I nurer has see you lookin' better. I'll back you 'gins' all de triflin ' young gals roun' dese diggin's,' sezee. He run on dat-a-way ontwel he think he got her good an' please', an' den he say, 'Mis' Hyar', I done call roun' ter see ef you onn lak ter go fishin'. I knows a monst'ous fine place, whar de fishes is thicker'n blackba'ies in a patch, an' I teck you right dar ef you sesso. 'Tain' no fur ways, neener.'

"Mis' Hyar' she say, she do, 'Thanky, Mistah Slickry; thanky, suh. I wish ter gracious I could go wid you, but you see how 'tis. Yer's Bunny an' Honey sick on my han's; real croupyfied, dey is, an' de urr chillen cuttin' up lak de ol' Harry, an' all my wu'k layin' roun' loose. I 'clar' dem chillen gwine run me 'stracted. You Blinker! you Winker! come yer, bofe un you, an' set yo 'se fo down by de chimbly an' stop dat urerlas'in' scufflin'! Jumper an' Thumper, I wants you ter come yer an' shake han's wid Mistah Slickry Sly-fox an' do lak you had some raisin', stidder gwine on wid dat fistan'-skull-fight right in front er de comp'ny.'

"De chillen done lak she tol' 'em. an' Fox he kep' on 'suadin' an' 'suadin'. Mis' Molly mighty fond er gwine 


\section{AT TIIE BIG HOCSE}

romn' 'joyin' husse'f an' she ain' none too fond er housew'k, so las" she say. "Well, Mistah Sly-fox, I dumno how in de worl' I gwine wid rou, deed I don'. But molbe I kin git de ol man ter look atter de chillen. an’ of I leave plenty er pollygollic an' squilts an' hoarhoun'-an'-boneset tea fer Bumny an' Honey, I reckon dey ${ }^{*} l l$ git on, an' I kin set out a col' snack fer 'em all 'twel I git back.'

"So she call de ol' man ter come in an' min' de chillen, an' I tell you he come right slow, draggin' his footses an' knockin' de ashes outen his pipe. She show him de rittles an' gin him de pollygollic an' squilts an' de hoarhoun'-an'-boneset tea an' tol' him ter dose Bumny an' Honey ev'y time dey cried. Wid dat de chillen all set up a turrbl' squall, but she ain' pay no 'tention, jes' tucken her shawl an' a ol' baskit an' putt out fer de branch wid slickry Sly-fox.

"On de way she say, 'Mussy me, Mistah Slickry, w'at we gwine do fer poles an' lines? I wuz so boddered up, gittin' 'way f'um dem chillen, dat I ain' think nuttin' 'bout poles an' lines.'

“'Temmine,' sez de Fox, sezee, 'I done tuck kyare er dat. I kain't be pestered kyar'yin' poles an' lines back an' fo'th, so I keeps 'em hid 'way in a ol' holler tree nigh de branch. I'll fit you out all right, Mis' Molly; don you 'sturb yo 'se'f 'bout dat.'

"Dey went 'long mighty fren "ly an' familious, an' ol' Fox git so monst'ous p'lite dat las' he say, 'I 'clar' ter gracious, Mis' Molly. you mus' 'scuse me fer bein' so onmannerly ez ter let you kyar' dat baskit. 'Tain' fitten fer a lady lak you ter do dat. Please, ma'am, ter lemme tote de baskit.' 


\section{MR. FOX AND MOLLY HARE GO FISHING}

"Mis' Molly she gin 'im back jes' ez good 'z he sont. She say, "Deed, Mistah Fox, I kain't nohows think er lettin' a gemman lak you be seed totin' a baskit; you mus'n' name dat ter me no mo'.'

"Fox he 'sist an' sist, an' Molly she kep' on makin' out she ain' want 'im ter kyar' de baskit, but las' she han' hit orer to him, dough ef dey'd bin anything in hit, Mis' Molly 'd a-seed "im furder 'fo" she'd a-let 'im tote hit: she done know "im too well fer dat.

"Las' dey come ter de branch, an' Fox he putt de baskit on de groun' an' sot down on a log ter ketch his win' befo' he got ter wu'k. Mis' Molly she wuz honin' ter begin de fishin', so she say dat ef he'll jes' tell 'er whar de poles an' lines wuz hid, she'd go an' fotch 'em an' git de bait raidy.

"Fox he say, 'So do, Mis' Molly; so do. Jes' go up de branch yonner a li'l wars an' look in dat ol' holler sickymo' dar an' you'll fin' a lot er poles an' tackle, an' you kin jes' teck yo' ch'ice.'

"She went slitin" up de bank an' poked 'er haid inter de sickymo' tree, lookin' fer de poles. Bless goodness, 'twan't none dar! an' w'at's mo" tain' nuver bin none. She think mebbe she done gone ter de wrong tree, so she went traipsin' roun' ter ev'y sickrymo' she see on de bank an' git husse.f all frazzle out widout findin' nair' pole er line, w'ile ol' Fox sot up on de log smokin' an' laughin' ter hisse'f orer Mis' Molly an' de poles.

"Las' she come back an' tol' im dar wan't no sickymo' wid poles inside, an' he say, he do. 'W'at dat, Mis' Molly? You tell me you kain't fine dem poles? I'se 'bleeged ter see dat wid my own eves 'fo' I kin b'lieve hit,' an' wid dat he pull hisse'f up an' mosey ter de sick- 


\section{AT TIIE BIG IIOUSE}

rmo'. I'en he git dar he poke his haid inside an' den dror hit out an" squat down on his ha'nches an' drap his jaw open lak he'z so sprise he kain't talk. Las' he say, "Well, Mis' Molly! I'se dat flabbergasted I sca'cely kin git my bref. On 'y yist 'd'y I wuz yer, an' dem poles wuz all afe an' som!, an now some no-kyount, consumbunkshus thief-cr-de-worl' dome bin yer an' he'p hisse'f ter my propputty. I wish I had 'im yer dis minnit. I 'Gar' ter you l'd jes' natchelly wear dem poles out on his hide, dat I would! Seem lak a half-way decint pusson orter lef' me jes' one pole ter putt me in min' er de res'. I nuver knowed no pusson dat mean befo', lessen 'twuz de man whar shave hisse'f jes' befo' he die, so'ster cheat de barber outen de job. Yer you, Mis' MIolly, done come all dis way ter go fishin', an' nair' pole er tackle fer you. Hit sut'n'y is a shame.'

"Mis' Molly feel kind er saw'y fer 'im, so she say, 'Oh, nemmine, Nistah Slickry Sly. I ain' min' dat so much, but I is sort er disapp'inted not ter teck home some fish ter de chillens, 'kase I know dey all fixin' dey moufs fer a nice mess dis evenin'.

"Fox he study a w'ile an' den he say, 'Well, I tell you, Mis' Hyar', ef you be willin' ter fish lak I does, now an' den, mebbe we kin git a mess fer de chillen yit. Now you set up yer on de bank a minnit an' I show you dat ef you jes' got de gumption, you kin git de fish widou ${ }^{2}$ no tackle. Hits dat-a-way in de fishin' bizness, anyhows; you kin have all de pole an' line you wa ister, but ef you ain' got de gumption you ain' git de fish.'

"Wid dat he wag hisse'f down de bank an' stan' on de aidge wid his nose near de water, peerin' in. an' 'long come a fool young fish dat ain' know 'nuff ter know 


\section{MR. FOX AND MOLLY IIARE GO FISHING}

w'at 'twuz stannin' styarin' in at 'er. Ol' Fox ain' move a muscle twel de fish wuz right beneaf his nose. Den he splunge one paw in an' swipe de fish out an' lan' 'er in de baskit "fo" she kin flop "er fins twict.

"He call Mis" Molly down ter see, an' she wuz plumb tickelt ter def wid de way he done kotch dat fish. she say, Well, ef urer I see de beat er dat sence I bin chawin' rittles! Mistah Sly-fox, I'se 'bleeged fer ter git you ter l'arn me dat way er fishin'; hit beats de cl' way all holler.'

"Fox he say, "Tain' no trick 't all ter do dat. All you gotter do is ter putt yo' nose down in de water an' keep jes' ez still 'z dat rock yonner, an' de fus' fish you see beneaf yo nose, jes' swipe ro paw in an' git 'er.'

"Mis" Molly she do lak he tell 'er", an' stan' dar wid er nose poke down so'st hit tetched de water, fer ol' Fox ain let on to 'er dat he kep' his nose jes' outen de reach er de water. She stood dar an' she stood dar, but no fish ain' come "long, fer ol" Slickry he keep up a loud talkin' an' make all de rackit he kin so 'ter scare de fishes away. She stan' dar an' she stan' dar, an' hit git 'long to'des night an' turn colder an' colder, an' las' Mis' Molly git tired an' say she b'lieve she "bout raidy ter gin up an' go home. But Fox he aig "er on an' tell 'er not ter budge. "kase he sho" dat w'en de fishes come out ter git der supper dey boun ter come dat way. Water git colder an' colder all de time, an' Mis' Molly 'gun ter shake, an' her toofs chatter lak she have de ager. Las' she say, "Deed, Mistah sly-fox. I kain't stan' dis no longer; 'deed I kain't. I gotter quit dis minnit er drap yer in my tracks: dat I has.'

"Fox he chuckle ter hissef lak he know sump'n 23 


\section{AT TIE BIG HOUSE}

mighty funny, an' he say, sezee, 'Come 'long, den, Mis' Molly: 'tis mos' night. I reckon yo' chillen an' yo' ol' man be 'spectin' you long 'fo' dis.' Wid dat he pick up de baskit wid de fish in hit an' mek up de bank. Mis' Molly she wuz gwine foller, but, bless yo' soul, w'en she go ter lif' 'er haid, she foun' dat her nose done froze fas' ter de water, 'kase de ice bin makin' all de time she wuz stannin' dar, an' ol' Fox know dat mighty well w'en he call 'er down de bank. She pull an' she haul, an' she kick an' she thrash, but 'twan't no use; dar she wuz an' dar she stay.

"Fox he wuz up de bank jes' laughin' an' kyar'yin on. He git so full er laugh an' fun dat las' he tuck ter chasin' he own tail roun' an' roun' in a succle. jes' 'zackly de way de dogs does. Mis' Molly she year 'im gwine on, an' she sing out, 'Dat's all right! You got yo innin's fer onct in ro' life. High time you did done dat, seein how many times I done fool you, but I ses ter you dat you gwine langh on de wrong side er yo' mouf befo' I gits thu wid you, sho' ez my name's Molly Hyar" : dat you is !"

"She kep" on twis"in' an' turnin' an' tryin' ter wu'k 'er nose free, but 'twuz long time 'fo' she got hit a-loose, an' den she hatter leare a piece er de skin stickin' ter de ice. W'en Fox see she wuz onloose he stop chasin' his tail roun' an' pick up de baskit an' light out f'um dar widout stoppin' ter say far'-you-well, an' 'twuz long time 'fo' he had de insurance ter come whar Mis' Molly wuz.

" 'Tm-umph!' she say, ez she g'long home, 'dis sarve me right fer bein' sech a fool 'bout fishin'. Fishermens ain' got no sense, nohows. Seem lak de chanct er gittin' 


\section{MR. FOX AND MOLLY HARE GO FISHING}

one'r dem li'l scaly-backed creeturs jes' crowds ev'ything else clean outen der haids. Ter me, no fish, nose all skunt up, dish yer th'ee-mile walk befo' me an' a hongry ol man an' cross chillen at de een' ur hit. Atter dis I gwine stay home an' do de eatin' an' let some un else do de ketchin'?

"Wid dat she light out fer home, wid her nose jes' a-achin' her so's't she kain't nohow's hol' hit still. She keep a-twitchin' an' a-wu'kkin' hit ter git some ease, but seem lak she nuver git clean orer dat spe yunce, 'kase de hyar's been twitchin' der noses uver sence. Sometimes hit look lak dey's mekin' faces at you an' actin' sort er scawnful, hut 'tain' so ; hit's jes" "kase ol' Mis' Hyar' got her nose fros'ed dat time she went fishin' wid slickry sly-fox.

"An' now I gotter quit, fer I year yo' maw callin'. Ỵa'am, comin', jes' ez soon 'z I kin git dese chillen good an' tuck up in baid." 


\section{MR. IIARE IMITATES MIR. BEAR}

The next morning, after breakfast, the children heard a strange voice in the region of the back porch and went out to investigate. Aunt 'Phrony had come up to the honse to beg for quilt pieces, and as she sat on the porchsteps, waiting until her wants could be attended to, the children saw her for the first time. She was tall and thin and very straight, with high cheek-bones and piercing dark eyes. She wore a red handkerchief about her neck and large brass rings in her ears, and she claimed Indian blood, in proof of which she was in the habit of calling attention to her hair, saying "twan"t no nigger-wool." In fact, while it was closely kinked, its jetty tint and finer texture distinguished it from the coarse and rusty-black hair of the pure African type. When questioned as to her ancestry, she would say, if in a communicative mood: "Yas, Mars' Torm done buyed me and brung me up yer f'um Nawf Ca'liny. My daddy wuz a Nawf Ca'liny Injum, a Churrykee, an' my mammy she wuz a slare-ooman, dat huccome me ter be bawn a slave. Ef she'd a-bin a Injun an' him a slare, den I'd bin bawn free, 'kase de chillen alluz b'longed wid dey mammy; she free, dey free; she slave, dey slaves."

As Aunt 'Phrony sat with folded arms, gazing off at the rim of pines that formed a green horizon about the 


\section{IIR. IIARE IMITATES IIR. BEAR}

plantation, she looked rather forbidding, rery different from the fat and laughter-loring Aunt Nancy. The children did not renture near until she pretended to be suddenly aware of their presence and condescended to say. "Is dese Miss Janey"s chillen, whar I done year tell 'bout comin' down ter see dey Lncle Hinry? Come yer an' lemme see of air' one er you favers yo' maw. Huh-uh! Nair" one half ez good-lookin' ez der maw, er urer gwine be. But I dumno ez we kin 'spec' dat f'um chillen dese dars."

Totwithstanding this unfarorable rerdict, Aunt 'Phrony thawed by degrees, and presently Ned rentured to ask if she knew any tales. She shook her head. "Who? Me? Reckon I got sump'n else ter do sides studrin' 'bout tales. W'at kind er tales you talkin' 'bout?"

"Why, about the animals," explained Janey; "tales like those Aunt Nancy tells us."

At the bare mention of Nancy, Aunt 'Phrony pricked up her ears, for the two women were ancient and immemorial foes. Plantation gossip said that Aunt Nancy had coolly married out of hand the very man on whom Aunt 'Phrony had fixed her young affections, and had never been pardoned for doing so.

"Dellaws!" she sniffed, "dat ooman bin tellin' you-all tales? Mis'able ol' nigger tales, I be boun'. Ef I wan't gittin' so bad in my 'memb'ance an' so shawt in de bref I cu'd whu'l in an' tell you heap er Injun tales whar I useter year my daddy tell; dey beats all de nigger tales uver wuz knowed."

"Did any of the Indian stories have a rabbit in them ?" asked Ned. 


\section{AT THE BIG HOLSE}

"Rabbit?" said she. "You mean ol' Hyar"? Te ain" call 'im 'Rabhit' rer in Ferginny: jes' 'ol' Hyar'. er 'Mis' Molly Hyar'. er 'Mis' Molly Cotton-tail. De creetur go by all dem names. Dar wuz a Injun hyar' an' a nigger hyar', an' de Injun hyar' cu'd do mos" anything de nigger one cu'd, an' mo', too, inter de bargum."

"Aunt 'Phrony." said Janey, who was a little diplomat. "won"t you please tell us some of the things he did. so that we can see if he was anything like Aunt Nancy"s hare?"

'Phrony's pride was aroused. Determined not to be outdone by Nancy, she sat for a while thinking and then began:

"In de ol' times de Hyar' wuz de bes' known er de creeturs an' de bigges' man ur all, an' dis huccome so: he have de gif' er mummickin' anyb'dy an' ev'rb'dy, an' he go roun' 'mongs' de humans lettin' on he 'z dis pusson an' dat pusson an' tu'rr pusson, an' doin' all sorts er harm an" mekin' all kin's er mischief dat-a-way. Las" de people git tired er dis an' dey say dey ain' gwine stan' hit no longer, so dey git up a big hunt an' chase 'im clean outen dat kyountry. Den he go 'way off ter nu'rr place, whar dey ain' know 'im, an' set out ter bamboozle de crecturs same 'z he bin doin' wid de humans. mekin' out he 'z fus one thing an' den nu'rr. an' mummickin' er'ything he see folks do er year 'em say. Dar wuz one time w'en dem smarty ways come nigh bein' de def ur 'im. an' dat w'at I gwine tell you 'bout.

"One day he wuz gwine down de road, jiggitty-jig, wid one year turnt ter de back an" one ter de front, so's't he kain't miss yearin' ev'ything dat go on. w'en all ter onct he seed Mistah Growly Grum-b'ar comin', 


\section{MIR. HARE IMITATES IIR. BEAR}

lookin' mighty big an' empawtant. Hyar' ain' nuver met up wid 'im befo' an' he feel kind er jubous 'bout "im, so he serouch down by de side er de road in de hopes Mistah Bar ain" gwine notuss "im. But Bar he wall his eye rom” an” ketch sight ur “im, an’ he stop an` look down at "im outen de cornder ur his eye, lak Hyar" so small he sca"cely kin sce "im, an" den he say, "Souls an" bodies! who dis lil feller skulkin' yer by de side de road? Is I urer see you befo'? I kain't seem ter 'member hit, but den I done knowed so many ereeturs in my time dat you kain't 'spee' me ter weight down my min' wid 'em all. Co'se dey all 'members me; dey kain't he'p doin' dat, I reckon. but dey mus'n' git hu'ted in der feelin's ef I kain't krar' 'em all on my min'.'

"Hyar" boun" ter mek hiseef 'greeable of he kin, so he say, 'Naw, suh; I ain' nuver have de good luck ter meet up wid you befo', but ef I had, I en'dden 'spee' a sho'-'nuff gemman lak you is ter 'member no seeh trash ez w'at I is. I bin yearin' dis long time dat you is de bigges' man roun' dese parts, an' I has bin might ly sot on mekin' yo 'quaintance.'

"B'ar right please" wid dat sort er talkin' an' he ax Hyar' ter walk 'long wid 'im a w'iles so dey kin git 'quainted. Ol' Hyar' go sidlin' 'long wid 'im, tryin' ter teek big steps lak him, mighty 'feard dem big feet gwine tromple on 'im, but bowin' an' scrapin' an' sayin', 'Yassuh!" 'Jesso, suh!' 'I blieve you, suh!' ontwel Mistah Growly Grum think he's Big-man-me fer sho". An' I tell yall ehillen, nou, dat's de way ter git on wid folks. Jes' you sing small an' let dem kyar' de heft er de chume.

“Ol' man B'ar git mo' an' mo' empawtant an' g'long 


\section{AT TIIE BIG HOT'SE}

puffin’ out his chist an' layin' down de law wid one paw slapped on tu'r, ontwel dey git ter his house. He done mek up his min' by dat time dat Hyar" wuz de bes' comp ny he met up wid in a long time, so he 'vite him ter come in an' have some dinner. Hyar' say he ain'

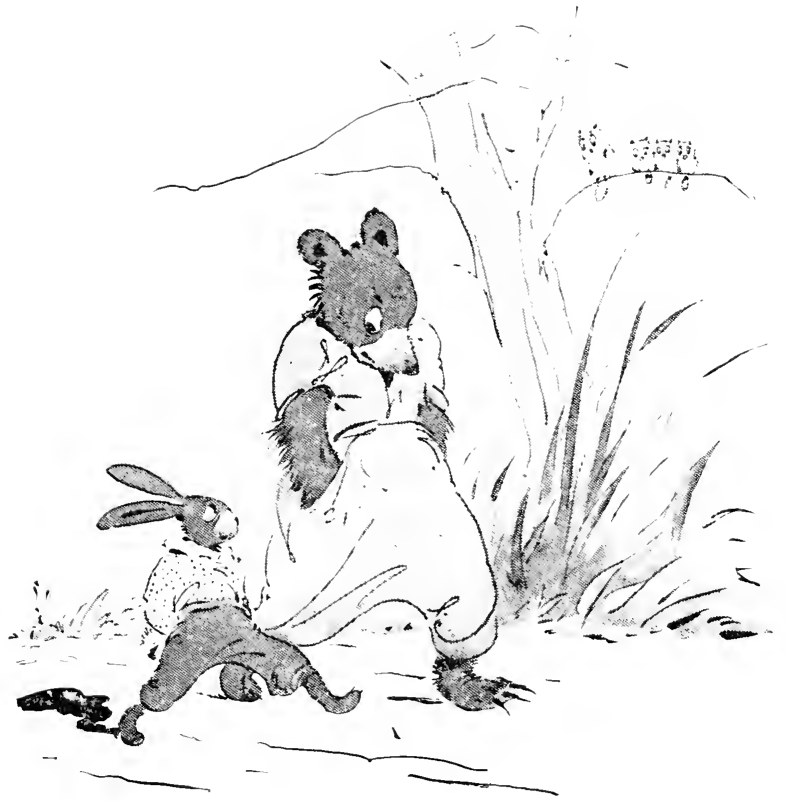

kyare ef he do, an' he go an' set down by de fire w'iles Mistah Growly Grum stir roun' 'mongs' de pots an' de kittles.

“All de time dinner wuz cookin' ol' Hyar' wuz jes' a-layin' hit onter Mistah B'ar wid his flattersome talk. 


\section{MR. HARE IMITATES MR. BEAR}

Sezee, "Well, you sut'n'y is a cook f'mm 'way back. I oon blieve sech a hig man ez wat you is cu'd be so handy ef I ain' see hit fer myecf.'

'Dar wuz a pot er peas on bilin', an' B'ar look roun' on all de shefs fer some fat ter putt in wid em, but 'twan't none dar. Den he tucken out a knife an' sharp hit up a li’l, an'ol' man IIrar's heart jump up in his mouf w'en he see dat. 'Name er gracious!' sezee ter hisse'f, 'lemme git outen dis! 'Pears ter me he gittin' raidy ter kyarve me up fer de dimner. Jes' my luck! Stidder gittin' smmp'n ter eat, I is gwineter git et myse'f.'

"But B'ar wan't studyin' 'bout him. He jes' walk up ter de pot an' cut a li'l gash in his neck an' let de grease run inter de peas. "Tain' hu't him 't all, 'kase de bars gits mighty fat in de fall off"n de mast whar draps foum de trees. Dey jes' stuffs deyse fs den, so't dey kin sleep all thu de winter widont wakin' up ter git sump'n ter eat; lives oft'n der own fat, dey does, all thu de col' wedder. Well, Bar's fat wuz so monst'ous thick jes' den dat he ain' feel de cut, an' g'long gittin' de dinner ez ef he ain' even got a scratch on 'im.

"Ol' Hyar' wuz might'ly please' an' clap his han's an' kick his heeks on de cheer-rungs, and mek gre't 'miration. He say 'twuz de handies' 'rangemint he urer see, ter kyar' yo' bacon rom' wid you in yo' own hide, an' he 'low ter hiscef, right den an' dar, dat he gwine do de same thing er bus'.

"Den dey drord up cheers an' sot down ter de table, an' Mistah Hyar' 'mence gwine on 'bout de dinner. Ile say, 'You mus' scuse me ef I ax fer nu'rr he p er dem peas. I lak ter look atter my manners, but 'deed yo' 


\section{AT THE BIG HOUSE}

cookin' done druy 'em clean outen my min'. Yo' fat got a flaror to hit dat suit my tas'e prezackly; 'deed hit have.'

"He run on dat-a-way, an' all de time he wuz jes' a-honin' ter try de same trick hisse'f, so las' he tell Mistah B'ar he gwine gin a dinin' nex' day an' he ax 'im will he come 'rom' an' he'p 'stroy vittles. He say he got a nice mess er peas he gwine treat him wid, but, bless yo' soul, de peas wuz right dat minnit in his naberfolkses gyardins.

'B'ar he say he come ef nuttin' hinner, an' Hyar' go kitin' back ter steal de peas fer de dinin'.

"Nex' day Hyar" go out ter meet Mistah B'ar an' bringed 'im in an' fotched a cheer an' gin 'im a pipe. He go cavawtin' roun' de room lak he's on springs, showin' off w'at a spry, handy man he wuz. He git de peas on bilin' an' den he look roun' in de cubberd fer de fat. Las' he say, 'Well, I'll be snickered! ef I ain' fergit de fat fer dem peas. Now, w'at I gwine do? Kain't gin you ol' po'-trash vittles widout no grease in 'em.' Wid dat he ups an' tecks a knife an' walks over ter de pot an' cuts a li'l gash in his neck. Lo, beholst you ! nair' smidgin' er grease drap out, but, mussy me! how de blood done spurt all over de ha'th, an' li'l mo' he'd bin a goner ef Mistah B'ar ain' bin dar ter bine him up an' doctor him atterwu'ds. B'ar wuz a right smart ur a doctor in dem days, fer he spen' so much time out in de laurel dat he know all 'bout yarbs an' sech ez dat.

"W'en Hyar' wuz on his footses ag'in, gwine roun' de house mighty limp an' low-down-in-de-valley, Mistah B'ar gin him a sho'-'nuff tongue-lashin'. Sezee, 'You 


\section{IIR. HARE IMITATES MIR. BEAR}

moughter knowed dey wan't no grease in a mis'able lean creetur lak you is. Yer $I$ is, all fat up fer de winter; dat de time w'en I gotter lay roun' an snooge an' have no chanct ter pick up vittles. But you is on de go, keepin' de paf hot f"um 'ear's een' ter 'ear's een. I lak ter know how you spec's ter lay up fat? W'at mek you think, anyhows. dat you kin do de same ez me, you po' li'l knee-high-to-a-hoppah-grass? Tm-umph! wat sort er worl' dish rer be ef all mens kin do de same things? You bes' g'long now an' 'tend ter yo' own bizness. an' set dis down in ro' 'memb'ance. dat dar ain' no pusson mek mo' mistakes er git mo' laugh at dan de man whar tries ter be tu'rr folks stidder bein' hisse'f. You year me talkin',', 


\section{TIIE FRIENDSHIP OF MIR. BEAR AND MIS' TLRKEY}

"Tell," said Ned, when Aunt 'Phrony had finished the story of how the hare imitated the bear, "I always thought that bears were cross and ate people up. I should think the hare would have been afraid to have him doctor him. I rouldn't want any old bear to come fooling around me."

"Who 'feard? Hyar'? Him! Naw, suh!" said Aunt 'Phrony, "he ain' knowin' w'at 'tis ter be 'feard er anything in dis 'rarsil worl'. Sidesen dat, I done tol' you he sof'-sawder ol' Mistah B'ar an' gin 'im 'nuff sweet talk ter fill a honey-gum. But Hyar' he wuz mo' diff"nt f'um tu'rr creeturs. "Twan't safe fer de res' un 'em ter fool wid Mistah Bar; 'deed hit wan't. Dat putt me in min' er de time w'en Mis' Tukkey think she gwine mek fren's wid 'im, an' dis how hit happen:

"B'ar useter live in de low-groun's an' wu'k fer his livin' same 'z tu'rr folks, but atter w'iles he git tired er dat, an' sezee, 'I jes' 'bout b'lieve I girine quit wu'k an' go whar I kin git me 'nuff ter do me widout usin' so much elber-grease in de gittin'. W'at de use er livin', anyhows, ef you gotter spen' all yo' time dustin' 'roun' atter sump'n jes" ter keep de bref er life in you? Naw, suh! 'tain' wuf de w'ile. lessen you kin do a lot er restin' up an' have some fun inter de bargum.'

"So he g'long off up inter de big mountains an' hide 


\section{MR. BEAR AND MIS' TLRKEY}

hisse'f erway in de laurel an' res' an' sleep jes' w'en he feel lak hit. an" de baluns er de time go romantin' up an' down de mountains kind er show an lazy, sort er studyin" whar he gwine putt his foot down nex, 'joyin' hissef might ly, widout doin" no w'k 't all, jes' pickin' up sump'n ter eat ez he trabel "long, an" dat wan't hart. He 'uz 'tickler fond er 'lases an' sweet apples, kase he got a mighty sweet toof, but honey wuz de dish w'at hit "im in de bull:-eye an go ter de right spot er'y time. He wuz willin" ter trabel miles ter fin" "im some honey, but ef he kain't fin' any. den de nex" bes" thing wuz a mess er raid ants. Hed sleep all day long on er ridge in de wom summer dars. but ef nu re bar come dat-away an" fetched "im a li"l tap in his sleep. he'd rar up on his behime laigs an" den dey'd have hit. fer all de worl' lak dese yer prize-fighters, boxin' an 'cuffin', an' wres"lin' 'twel one'r dem wuz th'own. Den tu'rr un 'ud grab 'im by de th'oat an' growl an' growl 'twel you'd 'a thought he 'z gwine eat 'im up. but pres'n' $y$ he'd onclinch an' let 'im go wid one las' growl ter tell 'im, 'You better not fool wid me erg"in. suh.'

"He live 'long dat-a-way, eatin' an' sleepin', wid a li'l walkin' an' fightin' th'owed in, 'twel one day he met up wid a li'l ol fool wil' tukkey. She was mincin' an' tippin' 'long, fer all de worl' lak dat no-kyount gal er Aunt Nancy's w'en she git on her sunday clo'es.

"Pres"n'y she see Mistah B'ar, an" stidder bein' 'feard, lak she orter bin ef she had a grain er sense in dat li'l ol' haid er her'n, she 'mence ter mek gre't 'miration. He look so big an strong she think he mus' be a monst'ous fine man, an' dar whar she git fooled, lak lots er wimmins I bin knowin'. 


\section{AT THE BIG IIOLSE}

"She hop up in front er him an" say, "Scuse me, Mistah Growly Grum, sulh, ef I axes you ter stop a minnit an' lemme look at you. Lan' er de livin”! I nuver is see a man tall ez wat you is. An' how big an' strong you looks an dat long brown fur you wears on yo' hide sho" is harnsum. Please, suh, lemme walk 'long wid you li"l ways an" look at you some mo"?'

"B'ar kind er growl sump"n nu'rr way down in his th'oat an' she tucken hit fer yes an' go tippin' an' mincin' 'long mo' wusser dan befo", mighty proud ter let tu'rr folks on de mountain see er in sech comp ny. $\mathrm{IV}^{*}$ en dey git ter Mistah B'ar"s house, she stick her haid in de do" an " w'en she see all de dirt an" de mess, for he wuz too mawtal lazy urer ter clean up de house, she say, she do, 'Oh, me! oh, my! Mistah Growly Grumbar, you sut'n'y does need a ooman ter look atter you an' cook yo' vittles an' tidy up de house. Please, suh, ter lemme stay an' wu'k fer you, an' de on'ies' thing I ax is de priv'lidge er settin' an' lookin' at you w'en I isn' busy.'

"B'ar say ter hisse'f, 'Dish yer a sho'-'nuff bawn fool, but mebbe she kin wait on me, so I reckon I let her stay, fer a w ille, anyhows.'

"So he say to "er. "Well, Mis' Tukkey, sence you is so pow"ful sot on hit. I spose you kin stay; but you mus"n' look at me too often, 'kase I ain' use ter bein' styar'd at an' I dunno w'at I mought do ef I got riled.'

"Mis" Tukkey say she do "er lookin' w'iles he'z ersleep, so he tell er ter stay ef she wanter.

"Den she whu'l in an' clean up de house, an" cook 'im a good dinner, an' w'en he tucken a nap she git 'er a 


\section{MR. BEAR AND MIS' TLRKEY}

li'l bush an kieep de flies offen him, an' set an' look an' look ez ef she ain' nurer gwine see 'im erg"in.

"She kep" hit up dat-a-way fer so long time, an' git wusser an wusser 'bout him er'y day ontwel at las' she dunno how in de worl she gwine keep f"um lookin' at "im w"iles he wuz wake.

"Now Bar he had de fashion er gwineter sleep er" $y$ day "bout de time he mence ter git a li"l hongry, an' so he wuz mighty ap" ter dream 'bout sump'n nu'rr ter eat, an watsomurer he dream bout, dat de thing he 'bleeged ter have w'en he wake up, an' he alluz g*longed out an got hit.

"Mis" Tukkey fin" dis out, so er'y day w'en he wake up she step to'des 'im an' say, 'Mistah Growly Grum, Mistah Growly Grum, wat you done dream bout dis time? Please fer to tell me, suh, so I kin know wat fixin's ter git raidy ter go wid hit fer dinner.'

"B'ar he 'ud tell her, an' den go 'way an' git w'atsomurer hit mought be.

"Mis" Tukkey git so sot-up on kyount er 'sociatin" wid Mistah Bar dat tu'rr creeturs culd scacely putt up wid her foolishness. but w'en dey talk hit orer dey alluz tell one nu'rr dat sump'n bleeged fer ter happen ter teck down her fedders.

"I done tol" you she git mo'n mo' sot on Mistah B'ar. an' kep' squintin' at 'im 'roun' de cornders an' cuttin' her eye at 'im w'en he wan't lookin' ontwel de wunner is dat he ain' ketch 'er long befo'. But his eyes wuz set so clost toge rr. same ez wid all de bars, dat hit mek him near-sighted. an' ef he see you a li'l piece off he ain' kin tell you f'um a stump. But Mis" Tukkey 


\section{AT THE BIG HOT'SE}

git so owdacious dat atter w"iles he do ketch 'er, an' he soon git tired ur her an her foolishness. He ain' sayin' nuttin', "kase he wan't knowin' jes" w'at he gwine do 'bout hit. Las', one day, he see her lookin' at him jes' "zhe wuz wakin" up f"um his nap.

"she walk up ter him, same 'z alluz, an' say, 'Mistah B'ar, Mistah B'ar, wat you done dream 'bout dis day?' An dat mimnit hit come inter his haid dat he gwine fix er den an' dar.

"He strotch hisse'f a li"l lak he wan't quite thu wid his nap an' den he eye 'er up an' down an' he say, 'Mis' Tukkey,' sezee, 'you is growed tol'ble plump an' fat, I see, sence you bin stayin' in my house an' quit yo' runnin' up an' down de mountains.'

"Mis' Tukkey kind er snigger an' look down, 'kase she mighty please' ter have him notuss 'er. 'Yassuh,' sez she, 'I b'lieve I is growed mo' plumper dan w'at I wuz.'

"'Yas, ma'am! you sho'ly has fat up,' sezee, 'an' now I ax you dis,' sezee, 'ain' I tucken you in my house an' fed you an' gin you shelter, an' ain' you promuss dat you oon look at me 'seusin' w'en I wuz sleepin', an' isn' you done bruk yo" wad?"

"Mis' Tukkey she stamnin' on one foot lookin' mighty oneasy, an' she say, she do: 'Deed, Mistah B'ar, you mus' 'scuse me dis time, 'deed you mus', 'kase I kain't he'p hit, 'deed I kain't.'

"Den he riz up f'um whar he wuz settin' an' he say, way down deep in his th'oat jes' ez growly ez he kin, 'You mus' scuse me, ton, Mis' Tukkey; I kain't he'p mysef, 'deed I kain't. You ax me w'at is I dream, I tells you I done dream "tukkey," an' you knowin' me. 


\section{IIR. BEAR AND IIIS' TLRKEY}

well 'nuff ter know dat w'en I dream tukkey I 'bleeged ter eat tukkey, an' wid dat he fell 'pun 'er an' she scuffle some an' de fedters flew'd 'poun' a li'l an' den er'ything mighty quiet, kase she done gone whar she cu'd see de inside er Mistah B'ar stidder de outside. De crecturs whar she bin puttin' on airs wid say hit sarve 'er right fer tryin' ter leep comp'ny wid 'er betters."

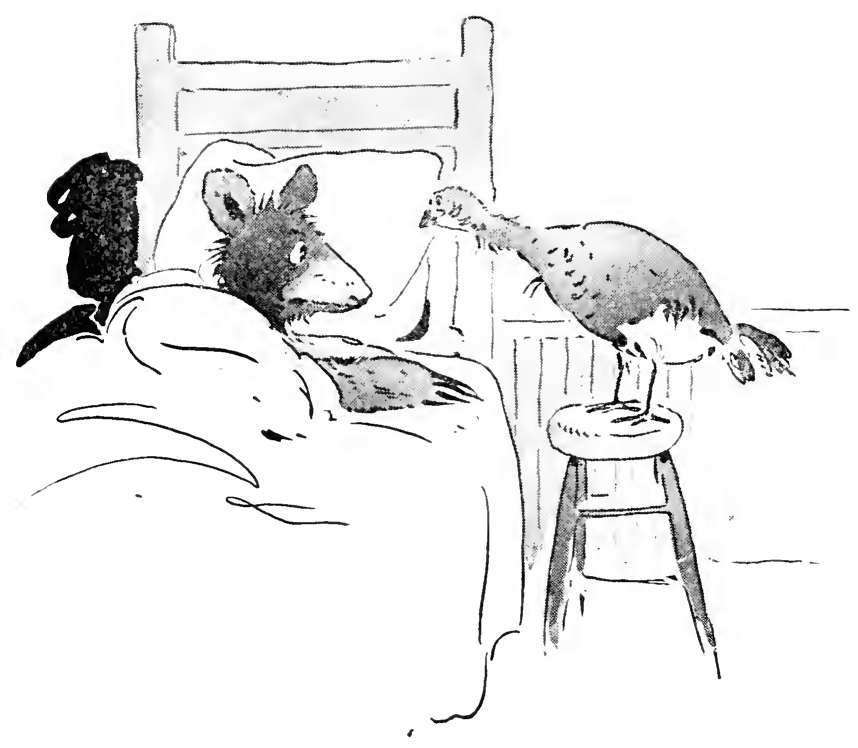

"I think that was a real mean old bear to eat her up after she waited on him and did so much for him, don t you, Aunt 'Phrony?" said Janey.

"Laws-a-mussy, chil," said Aunt 'Phrony, who had had unfortunate matrimonial experiences, "w"en you is ol' ez w'at I is, mebbe you'll fin" out dat ef a man wants 


\section{AT THE BIG IIOLSE}

ter git shed ur a ooman. he ain' gwine think 'bout w'at she been doin' fer 'im, he jes' fergit ev'ything 'ceptin' dat he do wanter git shed ur er, dat's 'nuff fer him, an' he don' let dat outen his min' ontwel he done finish de bizness.

"'seem ter me I year yo' maw callin' me, an' I reckon I hes" mosey 'long upstairs ef I 'spec's ter git me any quilt-pieces dis day." 


\section{THE TOAD, THE GRASSHOPPER AND THE ROOSTER}

Aunt Nancy lived in a little cabin on the edge of the - woods, not far from the "big house." The children were not long in finding her whereabouts and in making the acquaintance of her granddaughter. Cass, an orergrown girl of seventeen, who was just beginning to have what Aunt Nancy called "fool notions" about dress and beaux; and also that of her dog, Bouncer, a lean, hungry and disreputable hound by whom she set much store on account of his pedigree and "p ints." Just what his raluable points were, no one but Xancy had ever been able to discorer, though many a neighbor whose erening meal had been snatched in a twinkling beneath his rery eyes could bear witness to points which were considered distinctly undesirable.

The little cabin was scrupulously clean within and held many things which the children had never seen before-the gay patch-work quilts on the beds. the strings of red peppers hanging from the rafters, the curious old salt-gourd, polished and dark as mahogany from years of service. The path and dooryard were swept clean, and at one side was a little garden in which flourished homely old-fashioned flowers. marigold, zinnias, larkspurs, princess' feather. cockscomb and hollyhocks, hardy and gay, needing no coaxing to 


\section{AT THE BIG HOLSE}

cheer these humble folk with their coarse, bright beauty. Next to Bouncer in Aunt Nancy's affections were her precious "posies": Cassy had to content herself with third place. The little people fell into the habit of paying frequent risits to this cozy spot, always finding a warm welcome from Aunt Nancy and often getting the treat of a story besides. The first time they visited her she celebrated the occasion by relating certain doings of the toad, the grasshopper and the rooster.

"I reckon dar wan't no mo" livelier creeturs in de ol' times," she began, "dan w'at dem th"ee wuz. Mis' Hoppah-grass, w"icht some un "em called her 'Mis' Popeves,' 'long er her bulgy eves, she wuz a gre't darnser, darnse roun' in de grass all day long, an' dar wuz some talk er harin' 'er up befo' de meetin' 'longer her triflin' ways. Toad-frog he spen' all his time gwine huntin' atter bugs an' sech, an' de way he git roun' over de groun' wuz a caution ter snakes. He ain' stop ter walk, jes' natchully lipt up f'um one place an' went ker-swish! thu de air an' lit on de nex" place. I let you know he wuz a soople man in dem dars, dat he wuz. He wuz a curisome sort er creetur, anywars. Jes' lak de toad-frogs dese days, he had two little bags er white juice right back ur his eyes, an' ef anything got atter 'im er tried ter ketch 'im, he jes' spurted dat juice right out on 'em an' dey wuz glad ter let him 'lone. You kain't 'suade no dog ter tackle a toad, he ain' wanter git dat p ison sprinkelt on "im. Toad-frog he wuz p'tickler 'bout his dress, too; git 'im a new suit er clo'es er'y now an' den, jes' lak de toad-frogs does now. Fus' dey 'mence splittin' up de back an' den dey know hits time ter pull off de ol' clo'es, w'icht dey skins 'em down orer der legs jes' lak 


\section{THE TOAD AND THE ROOSTER}

Nars' Ted do his li'l trousers, an' dey pulls an' hauls, usin' der moufs ter he'p pull, an' w'en de ol' elo'es is off dey rolls 'em up in a bunnel an' jes' natehully swollers 'em, dat dey does; I done seed 'em wid my own eres.

"Chicken-roostah he wuz a monstous fine puffawmer wid his roice: he ain" do much but practuss hit, an" he wuz dat proud he kain't wait fer de daylight, but git up early in the mawnin' an' wake tu'rr folks long 'fo' day singin':

$$
\begin{aligned}
& \text { 'Cock-a-doodle-deedle-doo, } \\
& \text { I hin sing mo' loud dan you, } \\
& \text { Cock-a-deedle-doodle-dee, } \\
& \text { Git up an' lissen, folls, ter me!' }
\end{aligned}
$$

"I ain' hatter tell you dat folks wan't likin' 'im any too well. 'kase he alluz 'mence his singin' jes' 'bout de time w'en dey wants ter turn over an' have nu'rr li'l smooge. Sidesen dat, dey think he'z a kin' ur a ol' hypermocrit, "kase he sa'nter 'long wid Mis" Hen an' de chillens, 'tendin' lak he 'z he pin' scrateh fer de livin', an' de on'ies thing he do wuz ter light in, w'en Mis' Hen gin a cackle ter say she done foun' a bug er a wu'm, an' snatch hit way f'um 'er 'fo' she kin say Jack Robumsum.

"Atter w"iles dey git tu'rr ereeturs down on 'em, 'long er all der singin' an' darnsin' an' no-kyount, shif"less ways, an" w'en dey see dat, dey think mebbe hit time ter turn orer a new leaf an' whu'l in an' 'arn der livin'. So dey git toge'rr an' have a confab an' mek up der min's dat dey gwine run a farm in cahoots. 


\section{AT TIIE BIG HOUSE}

Roostah he wuz 'p'inted ter be de plow-han', dat 'kase he have sech strong claws jes' fitten fer ter scratch up de groun' wid: 'Toad-frog he wuz ter be de hoe-han', an' Nis" Hoppah-grass she wuz ter stay home an' do de cookin' an' look atter de house.

"Dey 'vide de vidjun er de wu'k dis-a-way fer so long time, an' dey git on mighty well an' wuz might'ly please', 'kase de new wan't rub off yit, but pres'n'y Mis' IIoppah-grass she git kind er tired an' lazy, fer she ain' nurer do a lick er work befo' in all her bawn days. She drap de skillit an' fall back in a cheer an' putt her footses on a stool an' tie her haid up in a hank'cher an say she feelin' so ailified dat she know she in fer a spell er sickness, but dey neenter sen' fer de doctor, 'kase she sho' she gwine die, anyhows, an' she wanter teck her own time to hit an' not be hurried inter de nex' worl' by all dat truck he mek folks swoller. She mought ' $z$ well die easy, she say. She talk 'bout de doctor plumb scannelous, 'kase she ain' want him ter come dar an' let out de news dat de on'ies thing w'at ail her wuz laziness.

"Den she clim' up on de baid an' laid dar groanin' an' kyar'yin on, an' de men-folks stan' roun' an' look at 'er a w'ile, sort er he'pless, an' den dey ses, 'Well, I reckon dar ain' nuttin' we-all kin do,' an' dey g'long off ter wu'k. Roostah he wuz plowin' in a fur fiel' an' Toad-frog he wuz hoein' near de house, so dey 'gree dat he better git dinner. Dat huccome he hatter do de hoein' an' de cookin' an' tidy up de house, let 'lone waitin' on Mis' Hoppah-grass; she fin' sump'n fer 'im ter do ev'y five minnits endurin' er de day.

“Dey kep' hit up dat-a-way ontwel he wuz wo'n ter 44 


\section{THE TOAD AND THE ROOSTER}

a frazzle, an' he say he 'bleeged ter have sump'n er ru'rr ter churr him up an' drive de tire away. 'Bout dat time he camed 'cross de rim ur a ol' meal-sifter an' dat putt a notion in his min'. He tucken de rim an' strotch a piece er sheepskin orer hit, an' den he got him a piece er fence-rail an' whittle hit down an' fasten hit on de rim. Pres'n'y he fin' a ol' cow-hawn an' he wu'k dat up inter pegs, an' den he git him some catgut strings an' strotch 'em 'cross de sifter. Den he screw 'em up wid de cow-hawn pegs 'twel he git 'em in chune an' las' he swipe one han' crost de strings, an' suz! she 'mence ter talk, an' 'twuz a sho'-'nuff banjer! one'r dem reg'ler ol' plinketty-plunketty nigger banjers whar got mo' git-up-an'-git to 'em, w'en it come to de marter er foot-shakin', dan any er dese yer shiny, primp-up banjers whar hangs in de sto' winders an' tries ter git folks ter walk in an' buy 'em. Nigger know better'n dat!

"W'en Mistah Toad-frog git de banjer chune up jes' 'zackly ter suit 'im, he set outside de do' ev'y day w'ile de dinner cookin' an' pick dese yer darnsin' chunes whar mek eren chu'ch members feel lak dey jes' natchully 'bleeged ter git up an' knock time wid der footses. One day w'ile he wuz pickin', he think he year a soun' er darnsin' on de flo' inside. 'Day er grace!' sezee, 'is my years done trick me, er is dat darnsin'? Kain't be Mis' Hoppah-grass, 'kase she too po'ly. Hit 'bleeged ter be, dough, fer she's de on'ies pusson in dar.' He stop playin', darnsin' stop; he go on, darnsin' go on. He putt his year down an' lissen at de banjer ter see ef anything rattle,-banjer she wu'kkin' all right, den he know 'twuz Mis' Hoppah-grass. He ain' let on, an' he 


\section{AT TIIE BIG IIOTSE}

wait on "er jes' de same, but w"en Chickin-roostah come home, Toat-frog tucken him off a li'l ways an' he say, 'Mistah Roostah, wen you comes ter dinner to-morrer, don' you git up on de fence an' crow lak you bin doin' ter lemme know hit"s time ter dish up de vittles: stidder dat, you creep up sof ly w iles I pick, an peek in thu de do'?

" "Trell, befo" de king!" sez de Roostah, sezee, "wa t's in de win' now, Mistah Toad-frog?"

“ 'Temmine, sez de Toad-frog, 'you g.long an' do lak I tells you, an' I boun' you see a sight fer sore eyes.'

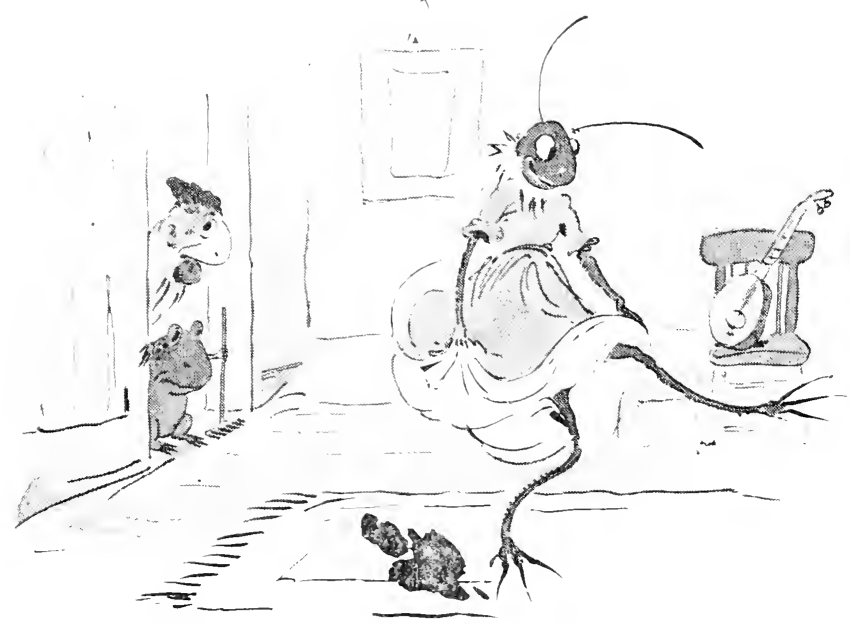

"So de nex' day w'en Roostalh come home ter dinner, stidder crowin' ter say he be lar soon, he crope up ter de do' an' peeked in thu de crack w'ile Toad-frog pick de banjer 'twel she plumb talk, an' wish't I mought die? 


\section{THE TOAD AND THE ROOSTER}

ef dar wan't Mis' Ioppah-grass darnsin' rom' de room jes' ez well 'ez she uver wuz. She caper an' she twis' an' she turn an' she do de back-step an' cut de pigeon-wing an' wound up wid de double-shuffle. Las' ur all she lipt up an' cracked her heels toge 'rr an' spun clean rom' in de air befo' she lit on her footses.

"Roostah ain' sayin' nuttin', he jes' beckon ter Toadfrog. He nurer stop pickin', but tiptoed ter de do" an" cut one eve thu de crack, an' got dar in time ter see "er do de high-jump. Dey wuz dat outdone dey kain't say nuttin', jes' hunch one nu'rr wid der ellsers. Den Toadfrog fell back on de bench an' went on pickin', an' Roostah he go an' git up on de bench an' crow lak he alluz do jes' befo' dimner.

"W'en she year "im crow, Mis" Hoppah-grass lipt back in baid an' drord up de kivers an' done lak she ain' kin move han' er foot. Den Roostah walk in an' come up to'des de baid an' he say, sezee, "Wellum, how you come on dis mawnin'?

"She wuz pantin" so f"um de darmsin' dat she sca"cely kin git her bref. so she roll her eyes up in her haid an' say, 'So po'ly, sca'ce kin talk.'

"Dat 'ar"s a burnin' fib!" sezee. 'I boun' you I mek you git up f"um dat an walk, Mis' Pop-eres!' sezee.

"She jump outen de baid an" mek fer de do", an" Roostah he lit out atter her, an' dey sut'n'y had it den, fer she wuz a sho"'nuff jumper an he wuz one"r dese yer shawt-legged mens whar do lak dey gwine fall all over deyse'fs w'en dey runs fas'. Dey went starin' thu de rard an' orer de fence an' 'crost a fiel an' den back ergin. an' he nigh mos' chase "er down de well. Den she mek fer de grass an' think she gwine hide 'way 


\section{AT TIIE BIG IIOLSE}

f'um him dar, but he putt on a big bus' er speed an' kotched an et her jes" ez she wuz stippin' inter de grass.

"I reckon y’all done seed de chickin-roostahs chasin' de hoppah-grases befo" now, an" dish yer I been tellin' you is de reason dey does so. Dey ain' fergit her 'ceitfulness, an'seem lak der jes" natchully kain't leave a hoppah-grass 'lone w'en dey sees er, boun' ter run her down er die." 


\section{MR. TERRIPIN GETS THE NOSE-BLEED}

"Now, Aunt Nancy," said Janey, when the old woman had finished her story about the deceitful grasshopper, "we just aren't going until you tell us another story."

"Hi yi! w'at kind er talk is dis I year outen de mouf ur a li'l white lady!" exclaimed Aunt Nancy, who, while secretly flattered at having her little friends linger, was such a stickler for politeness that she could not let a breach of it pass. Tery zealons indeed were the old mammies about forming the manners of the children under their charge, and rery high the standard of politeness which they felt should belong to the "quality." So she went on: "Is dat a pooty way ter ax fer nu'rr story? Sidesen dat, is I said anything 'bout yo' goin' home? Naw, suh. an' I oon say nuttin' ef yall set dar 'twel de day er judgmen'. I don' treat my comp'ny data-way. I hopes I know my manners better'n ter ax my comp'ny ter go home, lak a li'l gal ax de Thompson chillen tu "rr day w'en she git huffed wid 'em."

Cassy had been watching the pots and pans simmering on the hearth in preparation for the modest dinner, closely watched in turn by the greedy Bonncer, who lay close to the hearth with his nose in his paws, apparently asleep, but with one eye on the dimner. Every time she passed. Cassy administered a little furtive punishment, at which Bouncer each time raised up the voice of complaint. 


\section{AT THE BIG IIOUSE}

"Look yer, gal," Aunt Naney finally inquired, "w'at ails dat dog er mine?" Cassy answered innocently, "'Deed I dunno, granny, he 'pears ter be mighty oneasy, startin' an' rappin' dat-a-way in his sleep."

"Tell, rou come outside yer, wid we-all, an' I boun' you my dog git "long all right," commanded Aunt Nancy.

"Law, granny," said the girl, "you know mighty well dat ef I leare Bouncer in dar wid de dinner he gwine git hisse'f outside ur hit in thee shakes ur a sheep's tail."

"Well, den," said the old woman, "jes' you bring de dog out yer wid you whar I kin keep my eye on bofe un you; I ain' gwine have my dog cuffed by no pusson, lessen I sees fit ter do hit myse'f."

With that, Cassy and the reluctant Bouncer came out and joined the little circle about the doorstep, Bouncer on the extreme edge, watching his chance to steal into the honse unperceived. They effected a welcome diversion, for Janey was feeling rather guilty orer her incivility to the Thompson children. When Cassy and Bouncer had subsided, Aunt Nancy resumed her story telling, relating the adventures of Molly Cotton-tail and Mr. Terrapin while on a hunting expedition.

"Hit happen dis-a-way." she began. "One time Mis" Molly Cotton-tail an' Mistah Tarr'pin wuz tol"ble fren 'ly fer a right smart uv a spell. an' w'ile things wuz gwine on lak dat, Mis' Molly she met up wid him one day an' she say: 'Mistah Tarr'pin, I dunno how 'tis wid you, suh, but I is sort er tired er de vittles I bin harin', jes' dese yer peas an' cabbage an' gyardin'truck in gin'l, 'long wid a li'l grass. I knows you bin 


\section{MR. TERRAPIN GETS THE NOSE-BLEED}

havin' de same sort er stuff an' seem ter me you looks a li'l pindlin'. Ef we cu'd jes' go huntin' an' git ow'se'fs a li'l fraish meat, I boun' you we'd pick up an feel sprucy right off.'

"Tarr"pin ain' bin notussin' dat he felt bad, but w'en Miss Molly talk dat-a-way he felt sort er doncey an' painyfied dat mimnit, an' he say he spec' 'twould do him good ter have a li'l meat-vittles, so wid dat dey up an' went huntin' toge 'rr. Dey go up an' down de woods, perusin' roun' fer w'at dey kin fin', an' w'iles dey sa'nter 'long Mis' IIyar' she wuz talkin' an' argyfyin' wid Tarr 'pin 'bout dis an' dat, tryin' her bes' ter aggervex him, 'kase she wuz chock full er talk an' mighty please' w'en she kin fin' anybody ter 'spute wid her. She ain' git much sassifaction f'um Mistah Tarr'pin, 'kase he wan't no gre't shakes at talkin' an' he lak ter do ev'ything slow an' easy, 'twuz come-day, go-day wid him.

"Mis' Molly so tucken up wid her own talk dat she ain' notuss w'at gwine on, an' dat de reason she ain' see Mis' Panter comin'. She bin rampagin' up an' down de woods huntin' fer sump'n ter eat, an' 'bout de time she git so hungry she sca'cely kin stan' hit, she seed 'em comin'. 'IYyar' ur my whiskers!' sez she, 'dis whar I gwine whu'l in an' git me my dinner. Two kin's er meat ter onct, lawsy, lawsy, w'at luck I is havin'? She see dey ain' kotch sight ur 'er yit, so she mek has'e ter' strotch husse'f 'cross de road an' 'tend lak she daid.

"Molly Cotton-tail so busy talkin' dat she ain' see Mis' Panter yit, but w'en dey git ter whar she wuz layin' 'cross de road, Mis' Molly say: 'Jumpin' Jehoshaphat! ef we ain' got de luck dis day, Mistah Tarr'pin, 


\section{AT THE BIG HOUSE}

den you kin call me a sinner. Yer meat all raidy ter ow" han 's, an" fraish kilt, too, ef I ain" mek no mistake, 'kase she wo'm yit.'

"Den Tarr'pin he say, sezee: 'I 'spec' you is right, Mis' Molly. you mos' in gin'ly is. But dish yer wat troublin' me: I kaint see how we gwine tote dis meat home, 'kase look lak she monst'ous hairy.'

"Mis' IIyar" she putt her haid on one side, an' cock one year up an' tu'rr down an' look fus" at Panter an' den at Tarr'pin, lak she wuz medjin' 'em bofe. Las' she 'low, she do, 'Mistah Tarry-long Tarr'pin, you know I is a soon ooman an' a willin ooman, but I ain' got much strenk in de back-bone. Tow you got de hardes' back-bone an' de bigges' back-bone fer yo' size ur any man whar I knows an' 'pears ter me dat ef I kin jes' git her laid 'cross you an' git you started, mebbe we kin mek out ter tote her home.'

"Tarr'pin 'gree ter dis, so he run his haid unner Mis' Panter an' Mis' Molly gin her a shove, an' dar she wuz. Den ol' Tarr'pin more off, an' ef he wuz a slow man befo' I dumno w'at you call him now, slower dan 'lasses in Jinnawary. Mis' Hyar' mek out dat she wuz shovin' behime, but stidder dat she mount up on de kyarkiss an' po' ol' Tarr'pin drug her "long, too. 'Bout dat time he say, 'Mussy me! Mris' Molly, seem lak dis meat done got heap hairier all ter onct!"

“'In co'se, Mistah Tarr'pin,' sez de Hyar', sez she, 'in co'se dat 'bleeged ter be de way. fer de kyarkiss gittin' mo' colder all de time, an' de daider she is de haivier she boun' ter be.'

"'I reckon dasso,' sez de Tarr'pin, sezee, an' he g'long pantin' an' puffin', 'but I let you know', sezee, 


\section{MR. TERRAPIN GETS THE NOSE-BLEED}

'dish yer de bigges' contrac' I is urer mnertooken, an' hit's de fus' an' de las' er de kin'. I let you know dat.'

"All de time dey 'z gwine 'long. Mis" Panter she jes" retch down one claw an' scratch, scratch, scratch mighty sof'ly on ol 'Tarrpin's neck. so't he sca'cely feel hit, an' pres'n'y de blood 'gun ter drip. drip, drip. Atter w'ile Tarrpin he seed de blood drippin' an' he 'spose 'twuz a fit er de nose-bleed. so he call out. 'Hit' Mis' Molly, I done tote so hard I'se tucken wid de nosebleed, an" I be "bleeged ter stop at de branch an" sta "nch de bleedin “' sezee.

"All right,' sings out Mis" Hyar', an' she clim' down f'um de krarkiss an' come roun' ter de front an' done lak she bin pushin hard all de way an' wuz plumb wo' out. She he"p onload an" den she squat down by Panter w"ile Tarrpin go ter de branch ter wash de blood off. Ol' Tarr'pin fin' 'twan't no nose-bleed; stidder dat sump'n done cut his neck mos' off. 'Bout dat time, w'en he 'z feelin' mighty weak an' skeery, Mis' Panter she gin a spring at him an she think she done got him dat time, 'kase he ' $z$ too weak ter git 'way f'um her. But sho! ol' man Tarrpin wan't bawn rist'd'y. He jes' gin a roll an' over he went inter de water, an' den he wuz safe, fer Mis' Panter wuz lak all de res' er de cats, she don' go in de water ef she kin he'p hit, she ain' got no use fer gittin' her paws wet. W'en Mis' Panter see Tarr'pin wuz clean gone she say "Well, I gotter have my dinner, come w'at may. go w'at may; so I 'spose I hatter putt up wid dat mis'able lean' li'l Mis' Molly Hyar", mighty po' pickins she is, I be boun?'

"W'en Mis' Hyar' see de kyarkis git up an' spring atter Tarr'pin, she wuz dat sprise' her eyes fair jump 


\section{AT TIIE BIG IIOLSE}

outen her haid an' growed big an' roun' ez saucers, an' she jes squat dar on te bank mon half palyze ontwel the sed Mis' Panter done turnt her 'tention on her. 'Im-umph! gimme leg-bail outen dis!' sez she ter husse'f, an' she mek tracks fer a ol' holler tree nigh de hank. She wuz a mighty spry ooman, but dis wuz one time w'en hit come nigh bein' good-by-Mis'-Molly-Cotton-tail, kase Mis" Panter wuz so clost onter her dat wen she run inter de tree she nab her by de tail an' bit hit plumb off. In dat huccome you see all her fambly gwine roun urer sence den wid sech a misable li'l tuff er white hyar in de place ur a tail. I reckon you done year follis call 'em cotton-tails 'fo' now, but I don' spose you is urer year de ol' song whar run on 'bout de creeturs' tails, sump'n lak dis:

\section{Raccoon's tail got rings all roun', 'Possum's tail go bare, Ol' IIyar' got no tail at all, Nuttin' but a bunch er hya'r.}

"Yex" time y'all meet up wid a ol" Tarr'pin, ef you tek de trouble ter look you kin see dat he's made in a mighty curisome way 'bout de neck, an' dis tale I jes' tol' you 'splains de wharfo:. Dat li'l ridge all roun' is whar de blood git crusted atter Mis' Panter saw mos' thu. 'Lrer sence den his haid look lak hit jes' kind er serunched down in his neek an' mought drap off ef you shuk him right hard."

“An' didn't Mis' Panter ever get any good dinner?" asked little Kit, eridently sorry for the hungry Madam Panther. 


\section{IR. TERRIPIX GETS THE NOSE-BI.EED}

"Now lissen at de baby!." cried Aunt Tancy. "Bless yo" soul, honey. I reckon you kin let Mis' Panter alone fer fillin husse fup, sooncr er later. She wuz dat kin' ur a creetur; she ain' gwine stop 'twel she do fin' sump'n nu'rr. But dis wuz de time $w^{\circ}$ 'n she ain" git Mistah 'Tarr"pin ner Mis' Hyar' neener, 'susen de een' ur her tail."

Just then there was a thud inside the cabin, which Aunt Tancy and Cassy both seemed to associate with the absence of Bouncer. Who had taken adrantage of the absorbing climax of the story to steal in unperceived. They hastencd in, only to see Bouncer hurriedly disappear through the back done with a ham-bone in his mouth. Aunt Nancy was full of wrath, but it was all directed toward Cassy instead of the dog. "Look-a-yer, gal," said she, "ain' I tol' you 'fo' now 'bout houn' dogs: You know how dey is: dey kaint he p de way dey"s made. You orter be "shame er yo se fer temp' de po" dog dat-a-way wid de dinner. Anybody whar leave good vittles settin' on de hath orter know wat she gotter 'spec'. I give you good warnin', gal, atter dis you gotter keep de dinner outen de reach er dat dog, er I gwine know de reason w'y." 


\section{HOW THE 'POSSUM'S TAIL BECAME BARE}

The children, in roaming through the woods one day, came in sight of the little clearing where Aunt 'Phrony's cabin stood, so hidden in the midst of tall pines that its existence was unsuspected until you were fairly on it. The old woman was in her tobacco-patch, stooping down and looking the plants orer carefully, leaf by leaf, in search of insects.

The children ran toward her as fast as their little legs could carry them, crying: "Oh! there's Aunt 'Phrony! there's Aunt 'Phrony! Please, Aunt Phrony, stop and tell us a story."

She went on with her work. looking up at the children sidewise. now and then, and muttering as if half to herself: "Dunno no mo' tales, dese chillen done drug 'em all outen me long befo' now. Sidesen dat, s'pose I stops my wuk, who gwine git dis bacey? Wy, de bugs an' de wu'ms, dat who. An' w'en de winter done come an' I wanster set "long side de fire wid my pipe, whar I gwine git me any baccy? Kin y'all tell me dat?"

"Buy it." suggested .Taney: "there's lots and lots of stores in town where they don't sell anything el:e."

Aunt 'Phrony raised her hands and rolled her eyes. "Fer de lan' sake!" said she. "does y'all think I made er money? Ain' I knowin" dat dar's plenty sto"s an' 


\section{HOW THE 'POSSEM'S TAIL BECAME BARE}

plenty 'baccy. but de money ain'so plenty, I kin tell you. Whar you reckon I git de money?"

"Out of my bank." piped up little Kit. nodding his head so fast that the rellow curls bobbed and danced on his shoulders. "out of my "ittle bank. I'se dot "leventy-fire coppers in it. an I'll dir em all to Aunt 'Phrony when the naughty bugs and worms eat up her baccy. "Es I will!"

The old woman's heart was softened by the generosity of the little bor, so she stopped work and gave him a hug, saying: "Bles de baby! now jes" fer dat I gwine gin him a story all fer hise fe, dat I is. Ef you thaves yo'se'fs, de res er you kin liwen. too, I reckon, but I wants yall ter know dat dis tale b'longs ter de li'l boy whar settin right yer by me.

"I nigh mos" think I gwine tell 'bout ol' 'Possum an' his doin's, 'kase he's de man whar seem ter riz up in front er my min' dis day. Ef y'all ain' seed him, y'ain' knowin' dat he got a tail mos' ez long ez w'at he is, an' dat bare dat Mistah Rat hatter git up early in de mawnin' ter show de ekal ur hit. But in de day an' time whar I tells you bout, hit wan't dat-a-way. His tail wuz jes' ez big an bushy an' harnsum ez de one ol' Mistah Slickry Sly-fox have sech a time hidin' 'way f'um de houn's an' de hunters. An' ef he wan't proud er dat tail, den I dumno proud w'en I see hit. Nair' fine day dat ol' Mistah Poky 'Possum ain' go perawderin' thu de woods wid dat tail hilt high up in de air fer tu'rr creeturs ter look at an' mek 'miration over. He g'long switchin' hisse'f sump'n lak Miss Janey do w'en she git on dat new raid dress. De creeturs dey stood hit fer so long time, an' den dey call a meetin' 


\section{AT THE BIG HOUSE}

ter talk de bizness orer an' see w'at dey gwine do 'bout hit. 'kase dey wuz all er one min', dat sump'n 'bleeged ter be done.

"Mistah Creechy Cricket he wuz dar, tryin' ter keep a still tongue in his haid long "nuff ter year w"at tu' folks wuz sayin', but "twuz mighty hard wu'k. "kase he wuz one'r dese yer talky-talky mens whar wanster keep der chins goin' all de time. He nurer let his ol' ooman slip in a wu'd f'um one 'ear's een' ter tu'rr: you ain' nuver year a chirp yit outen Mis' Cricket. Ex'y now an' den endurin' er de meetin' he let out a chirp, an' dar whar he putt his foot in hit, fer w'en tu'rr creeturs yearn him, dat gin 'em a notion, an' dey ses ter one nu'rr, 'Hi! he de one ter git atter ol 'Possum an' gin 'im a sho "'nuff tongue-lashin' an' tell 'im jes' prezackly w'at we-all thinks ur 'im.'

"So dey name hit ter Cricket, but he shake his haid an' say, 'Huh-uh, gemmen, I lak mighty well ter 'commodate you 'bout dis, but me an' Mistah 'Possum done fall out some ser"l 'ears back, an" sence den I ain' bin speakin' wid 'im-w"en I kin he"p hit." Right dar tu'rr creeturs nudge one nu'rr an" wipe off a grin on der coat-sleeves, for dey knowed cricket jes" blecged ter let out a chirp now an' den, 'kase he got one'r dese yer tongues whar hung in de middle an" wags at bofe een's.

"Sho! sho!" dey ses. 'Mistah Creechy, is you sho''nuff mad wid 'im?' He say, he do, 'Dat I is! an' you say good reason w'y w'en I tells you 'bout hit. I wuz hoppin' 'long one day, quiet an' peace'ble. not sayin' nuttin' ter no pusson, w'en I seed 'im come prancin' down de road, wavin' his tail an' actin' mighty airish. Dat sight kind er rile me. an' mebbe I sniffed an' let 


\section{HOW THE 'POSSUM'S TAIL BECAME BARE}

out a chirp er two an' ses "um-umph!" I ain' so sho' bout dat. But howsomurer dat mought be, he stop me an' ses. "Mistah Creechy Cricket, I done bin wantin' a talk wid you dis long time. I gotter tell you, fer de good er de hull passel er creeturs, dat yo' voice ain' true, you is mo' times offen de key dan on hit, an' me an' all de res' be might "ly 'bleeged of you goes off som'ers by yo ‘e'f ter do dat nerlas"in’ practussin' whar keeps folks foum gittin dey natchel slumbers. I ain' git a wink er sleep dese fo' nights han' rumnin'." I ses ter you, gemmen, dat I wuz plumb riled by dat, 'kase not long befo' I spen' de night practussin' my voice nigh de holler tree whar ol man 'Possum sleep, an' I 'clar' ter you, I done year him snore fum de dark ontwel de daylight, an' wat's mo' he snore so loud dat I kain't year my own voice an' ain' knowin' whe'rr I sing $O l^{\prime}$ IIunderd er S"archin' Praises. let 'lone keepin' on de key. I up an' tol' him 'bout de snorin', but he ain' b’lieve me an' jes' laugh in my face an' walk off, fer I tell y'all hit heap mo' easier ter mek a man think black is white dan ter mek him blieve he urer snored a snore; he alluz gwine putt de blame on some urr pusson.'

"Cricket git so mad 'bout den dat he kain't say nuttin' fer a minnit, an' de creeturs crowd roun' him an' aig him on. 'Well, Mistah Cricket,' dey ses, 'he sho' did gin you imp'ence! Dat 'nuff ter aggervex you! Now de time fer you ter git even wid de sassy trash, an' we-all gwine back you up.'

"Dey git him so w'k up dat las' he say he do de job, but he oon tell 'em how, 'ceptin' dat he ain' gwine have no wu'ds wid 'im. Den he hurry way f'um de 


\section{A'T THE BIG HOT'SE}

meetin', 'kase he know of he star long 'nuff he 'bleeged ter tell somebily wat he girine do.

"Dat night he crope up ter de holler tree whar 'Possum wuz sleepin" an" went ter wu"k on dat tail er his"n. He tucken one hya "r at a time an" gnor hit mos" thu. an' dey wuz so many in de tail dat twuz a all-night job, an' ef he ain' bin sech a soon man he oon git thu den. He talk 'way ter hisse'f all de time. 'Possum sho' would 'a yearn him, 'ceptin' dat he snore all night widout eren stoppin' ter turn orer. Yit w'en ol' man Coon call nex" mawnin' ter ax him howdy, he say he feelin' mighty po "ly an' ain sleep nair" a wink endurin' er de night. I done bin year humans talk lak dat befo" now, an' dey's mighty tetchy ef you tells 'em dey slep' mo`n dey think fer. Dat's one'r de times an' casions w'en you kin fool yo'se'f mo' easier dan not, 'kase co'se w'en youse 'sleep you dumno nuttin' 't all 'bout hit.

"W"en de sun git good an' up, Mistahi 'Possum go out, same'z he bin doin', fer ter show hisse'f off befo' de face an' eves er de creeturs. He wus prancin' 'long, monst'ous biggitty, wid his tail hilt high in de air, w'en he git ter a damp. swampish place whar kind er gin him de sniffles. He fotch a big sneege whar shuk him up all over, an' I 'clar' ter you dat w'en he git thu ev'y single hya'r done drap offen his tail an lef" hit bare an' smoore ez de p’am er yo" li"l han'.

"He ain' knowin" muttin' 't all 'bout dat. dough, an' g'long airish ez urer twel he come ter whar Mistah Cricket an' a passel er de creeturs wuz layin' low fer him ter pass, "kase Cricket done tol' "em 'bcut w'at he done, an' dey knowed de hya'rs wan't gwine stick on dar long. 


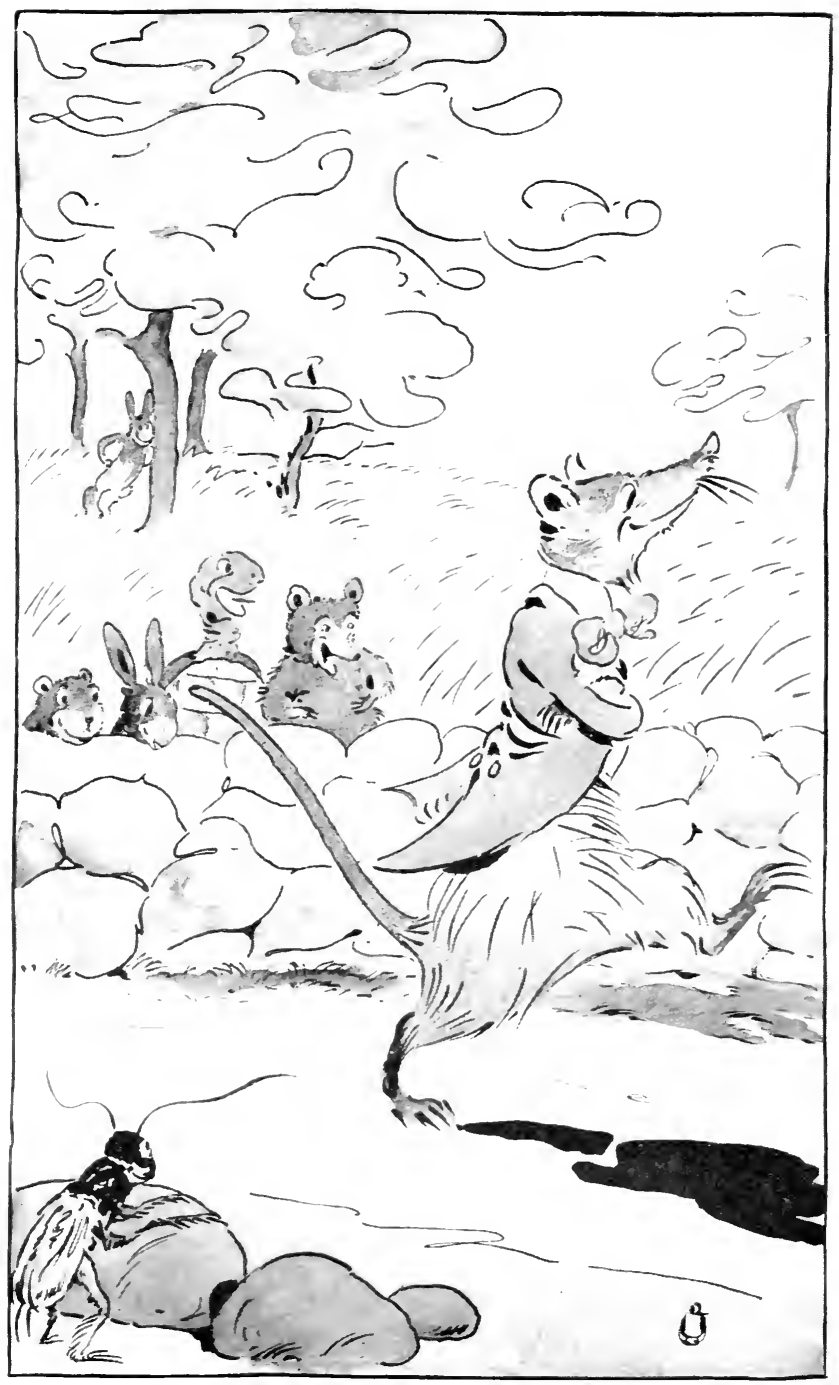

DEY SEED 'POSSLY SWITCIIN' DOWX IDE ROAD 



\section{HOW THE 'POSSEM'S TAIL BECAME BARE}

"When dey seed 'Possum switchin' down de road, showin' off dat ol' bare tail lak "twuz gre"t shakes. an' he not knowin' any diff"ns, dey bus" out laughin" so hard dat some un 'em 'bleeged ter lean up 'gin de trees, an' some un 'em git so sore dey hatter hol' on ter der sides ter ketch bref. Cricket so pleas' dat he kain't hol' his tongue, an' he chirp up loud 'z he kin,

\section{'I done done hit, I done done hit, Nair' a hya'r is lof' umm hit.'}

"Possum ain' knowin' w'at C'ricket mean by dat, but tu'rr creeturs shet him up, an' den dey smoove down der grins an' wait for de ol foller ter come up, jes' kind er snortin' an' snickerin' a li'l behime one han'ez dey say, 'Heyo, Mistah Poky 'Possum. whar dat tail er yo'n? W'at dat 'ar you drag behime? 'Clar' ef dat don' beat my time! You sho' is lef' yo' tail at home, you sho' is! Yo' tail nuver look dat-a-way: mus' b’long ter Mistah Rat! Lan' er gracious! 'Possum done bin ter de barber an' got him a clean shave dis time!'

"Las' 'Possum look behime, an' gre't-day-in-demawnin'? ef t'wan't nuttin' dar but jes' de ghos' ur his ol' tail. He felt so 'shame' ur hit an' ur de way he bin showin' off dat he drap down right den an' dar an' let on lak he wuz daid. An' sence dat time, w'ensomurer he see folks comin' he git ter thinkin' 'bout dat bare tail an' 'memb'in' how fine hit useter be an' how biggity he useter ac' 'bout hit, an' he feel so mosified over hit all dat he alluz drap down an' roll over an' tend dat de bref er life done gone outen him. Heap er times he fool folks whar ain' knowin' him. 


\section{AT TIIE BIG HOT'SE}

"Dish yer tale splains how "tis 'Possum come ter change his ways, an' stidder sleepin' in de night-time he sleep all day an" walk all night so "s't his bare tail kain`t be seed. Folks whar hunt him gotter go by night an' kyar" torches wid 'em so"t dey kin th'ow a light on him and let him know dey sees dat ol' bare tail.

"Ir"en de creeturs seed him layin' dar lookin' lak he sho "'nuff daid. dey felt kind er saw'y fer him, an' dey go rom" sayin' ter one nu'rr dat dey reckon he wan't sech a monst'ous fool atter all. But Cricket he mighty sharp an' up-ter-snuff. an' he kind er have his spicions er 'Possum, so he stay hidin' roun' w'en de res' go 'way, an' bimeby he see 'im git up an teck nu'rr look at de tail an' go slinkin' off in de bresh. Den Cricket jump up on a high bush an sing at de top er his roice:

\section{'I done done hit, I done done hit, Nair' a hya'r is lef" upun hit.'}

"An' he so might"ly please" wid w'at he done dat he bin keepin' up dat song uver sence. an' you kin year him at hit all night long, lessen you sleep ez soun' an' snore ez loud ez w'at ol' man 'Possum useter." 


\section{WHY THE 'POSSLM IIAS BLACK EARS}

The children were becoming proficient in the art of obtaining stories, and Janey in particular har early discovered that a succesful method of getting another was to ask questions about the last one, so she made haste to siy :

"Aunt Phrony, after the Posmm': tail became bare were the animals friendly with him, and did they like him any better :"

"Law bless you, honey," she said, "he wuz a change" man, he wuz dat. He ain have nuttin" lef" ter give hisse'f de highfalutins 'bout. an he mighty 'shame' ur his foolishness, so he go roun' grinnin' at er'y pusson he meet an' tryin to curry faror wid er ybjy, an' he bin doin' dat urer sence. I boun' you he d grin at youall ef he met up wid you. Tu'rr creeturs git so dey kind er lak him, an' dey ses ter one nu'rr tat he sut'n'y look ter be a pleasan' sort er pusson. Sidesen dat. he ain' have nuttin' lef' fer 'em ter be enviable 'bout, an' hit heap mo' easier ter lak folks whar ain got nuttin' you wants an' ain' seem ter be no mo' empawtant dan w'at you is.

"So dey treat 'Possum kind er decent, an' las" him an" Mistah Tarry-long Tarrpin git ter be mighty thick. Hit wuz 'long to'des de fall er de ear. jes' atter fros", w'en de 'simmons is ripe. an' ol' man 'Posum wuz lookin' right plump an' fat, 'kase he bin gawjin' hisse f 


\section{AT TIIE BIG IIOCSE}

wid 'simmons. 'Posums is pow'ful fond uv 'em, an' dat de reason. I reckon, dat some folkis calls em "possumapples" stidter "simmons. But whatsumurer name you calls em by. I knows dey mek mighty good simmon beer." Here she began humming as if to herself,

\section{"Sezee ter hisse"f. "Wid right good hiyare} I'll mek tre gallons er' simmon beer." "

Ned, who was rery fond of music, begged Aunt 'Phrony to give them the whole song, which she finally did, protesting, however, that " 'twan't nuttin" but one"r lese rer ol'time nonsense songs, nohow," and that it had nothing to do with the 'Possum, but was all about a lazy person whose habits cost him his lady-lore. It was called

\section{THE YOUNG MAN WHO WOLLDN'T MAKE CORN}

Grine sing a li'l song, an' 'tain' re'y long, All 'bout a young man dat oon mek cawn;

De reason w'y I nurer cu'd tell.

'Tase dis young man wuz alluz well.

IIe go ter his furm an' he peep in, Grass an' de hog-ueeds up ter his chin, Grass an" de hog-ureeds growed so high Dey mek dis young mun fer ter sigh.

Goes ter his neares' naber's house,

Goes a-co'tin', ez ue-all s'pose;

De gal u'uz a peart un, sho'z yo' bann.

Sez she, 'Young man, has you hoed yo' caun?' 


\section{WHY TIIE 'POSST M HAS BLACT E.LR}

'Oh, no, no, no.' u'uz his reply,

'I think hit's time I laid hit by,'

But befor he plant. hit unz July,

An' in september he laid hit by.

In October dar come a white fros',

An' de seed ur caun dis young man los';

Sez de gal. 'I'se single. an' so witl remain,

Fer a lazy man I won't maintain.'

One fruit tree on his farm dar grew, An' ex"y 'enr bore some good fruit. too;

sezee ter hisse'f. "Wid right good hiyare

I'll mek two gallons er 'simmon beer.'

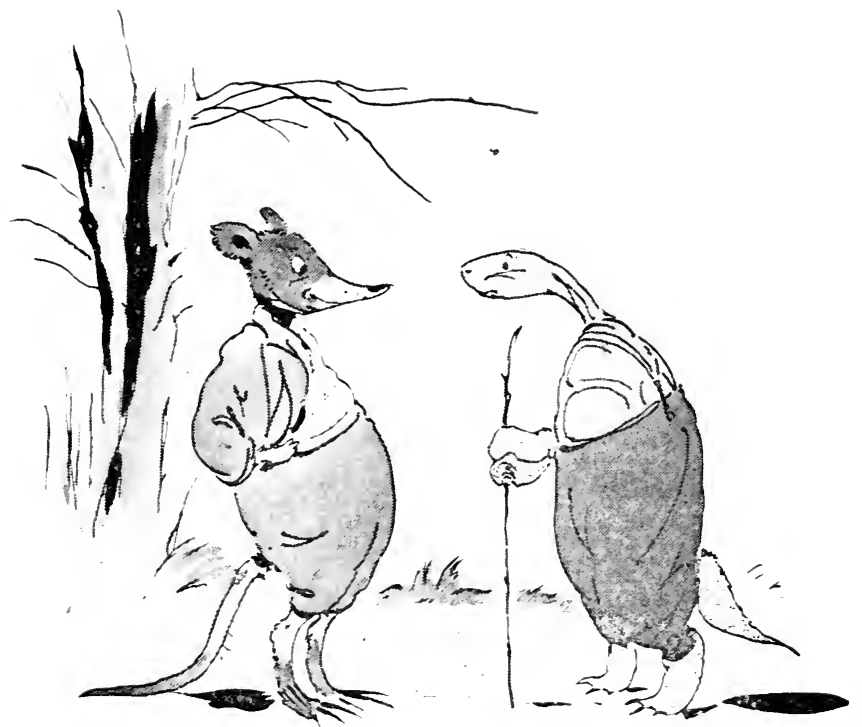




\section{AT TIIE BIG HOLSE}

"But nemmine de beer, lemme git back ter de tale. IV'en Tarrpin see how fat 'Possum look, he say, 'See rer. Brer Poky 'l'oswum. seem ter me you lookin' right plump an pealt dese days, an' yer me, so po' dat you kin see er'y bone in my body; jes' feel me if y'ain' blieve me. I is a plumb skillitin. I wish you tell me, sulh, w'at you bin doin' ter fat up dat-a-way.'

"Den 'Possum grin' an' he say, sezee, 'Dat my sekert, but you is my fren' an' I don' min' tellin' you. Well, suh, I gits dis-a-way in de fall 'long er de 'simmons. 'Simmons is ripe den, an' dar ain' nuttin' ekal to 'em fer puttin' de kiverin' on a man's bones. I sut'n'y is saw'y ter see you look so swivel up an'pindlin', an' ef you kin keep a still tongue in yo haid, I tek you out one 'r dese nights an' git you a mess er "simmons. 'Twon't do ter let tu'rr creeturs know, 'kase dey oon be 'nuff ter go roun'; mebbe you has notuss dat dar ain' no mo' sca'cer tree dan de 'simmon tree.'

"Tarr"pin say: 'I sut'n'y is s'prise. I bin thinkin' simmons wan't wuff shucks fer eatin', 'kase onct I tucken one in my mouf, an' suz! she drord so dat my mouf swunk up 'twel I wuz 'feard I wan't nuver gwine be able ter tek in a good moufful er vittles ag'in.'

"'Possum laugh fit ter kill at de idee er Mistah Tarr'pin eatin' green 'simmons. Sezee: “'Tain' er'y pusson got de sense ter tek things at de right time; seem lak mos' un 'em mus' grab right in, an' dar whar dey come out missin'. I reckon dat how 'simmons come ter have sech a bad name wid mos' folks. But de fros' done bin yer now. Brer Tarry-long, an' 'simmons is prime, an' ef you won't tell, I tek you 'long wid me dis re'y night.' 


\section{WIY TIIE 'POSSTM IIAS BLACK EARS}

"Ol' Tarr"pin he say, 'I cross my heart. suh, tat I won't tell, 'deed an' 'deed, an' doulle 'deed.' So dat night 'Possum tucken him thu de woods ter a tall tree whar wuz jes' ez full er 'simmons ez she kin stick. 'Now, den, Mistah Tarr'pin,' sezee, 'you gwine stay down yer on de groun' an' I gwine climb de tree an' shake fer you.' He went a-kitin' up de tree, swingin' f'um one limb ter tu'rr by dat long tail er his'n, whar he cuid wrop roun' things heap easier sence de hya's all fell off, "twel he fair" mek ol' Tarr"pin's haid swim. He shake an' he shake an' he shake, an' de 'simmons fall an' dey fall an' dey fall, an' all de time he shuk he w'uz singin':

"Possum's up de 'simmon tree, Go 'Tong, Josey;

Oh, please, Wistah'Possum, tor shake fer me, Go 'long, Josey, go 'long.

De tree mighty tall, an' de limb mighty bare, Go'long, Josey;

Tek kiyare, Mistah 'Possum, tek kyare, tek kiyare, Go 'long, Josey, go 'long.

I boun' you. ol' 'Possum guine tek' good hiyare.

Go 'long, Josey:

Dough de tree mighty tall, an' de limb mighty bare,

Go 'long, Josey, go 'long.

He wrop his tail all roun' de limb,

Go 'long, Josey:

You hin shake de 'simmons, but you lain't shake him, Go 'long, Josey, go 'long.' 


\section{AT TIIE BIG HOUSE}

"Possum wu'k on so busy lat he done fergit ev'ything else, an pres'n he git ter shakin' harder an' harder an' singin' louder an' louder. 'Bout den Mistah Wolf come lopin' long thu de woods an yeard him. 'Now, w'at kind er outlan'ish gwines on is dis. in de daid er night?' sezee ter hisse'f; 'T'se 'bleeged ter turn outen my way an' see 'bout dis.'

"W"en he git ter de tree he see po' ol' Tarr'pin stuffin' hisse'f, an' he say, sezee, 'Mistah Tarr"pin, w'at sort er lay-out is you got dar?' Tarr'pin say, he do, 'Oh, dish yer jes' a li'I medincin I'se tekin' 'kase I'se kind er run down an' po"ly.'

"Wolf he low hit look ter him ez of some er dat medincin ad 'gree wid him might'ly. Sezee. 'I think hit nigh 'bont jes" suit my case,' an' wid dat he wade plumb inter de 'simmons an' et 'em up so fas' dar wan't none lef' fer Mistah Tarr'pin, 'kase he nurer hurry bout anything, not even de puttin' 'way uv his vittles.

"'Possum up in de tree sced w'at gwine on below, an' sezee ter hisse'f. 'Now dis de time an' 'casion w'en I gwine fix Mistah Wolf so's't he kain't gobble up urr folkses 'simmons fer one w'ile, mis'able ol' big mouf!'

"He tuck an' tuck a rock he kyar"d wid him, an' he wrop his tail good an' tight 'roun" a limb, an' he lean outen de tree so 'ster tek good aim, an' he drap hit so close ter ol' Wolf dat li'l mo' she'd 'a hit him on de nose. Wolf year her fall ker-chug! an' she thump so haivy he think hit mus' be mighty big 'simmon, an' he 'low ter hisse'f dat he gotter have dat un ter fill up de las' chink ur his stummick. He 'z dat greedy he ain' stop ter bite ner tas'e ner chew; he jes' swoller her hull, an' stidder gwine down, dar she stuck; he kain't gulp her 


\section{WHY THE 'POSSLA HAS BLACK EARS}

down ner cough her up; dar she wuz an dar the stay. IIe roll, he waller. he kick. he cough, he try ter all fer he"p, but "tain no nee, an I let you know "twan't long 'fo" he stop kickin" an" strotch hise'f out an" jes' natchelly die fer de want er bref.

"Den 'Posmm he skunt down de tree in a hurry an' zamine" de kyarkiss ter see of he'z sho"'nuff daid. 'Lm-umph!" sezee, "look lak dat medincin ain "gree wid Mistah Wolf well $z$ he think fer. Dat $z$ a monstous big pill he try ter swoller. but w'en folks come "long yer an' fin' him dey kain't l, lame us. fer dey"ll see de "simmons stickin' ter his mouf, an' den dey"ll know he wuz dat big a fool he ain "kin tell de diff"n "twix" "simmons an' flint rocks. Le's we hurry up an git outen dis, but 'fo' I goes I gwine cut off his years an' tek 'em home ter my fambly, 'kase dey ain' gwine b'lieve hit lessen dey sees hit."

"De fambly wuz might'ly sot up orer de killin' er Mistah Wolf, an' Posum tuck ter wearin' de years stuck on orer his own. Dat huccome de wolfs ter 'spicion who kilt der daddy, an dar come mighty nigh bein" trouble.

"I done fergit ter tol" you dat Wolf had black years, an dat w' de Injun folks, urer sence 'Possum bin wearin dem borryed years, calls him 'de animuel wid de black years. My daddy he wuz a Injun. an he done tol' me de tale an' de name er de 'possum in ('hurrykee, but hit soun" so curisome I done fergit hit. An' now you-all hes' run 'long home. mos' time fer yo' dinner, an' lemme look atter de bugs on dish yer 'baccypatch er mine." 


\section{HOW IIR. TERRAPIN LOST HIS BEARD}

The "cook-house" stood at some little distance from the "big house," and every erening after supper it was full of light and noise and laughter. The light came from the fire on the huge hearth, above which hung the crane and the great iron pots which Eliza, the cook, declared were indispensable in the practice of her art. To be sure, there was a cook-store, but 'Liza was wedded to old ways and maintained there was nothing "store cooked" that could hope to rival the rich and nutty flavor of ash cake, or greens "b "iled slow an' long over de ha'th, wid a piece er bacon in de pot."

The noise and laughter came from a circle of dusky and admiring friends, for Aunt 'Liza was a great favorite with everybody on the plantation, and though hunchbacked and homely, had, nevertheless, had her pick, as she was fond of boasting, of the likeliest looking men on the place; and though she had been twice wedded and twice widowed, aspirants were not wanting for the position now racant for a third time. Indeed, not long before, a member of the family, on going to the cook-house to see why dinner was so late, had discorered one Sam, the burly young ox-cart driver, on his knees, pleading very earnestly with the elderly and humpbacked little cook, while dinner simmered on and on, unnoticed and 


\section{HOW IIR. TERRAPIN LOST HIS BEARD}

forgotten. Then remonstrated with she said that she was "bleeged ter have cotin' times ez well ez de res' er folks," and intimated that in affairs of the heart these things were apt to happen at any time or place, and that if a gentleman chose an inopportune moment " "twan ther fault," and no one could, with any show of reason, expect her not to pay attention to him. She ruled everybody, her white folks inchded, though just how she did it no one could say, unless she was one of those commanding spirits and born leaders who sometimes appear eren in the humblest walks of life. It is possible that her uncommonly strong will compelled the affections of her male admirers, but it is also possible that she condescended to flatter, and it is certain that she fed them well.

One night, between supper and bedtime, the children heard the sound of a banjo proceeding from the cookhouse. They had nerer rentured into Aunt 'Liza's domain before, but the plinketty-plunk of the banjo, the sound of patting and the thud of feet keeping time to the music drew them irresistibly. Annt Nancy was there, in the circle about the embers, as was also her old-time foe, Aunt 'Phrony, and the banjo was in the hands of Tim, a plow-boy, celebrated as being the best picker for miles around. Lastly, there were Aunt 'Liza and her latest conquest, Sam, whose hopes she could not have entirely quenched or he would not have beamed so complacently on the assembled company.

There was a hush as the three little heads appeared in the doorway. but the children begged them to go on, and so Tim picked away for dear life and Sam did a 


\section{AT THE BIG IIOLSE}

wonderful double-shufle with the pigeon-wing thrown in. Then 'T'im sang a plantation song about "Cindy Amn" that ran something like this:

I'se guine doun ter Richmond,

I'll tell you w'at hit's for:

I'se guine down ter Richmond

Fer ter try an' end dis war.

Refrain: An'-a you good-by, Cindy, Cindy,

Good-by, Cindy Ann;

An'-a you good-by, Cindy, Cindy, I'se guine ter Rappahan.

I oon ma'y a po' gal, I'll tell de reason w'y:

Her neck so long an' stinny

I'se'feard she nuier die.

Refrain.

I oon ma'y a rich gal,

I'll tell de reason $w^{\prime} y$ :

Belase she dip so much snuff

IIer mouf is nuerer dry.

Refrain.

I ru'rr ma'y a young gal,

A apple in her han',

Dan ter ma'y a widdy

Wid a house an' a lot er lan'.

Refrain 


\section{HOW MR. TERRAPIN LOST HIS BEARD}

At the reference to a "widdy" he winked at the others and looked significantly at Sam and Aunt "Liza. Then he declared it was the turn of the ladies to amuse the gentlemen. Aunt Nancy and Iunt 'Phrony cricd. "Hrsh! Go way man! W"at lien we-all do? Done got too ol fer foolishness: leave dat ter de gals:" But "Liza was not inclined to leave the entertainment of gentlemen to "gals." whom she declared to be. for the most part. "wuf"les trunnel-baid trash."

"Come. come. Sis" 'Phrony, an' you, too, Sis" Sancy." said she. "You knows dar ain' nu re pusson on de place kin beat you bofe in der marter ur tellin' tales. I ain' nuver have de knack mrof. but I knows a good tale wen I rears hit, an I bin gittin mysef fixed fer one urer sence rou comed in."

The children added their petitions, seconded by Tim and Sam. Aunt Sancy looked as if she were feeling around in the dusk of half-forgotten things for a dimly remembered story. perceiving which the nimbler-witted Aunt 'Phrony made haste to say that she believed she knew a story which might please the company if they were not too hard to suit. They politely protested that such was far from being the case. whereupon she began the story of how the Terrapin lost his beard.

"Lm-umph!". snorted Aunt Nancr, "who urer year tell ur a tarrpin wid a by"ud!."

"Look-a-yer, ooman," said "Phrony, "who tellin" dis, me er you?" Iou spose I'se talkin "bout de li’l ol nokyount tarrpins dey has dese days? Naw. suh! I'se tellin" "bout de ol' time Tarr"pin whar wu a gre chieft an' a big fighter, an "w'ensomurer tu'rr creeturs come roun' an' try ter pay him back, he jes' drord his haid in 


\section{AT TIIE BIG HOLSE}

his shell an' dar he wuz. Dish yer ain' no ol' nigger tale, neener, dish yer a Injun tale whar my daddy done tol' me w'en I wan't no bigger'n Mirs Janey. He say dat sidesen de by "ud, 'Tarr'pin had big wattles hangin' down beneaf his chin, jes' lak de tukkey-gobblers has dese days. Him an' Mistah Wi'yum Wil'-tukkey wuz mighty good fren's dem times, an' Tukkey he thought 'Tarr'pin wuz a monst'ous good-lookin' man. He useter mek gre't 'miration an' say, 'Mistah Tarry-long Tarr'pin, you sut'n' $y$ is a harnsum man. Dar ain' nu'rr creetur in dese parts got such a by'ud an' wattles ez w'at you is.'

"Den Tarr"pin hed stroke down de by"ud an' swell out de wattles an' say, 'Sho! sho! Mistah Tukkey, you done praise dese yer heap mo'n w'at dey is wuf,' but all de same he wuz might'ly please', for dar's nuttin' lak a li'l bit er flatt 'ry fer ilin' up de j’ints an' mekin' folks limbersome in der feelin's.

"Tukkey git ter thinkin' so much 'bout de by"ud an' de wattles dat seem ter him ez ef he kain't git long nohows lessen he have some fer hisse'f, "kase in dem days de gobblers ain' have none. He study an' he study, but he kain't see whar he kin git 'em, an' de mo' he study de mo' he hone atter 'em. Las' he git so sharp set atter 'em dat he ain' kyare how he git 'em, jes' so he git 'em, an' den he mek up his min' he gwine tek 'em 'way f'um Tarr'pin. So one day w'en he met up wid him in de road he stop him an' bob his haid an' mek his manners mighty p'litely, an' he say, sezee, 'Mawnin', Mistah Tarry-long, mawnin'. How you come on dis day? I ain' hatter ax you, dough, "kalse you done look so sprucy wid yo' by'ud all comb' out an' yo' wattles puff' up. I wish, suh, you 


\section{IIOW MIR. TERRAPIN LOST IISS BEARD}

lemme putt 'cm on fer a minnit, so'st I kin see ef I becomes 'em ez good ez w'at you does.'

"ol' man 'Tarr"pin mighty easy-goin' an' 'commodatin', so he say. 'W'y. sut'n'y, Mistah Tukkey, you kin tek 'em an' welcome fer a w'iles.' So 'Tukkey he putts 'em on an' mosers down ter de branch ter look at hisse'f in de water. 'Whoo-ee!' sezee ter hisse'f, 'ain' I de caution in dese yer fixin's! I'se saw'y fer de gals now, I sut'n'y is, 'kase w'at wid my shape an' dish yer by 'ud an' wattles, dar gwine be some sho'-'nuff heart-smashin' roun' dese diggin's, you vear me sesso!'

"Den he go struttin' back, shakin' de by'ud an' swellin' out de wattles an' jes' mo'n steppin' high an' prancin' w'ile he sing:

\section{'Cle'r outen de way fer ol' Dan Tucker, You'se too late ter git yo' supper.'}

"Den he say, sezee, 'Mistah Tarr'pin, please, suh, ter lemme keep dese rer? I blieve I becomes em mo“n w'at you does, 'kase my neck so long an' thin seem lak I needs 'em ter set hit off mon wat you does wid dat shawt li'l neck er yo'n whar you keeps tuck 'way in yo' shell half de time, anyways. Sidesen dat, you is sech a runt dat you g'long draggin' de by'ud on de groun', an' fus" news you know hits 'bleeged ter be wo' out. You bes" lemme have hit. 'kase I kin tek good kyare ur hit.'

"Den Tarr"pin say, sezee. 'I lak ter' 'commodate you, Mistah 'Tukkey, but I ain' see how I kin. I done got so use ter runnin' my fingers thu de by'ud an spittin' orer hit w'en I'se settin' roun' thinkin' er talkin' dat I dunno how I kin do widout hit, an' I kain't git long, no- 


\section{AT THE BIG HOCSE}

how, widout swellin' up de wattles wen I git tetched in my feelins. Sidesen dat, I kin tek kyare er de by ud, of I is a runt; I bin doin it a good w'ile, an' she ain "wo" out rit. So please, suh, ter han' me over my fixin's.'

“ 'Not wiles I got any wind lef' in me fer rumnin"; sez de Tukkey, sezee, an' wid dat he went a-scootin', ol' man 'Tarrpin atter him, hot-foot. Dey went scrabblin' up de mountains an' down de mountains, an 'twuz pull Dick, pull deril, fer a w'ile. Dey kain't neener one uv em climb up ve y fas, but w'en dey git ter de top, Tukley he fly down an' Tarr'pin he jes' natchully turn over an' roll down. But Tukkey git de start an' keep hit. W"en Tarrpin roll to de bottom ur a mountain den he 'd see Tukkey at de top er de nex' one. Dey kep' hit up dis-a-way 'cross fo' ridges, an' las' Tarr'pin he plumb wo' out an' he see he wan't gwine ketch up at dat rate, so he gin up fer dat das. Den he go an hunt up de cunjerers an' ax 'em fer ter he p him. He say, 'Y'all know dat by'ud an' wattles er mine? Well, I done loan em to Mistah Wiyum Nil'tukkey, 'kase he wuz my fren' an' he done ax me to. An' now he turn out ter be no-kyount trash, an'wat I gwine do? You bin knowin' $I$ is a slow man, an' if I kain't git some he p, I hatter say good-by by"ud an' wattles."

"What are 'cunjerers,' Aunt 'Phrony?" said Ned.

"Well now, honey," said she, "I dumno ez I kin jes' rightly tell you, but deys w'at de Injuns calls 'medincinmen,' an' dey doctors de sick folks an' he ps de hunters ter git game an' de gals ter git beaux, an' putts spells on folks an' mek 'em do jes' 'bout w'at dey want 'em to. An' so dese yer cunjerers dey goes off by derse'fs an' has a confab an' den dey come back an' tell Mistah .6 
Tarrpin dat dey reckon dey done fix Mistah Tukkey dis time.

" "W"at rou done wid him?" sezee.

"We ain' ketch 'im,' dey ses. 'we lef" dat fer you. dat ain" ow" bizness, but we done fix him up so"t you kin do de ketchin ' ro "se "f.'

"W" "Wat has you done to him, den?' sezee.

"Śon.' dey ses. 'we done putt a lot er lill bones in his laigs, an dat gwine slow him up might'ly, an' we 'pends on you ter do de res', 'kase we knows dat you is a grett chieft."

"Den Tarr"pin amble long ’bout his bizness an' neener stop ner res ontwel he met up wid Tukliey onct mo: He ax fer his byon an wattles ag"in, but Tukkey jes' turnt an' stept out f'um dat, Tarrpin atter him. But seem lak de cunjerers thought Mistah Tarr'pin wuz faster'n w'at he wuz, er dat Mistah 'Tukkey 'z slower'n w'at he wuz, kase Tarr'pin ain' nuver ketch up wid him vit, an' w'ats mo', de tarr'pins is still doin' widout by 'uds an' wattles an' de gobblers is still wearin' 'em an' swellin' roun' showin' off ter de gals, steppin' ez high ez ef dem li"l bones wat de cunjerers putt dar wan't still in der laigs. an' struttin' lak dey ruz sayin' ter ev'y pusson dey meets:

\section{'Cle'r outen de way fer ol' Dan Tucker, You'se too late ter git yo' supper."}




\section{HOW MR. TERRIPIX LOST HIS PLUMAGE AND WHISTLE}

"Well," said Janey, as Aunt 'Phrony finished telling of the loss of Mr. Terrapin's beard, "I saw a terrapin the other day, and it didn't look as though it ever had had a beard or wattles. I thought it was real ugly."

"Law, chil"," answered the story-teller. "you kain't tell wat one'r dese yer creeturs bin in de times pas' jes' by lookin' at 'em now. W'y, de day's bin w'en ol' man Tarr'pin wuz plumb harnsum. He done bin trick' out er mo'n jes' his by'nd an' wattles, I kin tell you."

"Oh, please do tell us!" cried Janey, and little Kit came and leaned on her knees and looked up into her face and echoed, "Es, please to tell us."

Thus besieged, Aunt 'Phrony consented to tell how the Terrapin lost his plumage and his whistle.

"I done tol" you." said she, "Tarr"pin wuz onct a harnsum man, an' dat de sho'-'nuff trufe, fer he had nice, sof' fedders all orer his body an' a fine, big, spreadin' tail, an' his eves wuz mighty bright an' his roice wuz de cle'res' whustle you urer yearn. He wuz a gre't man in dem days, I tell you dat, an' his house wuz chock full er all sorts er fine fixin's. He had sof' furs ter set on an' long strings er shells fer moner, an' clo'es all imbroider' wid dyed pokkypine quills, an' he 


\section{HOW MR. TERRAPIN LOST HIS PLCMLAGE}

had spears an' bows an' arrers an' deer-hawns, an' I dunno wat all sidesen dat.

"In dem days de Quail wuz a homely. no-kyount creetur. wid sca cely any fedders, an' a shawt, stumpy tail, an' no voice wuf speakin' ur. He wuz po', too, an' nob'dy tuck much notuss ur him, jes' call him 'dat ar ol' Bob White,' an' he go wamnerin' up an' down de kyountry all by his lonesome.

"One day he come "long pas" Mistah Tarr"pin's house, an' he peek in thu de do". he did, an' w'en he see all de fine doin"s. seem lak he kain't tek his eve "way f"um de crack. Den he seed 'Tarr"pin comin' down de road home, an' he 'low ter hisse'f, he did, dat dish yer de harnsumes" man w'at he urer seed, an' he be puffickly sassified ef he cu'd look jes' lak dat. He git mo' an' mo' enviable ur "im an' tuck ter hangin' 'roun' de naberhood, peekin' an' peerin' in at 'Tarr"pin w'enuver he git de chanct. Las' he say ter hissef dat he jes' natchully 'bleeged ter have dem fedders an' dat tail an' whustle, but he ain' knowin' jes' how ter git 'em, so he g'long off ter ax de he p ur a wise ol' Wolf whar live 'way, 'way up on de mountain an' whar wuz one'r dem cunjerers I done tol' you 'bout. Ez he went 'long he wuz fixin' up a tale ter tell Wolf, an' w'en he git ter de kyave whar de cunjerer live he knock an' Wolf 'spon', 'Come in!' in sech a deep, growly roice dat li'l Quail felt kind er seery, an he feel mo' skeery yit w'en he go hoppin' in an' see Wolf settin' dar wid bones strowed all roun' him, an' showin' dem long. white toofs er his er'y time he open his mouf. But he perch hissef up in front er Wolf, an' he say in a roice dat wuz right trim'ly, 'Howdy, Uncle Wolf, howdy! I done comed all de way 


\section{AT TIIE BIG IIOT'SE}

up yer ter ax yo he'p, 'liase I knows dar ain' nair' nuir man on dis mountain whar knows half ez much ez wat rou does. Please. suh, tell me w'at ter do.'

" 'Bob White, you is a li'l ol' fool,' sez Wolf, sezee, 'how kin I tell you w'at ter do w'en you ain' tol' me wat 'tis you wants?

"Den Quail he git li"l mo' pearter, an' he try ter mek Wolf feel please'. so he say, 'Laws-a-mussy! Tncle Wolf, I done fergit dat. but I reckon I do so kase you is dat smart I thought you mought know widont me tellin'.'

“'Drap dat foolishness' sez Wolf, sezee, 'an' lemme know wat you comed atter.' But all de same he wan't too smart ner too ol ter feel please wid de flatt'ry; show me de man whar is: lots ur em gits ketched by dat, nuttin' mo' ner less," and here Aunt 'Phrony cast a scornful glance at Xancy, who answered it by a toss of the head.

"Well, den," she resumed, "Quail start inter de meanness he bin hatchin' up, an' he say, sezee, 'Tncle Molf, deys a man down dar below whar gittin' ter be dangersome. He's rich an goodlookin', an a gre chieft an' a sho'-'nuff fighter, an' he kin do 'bout w'at he please wid tu'rr creeturs. A man lak dat boun' ter w'k mischief. Now, suh, ef you sesso, "pears ter me hit be mighty good notion ter tek 'way his good looks an' dat pleasan' roice whar he uses ter 'suade de people wid, an' gin 'em ter some er de quict an' peace 'ble folks whar ain' all de time stickin' derse'fs ter de front an' tryin' ter lead de people. Now yer I is, you bin knowin' me dis good w'ile. an' you knows my numbility an' submissity, an' ef you mek me de one ter do de deed an' den give me de fixin's fer my trouble, I gwine feel dat I kain't ve'y well refuge 


\section{HOIN MR. TERRAPIN LOST HIS PLTMAGE}

'em.' Right dar he putt his haid on one side an' look up at Wolf michty meek an imnercent.

"Wolf he sily he gwine think "bout hit. an' he tell Quail ter (rome back in seren days an git de arnser. So Quail he go hippitty-hoppin down de mountains, thinkin he bin mighty smart, an' wumnerin' of he kin stan' hit ter wait seven mo' days befo' he rob po' ol' Tarrpin.

" Nolf he went off higher yit, ter de top er de mountain fer ter ax de pinion er seren urr wolfs mo older an wiser dan w'at he wuz. Dey talked an dey sputed toge'r fer seren dars an nights. Den Wolf come back an Quail mate hase up ter see him ag̃ in. He say Quail mus go ter Tarrpin s house at midnight an do jes' lak he tell "im to. er hit be wuser fer him. stidder better. Quail lissen an' say he gwine do jes' lak he tell 'im, an' wid dat he g*long off. Jes' at de stroke er midnight, w'en de bat: wuz a-flyin' an de squinch-owls hootin' an' de jacky-my-lantums trabellin up an" down, he knock on Mistah Tarr'pin's do' an gin out dat he wuz a trabeller whar comed a fur ways an' wuz pow'ful tired an hongry.

"Tarrpin wuz a kin' man, so he vited him in an' gin him sump'n ter eat an drink an' made him set down on de sof" furs. "kase he folt saw y fer any pusson so po' an ugly ez wat Quail wuz. Den he say, "You mus be tired atter yo journerin, lemme rub you a w"iles. He rub de ugly, rough creetur fer so long time, an' den Quail sez, sezee, 'You sut'n'y is kin', but I ain wanter tire you out. I is resed now, so please, suh, ter lemme rub you a li"l.' He rub an he rub Tarrpin wid one han', an' all de time he wuz rubbin' hisse f 


\section{AT TIIE BIG HOUSE}

wid de urr. Dat-a-way he rub all de fedders offen Tarr'pin onter his own sef. Den he rub down Tarr'pin's tail 'twel 'twan't nuttin' but a li'l roun', sharp-p'inted stump, an' at de same time he wuz rubbin' his own tail wid tu 'rr han' an' puttin' Tarr'pin's fine, spreadin' tail onter his own li'l stump. Hit wuz plumb dark, so 't Mistah 'Tarr'pin ain' see w'at bin done. an' sidesen dat he wuz pow'ful sleepy f"um de rubbin'. Den Quail say he 'bleeged ter lay down 'kase he mus' git him a early start in de mawnin'.

"Befo' sun-up he wuz stirrin' an' he say he mus' be gittin' 'long. 'Tarr'pin go ter de do' wid him an' den Quail say, sezee, "Mistah Tarrpin, I year you has a monstous fine whustle, I lak mighty well ter year hit befo' I go.'

" 'W'y sut'n'y' sez de Tarrpin, sezee, an' wid dat he whustle long an' loud. Quail lissen at him wid all his rears, an' den he say: 'Nell, dog my cats, ef I ain' beat! Yo' voice is de prezack match er mine.'

"'You don' sesso! lemme year you whustle,' sez Tarr'pin, sezee.

"'Dat I will,' sez Quail, 'but lemme go off li'l ways an' show you how fer I kin mek myse'f yearn', sezee. He sesso 'kase he'z gittin' mighty 'feerd dat Tarr'pin gwine fin' out his fedders wuz gone. So he go 'way off inter de bushes an' whustle, an' sho' nuff, 'twuz jes' lak Mistah Tarr'pin's roice. Den Tarr'pin try ter whustle back, but lo, beholst you! his roice clean gone, nuttin' lef' but a li'l hiss, an' hit done stay dat-a-way clean ontrel dis day. 'Twuz gittin' daylight, an' he look down ur a suddint an' dar he wuz! wid nair' a smidgin' uv a fedder on his back. IIe feel so bad he go inter de 


\section{HOW IIR. TERRAPIN LOST HIS PLCMLAGE}

house an cre ontwel his eves wuz so raid dat dey stared dat-a-way urer sence.

"Ien Mis" Tarrpin she say, 'Is you a chieft, er is you a ol ooman? Whyn't you go atter dat man an' gin him a lambastin' an' git back wat b'long to you?' He feel kind er 'shame', so he pull hiseef toge'rr an' go out ter see w'at he kin do. 'Fo' long he fin' out dat de cunjerers bin at whi, so he know he gotter have he p, an' he go an' git all tu'rr tarr'pins ter he p him. Dey went ter de ol' wolfs, de cunjerers. an' dey ses: 'We is a slow people an you is a swif' people, but nemmine dat, we drar's you-all to a race, an' ef you-all wins, den you kin hill we-all; an ef we-all wins, den we gwine exescoot rou. An' ef you ain' dast ter tek up dis dyar', den er'ybdy gwine know you is cowerds.'

'Co'se de wolfs tucken de dyar' up, an' hit wuz 'greed de race wuz ter be orer seben mountain ridges, an' dat hit wuz ter be run 'twix' one wolf an' one tarr'pin, de res' ter look on.

"Ir'en de day come, ol' Tarr'pin he tuck an' fix up dis trick; he git six urr tarr'pins whar look jes' lak him, an' he hide one away in de bresh on top ur each er de six mountains, an' he hide hisse f away on top er de sebent'. Jes' befo' Wolf git ter de top er de fus' mountain. de tarrpin whar wuz hidin dar crawl outen de bresh an' git ter de top fus' an' gin a whoop. an' went over a li"l wars an' hid in de bresh ag"in. Wolf think dat mighty cur'ous, but he keep on, an' 'twruz jesso at er'y one, an' at de las' ridge co'se Tarr'pin jes" walk hissef outen de bresh an' gin a gre't whoop ter let er ybily know he done won de race.

"Den de tarrpins mek up der min's ter kill de wolfs 83 


\section{AT TIIE BIG HOT'SE}

by fire, so dey pen em all in a big kyare on de mountain an dey bring bresh an wood an pile in front ur hit, a pile mos" ez high ez de mountain, an den dey set fire to hit. an' de wolfs howl an de fire hit spit an' sputter an' hiss an' erack an' roar, an' all de creeturs on de mountain set up a big cry an' rum dis-a-way an' dat ter git outen de fire: dey wuz plumb stracted. an' hit soun' lak all de wil beas'es in creation wuz turnt aloose an' tryin' w'ich kin yell de loudes:. But de tarr"pins jes" drord inter der shells an" sot dar safe an soun", an" watched de fire burn an' de smoke an" de flame rollin' inter de kyave.

"De wolfs dey howled an" dey howled an" dey howled, an' de li'l ones dey cried an 'dey cried an' dey cried, an' las' de ol' ones felt so bad 'bout de chillen dat dey 'gun ter kill 'em off so's't dey ain' suffer no mo'. W'en de tarr'pins see dat, dey wuz saw'y an' dey mek up der min's ter let de res" off, so dey turnt 'em aloose f"um de kyare. But lots ur em had died in dar, an" dat huccome dar ain' so many wolfs now ez dey useter be. Some wuz nearer ter de fire dan tu'rrs an got swinged, an' some got smoked black, an' dat w'y ontwel dis day, some wolfs is black an some gray an some white, an' some has longer, bushier tails dan tu'rrs. Dey got so hoarse wid all dat cryin' dat der voices bin nuttin' but a howl urer sence.

"Quail he year wat gwine on, an' he tucken hisse'f outen dat kyountry fas" ez his laigs cu'd kyar' him, so Tarr'pin nuver got hack de fedders ner de whustle, an' ef you goes out inter de fiel' mos" any day you kin see Quail gwine roun' in de stolen fedders an year him whustle: 
HOW MR. TERRAPIN LOST HIS PLEMAGE

'Bob White, do right! do right! Do right!' do right, Bob White."

jes" ez sassy ez of he bin doin' right all his days, an' ez ef he bin raised wid dat roice stidder stealin' hit way f"um ol' man Tarrpin." 


\section{MIS' CAT AND MR. FRISKI MIOUSE}

"Gre't king!" said 'Liza when Aunt 'Phrony had finished the story of Mr. Terrapin's losses; "gre't king! 'Tarr'pin sho' had trials an' tribilashuns, same ez we-all po' simners is bawn ter go thu wid in dish yer vale er tears. Dat sut'n'y wuz a fine tale, Sis' 'Phrony, an' now we gwine lissen ter Sis' Nancy, 'kase long ez I bin knowin' her she alluz got sump'n wuf yearin'. Ain' dat de trufe now ?" appealing to the circle and meeting with polite confirmation from everybody except Aunt 'Phrony, for it may be accepted as an axiom that there is not room enough for two profesional story-tellers at the same fireside, especially if they chance to be ancient foes.

"'Scuse me please, Sis' 'Liza, I'se feelin' kind er tizzicky dis evenin'," said Nancy, a little offended at not having had a hearing before.

'Phrony sniffed contemptuously. "Umph! tizzicky!" said she in an andible aside, "dat a new name fer hit. Whyn't she say she gittin' ol' an' losin' her 'memb ance?"

Aunt Nancy snorted and tossed her head defiantly. "Ol"! who ol'? Well, ef dat don' beat bob-tail! W'en you-all knows dat ooman wuz a li'l ol' no-kyount piccaninny, runnin' roun' yer wid her hy'ar stickin' out in pigtails all over her haid, long befo' I wuz bawnded. Sho! I reckon I 'members mo' tales dan w'at she is 


\section{MIS' CAT AND MR. FRISKY MOTSE}

uver year de names ur, so many dat I dumno whar ter begin, but I blieve I gwine gin you one dat tell bout a fool ooman whar place her pennunce on de wid ur a man an' fin' husse'f lef' out in de col"," here she looked significantly at Aunt 'Phrony, “an' de name er de tale is 'Mis" Cat an' Mistah Frisky-mouse.'

"Onct dey wuz a li"l mouse bin livin' down a dark hole fer a right smart ur a spell, an gnorrin' an' gnorrin' ter git thu inter de room whar folks wuz livin: Las' he git clean thu an' he skip out middle wars de flo' an' darne an' eaper an' kyar on, an' set up on his ha niches an' ju'k his haid, fus' dis-a-way an' den dat-a-way, an' drap. down on all fo's an' run inter de cornders, an' peek an' peer er'ywhars wid dem li'l bright eyes er his'n. Dar want no pusson 't all in de room, 'ccusin' a big ol' cat whar wuz snoogin' an' snorin' by de fire, an' he step so light he wan't 'feard er wakin' her. 'Holy Moses!' sezee ter hisse 'f, 'dish yer my chanct, sho' ez I'm a livin' sinner! Look out, ol' lady, any ooman dat go ter sleep over her dinner kain th kyount on hit bein' dar w'en she wake up: naw. malam. dinners too sca'ce fer dat; dey airi layin' 'roun' loose er'y day in de week. I mus' let out dish yer belt er mine er I ain' gwine be able ter crowd hit all in.'

"Wid dat he tiptoe up ter de plate whar settin" by de fire an' nibble away fer dear life on Mis' Cat's dinner. Den he go darnsin' roun' some mo', an' he feel so gaily an' so sot up 'kase he done finish de job er gnorrin', dat he say he blicre he gwine on a frolic, an' he jes' natchully 'bleeged ter have some whisky. He go sniffin' an' snuffin' roun', an' las' he spy a jimmyjohn er whisky settin' up on a high shef. But, bless you, he wan't 


\section{AT TIIE BIG HOLSE}

gwine let dat himner him, fer I ses to you, gemmen an' ladiz, dat ef a man's sot on gittin a dram, he gwine climb a mountain er dig down ter de baid-rock but w'at he gwine git de wufless truck, an' nuver stop ter think 'bout hit bein' any trouble, neener.

"So he skint up ter de she'f an' clim' ter de mouf er de jug an' perched on de rim an' 'gun ter sip de whisky. He dip down an' git a moufful an' den th'ow back his haid an' smack his lips an' lick de draps offen his whiskers. 'Lan”! lan”!' sezee, 'dish yer de stuff I bin jes' natchully honin' fer dis long time. Seem lak hit go prezackly ter de right spot an' limber me up might "ly in my feelin's.' Den de dram 'gun ter mount up in his haid an' he done fergot whar he wuz an' 'menced ter sing a snatch f'um a ol'-time drammin' song, whar runs dis-a-way:

\section{'Mammy tuck a big dram, Daddy tuck a whopper, When hit come ter my turn I hatter lick de stopper.'}

"Jes' den his footses slip an' down he go, ker-chug! inter de whisky. He spit an' he sputter an' scrabble roun' tryin' ter git out, but 'twan't no use. Gentermans! dat 'uz one time w'en he git his fill er whisky. 'My lan'! sezee ter hissef, 'dish yer's jes' de way you fin' mos' things in dis life: heap mo' easy ter git in dan 'tis ter git out. 'Pears lak dese white folks got mighty' li'l sense, puttin' der drams inter ol', slickry-sided bottles. How kin dey 'spec' mouses ter git in an' out er sech contrapshuns, I lak ter know.' He see he wuz 88 


\section{MIS' CAT AND MRR. FRISKY MIOLSE}

fillin' up so fas' dat sump'n gotter be done, so he squeak out at de top ur his roice: 'Mris' Cat! Oh, Mis' Cat! Hi, Nis' Cat! Please, ma'am, ter he'p me outen dis whisky!"

"Cat she open her eyes an' blink a li'l, kind er slow an' sleepy, an' las' she say: 'Is my years done 'ceive me, er is I year a moure? Mebbe I done dream hit.'

"Naw'm, dat you didn"! sings out Mistah Friskymouse. 'Twuz a sho"'nuff mouse, an' I'se him, up yer in de whisky, an I wants you, please maam, ter climb up an' he"p ne out.'

“"Well, ur all de imp'ence!' sez Mis' Cat ter husse'f, sez she, 'an' dat's de same li'l rascal whar done runned 'way f'um me mo' times dan I kin kỵount. Him ter call on me fer he"p!' Den she say out loud: 'Mistah Friky-mouse, I sut'n'y id lak ter bleege you bout dis, but I gotter tell you, I think hit sarve you right ter drown in dat whisky, 'kase you wuz stealin' de white folkses dram w'en you drapt in. Yas, I think hit be 'bout right fer you ter be lef" ter die in de midse er yo" sin, ef I mought 'spress myee d dat-a-way.'

"Mis' Cat wag her haid an' look sollum; she done fergit how many times she bin roguin' it in de white folkses cream-jug. She wan't de one whar git ketched dis time, so she ac' mighty pious orer Mistah House's thievin'.

"He baig an' he baig an' he baig, an' las' she say, 'Well, I he'p you out dis time, ef you promuss me you set by de fire an 'dry yo 'se'f good, befo' you go.' Right dar she wunk one eye at de fire an' smack' her lips an' sez she ter husse'f, sez she, 'I gwine git me a mouse dis trip, sho' ez shootin'? 


\section{AT TIIE BIG HOT'SE}

"In co'se Monse he gin de promuse, "kase 'twuz mighty nigh time"s-up wid him, yit he wan't so fur gone but w'at he wunk de one eye whar wuz still 'bore liquor an' ses' ter hisse'f, 'Tas'm, yas, ma'am, jesso, ma'am, but I have you fer ter know dat I wan't bawnded yistiddy ner de day befo', ner de day befo' dat, an' I ain' 'spec' ter die ter-day ner to-morrer ner de day atter.'

"Soon 'z he gin de promuss, Mis' Cat she lipt up on de she'f an' retched a paw down in de jimmyjohn an' fished 'im out, an' suz! he sut'n'y wuz a mis'able lookin' creetur, wid ery hyar on him plastered down close ter his li'l ol' lean body, an' de whisky drappin' f'um nose an' eyes an' mouf. She cu'd 'a et him den an' dar, but she wanter hare a li'l fun wid him fus', same ez cats alluz does wid de mouses befo' dey eats "em. Seem lak dey inj'rs 'em heap mo' ef dey lin skeer 'em good fus', an' den let 'em git mos' away, an' den kotch 'em ag'in, an' maul an' pull an' wool 'em roun' 'twel de mouses is glad ter be et up an' putt outen der mis'ry. So she tucken him an' putt him down by de fire, an' den she turn her back an' walk over ter tu'rr side whar she kin set an' watch him ter see dat he ain' git 'way. Suz! her back no sooner turnt dan Mistah Frisky-mouse jes' tucken his foot in his han' an' went clippin' orer ter de hole quicker 'n a streak er greast lightnin', an' Mis' Cat she look roun' jes' in time ter see de een' ur his tail gwine down de hole.

"'Hi, dar!' sez she, 'ain' you gin me yo" wu'd ter set by de fire ontwel you dry de whisky offen yo' hide? Is dis de way you keeps yo' wu'd? Does you call dat hon'able, atter I done he'p you out?'

"Mouse he whu'l 'roun' in de hole jes' so he kin poke 


\section{MIS" CAT AND MIR. FRISKY MOLSE}

his nose an eves out a li"l wars an' see her settin' dar wirl one han' lif" up. shakin' hit at him an' lookin' lak she plumb scannelize. He say. he do, 'Keep my wuid: Who? Me: Huh-uh, Mis Cat, heap ru rre lose my wid dan ter lose my life. sidesen dat, you knowed I bin drinkin'; deed rou done foun' me clean unner de infloons er whisky, an' you done yearn fo' now, 'kase you is no kitten, dat whisky meks mens mighty onre-

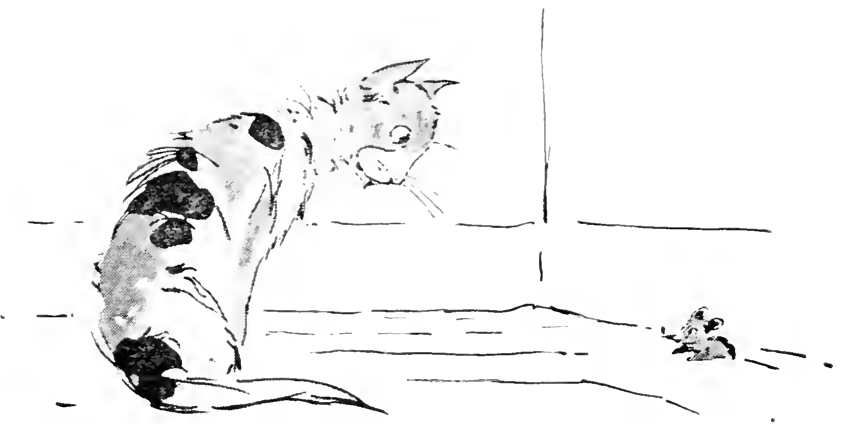

liable, dat hit do.' He git ter snickerin' an laughin' so't he kain't say nuttin' mo', so he go scootin' down de hole an' leave Mis' cat settin' dar wid one paw in de air an' her jaw drapt wide open.

“'De mis"able, ongrateful, owdacious li'l sinner' sez she. Jes' den Mistal Frisky-moune he scoot back fer a minnit an' see her settin' dar lookin' foolish, an' he laugh an' he rell an' he holler, an' jes' w'en she mek a dab at "im wid 'or paw, he turnt tail an'went creerin' down de hole, singin' back at de top er his voice: 


\section{AT THE BIG HOLSE}

\section{'Ho, ho! Mis' Cat, I thought you'z sticker' \\ Dan ter 'pend on de u'u'd ur a man in liquor." "}

"Dear me." said Janey, "what a naughty little mouse. Was he really, really tipsy ?"

"Sho!" said Aunt Nancr, "he wan't so tipsy but w'at he knowed dat Mis" Cat wuz atter him an' sot on ketchin' 'im, an' he knowed 'nuff ter git 'way f'um her, so I reckon he wan't so tipsy atter all. Dat's de way wid some wimmins." and here she rolled her eyes meaningly at Aunt "Phrony, "dey ain" got de sense ter hide hit w'en dey's tryin' ter ketch a man, an" de man he gits skeered an' quits, an' den de ooman goes an' putts de blame on some urr gal. I tell you, honeys, you kin run atter a man all you please, but you mus'n' let him 'spishun hit er de game's up.

"But I kain't stay yer runnin' on all night, an' dat sut'n'y is yo' maw I year callin'. Ef y'all don' g'long back ter de house, I boun' you she gin you a dose er w'at Paddy gin de drum; y'all know w'at dat wuz." 


\section{IIR. IIARE TRIES TO GET A WIFE}

One day the dhildren's mother told them that she was going to spend a few dars at a plantation some miles awar, taking with her Aunt Sancr, who was anxious to pay a little visit to a daughter living in that neighloorhood. Aunt 'Phrony, she told them, had promised to come and look after them during her absence.

"Oh please. mamma," they begged, "let Aunt 'Phrony take us nutting? She told us one day that she knew where there were just lots and lots of walnuts." So it was arranged that they should take a luncheon with them and make a day of it. Aunt 'Phrony being perfectly willing, for her Indian blood showed itself not only in her appearance, lut in her love for a free out-of-door life, and her fondnes for tramping. She would readily give up a day's work at any time to discharge some wholly insignificant errand which involved a walk of many miles.

The day was a bright and beautiful one in October, warm, yet with a faint nip of last night's frost lingering in the air. They made a fine little procession through the woods, Aunt 'Phrony leading, followed by children, a darky with baskets, her grandson "Wri'yum," and lastly the dogs, frisking and frolicking and darting away every now and then in pursuit of small game. A very weary and hungry little party gathered about the baskets at 


\section{AT THE BIG IIOT'SE}

onc o'clock, and three little pairs of white hands were stained almost as brown as those of Aunt Phrony and William. But everybody was happy. and there was a nice pile of walnuts to go back in the large bag which William had brought for the purpose. The dogs sat around and looked longingly on. a squirrel frisked hastily across a log near by, the birds chattered in the trees high above and looked curiously down on the intruders, and presently a foolish hare went scurrying across the path, so near the dogs that they sat still, amazed at his presumption, and forbore to chase him.

"Hi! there goes 'ol' Hyar" "." shouted Ned; "I'm going to see if I can't catch him." But he soon gave up the hopeless chase.

"Was that your 'ol' Hyar", Aunt 'Phrony; your ol' Hyar' you tell us all about?" asked little Kit.

"Bless de chil":" said she. "Naw, 'twuz de ol', ol' Hyar' I done tol' you 'bout, de gre 't-gre't-gre't-sump'nru'rr grandaddy er dis one. I reckon."

"Aunt 'Phrony," said Janey, "couldn't you tell us some more about the old hare while we sit here and get rested :"

"Now de laws-a-mussy," said 'Phrony, "ef we gwine 'mence on de ol' tales I reckon I mought ez well mek up my min' ter spen' de res' er de day right rer on dis spot," and she leaned back against a pine tree and closed her eyes resignedly. Presently she opened them to ask. "Is I urer tol' you 'bout de time Mistah Hyar" try ter git him a wife? I isn'? Well, den, dat de one I gwine gin you dis trip. Hit happen dis-a-way: Hyar' he bin flyin' all 'rom' de kyountry fer right long time, frolickin' an' cuttin' up, jes' a no-kyount bachelder, 


\section{MR. HARE TRIES TO GET A WIFE}

an' las' he git kind er tired ur hit, an' he see all tu'rr creeturs gittin' ma ied an' he tucken hit inter his haid dat 'twuz time he sottle down an' git him a wife; so he primp hissef up an' slick his hyar dom wid bargrease an' stick a raid hank'cher in his res'-pockit an' pick him a button-hole f"um a lady's grarden. an' den he go co tin' dis gal an' dat gal an' tu'r gal. He 'mence wid de good-lookin" ones an" wind up wid de ugly ones, but "twant nair" one dat "ur lisen to "im. "kase he done done so many mean tricks an" wuz sech a hyarumskyarum dat dey wuz all 'feard ter tek up wid “im, an’ so dey shet de do' in his face w'en he git ter talkin' sparky. dough dar want no pusion cuid do dat sort er talkin' mo' slicker 'n w'at he cu'd. But he done gin de creeturs jes' lill too much 'harishness, so 'twan't no use.

"He think de marter all orer an" he say ter hisse"f: 'Tem fool gals dunno wat dey misin', but ef dey spose I gwine gin up an stay single, dey done fool derse'fs dis time. I ain grine squatulate wid em ner argyfy ner beg no mo', but I gwine whu'l right in an' do sumpin.'

"Atter he study a w'ile he slap one han' on his knee, an he low, he do: 'Dat's de ticket! dat's de ticket! I reckon dey'll fin' ol man Hyar' ain' sech a fool ez he looks ter be, atter all.'

"He go lopin' all roun', learin' wrd at er'y house in de kyountry dat a big meetin' bin hilt an' a law passer dat ev'yb'dy gotter git ma“ied. roung an' ol', rich an' po', high an' low. He say ter hisse'f. 'Ev'yb'dy, dat mean me, too, so dish yer whar I boun' ter git me a wife.' 


\section{AT THE BIG HOLSE}

"De ereeturs place der pennance on him, dough he done tucken 'em in so often, an' on de 'pinted day dey met toge'rr; de gals all dress' up in der Sunday clo'es an' de mens fixed up mighty sprucy, an' sech a pickin' an ' choosin' you nuver see in all yo' bawn days. De gals dey all stan "up in line an' de men go struttin' mighty biggitty up an" down befo 'em, showin' off an' makin' mamners an' sayin', 'Howdy, ladiz, howdy, howdy!' An' de gals dey'd giggle an' twis' an putt a finger in de cornders er der moufs, an' w'en a man step up ter one ur 'em ter choose her out, she 'd fetch "im a li'l tap an' say, 'Hysh! g'way f'um yer, man! better lemme 'lone!' an' den she'd giggle an' snicker some mo', but I let you know she wuz sho" ter go wid him in de een".

"All dis time Hyar" wuz gwine up an' down de line, bowin' an' serapin' an' tryin' ter mek hisse'f 'greeable ter ev'rb'dy, even de daddies an' de mammies er de gals, whar wuz lookin" on f"um tu'rr side. Dar wuz whar he miss hit, 'kase w"ile he wuz talkin' ter de mammy ur a mighty likely li'l gal whar he think 'bout choosin', lo an beholst, de choosin' wuz all over, an' w'en Mistah Hyar' turnt roun' dar want nair' a gal lef', an' ev'y man have a wife asseptin' him.

“Den dey hilt a big darnsin' an' feastin', an' ev'yb'dy wuz happy an' in a monst'ous good humor, de gals 'kase dey done got ma "ied, an' de paws an' de maws 'kase dey done got redd er de gals, - er 'yb'dy "scusin' Hyar". Dey mek lots er game ur "im, an" w'en dey darnse pas', dey sings out: 'Heyo! Mistah Hyar', huceome you ain' darnse?' 'Bring yo' wife. ol' man, an' jine in de fun!' 'Hi! yi ! Mistah Hyar', you done ma'y off er'yb'dy else an' stay single yo 'se'f? Well, dat de meanes' trick you 


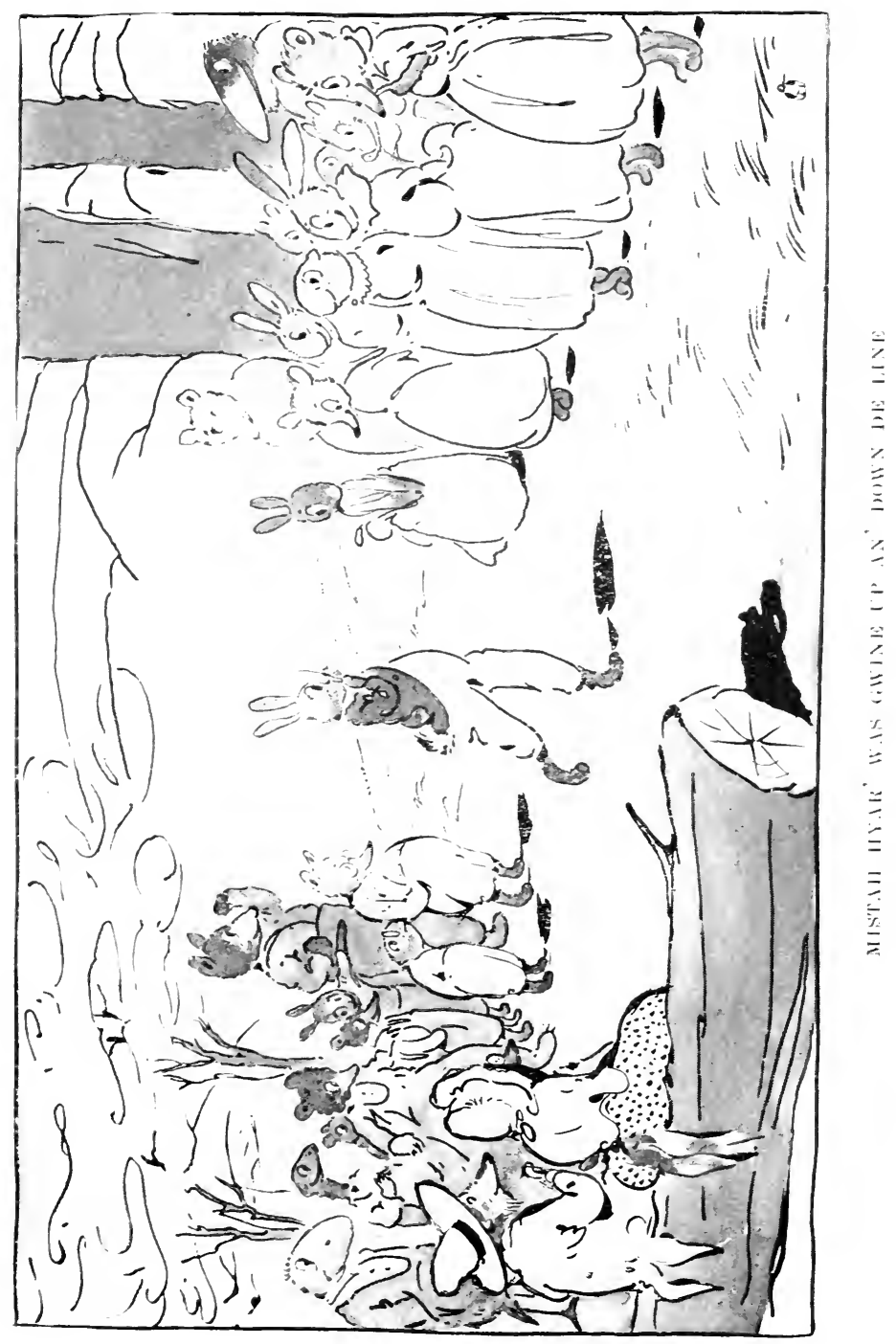





\section{MIR. HARE TRIES TO GET A WIFE}

done played us yit! 'tain' fair!' An' ley snicker an' run on 'twel Hyar' wish he ain' nuver year de wh'd ma'y.

"Atter w'ile dey got tired er darnsin" an" tucken der new wifes an' went off home leavin' Hyar' all by hisse'f, an' I tell you he feel right lonesome. IIe git a bad spell er de low-downs an' go squanderin' roun' thu de woods wid his years drapt an' his paws hangin' limp, studyin" how he kin git revengemint. Las" he pull hisse'f toge'rr an' he say: 'Come, Hyar', dis ain' gwine do. Is you done fool er'yb'dy all dese ears an" den let yo'se"f git fooled by a passel er gals? Naw, suh! I knows wat I gwine do dis rey minnit. Ef I kain't git me a gal. I kin git me a widdy, an' some folks laks dem de bes, anyhows. Ef you ma'y a widdy, she got some er de foolishness knock' outen her befo' you hatter tek her in han'?

"Wid dat he step out ez gaily ez you please. He go an' knock at de do" ur er' $y$ house. an "w'en de folks come ter de do' dey say. "W'Y. howdy, Mistah Hyar', whar you bin keepin yo"se'f all dis time?" He say, he do: 'Oh, I bin tendin' ter de 'fairs er de kyountry, an' I is sont unter you ez a messenger. I is saw y ter tell you dey done hilt nu'rr big meetin' an mek up der min's de worl' gittin' too many creeturs in hit, so dey pass de law dat dar mus" be a big battle, an "you is all ter meet toge "rr at de "pinted time, an" each man mus" fall 'pun de man nex' him an' try fer ter kill 'im.'

"De creeturs assept dis wid submissitty, dey ain" 'spicion Hyar' 't all. On de 'pinted day dey met toge'rr, an' each wuz raidy ter defen' hiseef. Hyar' wuz dar lak all de res'. an' ef you'd 'a seed all de spears an' bows an' arrers he kyarry, an' all de knifes stickin' in his 


\section{AT THE BIG IIOLSE}

belt, you'd 'a thought he wuz de bigges" fighter dar. But sho! W"en de fightin begin, hit wu far'-you-well, gentermans! "Twan't no Hyar" dar: he jes" putt out tight 'z he kin go. I'"en dey see him goin' dey sing out: 'Hi, dar! Jistah IJyar" Whar rou gwine? Whyn't you stay wid we-all an' fight dis out?'

"Hyar" ain" stop ter talk, he jes' look roun' over his shoulder w'iles he 'z runnin' an' he say, sezee: 'De man I wanster kill, he done rumed "way an" I'se atter him, hot-foot, de mis'able cowerd. Kain't stop ter talk; git outen my way, er'yb'dy,

'Cle'r de track, fer yer me comin', I'se ol' Buster whar heep things hummin'.'

"W'en de battle wuz orer, de creeturs miss Hyar', an" dey say he mus' be 'mongs' de kilt. so dey go roun' lookin' at de daid. but 'twan't no Hyar' dar. Dey hunt ev'ywhar fer him an' las' dey foun' him squattin' in de bresh. tremlin' ez ef he have de ager an' nigh mos' skeert ter def:. Dey drug him outen dat an' dey ses: 'So dish yer's Buster whar keep things hummin'! Well, we gwine mek you hum dis time, sho' 'nuff. You putts we-all ter fightin' an' gits heap er good men kilt off, an' yer you settin' tuck 'way safe in de bresh. We gwine hole you 'sponsible fer dis, an' we gotter know de wharfo's, too.'

"Den ol' Hyar' he up an' 'fess he done de hull bizness so's't de kyountry mought be full er widdies an' he git him his pick fer a wife, fer he "lowed widdies wan't gwine be so p'tickler ez de gals. De creeturs jes' natchully hilt up der han's at him, dey wuz plumb outdone. 


\section{IRR. HARE TRIES TO GET A WIFE}

'De owdacious vilyun!" dey ses. "Te boun' ter exescont him on de spot an" git shed ur "im onct fer all." But he baig mighty hard an' some ur 'em think he be wuss punish ef dey jes' gins 'im a good hidin' an' lets 'im live on alone, a miscible ol bachelder, widout no pusson ter tek notus ur "im, kase in co"se none er de widdies wuz gwine ma'y a cowerd."

"Why, dunt "Phrony," said Ted, "he must have found a wife at last, for how about Mis' Molly Hyar" ?"

"Shucks!" said she, "is I urer tol' you 'bout Mis' Molly Hyar"? Naw. suh. she b'longs in dem ol' nigger tales whar Nancy tells you. De Injun tales ain' say nuttin' 'bout no wife er his'n. He wuz too gre t a fighter an' too full er 'havishness urer ter sottle down wid a wife; an' now lemme finish de tale.

"Dey gin him a turr"ble trouncin' an' den turnt him aloose, an' stidder gittin him a wife he got him a hide dat smart f"um haid ter heels: but w'en my daddy tell dat tale he useter een' her up dis-a-way, 'An' mebbe Hyar' git de bes' ur 'em, atter all. 'kase w'en rou git a hidin', de smart s soon orer, but w'en you git a wife. de mis'ry done come ter stay.'", 


\section{HOW IRR. HARE LOST HIS HORNS}

"Well, I say!" said Ned, when Aunt 'Phrony had finished telling of $\mathrm{Mr}$. Hare"s failure to get a wife, "who"d think such a little old thing as a hare could make so much mischief :"

"Li"l ol' thing! him? not in dem days, chil'," said the old woman. "Ain' I tol' you, tu'rr' night, dat de creeturs wan't lak dey is now? W'y, Hyar' he wuz a big man, I tell you, an' he had hawns: yas hauns, sho''nuff' hawns, jes' lak de deers has. 'Deed, de deer-hawns useter b'long on his haid, 'twel tu'rr creeturs dore tucken 'em 'way f'um 'im. I gwine tell you bout dat ef you promuss not ter pester me 'bout no mo' tales fer one w'ile."

Of course the children promised, and the old woman, with head thrown back against the tree and a dreamy look in her eves, as if she were contemplating those far-off days when the hare was the great hero of many a tale told by the camp-fires of her father's people, began the story of the lost horns.

"I done tol' you." she commenced, "dat Hyar' useter wear hawns, an' dis huccome he ter lose 'cm. At fus' he ain' have none, but he git ter be sech a big man an' sech a smart man dat de creeturs look up ter him might'ly, an' dey wanter do sump'n ter show der regyards uv 'im, 'kase äish yer wuz befo' he git so trickish; 


\section{HOW MRR. HARE LOST HIS HORAS}

so der have wat dey call a kyouncil an' talk hit all over an agree ter gin him a harnsum pair er hawns, thinkin dat sech a smart creetur boun' ter mok good use uv 'em. So der call him up an' dey ses, "Mistah Hyar', we done 'cide ter putt dese hawns on you, an' we wants you ter 'member dat we nurer putts 'em on any but tiptop men an' natchel-bawn rumners, an' we 'spees you ter keep up ro" good name in dis marter. Now lessee w'at you kin do wid 'em.'

"Dey gin him de wu'd an" way he went, but mebbe de hawns mok 'im feel sort er onbalance in de haid er somehows outer kelter, 'kase stidder rumnin' lak he useter he went wid a sort er hop, skip an' jump, an' in de place er gwine up an' down de medders whar him an' Mistah Dapple-deer useter kite roun' toge'rr, mighty good fren`s, he jes' tucken ter de bresh an' dey ain' see him no mo' fer so long time.

"De creeturs say ter one nu'rr. "Tr'at in de name er goodness done comed orer Mistah Hrar"? He useter live out in de open 'long wid Mistah Deer, 'havin' hisse'f lak a 'sponsible pusson, an' now he done quit we-all, an' lawd on'y knows whar he is at. Look-a-yer, Mistah Dapple-deer, we gwine 'p'int you ter hunt up yo' fren' an' bring 'im back ter dis krouncil. Kin we 'pend on you fer dis, suh?'

"Deer he say: 'Well, I ain' mek no promuss; dat ain' my way. Makin' an’ breakin' is bofe pow'ful easy; but ef I sesso, den I gwine do so, an' I say dis much: I gwine do w'at I kin, but mebbe you has notuss dat follis is 'bout de mos' onsu 't'n truck w'at der is: I mought arnser fer Mistah Iyyar' yist'd'y, but I kain't tell w'at he gwine do to-morrer.' 
"Well, suh, he go an" he hunt an' he hunt an' he hunt. Fus" he look in de medders, no Mistah Hyar"; den he look in de woods, no Mistah Hyar"; den he tuck ter lookin' in de bresh, an dar lie wuz, settin' up on his 'hime laigs an' gnorrin' a locus' twig, jes' ez onconsarn' ez ef he bin livin' dar all his life."

Ted interrupted to say, "What in the world did he want to gnaw a locust twig for?"

"Oh, dat wuz fer his front toofs. He teck a twig er de black locus', 'kase dat good an' hard, an' he gnor an' he gnor, ter keep his front toofs groun' down; fer ef he ain' do dat dey'd grow so long he cu'dden use 'em, 'deed dey would. Well, he wuz grindin' way on de twig an' he year Deer comin' an' he say, 'Howdy, Mistah Dapple-deer, howdy', widout eren so much ez turnin' his haid.

" "Well, ur all de news!" sez Deer, sezee, 'w'at you doin' yer so fur f'um de place whar you b'longs ter?'

"'Jes' gittin' my toofs in good fix,' sezee, gnorrin' on ez ef he nurer 'spec' ter git nu'rr locus' twig.

"'Come 'long outen yer,' sez Deer, 'dis no place fer a man er yo' breens an "unnerstannin'; come out yer in de open an" show yo'se'f. W'at mek you wanter skulk an" hide yo"se'f in de bresh?"

"'Tired er de open,' sez Hyar", sezee, 'tired er rumnin' races; tired er gwine all 'roun' creashun ter fin' vittles. Gwine stay right in yer whar I kin fin' 'em handy; gwine teck things easy f ${ }^{\prime \prime m}$ dis on. Ef dese yer breens er mine whar you talk "bout is good fer anything. dey tell me dat w'uk don' 'gree wid me, nohow.'

"Deer he 'suade an' he 'suade, but 'twan't no use, an' las' Hyar' git kind er riley an' he say, he do: 'Don' you 


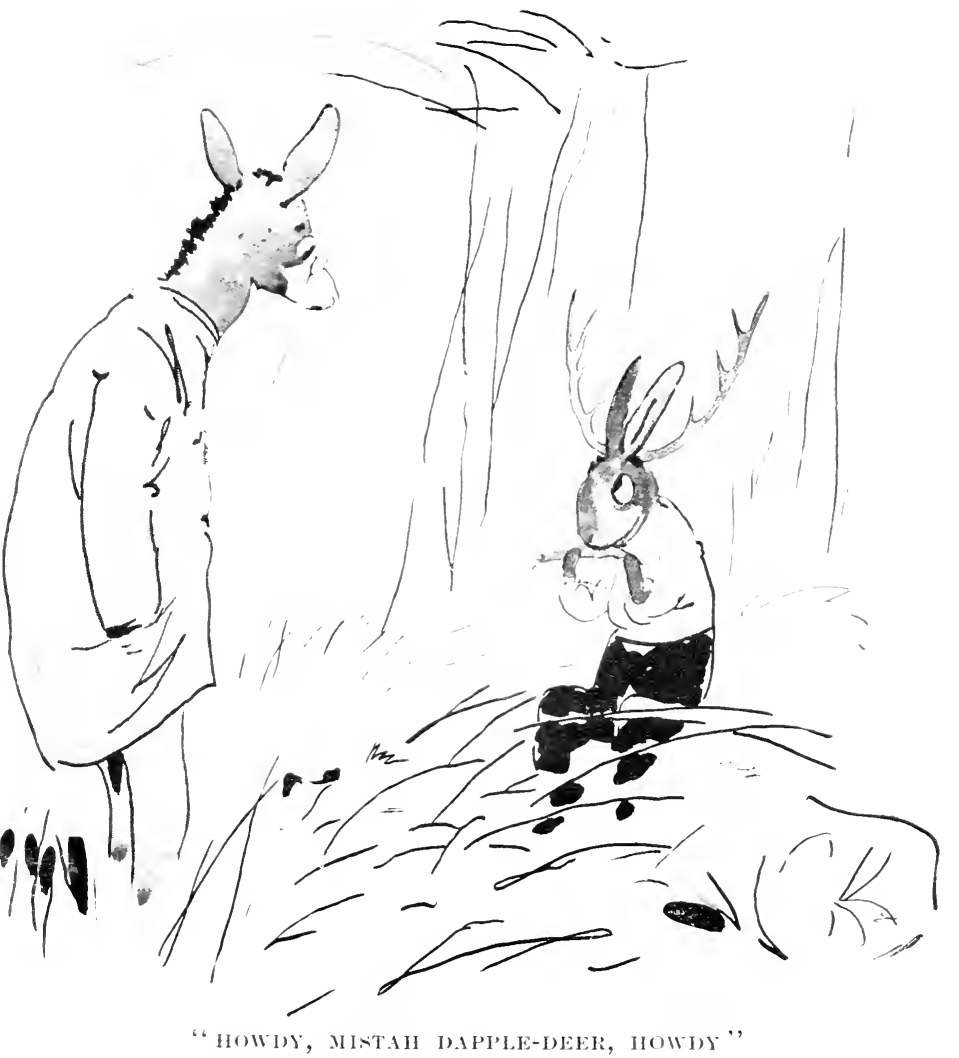





\section{HOW MR. HARE LOST HIS HORAS}

'suade me no mo'. My min' done mek up. You better lemme "lone. "kase now I got me dese hawns I is lind er dangersome, an' you bes' not git in a fist-an'-ckull fight wid me, "kase ders no tellin' whar you mought bring up. on de p"int er dese hawns. I dunno: I ain "sponsible fer 'em,' an' wid dat he shuk de hawns serrigrous at po' ol' Deer.

" "Well. ef dat ain' de gre'tes' howdy-do!' sez Deer, sezee. 'An' dish yer wuz de man I done place my 'pennance on an think wuz my fren'! Temmine, g'long an' do de way you wanster; I done wath my han's er de bizness. De fool-killer better come dis "way, dough, right quick, 'kase dars a good size job waitin' fer him right close ter dese diggin's, an' I jes' 'bout b'lieve I gwine leare wu'd fer him whar ter call, ez I g"long back.'

"Wid dat he sainter off a li"l wars, thinkin" mebbe Hyar' gwine foller. Yaw suh! he jes" sot dar an" went on gnorrin'. W'en Deer see dat, he succle 'roun' thu de woods a piece an' come up behime ol' Hyar' an' grab 'im back er de hawns an' go lopin' off wid 'im ter de kyouncil.

"'Set down de pris'ner,' dey ses ter Mistah Deer. Deer putt him down an' he sot dar jes' ez ca'm ez you please, still gnorrin' on a li'l locus' twig he bringed wid 'im. 'Mawnin', sezee. 'gemmen, mawnin'. Hope I sees y'all right well. Y'all lookin' tol'ble, hope you feclin" dat-a-way, dough "look" an" "feel" spute wid one nu'rr mighty often in dis worl'. Yer me, all store up wid de rheumatiz, dough I 'spec' you oon b'lieve hit jes" ter look at me.'

"De kyouncil ain' pay no 'tention ter his gwines-on. 


\section{A'T TIIE BIG IIOTSE}

'Pris'ner, has you anything ter say fer yo'se'f?' dey ax "im.

" "Plenty, sezee, cool ez a cowcumber, 'lid any pusson nver see de time w'en I kain't wag my tongue wid de bes'? But ef you mean 'bout dem hawns, I ain' got nair' wu'd ter say, 'ceptin' dat you done putt 'em on me too suddintly. Seem like dey kind er goes ter my haid an' mek me wanter do diff'nt f'um w'at I uver has befo'. I'all 'd orter let dem hawns grow on me, li'l at a time, so 's't I monghter got use' to 'em.' Den he set back on his ha'nches an' look lak 'twan't none er his bizness an' he wan't kyarin' w'at dey done wid 'im.

"De kyouncil lean der haids on der han's an" study 'bout de marter, an' las' dey ses, 'Mebbe we done mek too much has'e in dis bizness, so we ain' gwine be ton hard on you, but we p'intedly ses ter you dat you kain't keep dem hawns; we 'bleeged ter teck 'em way f'um you an' gin 'em ter some pusson dat gwine mek good use uv' 'em.'

"Ol' Hyar' he say, sezee: 'Gemmen, dat suit me to a hya'r, "kase dey is too big fer dis chil" an' mek him feel kind er top-hairy.'

"So dey ondone 'em offen Hyar', an' den dey call up Deer an' ses, 'Mistah Dapple-deer, you done stuck by de kyouncil an' you done done yo' juty in the place whar we putt you an' you has foun 'Mistah Hyar' an' bringed him back ter we-all. an so now we gwine gin you dese hawns fer de reward er yo' sarvices.'

"So Deer he go tippin' up an' kneel down an' dey fasten de hawns on 'im. I'm-umph! No sooner done dan he scrabble ter his footses an' gin a boun' an' went jumpin' off inter de bresh. an' dar whar he bin livin' 


\section{HOW MR. HARE LOST HIS HORNS}

urer sence. Onct in a wiles you hin see him cross de open, but he do hit in a mighty big hurry, I tell you, jes' two er thee monst ous jumps an' he git clean 'cross a big fiel', an' even den he sca'cely urer cross lessen de dogs is atter 'im, an' he ain' see no urr way ter git outen dey road.

'Hyar' he squat dar an' look atter Mistah Deer, an' he laugh an' he laugh, an' he laugh 'twel he fall over an' roll on de groun'. Den de krouncil dey ses. 'Mistah IIyar', we wants you ter set up, suh, an' quit dis "havishness; dar ain' muttin' ter laugh 'bout in dis bizness, 'kase de kvouncil done los' nu'rr goor member.' ol' Hyar' he set up an he bow an' he scrape an he say, he do, "Scuse me, gemmen, but I hatter laugh w'en I think 'bout de effec' er dem hawns on sech a well behave' pusson ez Dapple-deer. Dish yer gwine l'arn y'all, I reckon, dat 'tain' safe ter pile up de honors on a man's haid too suddintly. dey kain't stan' hit. Y'all please 'scuse me ef I run 'long atter Dapple-deer, "kase I done had a tas'e er livin' in de bresh an' you mus'n' spec ter see me often out yer in de open. So long, gemmen. so long!' He run off inter de bresh an' stay dar, befo' dey kin say Jack Robumsum, an' dat w'at all de hyar's bin doin' urer sence." 


\section{WIY THE TITIOOSE HAS A BLUNT TONGUE}

The children sat perfectly quiet after Aunt 'Phrony had finished telling them about how Mr. Hare lost his horns. for they remembered that they had promised not to "pester" for more. But the story-teller was in an amiable mood, and, without solicitation, went on to relate another tale, in which MIr. Hare figured again.

"Onct," she said. "ol' Hyar' wuz livin" 'way off in de mountains, so high up dat he cu'd look out over de kyountry an' see er'ything an' know jes' 'zackly w'en anything wuz gwine on. Ery time he seed a big smoke he knowed a fire bin built an' dat sump'n er nu'rr wuz in de wind, so he 'ud jes' light out fer de place, an' w'en he git dar he alluz start up some trouble er ru'rr.

"One time dar wuz a heap er sickness 'mongs" de people, an' so dey went ter wu'k an' had a medincin'darnse."

The little folks wanted to know what a medicine dance might be.

"Oh, honeys!" Aunt 'Phrony exclaimed, "I tell you a medincin'-darnse wuz one big time. dat hit wuz! My daddy done tol' me all 'bout hit. Dey mos' in gin'ly hilt de darnse in de fall, dem Injun folks, but sometimes 'twuz in de spring, an' ef dar wuz any dese yer ketchin' diseases 'roun', dey hilt hit mos' any time ter keep off de sickness. My daddy's people b'lieve ev'ything dat 106 


\section{WHY THE TITIOUSE HAS A BLINT TONGLE}

grows got some use in sickness. Dey ses dat long, long time ago de anermuels got toge'rr an' made up der min's dat dar wuz too many mens in de worl', so dey putt der haids toge'rr and got up a pow'ful lot er discases fer ter kill off de people. Den all de growin' things, w'en dey got de news er dis, dey ses, "We-all is de fren's er men, we gwine gin 'em w'at he'p we kin in dis marter.' so each er de trees an de bushes an' de yarbs an' de grasses an" de mosses dey 'greed ter gin a kyore fer some one'r de diseases de anermucls done brung "pun de people. An' de people got so dat dey knowed 'bout dese kyores, an' dat huccome dey have de medincin'-darnse.

"I done tol" you "twus a mighty big time. De medincin' men dey'd ga 'rr de yarbs an' git de medincin' raidy, an' den de leader he'd git up an' sing sump'n at de res' er de medincin' men, an' den dey'd 'spon' back at 'im wid some mo' singin'. Dey'd keep hit up dat-a-way a w'ile an' den de darnse wuz hilt. An' all de time hit wuz gwine on de people wuz drinkin' de medincin'. Hit wuz pass' roun' to 'em wid seven dippers in each mess er de medincin', an' er'y pusson 'bleeged ter teck some. Dey kep de darnse up fo' days, an' de fo'th day dey hatter go widout eatin' all day long. W'ensomurer dey have de medincin'-larnse dey alluz foller hit up wid de eagle-darnse an' de pigeon-darnse, an' each un 'em las' fo' days. In de eagle-darnse dey use de eagle-fedders an daddy say 'twuz a fine sight, all dem eaglefedders, each un 'em tip" off wid a li'l bit er fur f'um de deer's tail. Dem wuz gran' times, I tell you!

"Tell, I done tol' you dar wuz a lot er sickness an' de people wuz holdin' one $\mathrm{r}$ dem medincin'-darnses, an' ol Hrar" off up in de momntains see de smoke er de 


\section{AT TIIE BIG HOTSE}

fires an' come down ter jine in de fun. IT'en he fin' out 'bout de sickness, hit come inter his min' ter play doctah, so he go roun' sayin' he wuz a gre't medincin' man an 'axin' folks ter putt out der tongues. He’d say,

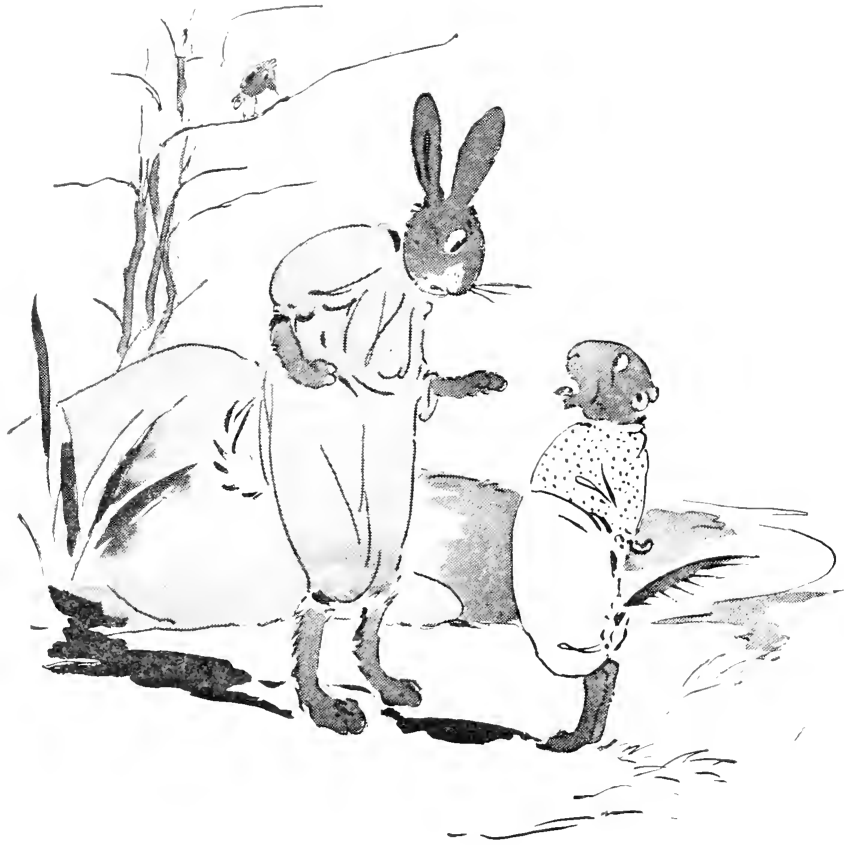

'Come yer, suh. an' putt out yo' tongue, fur 'z you kin, don' be 'shame' er de lenk ur hit, 'tain' ev'yb'dy got sech a good swollerer ez dat.' Den he'd come a li'l closter an' teck de tongue in his han' an' say, 'Sho! sho! yo' tongue mighty coated, suh. 'Pears lak yo' stummick kain't be wu'kkin' lak she orter; lessee 'bout dis.' Wid $10 \mathrm{~s}$ 
lat hed gin de tongue a snatch dat pull her clean out by de roots. He kilt two er thee pussons dat-a-way an' den tucken hisse 'f off an' hid in de big mountains whar dẹ kain't fin' him.

"De people dey" wuz plumb outdone, so dey hilt a krouncil an mek up der mins dey wuz gwine ketch 'im an' fix him so'st de fus' thing he knowed he oon know nuttin'. Dey say, De bes' way ter git "im down f'um de mountains be ter build a fire' so der all got toge ${ }^{\circ} \mathrm{r}^{\circ}$ an built a monst ous big fire. fus lightwood an' den bresh an' den logs. an' dey kep' pilin' her up an' pilin' her up 'twel you cu'd see de light fer miles. Dat fotched "im. But he wuz too smart an' too skittish not ter know dey wuz layin" fer "im, so he tucken his medjures cordin". He have de power ter turn hissef inter mos" anything he wanter, so he jes" tucken de shape ur a b'ar an' come scrunchin' "long an' squat down by de fire. De people wuz hid off in de bresh wid der bows an' arrers. but w'en dey see 'twan't nuttin' but a bar dey ses, "We ain' have no qu'oil wid Mistah Bar. Jes" we let him 'lone an save ow' arrers fer dat mis'able ol Hyar', so dey done dat. an' Hyar' he sot dar an' sot dar. sort er darin' em ter come on, but dey ain' spicion nuttin' an' atter w'ile he got tired an' mosered 'Iong back ter de mountains learin' 'em settin' dar watchin' an' lavin' fer “im.

"Dey kep' on buildin' fires an' he kep' on comin' an' settin' by 'em. fus' in one shape an' den in anu'rr 'twel las" de people gun ter see thu de dodge an' dey ses. 'Tell. suh, de fool-killer orter come 'long yer an' 'tend ter we-all. we sut'n'y has ac' lak plumb ijits, lettin' dat ol' rapskallion come yer night atter night an' set by de 


\section{A'T TIIE BIG IIOT'SE}

fire an' wo'm hisseff an' to lak he own de place. Te ain' sot no sense "t all er we'd a knowed twan't nob'dy else in de worl:. I boun' you he bin havin' a good laugh bout us wid de nabers w'en he got home. "Twon't do ter let dis run on no longer, fer of we does, he sho' ter git we-all inter hot water somehow er nu'rr. Now de on “ies way we kin mek sho' 'bout dis is jes' ter set back in de bresh an' let fly at any creetur whar come an' squat hissef by dat fire. We mus jes s'roun' de fire an' let de arrers zip him f"um er"y side.'

"Den dey went ter wu'k an' sont noration all thu de kyountry dat ev'y pusson whar kin hamnle a bow an' arrer mus come ter de meetin' dat night, eren de li’l boys wuz ter jine in. All dat day de men an' de boys wuz keepin' der han's in, shootin' at marks, an' de wimmins an' de chillen wuz bringin' de bresh an' de wood an' pilin' hit ligh fer de fire. Soon ez 'twuz dark dey lit 'er, an' my' how she blaze! I reckon you nurer seed no sech a fire ez dat wuz. Dar wan't no wind, an' de flame she jes' went a shootin' up in de air, an' ol' Hyar' he see hit, 'way off in de mountains, an' he rub his han's toge'rr an' he say, sezee, 'Nabers, I kain't stay home dis night, I tell you dat, p’in'-blank. Dars some kind er shindig gwine on down dar, by de lookin's er dat fire, an' I'se 'bleeged ter know w'at 'tis an' mebbe tek a han' in hit.'

"De nabers dey say, 'Yas, reckon 'twon't do fer you ter stay home one night, some 'mn might 'spicion dat you wuz a decent, sottled sort er pusson ef you done dat. Bes' g'long an' keep up de name you done mek fer yo'se'f.'

"Sezee ter hisse’f, 'Dog-my-cats ef I kyare.' an' wid 110 


\section{WHY THE TITAOCSE HAS A BLTNT TONGUE}

dat he riz up in his tracks an' putt out fer de low groun's so fas' dat he sca'cely tonch' groun' betwix' jumps.

"Jes' befo" he got dar he tuck an' change hirse"f inter nu'rr shape an' went rarin' an" chargin' up ter de fire lak he wuz pow'ful big an' empawtant. De people come mighty nigh bein tuck in by him ag"in, but de learler gin de signal an' dey close in on 'im, whoopin' an" yellin', an' ketched him jes" ez he wuz tryin' ter slip inter de bresh. No sooner is der tetch im dan he turnt back inter his own shape, an' dey ses, 'Th-huh, now we foun' you, suh, an' finders is keepers. you done yearn dat all yo' life. Neenter kick, suh, we ain' min' dat. To use ter thump de groun' wid yo' behime foot, neener, 'kase der ain' none er yo" folks widin call.'

"Irid dat dey drug him "way an" tied him to a saplin". Den dey all stood off an' tuck aim an' de arrers jes' come a-whizzin' thu de air, zippety-zip, an' struck ol' Iyar' mos' er'y time. He wuz too big a cunjerer ter be 'feard er dem li'l ol' arrers, so he jes' stood dar laughin'. II'en a arrer struck him hit 'ud jes' glance off widout hu'tin' him an' he do lak a flea bin an' bit him. Sezee, 'T'm-umph! is dat nu'rr flea done bit me? 'Pears lak I got a mighty big crap er fleas dis season, keep me pow'ful busy; 'scuse me, gemmen, ef I ain' ez mannerly ez wat I mos" in gin'ly js, dough I kin do dis an' lissen at you, too, at one an' de same time.'

"He wurk one eye at 'em an' wuz dat peart an' sassy dat de people fair' foam at de mouf an' dey say. 'Dish rers a sho'-'nuff cunjerer an' 'tain' no uze ow' tryin' ter kill 'im by owse'fs. Le's we send off ter one'r ow' medincin' men an' ax 'im w'at we bes' do ter kill dis owdacious creetur.' 


\section{AT THE BIG HOTSE}

"Dey kep" him tied up ter de tree an" sont off ter de medincin" man, an" he sar. "Co back an" tell "em ter shoot him in de haid." sio dey all tuck der bows an arrers an" aimed at de haid. Blesin goorhess, "twuz wuss dan befo". Hyar" jes" stood dar an' nurer even wonk his eyeleds ner twitched his whiskers, an' de arrers dey jes' fell off lak water f'um a duck's back. Dey seed 'twan't no use keepin dat up, so dey sont off ter de merlincin' man ağ in an' ax him fer ter please putt his min' ter wu'k an' he p 'em onter der fix, "kase ol' Hyar" an' daid yit ner showin' nair' sign er dyin', an' he 'bleeged ter git way f'm 'em an' do a lot er meanness yit of dey kain't kill 'im.

"De medinein' man study fer a w'ile an' den he say, "Well, you-all go back an' tell 'em I say dat de on'ies" way dey kin kill dat creetur is ter shoot him in de paw, dey kin try f"um now 'twel nurer an' dey kain't do hit no way but dat, an 'dey neenter try.'

"Dey went back an kyar'd de wu'd, an' all de men an 'bors got der bows an' arrers raidy ag"in an' de leader gin de w'u'd, 'Go!' Pey all p'inted der arrers at ol' man Hyar' an' let 'em fly toge'rr, an' Hyar' he knowed dat his time done come, but I tell you he died right game, sassy ter de re'y lis', axin' 'em w'y dey ain' think er doin' dat-a-way sooner.

"Atter he wuz daid an' dey see how easy 'twuz ter kill “im. dey git kind er mad 'kase dey ain' do hit sooner, an' dey ses. 'Now, w'y in de name er enmmon sense dir dat medincin' man putt us on de wrong track. tellin' us ter shoot ol Hyar' in de haid. w'y ain' he tol' us ter shoot him in de paw?'

"De mo' dey thought 'bout hit de madder dey got, 112 


\section{IHY THE TITIIOCSE IIAS A BLIXT TONGTE}

an' las' dey ses, 'Dat wuz a mighty ceitful, low-down trick, an seem lak we orter punish "im fer dat. "kase ef we lets hit go, he "ll git so we kain't putt no pennance on him it all an he ain he no kind er he p ter we-all.'

"De medincin' man he wuz neener mo' ner less dan de li'l bu id whar we calls de 'chick-a-dee.' De Injuns calls him 'Chick-a-lee-lee." w'ich soun' mighty nigh de same. Dey go ofl an' fin him settin' up on a branch. an' call him down an ax him wat do he mean by bein so ceitful an puttin' 'em on de wrong track. He ain' have nair' wu'd ter sar fer hisece dough mos' in gin'l he wuz right talky. He jes' hang his lil haid an' look at 'em outen de cornder ur his eye. I spec' der moughter kilt 'im ef he 'd gin 'em any sass, but 'kase he ac' lak he wuz saw' $y$ dey oon do dat. Dey ses, 'Chick-a-lee-lee, suh, you bin cunjerin' fer we-all dis long time an' we done place ow" pemnance on ro wu'd an' now you bin right 'ceitful wid us. 'Kase you done tol' us a fib we gwine teck an' cut off de cen 'ur yo tongue wat you tol' hit wid, an' dat gwine call hit ter ro' min' ev'y time you is temp'ed ter tell a fib.'

"So dey tuck an" tuck rhick-a-lee-lee an' cut off de tip een' ur his tongue an "Wat huccome Chick-a-lee-lees have blunt tongues uver sence lat time. An' uver sence den dey bin tryin ter curry faver wid de humans an mek 'em fergit wat dey done. Dey laks ter be near folks, an' dey mek derse fo mighty useful stroyin de bugs an' de w'ms offen de fruit trees. Even w'en de snow is on de groun' dey come hoppin' rom' de houses, an' dey look might ly please' ef rou tek notuss ur ' $\mathrm{cm}$. Dey sets der haids on one side an' looks up at you outer dey li'l bright eyes an' axes, 


\section{AT THE BIG HOtSE}

Chick-a-lee? Chick-a-lee?

Is you fren's wid me?

Ef you isn' you orter be!

Chich-a-lee-lee? Chich-a-lee-lee?

Chick-a-lee?'..

As she finished the story, Aunt Phrony looked around and saw her grandson stretched out on the ground behind her, fast asleep. "Wiyum ! Wi yum! Hi, Wi yum!" she called without effect. "Wake up, Wiyum! You year me. suh! Time I come dar an' walk up an' down yo" body a time er two, I reckon you wake up! I thought dat fotch you, suh. Jes' you g'long dis minnit an' ga'rr up dem nuts an' putt 'em in de bag, an' I 'spec' you-all chillen bes' he p me git de baskits an' things toge'rr an' go down ter de branch an' wash ro' face an' han's, 'kase yo' Uncle Hinry be plumb scannelize ef I brings you home lookin' lak dat. Den we mus' be morin' outen dis ef we gwine git home befo' sundown; dat is," as she saw signs of reluctance, "lessen you wanster stay yer all night long wid some er de creeturs I done tol' you 'bout." 


\section{THE WOMAN WHO MLARRIED AN OTL}

When the children got home from the nutting expedition and hat eaten supper. they sat around discontentedly. wishing every few minutes that their mother hat returned.

"I wish mamma would come back." said Ted. "I never know what to do in the evening when she isn't home."

"I 'low "bout de bes" you-all kin do is ter lemme putt you ter baid," said Aunt "Phrony.

"Don't want to go to bed." "I"m not sleepry" "Want to stay up," came in chorus from three pairs of lips.

"You chillen is wnser dan night owls," said the old woman. "Ef you keeps on wid dis settin"-up-all-night bizness, I boun some er you gwine turn inter one'r dese yer big. fury owls wid yaller eyes styarin. jes" de way li'l Nar Kit doin' dis re'y minnit. trin' ter keep hisse'f awake. An' dat 'mines me ur a owl whar turnt hisse'f inter a man, an' ef a owl kin do dat, w'ats ter himner one'r you-all turnin' inter a owl, I lak ter know? So you bes' come 'long up ter baid, an' ef you is right spry gettin' raidy, mebbe I'll whu'l in an' tell you 'bout dat owl."

The little procession mored upstairs, Coonie, the house-boy, bringing up the rear with an armful of sticks and some fat splinters of lightwood, which were soon 


\section{AT THE BIG HOT'SE}

blazing with an oily sputter. Connie scented a story, and his bullet pate was bent orer the fire an unnecessarily long time, as he blew raliant puff: upon the flames which no longer needed his asistance, and arranged and rearranged his skilfully piled sticks.

"Quit dat foolishmess, nigger," said 'Phrony at last, "an' set down on de ha"th an' 'have yo"se'f. Ef you wanter stay, whyn't you sesso. stidder blowin' yo'se'f black in de face? Now, den, ef y'all raidy, I gwine begin.

"Dish yer w'at I gwine tell happen at de time er de 'ear w'en de Injuns wuz havin' der green-cawn darnse, an' I reckon you-all bout ter ax me wat dat is, so I s'pose I mought ez well tell you. 'Long in Augus' w'en de Injuns stopped w'kkin de cawn, w'at we call 'layin' by de crap,' den dey cu'd mos' times tell ef 'twuz gwineter be a good crap. so dey 'mence ter' git raidy fer de darnse nigh a month befo han'. Dey went ter de medincin' man an' axed him fer ter 'pint de day. Den medincin' man he sont out runners ter tell er'b'dy, an' de runners der kyard 'membance-strings wid knots tied all 'long 'em. an' give 'em ter de people fer ter he'p 'em 'member. De foiks dey'd cut off' a knot f'um de string each day, an' w'en de las' one done cut off, den dey know de day fer de darnse wuz come. An' de medincin' man he sont out hunters, too, fer ter git game, an' mo' rumners fer ter kyar' hit ter de people so's't dey mought cook hit an' bring hit in.

"W'en de time come, de people ga'rred toge'rr an' de medincin' man he tucken some er de new cawn an' some ur all de craps an' burnt hit. befo' de people wuz 'lowed ter eat any. Atter de burnin', den he tucken 


\section{THE ITOMAN WHO MIARRED AN OWL}

a vear er cawn in one han" an' ax fer blesin's an' good eraps wid tat han", wile he raise up tu'r'r han' ter de storm an' de win' an ' le hail an’ baig 'em not ter bring evil pun de people. Atter dat, dey all made der

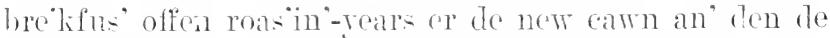
darnse begun an lasted fo days an fo nights; de men Aress' up in der bes" an' de gals wearin gre"t rattles tied on der knees. dat shuk an rattled wid er"y step.

"De gal whar I gwine tell bout wuz on her way home on de fo th night, an' she wru pow ful tired, "kase dem rattles is monstous hairy, an' she lin keepin hit up fo' nights han' rumnin'. She wuz gwine thu a dark place in de woods wen suddintly she seed a young man all wrop up in a sof' gray blankit an leanin "gins' a tree. His eves wuz big an' rom' an ' bright, an' der seemed ter bu n lak fire. Dem eves drord de gal an Arord de gal 'twel she wan't 'feard no mo", an' she enne nearer, an' las' he putt out his arms wrop up in de gray blanket an' drord her clost 'twol she loan erg'in him, an' she look up in de big. hright eyes an' the say, "Thar is you, whar is you?' An' he say, 'Oo-goo-coo, Oo-goo-coo.' Dat wuz de C'hurryee name for 'owl,' but de gal ain' pay no 'tention ter dat. for mos" er de Injun men wuz name' atter buids an" beasese an" sech ez dat. Atter dat she useter go out ter de wooks er"y night ter see de roung man, an' the alluz sing out ter him. "Thar is you, whar is you?" an he d arnser. 'Oo-goo-coo, Oo-goo-coo.' Dat wuz de on ies w'd he urer say. but de gal thought "twuz all right, fer she done mek up her min" dat he 'longed ter nu'r tribe er Injuns whar spoke diff"nt f"nm her own people. Sideren dat, she lore' him, an'w'en gals is in lore dey think ev'ything de man do is jes" 'bout 


\section{A'T TIIE BIG IIOLSE}

right, an dese yor cotin-couples is no gre`t han's fer talkin', nohow.

"De gal's daddy wuz daid an" her an" her mammy live all "lone, so las" she mek up her min' dat it be heap mo" handy ter have a man roun' de house, so she up an' tell her mammy dat she done got mäied. Her mammy say, 'You is, is you? Well, who de man?' De gal say 'Oo-goo-coo.' 'Tell, den,' sez her mammy, 'I reckon you bes' bring home dish yer Oo-goo-coo an' see ef we kain t mek him useful. A li'l good game, now an' den, 'ud suit my mouf right well. Te ain' have nair' pusson ter do no huntin' fer us sence yo' daddy died.'

" 'Mammy', sez de gal, 'I'se 'bleeged ter tell rou dat my husban "kain't speak ow' langwidge.'

" 'All de better,' sez her mammy, sez she. "Dar ain" gwine be no trouble 'bout dat, 'kase I kin do talkin' 'nuff fer two, an' I ain' want one dese rer back-talkin' son-in-laws, nohow."

"So de nex' night de gal went off an' comed back late wid de roung man. Her mammy ax him in an gin him a seat by de fire, an dar he sot all wrop up in his blankit, wid his haid turnt "way f"um de light, not sayin' nuttin' ter nob'dy. In de fire died domn an' de wind blewed mo'nful outside, an' dar he sot on an' on, an' w'en de wimmins went ter sleep, dar he wuz settin', still. But in de mawnin' wen dey woked up he wuz gone, an' dey ain' see hya'r ner hide ur “im all day.

"De nex' night he come erg" in and bringed a lot er game wid 'im, an' he putt dat down at de do' an' set hiscef down by de fire an' stay dar, same ez befo", not sayin' nair' wud. Dat kind er aggerrex de gal's mammy at las, 'kase she wuz one'r dese yer wimmins whar no 


\section{THE WOMAN WHO MARPIED AN OWL}

sooner gits w'at dey ax fer dan dey ain' kyare "bout hit no mo". She want son-in-law whar kaint talk, she git him. an den she want one whar kin arnser back. She gittin" kind er jubous "bout him. but she feared ter say anything fer fear he quit an she git no mo game.

"Thu'd night he come onct mo' wid a passel er game, an she mighty cur'ous bout him by dat time. She say ter huse"f, Thell! ef I ain got de curisomest son-in-law in dese diggin's. den I miss de queschin. I wunner wat mek him set wid his face turnt f"um de fire an blinkin" his eyes all de time: I wumer $w^{\circ}$ ' he ain nuver onloose dat blankit. an $w^{*} \mathrm{Y}$ he g"longs off 'fo' de daylight an' nurer comes back twel de dark."

“'Oh, mammy' sez de gal, sez she, 'ain' I tol' you he kain 't speak ow' langwidge an 'I 'spec' he done come f'um dat wo in krountry whar we year tell bout, 'way off ronner, an' dat huccome he hatter keep his blankit roun' him. I reckon he git so tired huntin' all day, no wumner he hatter blink his eyes ter keep em open.'

"But her mammy wan't sassified, kase hit mighty" hard ter haid off one'r dese yer pryin' wimmins. so she go outside an girr up some lightwood splinters an' thow em on de fire. lis-awar, all ur a suddint." Here the old woman rose and threw on a handful of lightwood, which blazed up with a great sputtering, and in the strong light she stood before the fire enacting the part of the scared $\mathrm{OW}$ for the delighted yet half-startled children.

". In' w'en she thowed hit on," Aunt 'Phrony proceeded. “de fire blaze an" spit an' sputter jes' lak dis do, an' de ooman she fotched a yell an' cried out. she did, 'Lan' er de mussiful: W'at cur'ous sort er rood is dish 


\section{AT THE BIG IIOLSE}

yer dat ae' lak dis?' De Owl he wnz startle' an' he look rom" suddint, dis-a-way, orer his shoulder, an' de wimmins dey let out a turr"ble sereech. "kase dey seed twan't nuttin' but a big owl settin' dar blinkin'.

"Owl seed he wuz foun' out, an' he riz up an' give his gre`t. wide wings a big flop, lak dis, an' swoop out de do" cryin' Oo-goo-coo! Oo-goo-coo!' ez he flewed off inter de darkness." Here Aunt "Phrony spread her arms like wings and made a swoop half-way across the room to the bedside of the startled children. "An"," she continued, "de wind how mo'nful all night long, an' seem ter de gal an her mammy lak "twuz de voice of po" Oo-goo-coo monin fer de gal he love."

"And didn"t he ever come back?" said Ned.

"Naw, suh, dat he didn'. He wuz too 'shame' ter come back, an' he bin so 'shame' er de trick wrer sence dat he hide hisse $f$ way in de daytime an" nuver come out "twel de dusk, an' den he go sweepin" an" swoopin" "long on dem gre"t big sof" wings, so quiet dat he ain" mek de ghos' ur a soun', jes' looks lak a big shadder flittin' roun' in de dusk. He teck dat time, too, 'kase he know dat 'bout den de li'l fiel' mouses an' sech ez dat comes out an' 'mences ter rum roun', an' woe be unter 'em ef dey meets up wid Mistah Owl; deys a-goner, sho'."

"But how could they think an owl was a man?" asked Janey.

"Well, honey, de tale ain" tell dat, but I done study" hit out dis-a-way, dat mo'n likely de gal bin turnin' up her nose at some young Injun man, an' outer spite he done gone an' got some witch ter putt a spell on her so's't de Owl 'ud look lak a man an' she 'ud go an' th'ow husse'f away on a ol no-kyount bu'd. Yas, I 
THE WOMAN WHO MARRIED AN OWL

reckon dat wu 'bout de way. In' now y'all better shet up dem peepers er you'll be gittin' lak de owls, no good in the daytime, an' wantin' ter be up an' prowlin' all night." 


\section{MR. IILD-TURTLE'S ADVENTURE}

The next day the children went down to the river for the first time, under the guidance and protection of William, who knew the short cut throngh the lowgrounds and just which were the places where rou were most likely to get a bite, for the rirer and he were old and dear acquaintances and had commmed together through many a long. sumny day. The river had imparted many of its choicest secrets to him, for there is no one with whom Madam Nature lives on closer terms of intimacy and sympathy than with that wholly untrammeled and entirely natural animal, known as "The Boy."

William was the possessor of a rast store of information dear to the heart of childhood, and was never better pleased than when called upon to impart some of his knowledge to the less enlightened. He knew and could imitate the notes of the birds and the cries of the beasts. He could tell when spring was approaching or summer departing by the arrival or departure of certain birds, or the preparations made by certain animals. He conld tell time by looking at the sun and was learned in weather-signs. He had other information, besides, of a lesser but more practical sort, for he knew the whereabouts of a shellbark hickory, the only one for miles around, and had located a persimmon tree whose crop 


\section{IIR. IIUD-TURTLE:S ADTENTIRE}

never failed and whose fruit was described as "monst ous" for size. Ile knew how to make a running noose, and could lasso a fleeing calf with the ease born of long practice. He was an authority on jack-knives, and could carve peach-stone baskets, make slings and whisthes and manufacture bows and arrows. He was well up in marbles and knew just how many "commies" to give for an "ally," and how many allies for a "glassy," and if he played "for keeps" was pretty sure to do all the keeping.

"Jack-straws" and "mumble-the-peg" were also among his lighter acomplishments, but the one which exalted him most in the eres of the children was his skill as a barcback rider. As he rode a big horse to water, sitting sidewise, whooping and digging his naked feet into the animal to urge him to greater speed, their admiration was unbounded. Tet this was not the end of his resources, for he could take you to a wonderful graperine swing. in which you could swing perilously out over the edge of a deep hollow, holding your breath fearfully until you landed on teria firma again; or he could pilot you to an old peach-tree which exuded enough gum to supply a dozen children; or he could take you to a place where Indian arrow-heads were still to be found: and added to all this, he knew a spot in the garden where the youthful fishermen could always find worms for bait.

He was important and happy as he marshaled his little company down to the bank of the dark, sluggish river that. like some noiselessly gliding snake. wound in and out beneath the eypresses and the pines. He chose a safe spot for the children, near a shallow, and baited 


\section{AT THE BIG HOLSE}

hooks all around, and then, removing himself a short distance, sat immorable, pole in hand and bait in mouth, waiting for a bite with all the patient impatience of the born angler.

His little companions found too much to look at and wonder about and talk over to make a success at fishing, and they sadly interfered with his sport; but after a while they tired of waiting for a bite and wandered up and down the bank in search of shells and bright pebbles and all the other treasures which generous rivers lay out upon their banks within reach of small hands. Then they wandered a little way into the bushes in search of woodland treasures, and at last, growing tired, they rested beneath a tree, while little Kit laid his head in Janey's lap and fell fast asleep. Here Aunt 'Phrony found them when she came to say that dinner was ready.

"Whar dat boy Wi"yum?" she asked; "fishin' yit, I'll be boun '! Jes' let him git a pole in his han' an' he nurer know ef he's 'wake er sleep er full er empty. Wi'yum! You Ti'yum! Kain't you arnser me, er is I gotter come dar an' stomp all orer you!".

Howerer, she was not forced to administer correction in that comprehensive form, for just then Wi'yum gave an excited whoop, as though to announce something important, and on hurrying down they found him in the act of securing a good-sized mud-turtle. Aunt Phrony lent her aid, in as great a state of excitement as Wiyum himself, whom she adrised to be very careful or he might lose his turtle, for "mud-turkles," she said, were "mighty" slickry an' onsut'n, an' lessen you keeps a sharp eye 


\section{IIR. MLD-TLRTLE:S ADTENTLRE}

on "em dey may git "way f"um you. same ez dat trickish ol Mristah Mud-turkle git "way fum de creeturs one time w"en dey thought dey sho"'nuff had "im. Ef y'all hurry up yo cakes an ga'rr yo trash toge ${ }^{\circ}$ 'r an come long home dis re'y minnit, mebbe I'll tell you dat tale ez we go "long:"

The treasures so disrespectfully referred to as "trash" were caught up in great haste and the homeward procession formed. Wi yum leading the way, proudly bearing the mud-turtle.

"Well, ez I wuz sayin"" the old woman went on. "Mistah Mrud-turkle he wuz a mighty slickry man. an" he bin up ter mo tricks dan you kin shake a stick at, an" he git right onpop "lous wid de creeturs. He knowed dẹ wuz sort er layin" for "im. an" er"ry now an" den he"d come up f"um de bottom er de rirer an ketch 'em on de bank watchin" fer "im. Den he dick his behime laigs up in de air at 'em an' dive down wid a gre't splash. Atter a li"l he"d come up ag"in. mner de shadder ur a big lily pad. an' lay jes" below de top er de water wid his snout out ter git de air. watchin" de creeturs whar wuz layin' fer him. I"en dey wan't lookin' hed climb up on de log ag"in. an" ef dey showed signs er mekin' a snatch at "im, down hed go wid anu rre splash.

"One time dey ketched "im w"en he bin mo' rantankerous dan urer befo". an" dey ses to "im. "Mistah Mudturkle, we is re sa $w^{\circ}$ we hatter speak so plain ter you, but de trufe is we done gin you a long rope an' now you is come ter de plumb een" ur hit an we kain't pay out no mo". we "bleeged ter pull rou up shawt on de road you is gwine, an' we'se sut'n'y saw'y ter fotch you 


\section{AT TIIE BIG IIOCSE}

up wid a ju'k but we done made up ow' min's dat we mus do lit emejiately, so we gwine exescoot you on de spot.'

“Ol' Mud-turkle he 'suade an' he 'suade an' he 'suade, but 'twan't no use, an' las' he say, 'Gemmen, dish yer is mighty hard on bofe un us, 'kase I 'low dat you ain' hone ter do dis an' I knows I ain' hone ter have hit done, but ef exescoot is de w'ud, wy den exescoot mus' be de deed. But I ax y'all, gemmen, 'kase you is fambly men, same ez me, ter please lemme go home an' bid far'well ter my ol' ooman an' de chillens.'

"Den he drord down one er his th"ee pairs er eyeleds an' sot dar lookin' jes' ez sleepy ez rou please an' ez harmless ez ef he ain' nuver kotched an' et even so much ez a frog er a tadpole.

"De creeturs wuz might"ly tickled by w'at he ax 'bout goin' home ter bid far'well, but dey wan't tucken in by hit, so dey ses, 'Saw'y not ter 'commodate you, but today's right yer an' to-morrer's right dar, an' de bu'd in de han' 's wuf two in de bush, so you mought ez well mek yo' reddymints an' say yo' pra'rs, 'kase we is gwine putt you in de midse er dish yer big fire we buildin' up, an' dat 'll mek sho' you kain't cut no mo' er yo' highfalutins in dese diggin's.'

"Turkle he wuz kind er cas' down fer a minnit, an' sot dar wid his jaw drapt, but pres'n'y he git a notion dat perk him up might'ly, an' he say, 'Sho! you-all done tucken so much trouble 'bout dat fire dat $I$ is mos' 'shame' ter tell you, yo' wu'k ain' gwine 'mount ter shucks, 'kase I wuz bawnded an' brunged up in de fire. an' ef dar is one place mo'n a nu'rr whar I feels at home, hits spang in de fire.' 


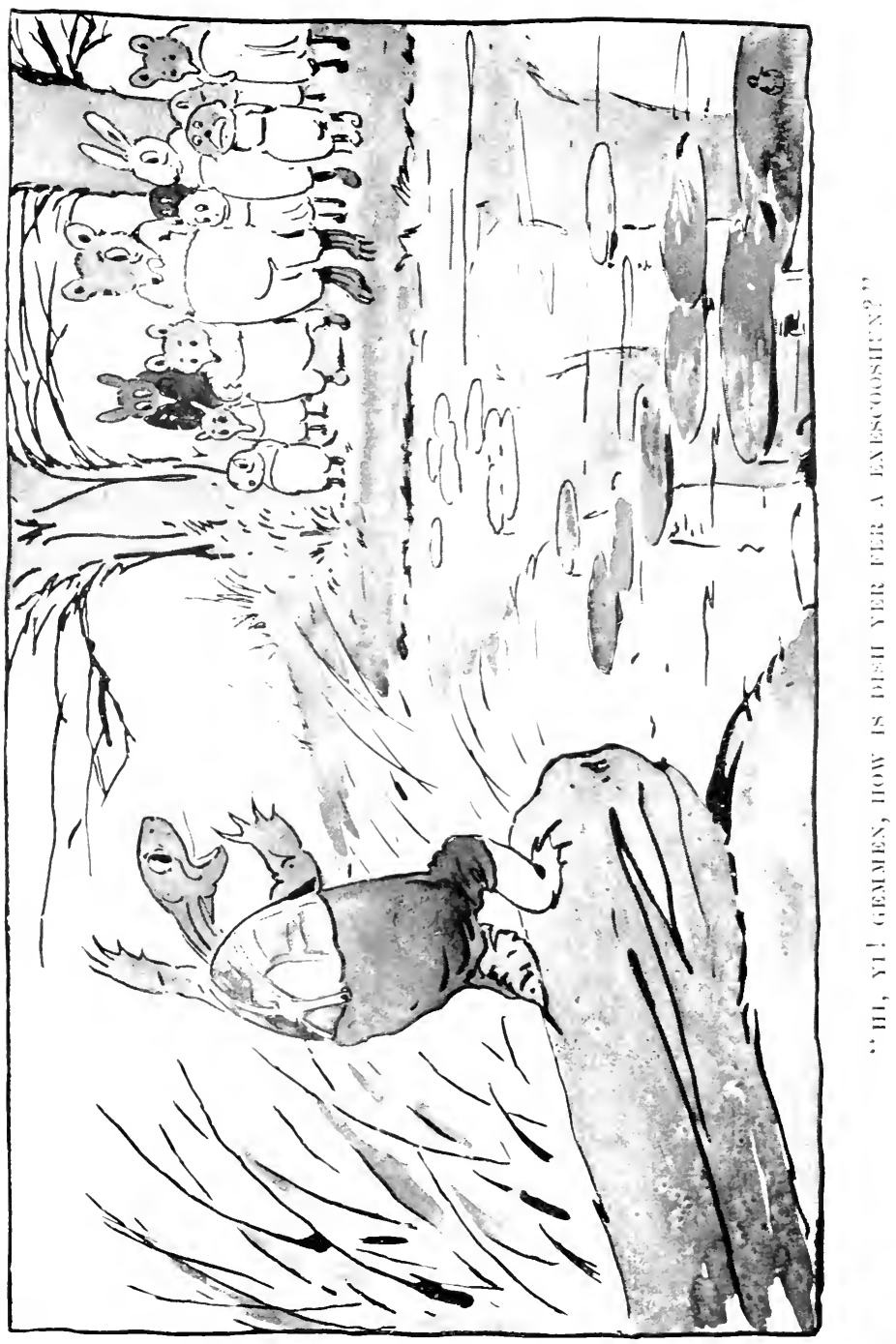





\section{MR. IILD-TTRTLE:' ADVENTLRE}

"Den de creeturs go off lil ways an' have a confab, an' den dey come back an" putt a big pot er water on de fire, an' w'en hit git ter bilin', dey ses. 'Well, Mistah Mrud-turkle, yo' time is sho'ly come now, fer onct you strike dat bilin' water we gwine th'ow you in, hit 'll be good-by, Mistah Mud-turkle.'

"Den he say, sezee. 'Gemmen, I'se saw'y ter see you was"e so much er yo" time on a no-kyount creetur lak me, so I gwine tell you you kain't kill me dat-a-way. I ain' min' ef you putt me in de pot. 'kase all I gotter do be ter scrabble roun' a li'l an' kick out my footses, an' I got sech strenk in 'em dat firs' news you know de pot be over an' mo in likely some er you-all whar ain' got a shell on you be scalt stidder me. I'se saw'y ter upsot ro' plans, but I be boun' ter upsot de pot, dat's de gospel trufe.'

"By dat time de creeturs wuz kind er outdone, an' dey say. 'Le's we-all tek de mis'able ol' bag-er-shucks ar' thow him ker-mack inter de river. I boun' you dat be big 'nuff so'st he kain't kick outen hit.'

'Ol' Turkle he snicker ter hissef w'en he year dat. 'kase he see dey done fergot he b'long in de river. Den he say wid a turr"ble whine in his roice, 'Oh, please, gemmen, fer de love er mussy don' you go fer ter thow me in de river. Kill me any way you wanter, but not dat; seem lak I kain't stan' hit ter be at de bottom er de col', rlark river.'

"He kep' gwine on dat-a-way kase he knowed de creeturs be sho' ter putt him dar ef dey think he ain wanter go, but in cose de river wuz prezackly de place whar he b'long an' whar he wanter be.

"Sho' 'nuff! dey tucken him by de tail an' drug him 


\section{AT THE BIG HOLSE}

down to de river, him baiggin' all de time. 'Oh, please, suh. lemme go! Please don" do me dis-a-way! Lemme off dis time an ' I ain' do so no mo"!' De creeturs ain' pay no 'tention but go right on an th'ow him inter de deepes' place whar dey kin fin". W'en he git ter de bottom he laid dar still ez a mouse, not eren so much ez twitchin' his tail, an' dey thought he wuz sho"-nuff daid an' went off an' lef" him.

" $\Pi$ " en dey git off a piece, he open one eye mighty krarful an' wall hit roun' a li"l an' seed de coas' wuz clèr. Den he "gun ter climb out on tu'rr bank. W'en he wuz clean up on de bank an" knowed dey cu'dden git "cross to him. he fetched a big whoop, an" w"en dey turn roun' ter look he sing out. 'Hi. yi! gemmen, how is dish rer fer a exescooshun? Come "long orer yer an' exescont me some mo"! I'se feelin' tol'ble lively fer a cawpse, I is!" An' wid dat he fotched anu'rr whoop an' dove down de bank inter water. haid fo "most. an' lef" 'em dar fair foamin' at de mouf. An' I reckon we better be hurrvin' 'long li"l faster'n we has bin, er 'Liza she be foamin' at de mouf. too. 'kase you done kep' de dinner waitin'. Cooks is mighty tetchy 'bout dat." 


\section{WHY THE FLOCNDER IS FLAT}

The children's mother returned the next morning, with Aunt Nancy, and the latter greeted her little charges with much hugging and many warm words of affection. "Come yer, my honey-buds, my sugar lumps, my apple-dumplin's, come yer ter yo' ol' Aunt Nancy an' gimme a good, tight hug! Bless my soul, dese yer de sweetes' things I seed sence I bin gone. Bless dis baby's heart, I ain' seed no sech yaller curls ez dem, dat I ain"! Gimme nu'rr hug! Um-umph! I dunno w'at de ol' ooman gwine do w'en dese chillen go off an' leave her. 'Spec' she hatter foller 'long wid 'em. Come yer ag in an' lemme see ef dat 'Phrony bin keepin' y'all good an' clean."

All day she kept them on their best behavior by telling them that she had heard two new stories while she was away, which she proposed to tell them that evening if they had been good enough to deserve it.

That night when she was putting them to bed they inquired anxionsly if their conduct had been all that she could desire, and if the stories were to be forthcoming. Janey, by way of a clincher, informed her that Aunt 'Phrony had been telling them stories every night, "and sometimes in the dar-time, too."

Aunt Nancy appeared to pay no attention to this, but it nevertheless had the desired effect of hastening the story-telling. 


\section{AT TIIE BIG HOTSE}

"Dese tales," she said, "I yearn f"um a tide-water nigger: one whar live in ol (Bloucester, by de salt water, all his life. Tat hnccome one'r de tales is 'bout fishes an' mo special $w^{\circ} y$ de flounder is flat. You know mos' er de fishes jes stan's up on der narrer aidges an cuts thu de water dat-a-way, but de flounders dey lays flat 'cross de water an' goes flounderin' 'long, so-fathion. Dey sut'n'y is curisome creeturs, 'cordin' ter wat dat man tol' me. He say dar haids look jes' lak dey wuz all wrench' roun' ter one side, wid de two eyes growin' on one side, stidder bofe, de way 'tis wid mos' fishes, an' der moufs look lak dey growed crooked, too. An' stidder bein' de same color on bofe sides, dey"re white beneaf an' dark on top, jes" de color er de mud. banks dey lars on at de bottom er de water. Bein' ez dey lays low dat-a-way dey ain hatter watch out beneaf 'em, jes" hatter keep on de lookout fer sump'n up abore 'em, an' so dem eyes on de top side jes' suit "em prezackly.

"Well, dat Gloucester man he say dat onct dar wuz noration sont out thu all de rivers an' de Gre't Baydat mean de Chisapeake-dat de fishes mus' meet toge "rr an' choose 'em a king whar wuz ter be King of all de Fishes. De big fish ac' mighty uppitty w'en dey year dis. Dey snort an flap der tails an' jump outen de water an' say: 'Tmph! leare dat ter de small fry, we-all is kings a raidy. Folks dat kin do ez dey please, an' go whar dey please an' ketch an' eat up de li'l fish w'en dey please, an' ain' kyare whe'rr de wind blow er de sun shine, an' kin swim slow er swim fas' an' ride on de big wares out inter de deep waters; w'y, dem folks is sho'-'nuff kings an' ain' got no use fer jes' de name 


\section{WHY THE FLOCNDER IS FLAT}

uv hit. 'Deed dem is de on “ies lings w'at dey is: tu'rr kin' is jest de sarvants er de people. 'Scuse we-all f"um sech ez dat. Let de small fry choose a king fer deyse'fs, of dey wanster. I boun' you dis marter gwine stir up a big mess 'mongs' 'em an' keep 'em 'sputin' fer a Jnont' er Sundays.'

"So de small fry met toge'rr, down ter de las' li'l minner, an' dey comed f'um fur an' f"um near, f"um ery lill river dat run inter de Gre`t Bay, an' f'um er"y part er de bay hitse'f, an' f'nm de deep water an' f'um de shaller, an` dey comed roundin' de p "int in shoals an’ shoals, so many dat you cu'd scacely see de water fer de fishes, an' dey sailed on up inter Mol Jack Bay whar de meetin' wuz ter be. I tell you dat mus' 'a bin a gre’t sight. An' ez dey swim 'long der fins made sech a noise cuttin' thu de water, 'kase dar wuz so many ur 'em, dat hit soun' lak de sides ur a monst ous big ship cuttin' an' swishin' thu de water.

"W'en dey wuz all ga'rrd toge'rr you nuver year no sech a splashin' an dashin' in all yo" bawn days. Dey dart yer an' dar an' back an' fo 'th an' up an' down, an' dey talk an' dey talk an' dey talk, but dey kain't seem ter mek up der min's, 'kase de trufe wuz dat ev'y las' man er de lot thought he orter be king. Thee er fo "ud putt der haids toge'rr an' whusper an' say, 'Mell, suh. who you think gwine be made de king?' "Who is yo' chyce fer de place?' 'Now what you think 'bout dat feller so-an'so? I kin see he's atter de job, dat's plain ez a pikestaff, but I bin knowin' him urer sence he wuz a minner; 'deed we wuz bawnded an' brunged up in de same shaller, an' I kain't see nuttin' to 'im. I be boun' ef I gwine gin my rote ter no sech a numbskull ez dat.' 


\section{AT THE BIG IIOLSE}

"W"en dey ax one nu'rr "Who is yo" chyce?" each un 'em 'spec' ter year tu'r say. 'Mry you, suh, you is de ve y man ter suit de place.' But each fish want de place fer hissef, so dey jes' shake der haids an' say: 'Dumno! dumno nuttin' 't all 'bout hit. Dese things mighty hard ter fin' out. You nuver lin tell who gwine come out on top.'

"Las,' li'l White Perch git kind er tired foolin' roun' so long. an' he tell 'em he gotter be swimmin' 'long to des home, fer he live over on de Eastern Sho' an' mus git dar fo" dark, "kase he say dar wuz er"y chanct in de worl' dat he git nipped up by some er de big fish ef he travel atter sundown. So he 'low: 'Gemmen, I'se a numble an' onignoran' man an’ may irrepresent mysef. but sence none er you speak ont in de meetin: I gwine do hit myse f. I name to you my fren Mistah Billy-fish. He de bes man I knows fer ter teck dis place whar seem ter be gwine baiggin' 'mongs' y'all. Le's we mek him king an' gin him de name er King Billy.'

"Dar wuz gre"t 'miration all thu de meetin' orer dat talk er White Perch. Sech a whusperin' an" confabbin' an' haid-shakin', you nuver see de beat: an' dey waggled der fins an' der tails an' blowed water thu der gills 'twel Mob Jack Bay wuz all ur a foam. But dey wuz all so sot on bein' king dat nob'dy speak up an' name any urr fish. Den dey kep' still a w'ile an' thought over de marter. "kase dey hate might'ly ter gin up de notion er bein' king derse'fs, but dey seed de time wuz gwine by an' nuttin' done, so dey 'gree at las' an' mek Billy-fish king, an' all crowd roun' an' pass de time er 


\section{WHY TIHE FLOTNDER IS FLAT}

day wid 'im an' call 'im 'King Billy.' An' he wuz de king in dem waters fer 'ears an' 'ear's, an' he have a big fambly er chillen, an' ter dis day you lin fin' lots er his folks in de Gre`t Bay an' in all de li'l hays an' rivers.

"De Flounder done comed ter de meetin' feelin' sho' de people gwine teck him fer king, an' w'en he year 'em callin' Billy-fish 'King Billy', he wuz dat mad he turnt raid in de gills. 'Mis able li’l Billy-fish!' sezee; 'ter think er dat creetur bein' made king w'en he dunno 'nuff ter teck good krare ur hisse'f, let 'lone lookin' atter urr folks. In' ver me whar know mo'n he uver gwine fergit, an' dey done pass me over same'z I ain' bin yer. I ain' gwine be boss' by no lil ol' no-kyount billy-fish; now you year me talkin"! Not much bigger'n a minner! Him ter have de say er me? Naw, suh, not w'ile my name's Floppy Flounder!'

"Wid dat he gin his haid a toss ter one side an' puffed out his gills an' said 'Sho-o-o!' an' lo beholst you, he gin his haid sech a pow'ful ju“k dat hit jes' stayed ter one side an' he ain' nuver bin able ter git hit back ag'in; so he hatter go swimmin' 'long wid de flat side up.

"IV'en tu'rr fish see dat, dey think he jes' doin' hit 'kase he mad, an' dey ses ter one nu'rr: 'Ili! ol' Floppy Flounder gittin' mighty biggity, gwine rom' rer wid his haid all raared ter one side, jes' 'kase we-all ain' mek him king. I boun' his ol' neck git tired an' he be glad ter turn hit back 'fo' long.'

"But pres'n'y dey fin' out he kain't git hit back nohows an' den dey felt saw'y fer him an' tried ter he'p him turn hit 'roun'. but 'twan't no use, she'z dar ter stay; an' so de po' creetur bin gwine rom' dat way urer 


\section{¿T TIIE BIG HOLSE}

sence, an' people year ur him fur an' wide, an'w'en dey wanter say anything's flat dey say hit's 'flat ez a flomnter.'

"Pears ter me," the old woman added, with a meaning glance at Janey, "pears ter me I done seed a li'l gal tossin' her haid at her mammy dis ve'y day, an' I ses ter mrefe. den an' dar, dat I gwine tell her de tale er de Flounder. so sth she know monght happen ef she keep dat up. Chillen's haids ain'stuck on no closeter dan fishes' haids, I reckon; so dey bes' be kyarful w'at dey does wid 'em." 


\section{BROTHER SQLTRREL AND MOLLY HARE}

"Now, den," said Aunt Nancy, when she had finisher the story of the Flounder, "I reckon y'all done had 'nuff fer dis night, imn' you?"

"No, we havent." said Ted, with an injured air. "You said you'd tell two to-night if we were good; and so I didn't do lots of things I wanted to, and I was careful about getting over the fences and only snagged my trousers once, just a teenty little hole."

"And I only took one piece of pie for dinner; I said 'No, thank you,' when they offered me another, but I wanted it all the time," said Janey, "and there were lots of things I could have done if I hadn't been trying to behave, and I"ll go and do "em to-morrow if you don"t do what you said you would."

The old woman looked at Janey over her huge, brassrimmed spectacles with as severe an expression as her good-natured face could assume.

"Well, I ain' de one gwine be hurt by yo' nortiness," she said, "but of I done promuss you two tales, den I gwine gin you two, 'kase I ain' gwine back on my wu'd, even w'en chillen ac's kind er uppish wid me an' gits ter talkin' onmannerly. Dis tale is 'bout Brer squ'l an' Mis' Molly Hyar', an' hit wuz telled ter me by de same man whar tell me 'bout de Flounder an' tu'rr fishes. 


\section{AT THE BIG HOTSE}

"He say dat in de ol", ol times. MIis" Molly Hyar" useter wunner w' "twuz w"en her an" Brer Squ'l look so much lak one nu'rr, dat he live up in a tree an' she live down on de groun'. 'I"se er'y bit ez good ez he is.' she say ter husse'f. 'I 'spose he feel mighty biggitty 'kase he live up dar in de tree. an' I reckon he kind er look down on groun'walkers lak me. But I boun' you I gwine teck him down a peg er two nex' time I see "im. Mis'able li'l skeezicks! So proud er dat bushy tail dat he all time rarin hit up clean over his haid so"st folks kin see hit.' She talk dat-a-way "kase she right tetchy "bout her own tail whar got nipt off shawt one"r dem times w'en she wuz up ter some ur her "harishness.

"Las', one dar, she met up wid "im at de foot ur a tree. He wuz settin' up gnorrin' a nut an" turnin" hit roun' an' roun' in his li'l pars. She stop' an squat down on her ha'nches an' pass de time er day wid "im. 'Howdy, Brer Squ'l,' she say, an' he stop' gnorrin' an' hilt de nut in one li"l paw an "spon', "Howdy, ma'am. howdy: "kase he wuz a mighty mannerly li'l feller an' on go on eatin" w"iles dar wuz a lady roun'.

"Den, he say: 'Mis" Molly. won't you please, ma'am, come up ter my house in de tree an' mek yo"se"f comf"ble an' at home? I be proud ter see you in my house, ma'am.'

"Naw. thanky, suh." sez she: 'I come a right fur ways to-day, an' I ain feel ekal ter de climbin': ain" ez young ez I onct wuz, Brer squ'l.' She talk dat-a-way 'kase she ain' wanter let on dat she wan't use ter climbin' trees.

"Pres'n'y she say, sort er sniffin' an' turnin' up her nose all de time she talkin": 'Brer Squ'l, you an' me 


\section{BROTHER SQLIRREL AND MOLLY HARE}

sut'n'y look sump'n lak one nu'rr, but seem ter me hit's on 'y skin deep: we ain 'ac' nuttin' 't all de same. You eat: nuts an' I eats grardin-truck; you lives in a hole in de tree. an I lives in a hole in de gromn'; an' I reckon I kin do a heap er things you kain't do. 'Pears ter me 'bout de on 'ies' thing you kin do is ter run up a tree.

"Brer Squ"l he drap de nut an' hang bofe paws, kind a' meek an' numble. an' he say, sezee: 'Fur be hit f'um me ter say dat I is de ekal ur a nice lady lak Mis' Molly Hrar". But please. ma am, ter show me some er de things you kin do. kase I done year tell dat you is a monst ous spry ooman.

"Molly she snigger at dat an' say: 'G'long 'way f'um rer, Brer squ'l. w'at you wanter bamboozle a po' ooman fer. wid ro' flattersome wuds? You men-folks orter be 'shame' er ro"se"f: 'deed you ort.'

"Squ"l he say, sezee: "Tellum, you kin b"lieve hit er not, but I alluz means w'at I sez; an' de nabers'll all tell you de same. I wuz bawnded yer, las' 'ear, an' brung up right in dis tree, an' dey all knows me right well.'

“' Trell, ef you talkin' de trufe,' sez she, sniggerin' ag in lak she still feel kind er backwu'ds 'bout hit, dough all de time she wuz jes' a-honin' ter show husse'f off; "well, ef dat de trufe, den I gwine whu'l in an' show you some tricks whar is tricks slo'-nuff: hit teks me ter do 'em.' Wid dat she turnt husse'f aloose, an' uv all de gwines-on you urer see, dem tuck de lead. She go kitin' long a piece. an' den all ur a suddint she r'ar up on her ha'nches an' do lak she lissen: den she gin a monst'ous jump an' go on, an' den she hide, an' 


\section{AT TIIE BIG HOLSE}

den come out ag“in: an' den she double lak de dogs atter 'er an' she wanter git 'way f'um 'em. an' she dat spry an' soople seem lak she do all dem tricks widout half trvin".

"Brer Squ'l he monst"ous p"lite, an" he watch her clost an' pay good 'tention an' wuz mighty please' wid ev'ything. He clap his li'l paws an' say: 'Good fer you, Mis" Molly Hrar"! You sutn'y is a sho"-nuff jumper! I boun dar ain' nair' $d o g$ in dese parts kin run you down, Mis' Molly.'

"She say: 'Oh shucks! dis ain' nuttin' 'tall. Wait 'twel I show you wat's w'at.' An' wid dat she 'mence all over ag"in an putt in all de extry licks she cu'd think ur, scootin' in an' out 'mongs' de trees an' de bushes so fas' dat rou cuidden see no laigs ner feet. jes' look lak a wad er fur dat wuz bein' chunked roun' fer fun.

"Las', Squ'l he 'feard she gwine tire husse'f all out. 'kase he wuz a kin'-hearted li'l feller, an' he say, sezee, 'Thanky. Mis' Molly, thanky, ma'am. I much 'bleeged ter you fer showin' me yo' tricks, an' I owns up dat I kain't do one un 'em. But mebbe, ma'am, you lak ter see me run up a tree: fer you done tol' de trufe w'en you ses dat's 'bout all I kin do.'

"But, bless yo' soul, he kin do heap mo'n dat, on'y he wuz one'r dese yer modes' mens whar thinks mo' ur urr folks' doin's dan dey does ur der own, but I let you know dat lots er times dey kin do mo' dan de peoples whar meks sech 'miration orer derse'fs.

"He kite up de tall tree same`z ef he wuz rumnin' on de groun', an' w'en he git dar he do all sawts er things. He pick nuts an' swing back an' fo th on de een' ur a limb, an' jump f'um one tree to anu'rr widout losin' 


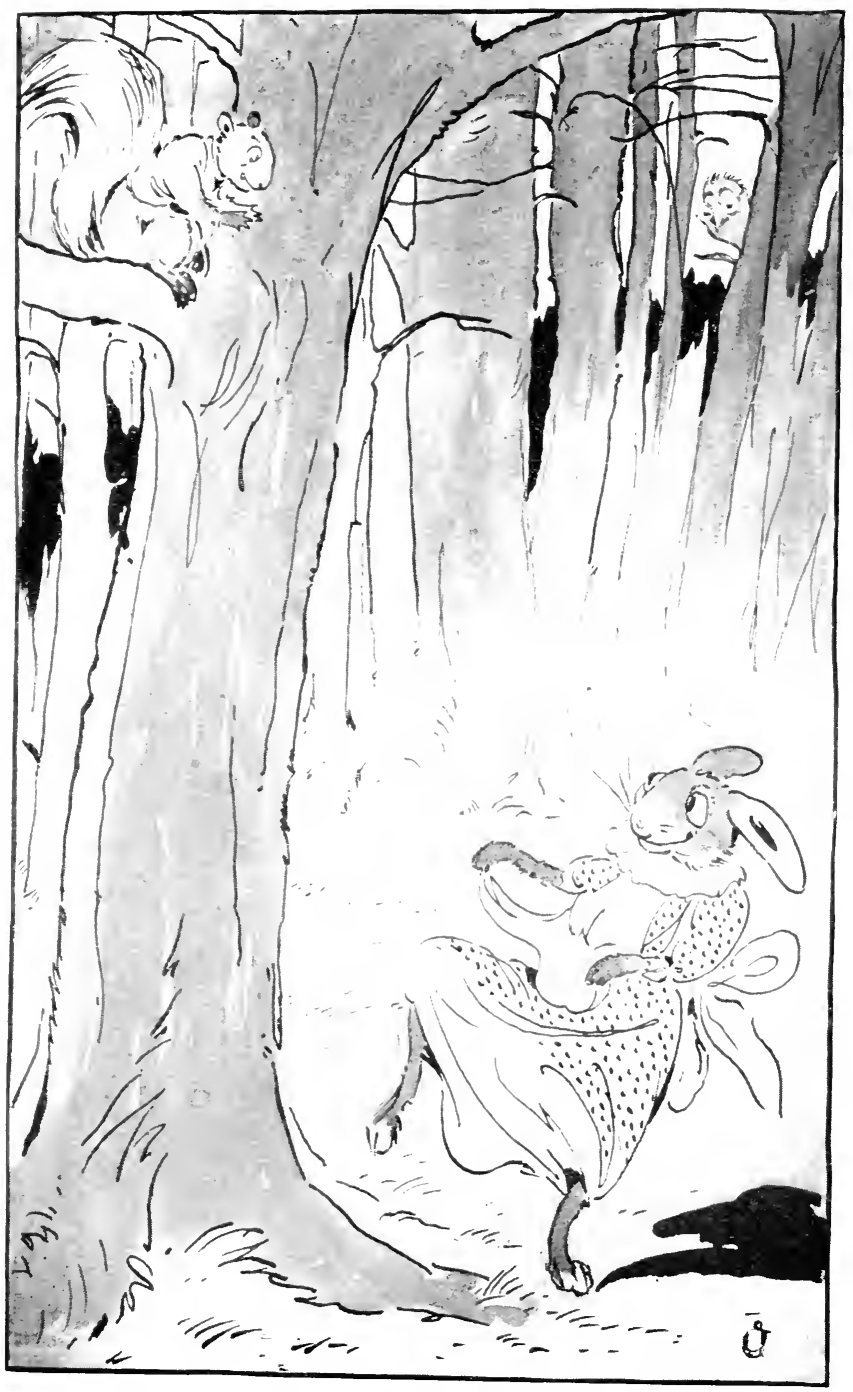

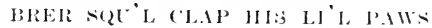





\section{BROTHER SQUIRREL AND MIOLLY IIARE}

his baluns er fallin' onct. Den he spread out his fo' laigs wide ez he kin an' jump f'um de highes' limb clean to the groun' widout hurtin' him 't all, an' wuz up de tree ag"in befo' Mis' Molly cu'd wink her eyeleds. Tex', he run down de tree haid fo'most, jes' ez easy ez he run up. Den back ag'in ter swing some mo' an' hang on by one paw an' turn a somerset on de limb, an' a hull passel ur urr tricks.

"Mis' Hyar" she wan't so pleasan' an' p'lite ez Brer Squ'l bin. She ain' say nuttin' nice 'bout his tricks, jes' set down dar below, squatulatin' wid 'im an' rollin' her eyes up an' wringin' her paws an' sayin' over an' over ag“in: 'Brer Squ'l don' min', he kill hisse'f! Brer Squ'l don' min', the kill hisse'f! Gwine fall ker-smack down an' snag hissef on dis stump! Please, suh, ter stop! hit gin me de flirtation er de heart ter see you do dat-a-way! I gwine go right 'long home dis minnit ef you don' quit dem gwines-on an' come down f'um dar.' She go on lak dat 'kase she ain' got sense 'nuff ter know he wuz jes' ez much at home up dar ez w'at she wuz on de groun', an' dat his tricks wuz jes' ez natchel an' easy fer him ez wat hern wuz fer her. De trouble wid her wuz she think she know hit all.

"Pres'n'y Mris' Jinny Wren, whar live up in de same tree an'wuz gre't fren's wid Brer Squ'l, she git tired er lis'nin' at Mis' Hyar's foolishness, an' she say ter husse'f, she do, 'I gwine mek up a song 'bout dat fool ooman an' sing hit out good an" loud, so "'t she"ll know someb'dy else year her gwines-on sidesen dat nice li’l Brer Squ'l whar don' 'spicion nob'dy. I 'low hit teck one ooman ter see daylight thu anu'rr. Dese mens don' seem ter be up ter de bizness.' 


\section{AT THE BIG HOLSE}

"Wid dat she cle"rd her th"oat a li"l an" sung a note er two ter git de pitch, an" den she stick her haid outen a hole in de tree an' sing out loud ' $\mathrm{z}$ she kin,

$$
\begin{aligned}
& \text { 'Squ't he jump f'um limb ter limb, } \\
& \text { Ol' Hyar' sot an' look at him; } \\
& \text { Ol' IIyar' say onto hussef, } \\
& \text { Dat Squ'l don' min' he hill hisse'f." }
\end{aligned}
$$

"And what did Molly Hare do then ?" asked Ned.

"Law, honey, she wuz dat mad at bein" overyearn by nu'rr ooman dat she jes" ga "rrd husse f toge'rr an" lit out fer home, same 'z I gotter do dis re'y minnit er my ol' man be sendin' one 'r de gran'chillen ter fin' me. Y'all better putt dem haids clean down on de pillers, fer y'ain' gwine year nu'rr wud outen my mouf dis night."

Here she discorered that little Kit had fallen fast asleep with his arms orer his head, and she stopped to lay them down, muttering to herself "'['m-mmph! jes' look at dat chil" wid his arms over his haid. Good thing I seed 'im. He mought er stayed dat-a-way all night, an den de goodness on y knows wat moughter happened to "im. "kase any pusson dat sleeps wid his arms over his head is jes' callin' down bad luck on hisse'f ery minnit er de time. Luck is sech a skittish sort e: thing dat I don' b'lieve in foolin' wid hit ner teckin' any resks. Some folks ain' blieve in signs, bat I bin watchin' 'em all my life, an' seed so many un 'em come true dat I gwine be on de safe side. fer ef dey don' come true 'bout one thing dey boun' come true 'bout nu'rr." 


\section{MR. HARE AND MIR. FLINT ROCK}

One evening the children heard the sound of a banjo in the cook-house and begged to be allowed to go out again and listen.

"Well. I declare." said their mother. "you children are getting so you don't care about being with white folks at all: your mother has to play second fiddle these days. I don't know what she is going to do for some little children to love her if it keeps up like this."

Kit ran to her. and, throwing his little arms about her. gave her a good hug, as if to reassure her. and Ted said they would have to make the most of their time. as they would presently go back home where there would be no negrnes and no stories, when he and the other two would try to "make it up to her."

"Tery well." said their mother, "since you are going to make it up to me, I suppose I shall have to let you go, but you must promise not to stay late."

They found Tim, the plow-boy, playing and singing for the benefit of the assembled company, among whom was Aunt Nancy"s granddaughter, Cassy. She had been off that day to the nearest country store to trade. carrying what she called a "haid-turn": a haid-turn being a small amount of produce, as much as could easily be carried on the head. which was to be exchanged for groceries or dry goods. Cassy was still decked out in 


\section{AT TIIE BIG HOLSE}

her best dress of black and yellow calico with a marrelous garnishing of flying red ribbons. It seemed as if 'Tim's song was especially addressed to the young lady, for, as he "picked" industriously and threw his head back and opened his mouth to its fullest extent, he kept his eyes steadily on her face, as if to note the effect of his song.

LI'L 'LIZA JANE.

"I got a house in Baltimo",

Li'l 'Liza Jane;

Po'ch behime an' po'ch befo',

Li'l'Liza Jane.

I got a buggy an' a hoss,

Li'l 'Liza Jane;

Come 'long, honey, an' be der boss,

Li'l 'Liza Jane.

I got a house an' a track er lan',

Li'l 'Liza Jane;

Shate yo' foot an' clap yo' han',

Li'l 'Liza Jane.

Come, my lore, an' go wid me,

Li'l 'Liza Jane;

An-a we will go ter Tennessee,

Li'l Liza Jane.

Cassy and Tim were not an openly acknowledged "co'tin-couple," and it was, therefore, not etiquette for her to encourage him too obviously, so she tossed her 


\section{MR. HARE AND MR. FLINT ROCK}

head and said that she had not come there to hear any "ol" co"tin"-songs," lut in the hope of listening to some stories. Eliza, the cook, as usual, took the part of the gentlemen. Said she, "Gal, de white folks cu'd git "long widout butter on der bread, but dey ain' wanter, an' we-all cu'd git 'long widout songs, but we ain' wanter. You spose yo' "pinion gwine mek any diff"ns ter Tim? Sho! g'way, gal, mens got sump'n else ter study 'bout sidesen w'at you does lak an' w' at you doesn' lak. But ef you right sot on yearnin' tales, mebbe we kin coax sis: "Phrony to faror the comp'ny."

Aunt 'Phrony said that she might be able to remember something if they would give her a little time in which to think it up, and Janey begged that it might be something about the much admired "Ol' Hyar"."

"Now, den," said the story-teller presently, "ef you wanter year 'bout ol' man Hyar'. I reckon I mought ez well tell you 'bout de time he met up wid Flint Rock, 'kase I kain 't 'member dat I has urer tol' you dat befo'.

"One time Hyar" he year dat de creeturs wuz gwine hare a big darrse, an' noration bin sont roun' dat de queschin wuz ter be 'cided w'ich un 'em have de fines' dress, an' he say ter hise'f, he do, 'L'mph! I gotter have a han' in dis thing, sho'. Dey kain't run dat 'sputation lessen I'se dar ter he'p 'em out. I ain' bin lopin' roun' de kyountry all dese 'ears fer nuttin', neener; I boun' you I gwine be ez well dress' ez de nex' un.'

"So he tuck an' primp hisse'f up ter look ez harnsum ez he kin, an' set off fer de darnse, an' he had hit in min' dat ef he run 'cross any creetur on de way whar have a finer suit er clo'es dan his, he gwine light in an' git hit way ef he kin. 


\section{AT TIIE BIG IIOLSE}

"ITe go clippin" 'Iong thu de kyountry, jes' ez full er mischicef ez a aig is er meat, an' w'en he kain't fine nuttin else ter do he tromple on ev'y po' li’l bug an' w'm he see an' switch de haids offen all de flow's ez he pass. He trabel an' he trabel an" he trabel, an' las' de night come down an' ketched him a fur ways foum de place whar de darnse wuz gwine be hilt, so he say ter hissef dat bein' ez dat wuz a strange kyountry an' he dunno de way re'y well, he bes' camp out fer de night.

"'Bout dat time he met up wid Flint Rock, a gre't big ol' man wid gray hya'r, settin' down by de side ur de road. He wuz so ol' dat de moss wuz growin' on his back, an' he wuz so full er rheumatiz fum settin' out in de damp dat he kain't move fu'm one spot lessen some one come 'long an' gin him a shove. He wuz a mighty sollumcholly an' sev'yur lookin' ol' man, but co'se Hyar' he wan't faze' by dat, so he walk right up in front ur him an' stan' wid his han's in his pockits an' say, 'Howdy, Wistah Flint Rock; how does yo' bones seem ter stan' de rackit dese days?'

"Flint Rock he 'spon', 'Howdy, Mistah Hyar'-I b'lieve dats you, ef I ain' mek no mistake. But I ain' 'zackly sho', 'kase my eyesight done fail me right much de las' 'ear er so. How does I fin' mysef? Mighty po'ly, suh, mighty po'ly. Ef you'd bin settin' out yer in de sun an' de rain an' de wind an' de wedder, in de fog an' in de damp ez many 'ears ez w'at $\mathrm{I}$ is you be chockfull er rheumatiz, too. Yit I ain' complainin', an' I mek out ter enj’y mysef yit. W'en I wuz younger an' went rollin' thu dis onfren'ly worl' I had a heap er fun an' seed a lot er sights, but now I done sottle down yer, an' yer I stay, an' things is lind er good ter de ol' man, 


\section{MR. HARE AND MIR. FLINT ROCK}

atter all. De li'l roung green things. furns an sech, der creeps up clost ter my footses an stays dar, an de lizzuds dey comes ont an lays down on my back ter wom derse fs in de sum. an de li"l bu ds dey lights on me now an' den an'stays 'long 'nuff ter say 'howdy' I jes wall my ere up at de sun ez I set yer. an he blink down at me right fren'ly an' wom up my back sos de mis'ry mos' quit me. De mosses dey is tryin' ter kiver me up sost I kain't feel de col wedder in my jönts. Tren hits wo'm de wind come 'long an' cool me off, an de rain gin me a washin' now an' den, an' lots er de creeturs stops ez dey go by an lays down in de shade er me ter git cool. an' I pass de time er day wid 'em an' git all de news. I jes" set yer an' look an 'lissen, an' I see an' year mo in you urer dream ur 'mongs' de bu'ds an' de beaseses an' de bugs an' de wums an' de flow's, 'kase de ol' man set yer so quiet der think he mus' be sleep, an' dey goes on same 'z ef I wan't roun'. Yas, Mistah Hyar', I cu'd tell you heap ef I wanter.'

". Well, sez de Hrar, sezee. 'dat suit my time 'zackly, 'kase I wuz kyountin' on spendin' de night wid you, suh, an I reckon you ain gwine kyare ef I build me a li'l fire right "long sile er you, so st I kin keep wom. De nights gittin right chilly.

"Dat dey is:" sez Flint Rock, sezee; 'I got a crick in my back a-raidy. Goodness on y knows how I gwine git thu murr winter. I "srec one dese yer hard freezes gwine come 'long an' jes" 'bout break me all up. I look ter see dat happen. You g“long an' build dat fire an' we kin set an' talk an' me wo'm up my ol' bones at de same time."

"Hyar" he build de fire, an" git him a piece er wood, 


\section{AT TIIE BIG HOCSE}

an' all de time Flint Rock wuz gwine on talkin', Hyar' he wuz settin' dar wu'klin' way on de wood.

"Lis' Flint Rock he say, sezee, 'Mistah Hyar', please, suh, ter 'scuse de cur'osity ur a ol' man, but mought I ax wat is dat rou mekin"?

"Hyar" he say, 'W'y, sut'n'y, suh; dish yer's a maul an' dis a waidge.'

"Flint Rock he 'spon', 'Tas, so 'tis, so 'tis, I see dat now. You mus' 'scuse me axin', 'kase my sight so bad. But mought I ax you, suh, wy you mekin' dat maul an' dat waidge?'

" 'W'y, suh,' sez Hyar', sezee, 'I'se a mighty indush'ous man, an' hit go 'gins' de grain fer me ter set an' hol' my han's, so w'iles I lissen at you I jes' do dis ter keep my han' in an' pass away de time.'

"Pres'n'y Hyar' 'scuse hisse"f an' say he mus' git nu'rr li'l bit er wood, an' den he go off an' fin' hisse': a place whar he kin hide w'en he wanter. He fin' a li'l kyare, an' den he walk back ter de camp, an' dar wuz ol' Flint Rock, fas' asleep by de fire. Wid dat he tuck an' tuck de waidge an' putt hit at Flint Rock's year an hit de waidge a monstous clip wid de maul, wow! an' ol Mistah Flint Rock bus' in mo'n a hunderd pieces. De pieces flewed high up an' 'menced ter fall thu de air. swish! zish! zoum! An' w'en he see dat, Hyar' lit out f"um dar an' hid hisse'f in de kyare.

"'Tmph!' sezee ter hisse'f. sezee. 'I done save' de ol' man de trouble er waitin' fer de fros' ter break him up. He orter thank me fer puttin 'him outen his mis'ry fo' de real col' wedder come on, dat's de trufe.' Atter w'ile he say. 'Lan' er libbutty! dis slow wu'k waitin' yer in de dark fer ol' Flint Rock ter git thu fallin'. W'en I 


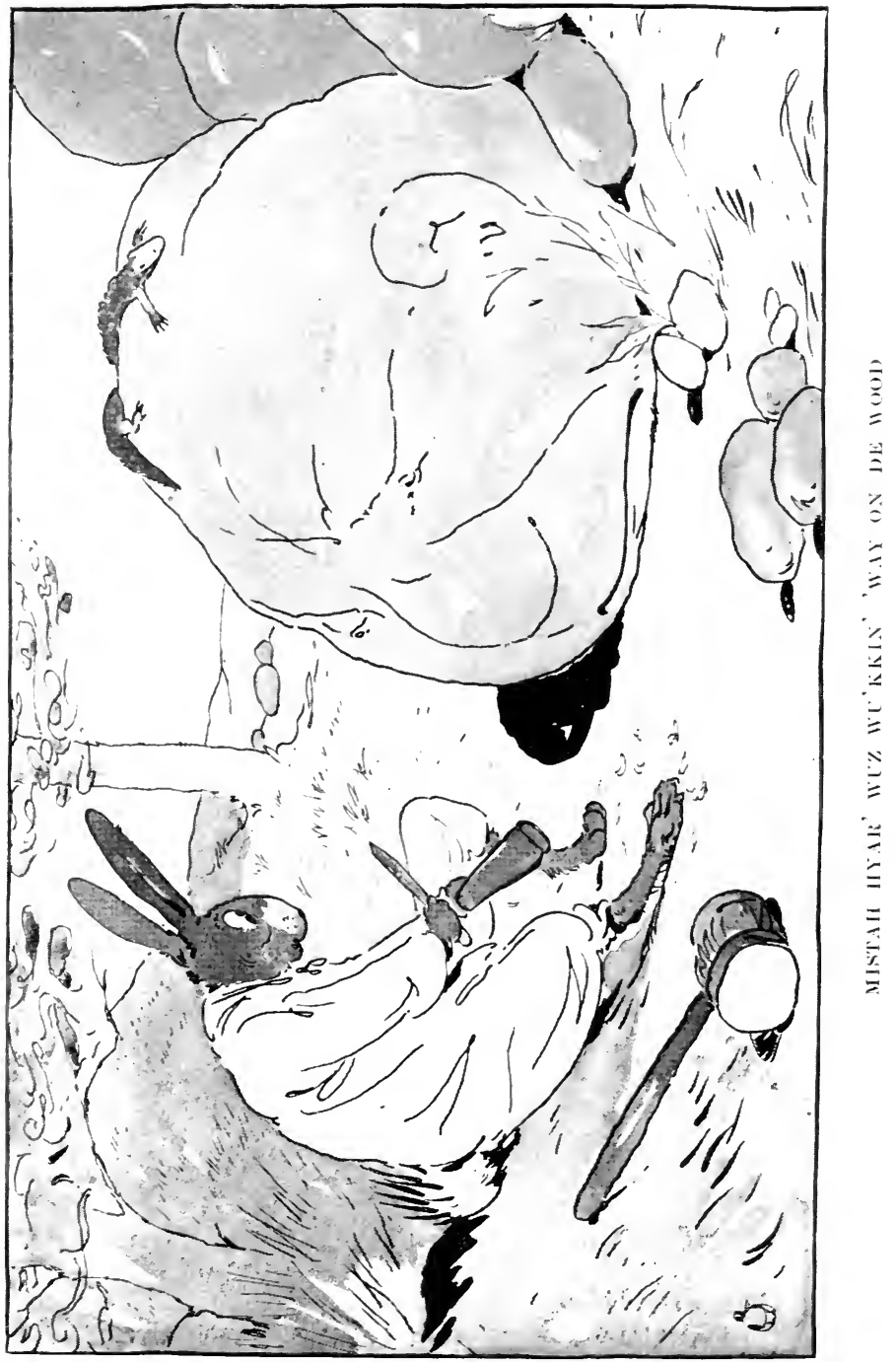





\section{IIR. HARE AND IIR. FLINT ROCK}

fotched "im dat lick I ain" have no notion he gwine fly in li'l bits lak dat, but hit right hard ter tell wat some folks grime do 'twel you tries 'em. 'Pears ter me he orter be thu fallin' by now; I gwine look out an' see.'

"Irid dat he poke his haid out. De las' piece wuz fallin', an' suz! ef you'll b'lieve me, hit tuck ol' IIyar' plumb on de een' er de nose, and dat huccome he bin gwine roun' wid a bent nose urer sence, an' all time drorin' hit up an' down lak he 'feard nu'rr rock gwine teck him on the een' ur hit mos' any minnit."

"Why. Aunt Nancy told us the reason Mrolly Hare"s nose worked all the time was because she got it frozen," Ned exclaimed.

"Il"at I know "bout Xolly Hyar"?" said 'Phrony, with scorn and some indignation. "How many mo" times is I gotter tell you I ain' know nuttin' 'bout no Molly Hyar"? Dis de one de Injuns tell 'bout, an' he ain' nuver have no wife 't all, fur 'z I know. I done tol' you he wuz a ol' bachelder'; ef he'd had a wife ter keep 'im straight he oon bin in so much mischief." 


\section{MR. WILDCAT GOES TLRKEY-HUNTING}

"Landy !" said Cassy, after the story of old man Flint Rock, "ef dat Hrar" ain" beat all creashun. Scem lak he got mo" gumption dan all tu'rr creeturs putt toge "rr. sho! gimme a man lak Mistah Hyar" whar got plenty er git up an "sperit to "im." And she tossed her head and looked scornfully at Tim.

"Look yer, gal!" said 'Liza. "you dumno w'at you talkin' 'bout. Ef you kin lay yo" han" on a good, stiddy man, you better teck him an thank yo" stars. Dese yer lively, gaily folks lak Mistah Hyar" does well "nuff ter pass 'way de time wid. now an' den. but w'en hit comes ter livin' wid 'em, dat's anu 'rr marter. Sidesen dat, dey git kotch up will er'y now an' den, jes' lak sis' 'Phrony bin tellin you. Mistah Hyar" wan't so smart but w'at he git his nose good an" smash."

"Wellum." said Tim. in his slow drawl, "I bin studrin" heap 'bout de ereeturs an' I bin wmmerin' ef dey don' none un em play dese yer tricks asseptin' ol IIyar."

"Bless yo' soul," answered 'Phrony, "dey all un 'em had pranks, mo" er less, some mo", some less. Now dar Mistah Sly-fox, he kind er pranki-h sometimes, an' Mistah Tarr'pin, too, an' I 'members one time w'en Mistah Wil'cat tucken hit in min' ter play a trick, dough to be sho' dat wuz sort er in de line er bizness, w'en he wuz out fer de puppus er gittin' hisse'f a dinner." 


\section{MR. WILDCAT GOES TLRKEY-HLNTING}

Coonie, the house-boy, was sitting on the hearth, leaning against the chimney jamb, hearl thrown back, more than half asleep. but at the mention of dimner he sat up and rubbed his eres and askerl, sleepily: "Dinner? Dimner raidy? Maw, is I year you say dimner ?"

"Yaw, dat you ain"," said 'Liza. "I done fill you up wie? vittles no mon a hour ago, an' rer you is axin' fer mo'. 'Pears lak I kain't keep you filled; you is wusser dan any ol wileat dat urer tromped de woorls. Go on. Sis' 'Phrony, an' tell us 'bout Mistah Wil'cat an' his dinner."

"I done tol' you he wuz out fer de puppus er fillin' up." 'Phrony went on, "an' he wuz dat hongry hit "pear ter him he wan't nuttin' but one big holler f'um de top ur his haid ter de tip ur his tail, one r dese yer hollers whar jes natchelly mek a man git up an hmmp hisse f ontwel hits filled. He go traipsin' up an down. lookin' behime er'y bush an' up er'y tree an' rit he kain't fin' nuttin'. Las' he got so mad he 'gun ter gnash his toofs an'spit an' snarl an' lump his back up an' rub gin' de trees, an' all de time his ẹes wuz shinin' lak plumb balls er fire, an' he wuz dat rantankerous dat no pusson 'ud want ter meet up wid

"Bout dat time he seed a flock ur wil'-tuklies, an' he 'low ter hisse'f dat tukkey "ud jes' 'bout fit de size er his mouf, dough he ain' knowin' how he gwine git at 'em. He crope up an' he crope up, an' las' he wuz near "nuff ter watch "em. Dey wn hol'in' a darnse, de funnies' you urer see er year tell bout. Der tails wuz all spread out lak a fan an' de gobblers wuz all puff' up an' struttin' fit ter kill. w'iles de ladiz wuz mekin' gre't 'miration over 'em an' follerin' atter 'em, steppin' high 


\section{AT TIIE BIG HOT'SE}

lak dey wuz trompin' on aigs, an' gwine roun' an' roun' in a succle, lookin' mighty proud an kyar'yin' der haids high in de air an' balancin' wid der necks at er'y step.

"Wil'cat grin so't he show er'y toof in his haid, an' he say ter hisse'f, sezee, 'Tm-umph! some dem ladiz gwine laugh on de wrong side der moufs 'fo' long. Ef dey on'y knowed wat comin' dey'd quit dat foolishness an' use dem laigs ter git outen dis. Good thing fer me dey is fools. I reckon I'd have mighty po' pickin's ef der wan't so many fool creeturs gwine up an' down de face er de yearf.

"De tukkies wuz darnsin' down in a li'l holler, an' Wil'cat he crope roun' ter de top er de hill an' tucken de game bag whar he bringed wid him, an' crawled inter hit. Den he turnt hisse'f aloose an' rolled over an' over down de hill, bimpitty, bumpitty, bampitty, bim! an' lan'ed right in de midse er de tukkies.

"Dey wuz dat 'stonish dey ain' know w'at ter do, but w'en de bag stan' up on een' an' 'gun ter r'ar an' charge roun', dem po' creeturs wuz plumb stunded, too 'stracted eren ter run. No wumner dey wuz dat-a-way, fer ur all de doin's anrb'dy urer see. Mistah Wil'cat's tuck de lead. Ter see nuttin' but a ol' bag jumpin' high in de air an' turnin' somersets an' rollin' on de groun' an' den gittin' up an' doin' all sawts er steps. dat 'ud 'a bin jes' too much fer any pusson, let 'lone dem fool tukkies.

"W'en he git 'em kind er daze' an' 'stracted he step outen de bag an' he say, sezee, 'Howdy, ladiz an' gemmen, howdy! I hope I sees you well an' in de enj'ymint er good healt'. I seed you down yer havin' a pow'ful good time, an' I had de insurance ter jine you an' give you a eenvite ter teck a roll down hill in dish yer bag er 


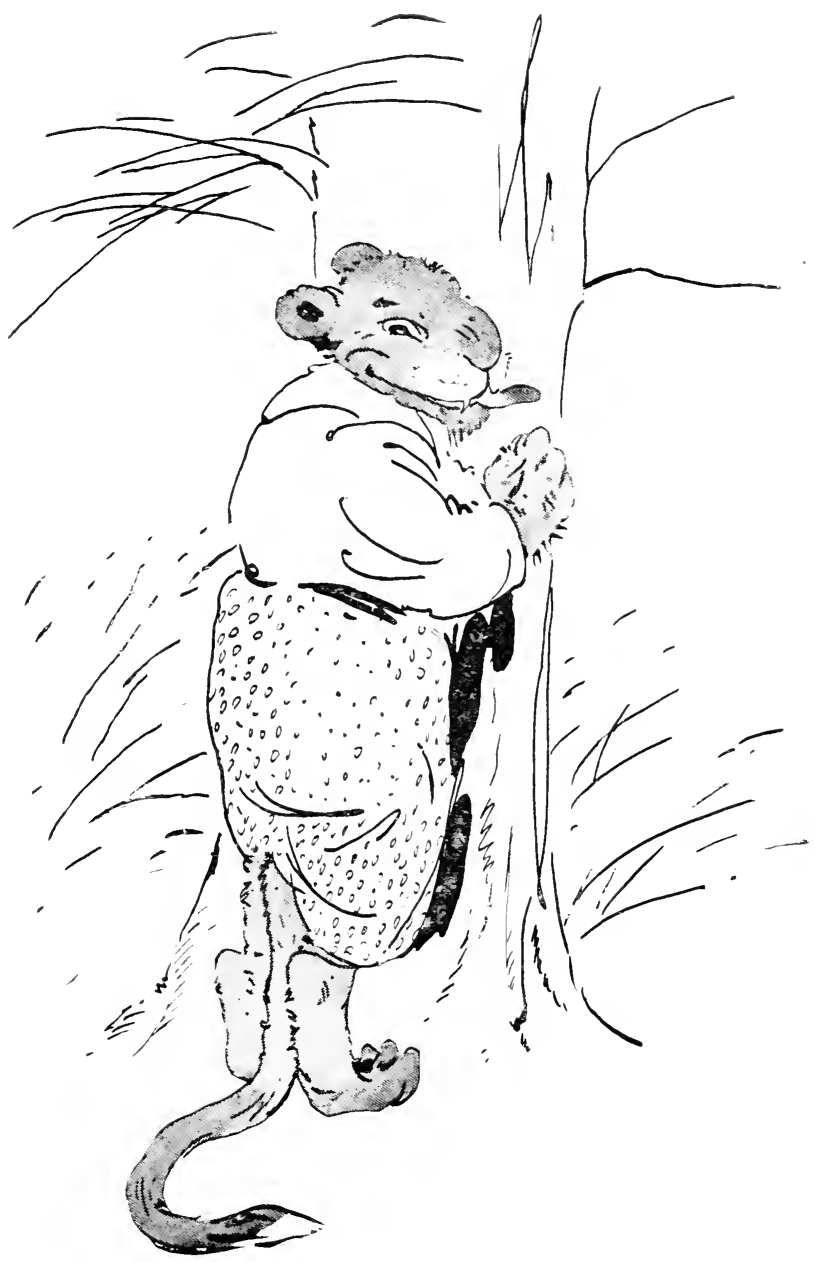




\section{AT TIIE BIG HOTSE}

mine. You-all think darnsin' is fun, but hits mighty po" shuncks "long side er de sport er rollin" down hill in a bag. Dem whar ain' tried hit dumno nuttin' 't all "bout hit, an' w'ats mo', dey ain' guine know lessen dey tries hit. Come one, come all! Step up, ladiz an' gemmen; de fus' one in gits de fus' ride. I'll tote you ter de top er de hill an' give you a shove down. Dellaws! w'at yall hang back fer? I'all so p'lite I 'spec' you 'feard you be onmannerly ef you gits in fus'. Nemmine 'bout dat, I'll gin you ev'y one a whu'l. Step in, step in, my time mighty val'able. I gotter be movin' outer dis 'fo' long an' I dunno w'en I gwine come dis way ag’in. You dunno wat yer missin' ef you let dis chanct slip by. Sho'! I thought y'all had sense 'nuff ter know a good thing w'en you seed hit.'

"Two er th'ee er de ladiz mek up der min's dey'd lak ter try de new sport, jes' out er cur'osity, an' dey went tippin' up to'des Mistah Wil'cat whar he'z hol'in' de mouf er de bag open. Jes' den a ol' gobbler step up befo' em. 'Twuz Mistah Wi'yum Wil'-tukkey, jes' a struttin' an' a puffin'. His fedders wuz shinin' in de sunlight lak brunze, an' his laigs wuz raid an' his neck blue, wid warts stannin' out over hit, an' he have on de fine by'ud an' wattles whar he stole 'way f'um Mistah Tarry-long Tarr'pin. He sut'n'y look harnsum, an' he sut'n'y ac' lak he knowed hit. He step up befo' de ladiz an' bresh 'em ter one side an' say. 'Naw, ladiz, rollin' down hill ain' no fitten 'musemint fer wimmins; leave dat fer de mens. G'long home an' tend ter y'all's famblies. Ef de trufe wuz knowed. I 'spec yo' chillen is all scattered out inter de bresh by derse'fs dis ve'y minnit 


\section{MR. WILDCAT GOES TLRKEY-HL NTING}

whar sump'n boun' ter git 'em. 'Spectable wimmins darnsin' at yo' time er life! Wimmin-folks ain' got no bizness w "ay f’um home, nohows; sho' ter git inter mischief an' do sump n nu 'rr dat ain' fitten fer 'em ter do. Set still an "hare yo"se"fs an let de men-folks tend ter de runnin roun' Lawdy, lawdy! I kain't see wy you all de time wantin' ter do lak de men-folks does!"

"Wid rat he flounce hissef inter de bag, an' Wil"cat he dror de strings in a jiff, an' de way he lit out f"um dat place inter de low-grom ${ }^{\circ}$ wuz a caution ter de beholdens. Ez he go chargin thu de bresh he rell back. 'Good by. ladiz, saw' y ter run off wid de haid er de fambly. but yall mu'n fault me fer dat. He would be de fus' in de bag. Dat w'at he git fer not bein' p'lite 'nuff ter let de ladiz have fus' chanct. Ladiz fus' ev' $y$ time, I ses! Dat's me. Whoop-ee! good-by, er'yb'dy! see y'all ag“in some urr time w'en I git hongry.'

"De tukkies dey flewed up wid a gre`t cryin' an' flutteration, an' dẹ wan't seed no mo' in dat place, I kin tell you. ner nob'dy urer bin able ter suade one ter putt haid in a bag sence dat time; dey done member hit too well. Dey flewed off gobblin' an gabblin', an' one ol' un she say ter turrs. 'Th-huh: "pears lak some er de mens gits inter trouble w'en dey g way f'um home, well ez de wimmins. I don see but wat we got jes' ez much sense ez dat ol' Wi'rum Wil'-tukkes, atter all. I reckon he d lak ter change places wid us dis re'y -minnit.'

"Nu'rr ol' un she say, 'Yas, lawd! dat de way wid de mens. all time excusin' we-all er cur'osity, w'en. ef de trufe wuz knowed. we kain't hol' a cannel to 'em in dat marter, y'all done seed dat fer yo'se'fs right now. But 


\section{AT TILE BIG IIOTSE}

I reckon ef he euil come back yor dis ve'y minnit, w'ich prate de Lawd he kaint. he id say dat we done putt de notion in his haid by steppin' up ter de bag.'

“An' dey all flapped der wings an' ses, 'Yas, lawd!" 'Trufe, too!' 'I boun' you!'” 


\section{IIR. FOX TLRAS FARMER}

When Aunt Phrony had finished telling how the Wil'cat caught Mr. William Wild-turker it was Aunt Nancys turn to entertain the company. The children reminded her of a story she had once told them about certain shrewd practices of Mr. Hares by which he circumrented Mr. Sly-fox, and they wanted to know why it was that he alwars got ahead of the other animals.

"I dunno jes" huccome so." she said, "but 'pears ter me dem gre"t big eres an' dem long vears is w'at he'p him out; he see an' year mo" wid 'em in a minnit dan urr folks knowin' in a week, an' den he set up on his ha'nches an' wu'k his nose. sort er so, an' study w'at use he kin putt hit all to in de way er meanness, all de time lookin' jes' ez meek an' innercent ez a lamb."

"Tere he and the fox friends afterwards?" asked Ned. "I bet I'd a-thrashed him good if he came fooling around me."

"Tell," said Aunt Nancy, "dey kind er, sort er patch hit up, but der feelin's ruz wu'kkin" un'need all de w"ile an' boun' ter riz up ter de top sometime, same 'z dish yer barm I putts inter de wheat-bread. But Fox he "low ter hisse'f dat mebbe he kin mek use er ol' Hyar' an' git evin wid him at one an' de same lick, so one day he call roun' at ol' man Hyar's, an' atter dey pass de time er day, he say, 'Mistah Hyar', I gittin' mighty tired er dish 


\section{AT THE BIG IIOLSE}

yer huntin' an' fishin' bizness. Hits hard wu'k, suh, hard wu'k. an' my ol' ooman she gittin'. lind er raspy 'kase I ain' bring home mo' vittles ter her an' de chillens. I tell her dis mawnin' I b'lieve I gwine go in de farmin' bizness, an she tucken ter de notion, but she say I kain't do hit by mrse'f, dat I 'bleeged ter have he'p, an' she 'low, she did, dat mebbe me an' you cu'd go in cahoots in dis marter, fer hit well knowin' ter er'yb'dy dat you is a gre't han' fer all sorts er gyardintruck.'

"Right dar Fox grin ter hisse'f, 'kase hit wuz de sho''nuff trufe dat Hyar' wuz fond er gyardin-truck, mo' 'special w'at come outen urr folkses gyardins.

"Mis' Molly Hyar' she wuz doin' chores roun' de house, stoppin" now an" den wid her han's on her hips ter lissen at 'em talk er ter cuff one'r de chillens. fer Bunny an' Honey wuz rollin' in de ashes on de ha'th, an' Jumper an' Thumper wuz playin' leap-frog, an' Winker an' Blinker wuz tryin' ter pull de water-pail over. She go roun' behime her ol" man an' hunch him in de back an' whusper in his year, 'Spunk up. now, Mistah Hyar', an' tell him you go in cahoots wid 'im, 'kase dish yer's yo' chanct ter fool 'im ag'in.'

"So Hyar' he do lak he studyin' 'bout hit, an' las' he say, sezee, 'Well, I dunno 'bout dis gyardin-truck, 'kase hit pe"ish out in de col' wedder: I bin studrin' 'bout lettin' dat go an' raisin' me a cawn crap dat las' all thu de winter.' He say dat 'kase he knew mighty well he gwine live offen urr folkses grardins in de summer, but he ain' knowin' w'at he gwine do, 'zackly, 'bout vittles fer de winter.

"Fox say dat he 'gree ter de cawn crap, an' den Hyar' 


\section{MR. FOX TLRAS FARMER}

lean his haid on his han' an' do lak he study some mo'. Las' he say, sezee, 'I dunno w'at we gwine do 'bout plowin: De groun" orter be bruk up dis ve y day, an' yer me all lame" up f"um dat las' spell er rheumatiz. I be mighty saw'y ter see you start in by yo"se"f, but I kain't see de way outen hit. I 'low de wedder gwine be li'l wo 'mmer atter w'iles an' den I kin light in an' do de wu'k er two mens ter mek up fer dis.'

"Fox kind er "spicion dat IIyar" was foolin' him, but he wuz de green han' in de farmin' bizness an' hatter be showed how ter do de wu'k, so he kain't say nuttin'. He tromp all day over de rough clods, drivin' de plow, 'twel his han's wuz all blistu'd, an' he wuz dat tired he hatter hol' onter de plow hannels ter keep f'um drap'pin. All dat time ol' man Hyar' wuz settin' at home in de arm-cheer, smokin' an' teckin' hit easy. He 'ud grin ter hisse'f er'y now an' den, an' w'en Mris' Hyar' ax him w'at de marter, he 'ud tell her, 'Oh, nuttin, nuttin' 't all, jes' a li'l spazzum er de jaw, 'twon't las', don' 'mount ter nuttin', nohow.' But Mis' Hyar' too smart ter be tucken in dat-a-way; she knowed sump'n wuz up, an' she go off moufin' ter husse'f, 'Shucks! ef dar's one thing I hate mo'n anu'rr hits ter see a fool man settin' up laughin ter hisse'f w'en nob'dy else knows wat de joke is; looks too triflin' fer any use. 'Pears lak he muss' be laughin' at you er else at de empty air, one $\mathrm{r}$ de two, an' nob'dy but a plumb ijit gwine do dat las'.'

"Tren plantin' time come, Hyar' drug hisse'f out ter de fiel' an' let de cawn run thu his fingers lak he'z so weak he kain't hol' onter hit. Den. all ter onct, he wuz tucken wid a fit er de shivers an' trimmle lak a aspum, 


\section{A'T TIIE BIG IIOUSE}

an' he say he b'lieve he gittin' de bone-break ferer, an' he 'bleeged ter go home an' git on de baid.

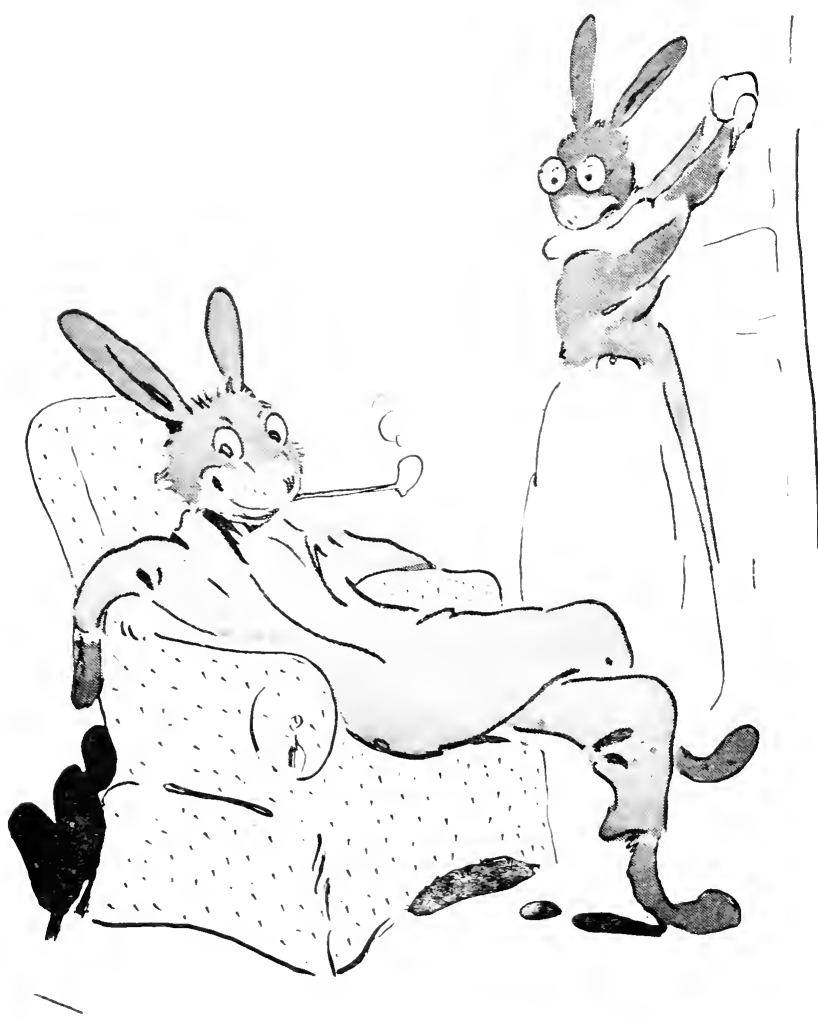

"W'en de time done come ter wu"k de carn, Hyar" tucken a hoe an "g.longed ter one een" ur a row an' tol' Fox ter begin at tu'rr een', an' den dey'd meet in de middle, an' dat way each man be sho' ter do half. I let you 


\section{IR. FOA TLRAS FARIIER}

know he wuz mighty slow at his een" er de row an " w' der wuz bout ter meet. he mence ter sing songs an" tell tales an "ut sech shines dat ol Fox ain" notuss how li"l wa'k bin done at dat een" er de line. W"en Hrar" see he done got Fox": min clean offen de wuk, he tuck him by de shoulder. right fren "ly an familious, an' turn him roun' tu'rr war. an' den he say: 'Tell, well, ol man. rou has" come near half-way. but you does mighty well fer a green han. Jes" you g*long back ter de een" er de nex" row. an "w" we meets ag "in, lessee ef you kain't git li"l mo' nearer ter de middle.'

"Ef Fox had look behime "im he ud a knowed he bin fooled. but he ain" have de sense ter do dat, so he go trudgin an' trampin all de way down de row, wiles Hyar" he gin a hop. skip an" jump an wuz back in his place in a jiff, "kase he ain" have fur fer ter go.

"Dey kep' hit up dat-a-ray ontwel shuckin" time, w'en dey wuz ter 'ride de crap. Den Hyar' he say, sezee, 'I done had de mos" spe yunce an sidesen dat I is good at figgers, so I reckon I bes" do de kyountin" an' let you do de shuckin $\because$ so Mistah Fox he sot down below an' pullt de shucks offen de cawn an' th'owed de rears up ter ol Hyar, whar wuz stannin' up in de cawn-crib ter ketch"em.

"He kyount straight fer li"l w"ile, "kase he seed Fox cuttin' his ere up at "im er. now an" den, but pres"n' w'en he think he got "im offen de scent an" de cawn "gin ter pile up roun' "im so"t Fox culden see "im re’ well, he lit out inter meanness ag"in an' start ter kyountin' wrong. All de time he wuz singin' a ol'-time shuckin'-song whar run lak dis: 


\section{AT TIIE BIG HOLSE}

\section{'II ushi-o up an'-a-husti-o doun, An'-a-hustio, hustio all aroun', Turn, O ladiz, turn, turn, Somebody's at de winder.'}

"W'en he sing 'husk-o up,' he kotch a year er cawn, an' w'en he sing 'husk-o down,' he mek lak he th'ow hit on Fox's heap, but stidder dat he pass hit roun' behime him w'en he say 'husk-o, husk-o all aroun', an' w'en he say 'Turn, O ladiz, turn, turn,' he drap hit sof'ly onter his own heap. He do dat er'y urr time, an' I ses ter you dat w'en dey got thu, ol' man Fox come out at de li'l een' er de hawn, wid a pow'ful po' showin' er cawn.

"Fox scratch his haid an' feel mighty jubous. He say, 'Ter I is, done wu'k day in an' day out, rain er shine. Done plow, done sow, done hoe, done harres', an' dish yer li'l heap er cawn all I got ter show fer hit. Sump'n wrong, sump'n 'bleeged ter be wrong, yit I kain't jes' prezackly putt my paw on hit. I boun' ol' Hyar' know mighty well w'at 'tis. But 'tain' no use ter ax him ques'hins ner argyfy, 'kase he boun' ter slip outen hit, somehow. I wish 't I had 'im yer dis ve'y minnit, de trash-er-de-worl'! I boun' I'd teck de natchel hide offen “im.'

"Jes' 'bout den Hyar' come sa'nterin' 'long an' year de las' wu'ds whar Mistah Fox done say. 'Who dat you gwine teck de hide offen him ?' sezee. 'Oh, nuttin' 't all, Mistah Hyar', nuttin' 't all: jes' one'r my chillens whar bin talkin' up sassy ter his maw w'iles I bin 'way f'um home, farmin'. Chillen gittin' mighty 'sumptious dese days, Mistah Hyar'; mighty 'sumptious, suh; an' we-all mus'n' spare de rod lessen we wants ter spile de chil'.' 


\section{IIR. FOX TLRAS FARIIER}

"In co'se Hyar' wan't tucken in by dat talk 'bout de 'harishness er Mistah Fox's chil', but he pass hit orer' an' say, mighty fren'ly: 'Jistah Slickry sly-fox, how 'bout dat 'tater crap ur ow'n? We mus' be gittin' dat in soon er Jack Fros' gwine come 'long an' git de good ur hit befo' we-all.'

"Dat wuz de time Fox think he see de chanct ter git ahaid er ol' Hyar'. He wuz dat green 'bout farmin' he ain' know dat de good part er de 'tater crap wuz un'need de groun'; he think de good part's on top, same ez wid de cawn crap, w"ich wuz de on"ies one he know anything "bout; so he say, "Well, Mistah Hyar", ef hit be "greeable unter you, I'd lak ter spar' you all dat kyountin' bizness you done went thu wid wid de cawn, an' so I ses le's we 'vide up de tater crap dis-a-way: me tek all dat's in sight, you tek all dat's outer sight; me all dat's 'bore de groun', you all dat's un'need hit.'

"Hyar' wuz dat tickelt he kain't keep his face straight, so he turn his back an' say, 'Scuse me jes' one minnit, Mistah Slickry, I got a bite, an' I'se 'bleeged ter tend ter hit.' His sides shuk so't he'z feard Fox might see 'im an' back outen de bargum. But Fox so please' wid de notion er comin' hit over ol' Hyar' dat he ain' notuss nuttin'.

"Hyar' turn roun' pres'n'y an' do lak he study li"l bit, an' den he say: 'Mistah Fox, I reckon I hatter 'gree ter dis 'rangemint, fer you done wu'k right fait'ful, hut "pears lak hit kind er one-sided, "kase er"ybily knowin' dat 'tater-tops mek mighty tasty greens, same time 't dey knows de wuf"lessness er de roots. Nemmine, I gwine do lak you sez.'

"Den Fox go all thu de 'tater-patch cuttin' off de 161 


\section{AT TIIE BIG HOLSE}

tops, an' Hyar' foller 'long atter 'im diggin' up de roots, an" bofe un 'em wuz so please" wid w'at dey doin' dat dey hatter stop er'y now an' den an' go off li"l ways ter langh. Den dey"d come back an' be mighty sof"soapr, an offer ter hep one nu'rr, an' den wa k on 'twel dey 'bleeged ter bus' out ag in. Las' dey wuz thu, an 'Fox went trundlin' off home, staggerin' unner de gre't load er 'tater-tops he truz kyar'yin' on his haid. W'en he git dar he wish he done stay 'way, fer Mis' Fox she know mo' 'bout sech ez dat dan he' ol' man did. She say, "I"'at you got dar, all wilt" up?"

"He say: 'Tater-tops. Mrek fine greens.'

"Den she jes" natchelly putt her mouf on 'im an' let "im know w"at she think.

"'I boun' dat wufless Hyar' done tol' you dat,' sez she, 'an' w'at's mo'. I 'low dat ol' ooman er his'n, dat ol' Molly Cotton-tail, done putt him up to hit; drat dat ooman!" sez she. 'Te settin' home yer slarin' fer you an' de chillens w'iles you off on dat farmin' bizness, an' now we-all gotter go hongry an' let dem hyar's nibble up all de taters you done w'k so hard ter raise. W'at is I done dat I hatter be tied to a man so onignoran" he dumno 'tater grows un'need de groun', an' so shif'less dat he let me an' my chillens go hongry? I reckon you bes" let farmin "Tone an" go back ter yo" huntin" an' fishin', dat is. ef you got sense 'nuff ter keep ol' man Hyar" f'um gittin' han's on erything. atter you done bag hit.'

"He ain" say nuttin"; he wuz dat outdone he kain't eren jaw de ol' ooman. but f"um dat time ontwel dis day an' gineration, no pusson urer year tell er Mistah 


\section{MR. FOX TIRAS FARMER}

Slickry Sly-fox meddlin wid farmin”. Ityar’ done kyore him er dat.

"An" now I gotter moser home." added the old woman with a huge yawn which she politely. but rainly, tried to smother. "an I reckon you chillen better be movin', ton, er de San 'Tan gwine ketch you betwix' yer an' de house, an you won't git home dis night." 


\section{CANADI AND THE WOLVES}

One day the children dropped into Aunt 'Phrony's cabin and found her sitting before the fire with her dog, Snap, curled up at her feet and her quilt pieces lying all about, engaged in putting together a gorgeous and complicated pattern which she called the "Sunflower Patch." Snap was the lean and ill-favored hunting dog that lived with Aunt 'Phrony on terms of armed neutrality; when she showed him the broom, he showed her his teeth, and the matter under dispute was usually dropped then and there. She valued him not for his character, but for his accomplishments as a hunter. He valued her as the giver of food and shelter and apparently there was no love lost between them; yet with that curious sort of loyalty which often prevails in families, Aunt 'Phrony would permit no one to asperse her dog but herself. It was not safe even to agree with her when she chose to speak her mind about him. When she stirred him with her foot to make room about the fire for the children, he gave an angry snarl and got up and walked off with an air of injured dignity which suited his style of dogship exactly. No doubt he knew that such was the case, for he chose to wear that manner the greater part of the time and only unbent in the excitement of the chase.

"Plague take de dog," said his owner, "dar's no livin' $16 t$ 


\section{CAXADI AND THE WOLTES}

wid de onmannerly beas'. He gits mo cross an' reringeful all de time. Ef he live "long "nuff I "spec" he git ez bad ez de wolfs whar useter wh fer Canadi. 'Pears ter me dat de wolfs an' de dogs is mighty clost akin, anrhow.

"You ax me who wuz Canadi? Well, now, he wuz in a ol', ol' Injun tale whar I useter year w'en I wuz a li"l gal in Nawf Ca liny. Canadi he wuz a gre thunter: sech a gre't man an' a gre't hunter dat de people ain' b'lieve he wuz 'zackly human. His name means, in de Churryke talk. 'Lucky fer Game.' De big game an' de li'l game he cu'd fine an' kill 'em all. nemmine how dey hide demse'fs 'way f'um "im in de woods an' de mountains an' de kyares, an' er'y one er de bu'ds an' beas'eses an' de fishes wuz 'feard er Canadi; dey wuz dat.

"De wolfs and Canadi wuz gre"t fren's; 'deed dey wuz all his sarrants an' he wuz de marster an' dey min' jes' w'at he say. He useter go out an' fin' de game, an' de wolfs dey"d wait an' ketch hit an' bring hit home. Dey's gre't on dat. Dey kin run an' run widout gittin' tired er losin' der wind, an' dey kin run down mos' anything dat run on laigs. So w'en dey wanster ketch some creetur dey jes' gits toge 'rr an' runs an 'runs him ontwel he's all beat out an' stove up, an' den dey nabs “im. Dey sut'n'y is a mean lot, mean ez dey kin stick in der skins, wid der ol' green eves an' der long tongues lollin' out an' der gre't toofs a-shinin'. 'Scuse me f'um meetin' up wid 'em atter dark. But Canari he wan't 'zackly human. so he wan't 'feard un 'em: 'deed dey' wuz 'feard er him an' dey ain' dast ter disobey him.

"One day he call 'em ter come an' go wid 'im. 'kase 165 


\section{AT TIIE BIG IIOTSE}

he wuz gwine out atter big game an' he want 'em ter foller clost. He ain' feed 'em den, "kase he knowed dey hunted better w'en dey wuz hongry, but he alluz gin em a part er wat dey brung home. Well, dey went on an dey went on, up inter de big mountains, an' las' Canadi come 'cross a turr'ble creetur whar live in a kirare, an' he lef' de wolfs ter skeer hit out an' fetch hit to 'im, an' den went on 'bout his bizness.

"De wolfs dẹ' skeered de creetur out an' lo an' behol' 'twuz a gre't sarpint. But, bless goodness, he ain' have no notion er gittin' outer de way : he jes' come a-r'arin' outer de kyare an splunged right inter de midse er de wolfs, an'I tell you dey had hit den. De fur flewed an' de wolfs howled an' de sarpint hissed 'twel de mountains plumb shuk an' de stones come a-rollin' down de sides. Las' de sarpint bit one'r de wolfs an' kilt 'im, an' de res' got skeered an' went lopin' off an' lef" 'im dar.

"Dat wuz de fus" time dey fail sence dey bin doin' huntin' fer Canadi, an' dey ses ter one nu'rr, 'Canadi mus' 'a had a han' in dis.' Dey knowed he wuz a gre't cunjerer an' dey 'spicioned dat he done putt a spell on de wolf whar got kilt. Der study 'bout hit an' study 'bout hit an' git mo' an' mo' 'spicious 'twel las' dey' 'clar' dat dey sut'n'y gwine revinge derse'fs an kill Canadi, an' den dey 'gun ter lay der plans ter do dat.

"Canadi he wan"t a cunjerer fer nuttin', an' he soon foun' out w'at dey wuz up ter, an' he call 'em all roun' an say. sezee: "My fren's ain' I bin yo' marster fer dis long time, an' ain' I treat you de bes' I knows how? Ain' I p int out all de game ter you an' ain' I gin you all a sheer ur hit? Ain' I treat you fa'r an' squar'?' 


\section{CANADI AND THE WOLTES}

"Dey hatter own up, 'kase 'twuz de trufe, an' dey look sort er 'shame' er derse'fs. Den Canadi go on an' he say, sezee: 'Twuz yo' own foolishness done de mischief, dat w'at 'twuz. You know de way I done l'arn you ter hunt is ter pussue atter de creetur 'twel hit all tire' out an' den s'roun' hit an' all fall 'pun hit toge 'rr. But dat sarpint wan't tire' out a-tall, an' w'en you seed hit show fight, den wuz de time w'en you orter done de gitten outer de way. I ses ter you, an' I don' want you ter fergit hit, dat hit's a gre't thing in dis worl' ter know jes' w'en ter run. Hit teks good hoss-sense ter know dat. Fools fights befo' dey thinks, an' tu'rr folks thinks befo' dey fights. Quit dis 'harishness an' cle'r ont 'twel I wants you, an' don' lemme year no mo' 'bout layin' plans ag’in me, er I putt sech a spell on you dat you'll ketch de nuver-git-overs.'

"De wolfs went slinkin' off, an' fer a li'l w'ile dey thought 'twuz jes' de sarpint dat kilt der fren', an' so dey ses: 'Now le's we watch ow' chanct an' git redd er dat p'ison-mean creetur whar done kilt ow' fren'. I boun' you w'en we git thu wid 'im dar ain' gwine be a piece uv "im lef" big 'nuff ter quile an onquile hitse'f.'

"So dey putt a watch on 'im an' las', one day, dey ketched 'im fas' asleep sumnin' hisse'f on a rock jes' outside de kyave an' one un 'em tucken him by de back er de neck, so's't he kain't bite an' de res' jump on 'im, an' dey mek shawt wu'k er dat sarpint: 'deed dey did. Dat sassified 'em fer a w'ile an' den dey 'gun ter spicion Canadi ag״in, 'kase onct dese yer 'spicions gits inter de haid, dey sticks closeter dan cockle-burs in sheep's wool, an' w'en hit comes ter gittin' 'em out, someb'dy mos' sho' ter git hurted in der feelin's, same 


\section{AT' TIIE BIG IIOUSE}

ez you git hurted in de fingers w'en you pulls out de cockle-bur's. Dey 'gun ter dog roun' an' watch 'im an' play 'im tricks. stealin f'um him an' eatin' up all de game he sont "em atter, "twel las" he see "twan't no good an' dat dey gwine do him harm ef he don' git shed ur 'cm; so he call 'cm all up an' he say, "Youtall is de meanes', hongries". 'ceitfules' creeturs on de face er de rearf, an' I gwine wash my han's ur you an' gin you yo walkin papers dis ve'y minnit. You kin quit my sarvice an you kain"t nurer git back in hit no mo", fer a fren' los" is a inimy foun', an' now we know whar we gwine stan' f'um dis time fo'th.'

"Wid dat he p"int his finger an" de wolfs dey know he mean jes' w'at he say, an" dey go slinkin' off thu de woods, snappin an' snarlin' at one nu'rr an' stoppin' now an' den ter howl back some sass at Canadi, an' w'ensomuver dey met, atter dat, dey wuz alluz inimies, jes" lak Canadi say "twuz gwine be; an 'f"um dat on de wolfs hatter fin' der own game an' Canadi hatter do his own killin'; an' sence den de wolfs ain' nuver bin no kind er use ter men, "kase dey is alluz 'ceitful an' cruel an' revingeful." 


\section{THE STORY OF A GIANT}

There was silence for a while in the little cabin, after the story of Canadi, during which Aunt Phrony sat gazing thoughtfully into the fire, having raked the cmbers together under the hickory log and blown them into flame. At last she said. giring rent to her thoughts of the past few minutes: "I reckon ef we cu'd jes" know all de curisome things an people an' creeturs whar bin on dis rearf an' quit hit fer good, we'd be dat "stonish" we ain "kin b"lieve ow" own senses. I yearn tell "bout de giants an' de 'li"l people" whar useter be, so"t I know folks wan't alluz de way dey is now. an' my daddy"s people dey tells lots er tales "bout creeturs whar useter live in de ol' days an' now is clean pe ish offen de face er de yearf. Seem lak ef all dem folls an dem creeturs cu'd come back, dey oon know hit fer de same place, an' dey'd be dat disapp"inted an' home-hongry de hull posse-cum-tat 'ud jes' lay down ağ in an' go back ter bones an' dus. I done rear so much bout $\mathrm{em}$ dat I kain't git 'em outen my haid. an' seem lak I kain't git my own cornsent ter leave dis worl' widout knowin' jes" who an' w' at bin yer befo' I camed. I mought ez well quit studyin' 'bout hit. dough. fer dar ain' no mo' chanct er knowin' dat dan dar is er knowin who an" w'at gwine come atter I'se gone."

"Did they really have people and animals then that we don't have now?" said Ned. 
The old woman answered with an emphatic nod that said more than words. and intimated that she knew far more on the subject than she was ever likely to tell.

"Please tell us about some of the old-time people," the boy urged, while the other two crowded close to her knees and looked up into her face with the interest they did not put into language.

"Le"s see," she said thoughtfully; "I b"lieve I 'member one "bout a giant. How does y'all think dat 'ud suit yo" notion?"’

Of course the children declared that a giant would be the very thing, so Aunt 'Phrony proceeded: "You know de Bible ses, 'Dar wuz giants in dem days,' so 'tain' no use fer no pusson ter say he don' b'lieve in giants; kase ef de Bible sez dar wuz giants, den dar wuz giants. 'Tain' say w'at days, but co'se hit mus' 'a bin de same days whar de Injuns knowed bout. But 'pears lak befo' all de giants wuz gone dar wuz some folks dat wuz small, lak we-all; so dat de worl' wuz kind er mix up, some big. some li'l. In dem days de folks built der houses raise' up th'ee er fo' feet f'um de groun' on blocks er wood. Dar wuz a man an' his wife had one'r dem houses, an' dey had a daughter whar wuz a mighty likely, pooty gal.

"Tell, suh, w'at do dat mis'able gal do but teck hit inter her haid ter ma'y a giant. De ol' folks dey 'suade an' dey 'suade 'er, but 'tain' no use; fer w'en a gal onct gits sot on de wrong man, de on'ies kyore is ter let her ma'y him an' fin' out her mistake, w'icht some time she do an' sometime she don', but w'ichurer 'tis, she ain' gwine let on. Dat's de gre't bizness in life wid lots er wimmins-not ter let on. Ef dey did, my gracious, 


\section{THE STORY OF A GIANT}

w'at sort er worl' 'ud dish yer be! To livin' in hit ef de wimmins onct turnt der tongues aloose 'bout der men-folks. De on "ies comfu't in de marter is dat nuttin' kin hol' 'em back f'um turnin' 'em aloose right at de mens w'en no one else roun' ter year 'em. Tongues wag all de harder 'kase dey bin hilt back de res' er de time." So Aunt 'Phrony, the victim of an unhappy marriage, rambled on to herself rather than to the children, whose presence she had almost forgotten in her moralizings on matrimony, in which she was prone to indulge whenever the suljject came up.

She brought herself back to her story with a jerk, saying: "Well, dat gal she done ma"y de giant, an' ef she wuz saw'y fer hit she ain' let on to her daddy an' her mammy. Dey live near her in one'r dem cur'ous li'l houses set up on blocks, an' er'y day she come ter see 'em an' pass de time er day wid 'em an' ax 'em how dey did, but dey nurer sot eyes on de giant any mo'n ef he wan't in de lan' er de livin'. Dey think dat sort er cur'ous, but dey ain' dast ter say nuttin' ter de gal, 'kase she done hol' her haid high an' look sort er fierce 'bout de eyes w'enuver dey try ter bring de talk roun' to'des him.

"Ery mawnin' w'en dey come outside de do', dar wuz a daid deer a-layin' on de groun', er mebbe hit 'ud be a daid tukkey all raidy fer cookin'. Dey 'mence ter think dish yer wuz a son-in-law wuf havin' atter all. but still dey wunner an' dey wunner dat dey ain' see him, an' dey sez ter one nu'rr: 'W'at time you reckon dat man git up ter go huntin'? 'Kase nemmine how early we git up we nuver kin ketch 'im.'

"All de same, dey et up de deers an' de tukkies, an' 


\section{AT TIIE BIG HOTSE}

den dey sot down an' dey studied an' dey studied 'bout der cur'ous big son-in-law "twel seem lak dey kain't git him outen der haids fer a mimnit.

"I'en der got outer wood de giant he'd fin' out 'bout hit, an' fus' news dey knowed he'd drag home a big tree f'um de woods, roots an' all, jes' ez easy ez ef 'twan't muttin' but a stick er wood, an' leave hit whar dey kin git hit. Yit dey nuver year no noise ner ketch him doin' de draggin : so dey kep' on wunnerin' an' wmmerin' an' studyin' an' studyin', but dey kep' on, all de same, burnin' de wood he bringed 'em. Sometimes dey sez, 'Tell, he ain' sech a bad son-in-law, atter all'; an' yit ag'in de ol' ooman she'd say: 'Jes' 'pears ter me I kain't stan' dish yer no longer. Hit gin me de creeps, deed hit do, ter know dat man whar so tall he hatter double up ter git in thu de do', an'so strong he kin drag an' pull trees, roots an' all, is a-dustin 'roun' ow' house in de night. an' hether an' yon thu de woods, fetchin' an' kyar'yin', an' we nurer seein' 'im any mo'n ef he wuz a sperrit. I don' kyare w'at come ner w'at go. jes' so I kin see hit, but w'en things git ter spookin' roun' in de dark, den I'se done fer.'

"De ol' man he say, sezee, 'Same way wid me, ol' ooman, but long'z he ain' did no harm yit an' done sare me a lot er elbergrease, I'se gwine keep my mouf shet a w'ile longer an' see w'at happen.'

"Things went on dat-a-way an' went on dat-a-way, de giant fetchin' de deers an' de tukkies an' de firerood, de ol' folks eatin' de game an' burnin' de rood an' wumnerin' an' wumnerin' an' studyin' an' studyin' 'bout de giant. Er'y day de gal come ter see 'em, an' 


\section{THE STORI OF A GIANT}

ev'y day befo" she come dey ses ter one nu'rr dat dey gwine ax her 'bout de giant, an' ev'y day w'en she git dar dey ain' do hit. Las', de gal have a li'l chil' an' she bring hit fer her daddy an' mammy ter see, an' dey mek gre't 'miration over hit, same'z de gran'dardies an' gran'mammies alluz does, an' de gal she look mighty please' an proud, same`z de young mammies alluz does w'en dey's slowin" off der babies.

"Atter de gal tucken de chil' home, de ol" ooman sez ter de ol' man: 'Dat sho' is a fine chil', hit sho' is, but I wanter ax you dis: is you year dat chil' let out de fus' smidgin ur a squeal? is you now? Fer ef you is, I ain'; not de ghos' ur a whimper, suh; not one. An' I have my 'pinion ur a baby whar ain' cry. Don' talk ter me 'bout good babies! Ef dey ain' cry, I knows dey ain' human. Sump'n cur'ous 'bout dat baby, an' dat gal kin hug hit up all she wanster, but ez fer me, I'se 'feard un hit, an' I ain' gwine tetch hit fer fear I git some kind er spell putt on me; 'deed I ain'.'

"De ol' man he say: 'Well, you know I ain' much on totin' babies, nohow; alluz pears ter me dey gwine drap ter pieces on my han's, an' I let you know I ain' gwine tetch dis un; seem lak I kain't feel dat hit's any a-kin er mine. Jes' lak you ses, sump'n cur'ous 'bout dat chil', an' I reckon we bes' go slow 'twel we fin' out w'at 'tis.'

"Dey kep' on gittin' skeerder an' skeerder an' de gal she kep' on bringin' de chil' ter see 'em ev'y mawnin'. Dey had mighty oneasy times tryin' ter be mannerly an' keep on de right side er de gal an' vit not tetch de chil'. Las' de gal see how 'twuz, an' she got hurted in her feelin's an' went home an' tol' de giant dat her 


\section{$\triangle T$ THE BIG HOLSE}

maw an' her paw wuz 'feard er de baby. Den de giant he got hurted in his feelin's an' co'se his feelin's wuz bigger'n her'n an' hurted him wusser, an' he jes' rave an' he kyare. He say: 'Ter I bin fetchin' game an' wood fer yo dadty an' mammy an' pomperin' 'em up 'twel dey ain' have nuttin' ter do, an' den dey ac' lak dis. Yer I wuz g'wine roun' dat house ev'y night an' cu'd jes' a knock hit orer wid my fis' same'z you smash a aig-shell, er blow hit down wid my bref; jes' one puff 'ud a sont hit orer in a jiff. But I ain' do nuttin' 't all 'ceptin' ter he’p 'em. an' yer dey hatter go an' git skeer'd er my chil". Dey is skeer'd er me, too; I knows dat; dat don' mek no diffens, but w'en hit come ter gittin' skeery 'bout dat po' li'l chil', I gwine gin 'em sump'n ter git skeery 'bout, sho' "nuff; I gwine show 'em huccome.

"Den de gal she saw'y she done tol" him 'bout her daddy an' her mammy, an' she 'suade an' 'suade him ter let 'em off, 'twel las' he promuss he let 'em 'lone, but he say: 'I tell you p'in'-blank dat ef I lets 'em off, I ain' gwine stay yer an' see 'em turn up der noses at my chil'; dat I ain`. I jes' grine teck him an' putt out f'um yer an' go so fur off dat dey ain' nurer gwine set eyes on him ag in : you kin jes' mek up yo' min' ter dat.' Den he rave an' he krave some mo' an' skeert de gal mos' outen her wits. IIer daddy an' her mammy yearn de soun' an' ses ter one nu'rr dat dar mus' be a thunderstorm gwine on up in de mountains.

"De gal she baig an' she baig, an' she say dat ef he teck de baby, she gwine go, too. Giant ain' sayin' nuttin', jes' toss his long hra'r an' stomp his gre't footses an' g'long 'bout his bizness. 


\section{THE STORI OF A GIANT}

"One day w"iles de gal wuz "way. de giant he tucken de baby an" putt out f"um clar tight"z he cu'd go. I" "en de gal got home an" fom" de baby gone. she knowed he done tucken hit, an' I tell you dar wuz some tall cuttin"up 'bout den. she tear her hyar an' cry an' scream an' go on 'twel de nabers come a-runnin' an" her paw an' her maw stoot dar lookin' on an' sayin': 'Th-huh! now you see dat! Ain' we tol' you so w'en you wanter ma'y dat good-fer-nuttin" giant? Mebbe, nex" time, you grine pay some "tention ter we-all."

"Gal went on cryin' an' wringin" her han's, an" las" she say she gwine git up an' foller de giant an' fin" him ef she kin. So she start off an" she go an "she go an' she go, follerin' de giant's tracks, fer dey wuz so monst'ous big der wuz mighty easy ter fin". She went thu de woods an' de fiel's an' up de hills an' down de hills, an' de wind in de pines kep' mo'nin'. 'Gone! gone!" an' de water in de branches call out orer de stones. 'Gone! gone!" an' de bu'ds chirp 'Gone! gone!' an' de squ'l up de tree drap a nut down at her an' squeak out 'Gone! gone!' an' er'thing keep hit up dat-a-way 'twel de ooman wuz plumb "stracted.

"Las', w'en she wuz clean beat out, wid her footses sore an' her han's all scratch" up an' bleedin' f"um de devil's-shoe-string. w"ich some folks calls hit de bamboobrier, she see de giant's tracks on one side de branch an' she look 'cross an' see dat 'tain' come out on tu'rr side, an'so she think he mus' be som'ers roun' dar. She look up de branch. an' sho' 'nuff, dar he wuz. settin' on a rock in the midse er de water wid de chil' in his arms. She ain' say nuttin'. jes' turn roun' an' steal thu de woods 'twel she come out right across f'um w'ar she done seed 


\section{AT THE BIG HOUSE}

de giant settin". Bless gracions! he wan't dar, not him. He knowed wat she wuz up ter jes" ez well ez ef some un done tol" 'im, so he tucken de chil' an' made one bigr step thu de water, swish! an sot down on nu'rr rock futher up. De gal she look up an see him; den she try de same trick ag"in. stealin up on him thu de woods, but he jes' showed all de toofs in his haid in a big grin an' shet 'em to wid a snap an' tucken anu'rr big step thu de water an' sot down on a gre't rock wid de water a-fussin' an' a-foamin' all roun' him an' de baby. Dat mek her fair' wil' an' she try ter fool 'im ag'in. But 'tain' so easy ter fool a giant, an' he kep' on tollin' her an' tollin' her up de stream 'twel she wuz nigh mos' daid an' hatter gin up de chase an" go back. All de way home she kep' wringin' her han's an "cryin' 'Gone! gone?" an' de wind an' de water an' de bu'ds in de tree-tops dey all call back at her 'Gone! gone!' lak dey felt mighty saw'y fer her.

"De nabers dey say it sarve her right fer not bein" sassified ter ma'y a man ur her own people. "Small man wan't good 'nuff fer 'er,' dey ses: 'hatter have big man. huh? Reckon she ain' g'wine have sech big notions de nex' time.'

"Gal she useter go out inter de woods er'y now an" den ter see ef she kin see any sign er de giant an' de habr, but er'y time she come back crrin' 'Gone! gone!' an' de wind an' de water an' de bu'ds arnser her back ev'y time, an' you kin year 'em sayin' hit down ontwel dis day ef you jes' stop an' stan' still an' lissen a w'ile." 


\section{WHY THE BUZZARD IS BALD}

Little Kit was fascinated by the story of the giant. possibly because it reminded him of his farorite story of Jack-the-giant-killer. He climbed up on Aunt 'Phrony's lap, put his little arms about her neck, and laying his soft, pink cheek against her dusky one, begged for "anuzzer giant story." Aunt 'Phrony's was not a rery responsive nature, but she could not resist the endearments of the pretty baby-boy. She hugged him fast, stroking his curls and declaring he should have what he wanted if she had to "bust her breens" to recall it for him. Her brains stood the strain, howerer, for she soon announced to the little boy, "Now, den, my lamb, my honey-bud, I gwine gin you a story 'bout de race 'twix' Mistah Deer an' Mistah Tukkey, an' w'ile 'tain' so much all bout a giant, yit dar's a giant comes inter hit.

"One time de Deer an" de Tukkey wuz gwine have a race. Mos" er de creeturs thought dish yer wuz plumb reedikelous, 'kase de Deer wuz knowed ez de swif'es' uv all de beaseses an' hit seem ter dem dat Tukkey wuz settin' hisse'f up might'ly ter race wid sech a runner ez w'at de Deer wuz knowed ter be. Dey ses, 'T'mumph! jes' look at dat feller's li'l haid an' you kin see w'y he talk foolish; ain't got room fer mo'n a thimbleful er breens. He dumno B f'um bull's foot. Struts 


\section{AT THE BIG HOUSE}

roun' an' swells hisse'f up an' looks mighty proud an' biggitty. but jes' let him open his mouf an' talk an' you kin look in thu de crack an' fin' out how empty his haid is inside.'

"But yit, atter all, de Tukkey wuz a right swif' bu'd in dem days, an'so w'en de creeturs all met toge'rr ter bet on de race dar wuz some un 'em w'at putt der bets on de Tukker."

"I thought betting was wrong," interrupted little Miss Janey, with her most knowing air.

"So tis, so "tis," said Aunt "Phrony. "Is you uver year me say 'twuz right? Hits plumb wrong, but de creeturs done hit, jes' the same ez men does; you kain't 'spec' dem ter be better dan w'at men is, dough I ain' so sho', atter all, but w'at dey is better. De beas'eses 'ud be 'shame' ter kyar' on de way some humans does, dat dey would. Anyhows, de creeturs sut'n'y bet on dat race. Ev'yb'dy wuz dar, an' some er de fowels betted on de Deer an' some er de beas'eses betted on de Tukkey, but mos' er de bu'ds wuz fer der own man, Mistah Tukkey, an' mos' er de beas'eses wuz fer Mistah Deer.

"Mistah Coon wuz struttin' roun', mighty empawtant, tryin' ter git folks ter bet wid 'im. 'I lay you five ter one on Mistah Deer; come on any un you dat ain' 'feard ter putt up,' sezee, 'I'se teckin' all de bets I kin git dis mawnin'.

“'Done!' sez de Owl, sezee, an' dey bofe went an' han'ed over der proputty ter de wimmin-folks dat wuz teckin' charge er all de stuff whar bin betted; dar wuz a gre't pile ur hit layin' on de groun' wid de wimmins keepin' gyard over hit. Lots er things changed han's dat day, I kin tell you. Even Mistah Ant done brung 


\section{WHY THE BLZZARD IS BALD}

sump'n ter putt up, an' 'twuz neener mo' ner less dan a daid caterpiller, dough you cu'd scacely tell hit fer dat, "kase he done pull out er'y single hya'r so's”t he cu'd drag hit down de hole whar he live."

"Pshaw! . Iunt Phrony," said Ned, "ants don't really do that, do they?"

"Well, I dunno wat-all ants does." she answered, "but I knows I saw some dat I wuz watchin' one day do dat ve'y thing. Dey brung Mistah Caterpillar ter de ant-hill an tried ter drag him down de hole wid all his fuzz on, an' w'en dey foun' dat oon do, dey jes' natchelly went ter w'k an' pulled ev'y hya'r out, an' w'ats mo' dey lef' 'em layin' in two straight lines on each side er de body, an den dey drug him down de hole. Dem ants is de smartes' creeturs in de worl', of dey is 'mongs' de li'les'.

"Well, now, 'bout dat race. Ev'ybd'y wuz dar, an' dar wuz a gran' talkin' an' buzzin' an' hummin' f'um ev'y sort er voice dat de creeturs talk in, an' dar wuz some fussin' an' quoilin'; dat sho' ter be wharuver any bettin' gwine on. Ol' Buzzu'd he wuz settin' up on a bare limb waitin' ter gin de w'u'd fer de race ter 'mence, 'kase he wuz 'p'inted fer ter be de jedge. Jes' den up come a giant ter watch de race, an' he year Mistah Lion gwine struttin' an' r'arin' 'bout, an' he lissen ter w'at he say.

"'Hi, yi!' sez de Lion, sezee, 'I ain' gwine putt up none er my propputty. Wats a li’l bit er truck lak dat in yon' pile, whar no pusson wants, atter all? I'se so sho' Mistah Deer gwine beat dat I'se willin' ter putt up my life 'gins' any fool dat 'll resk his life on dat numbskull, Mistah Wi'yum Wil'-tukkey. Reckon I orter 


\section{AT TIIE BIG IIOCSE}

know how fas" Mistah Dapple-deer kin go; I done chase him often 'nuff.'

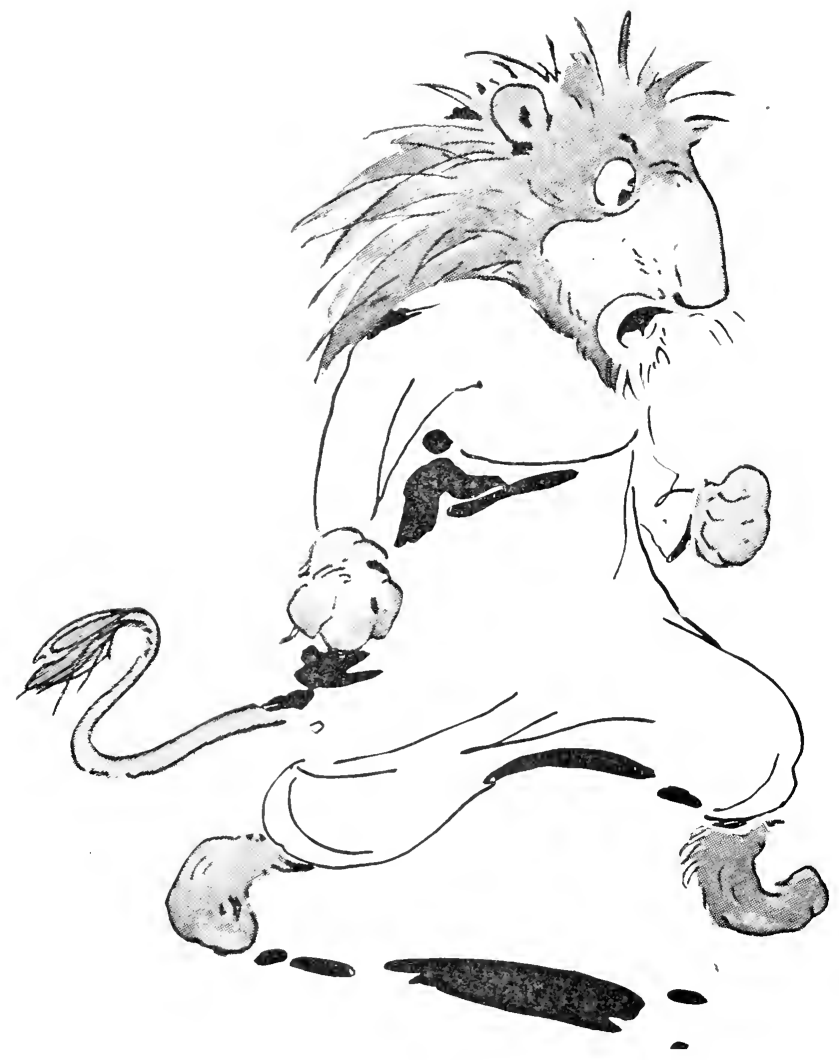

"Nob'dy spoke up, 'kase dey wuz all mighty'feard er Mistah Lion an' know dey ain' got much chanct w'en hit come ter tryin' der life 'gins' his. So ev'thing wuz 180 


\section{WHY THE BCZZIRD IS BALD}

mighty quiet all ter onct, an 'Lion he kep' on roarin' an' struttin' an' axin' some $m$ ter bet der life wid him. Jes' den 'long come a giant, one'r dese yer sho"'nuff giants whar got strenk nuff ter pull up a hill by de roots an walk off wid hit ef der wanster. He year w'at Lion sayin' an' he tuck up de bet. "I putts my life on de Tukkey,' sezee. 'I'se yo' man, Mistah Lion, an' I don' kyare who knows hit, dats me. I'll putt my life 'gins' de hull kit an' bilin', ef you sesso, an' not turn a hyar in de doin" sezee.

"Lion he gin a scawnful sniff dat wuz half snort an' half roar, an 'he sar. he do, "Well, ef dat don' beat bobtail! Dish yer human creetur ter set hise of up gin" me! Seem lak I sca'cely kin keep fum diggin' my claws inter him dish yer ve y minnit. Some er you fellers bes' come an' hol' me down 'twel de race is over, sezee. I let yer know dat none er de fellers 'cepted dat eenvite, dough; dey lef' him ter hissef. Walkin' up an' down, frailin' de air wid his tail an 'wu'kkin' his claws in an' out lak he 'z gittin' good an' raidy.

"Jes" bout den ol' Jerlge Bizzy Buzzu'd he gin de wu'd ter start. an' den Deer an' Tukkey wuz off lak shots, an' ev 'yb'dy craned der necks atter 'em an' fergot all 'bout de Lion an' de giant. At fus' Deer he wuz way in de lead, but 'long 'bout middle-ways er de co'se Tukkey he 'mence ter creep up on 'im, an' bless goodness, he kotch up an' kep' eren wid him an' jes' 'fo' dey got ter de een' ef he ain' come out a deer's lenk ahaid ur him. All dem whar bin bettin' on de Tukkey sont up a gre't cheer, an' Jedge Bizzy Buzzu'd he say, sezee, 'Tadiz an' gemmen. feller creeturs er dese diggin's, you done seed fit ter 'p'int me ter de pawtant office er jedge on dis 


\section{AT TIIE BIG IIOUSE}

'casion, w"ich office I done fill ter de bes' er my disabil'ty, an" I hope hit gwine be sassifact'ry ter you all w"en I 'nounce dat ow' fren', Mistah Wi'yum Wil'-tukkey is de winner er dis race, all fa'r an' squar', comin' out jes" one lenk ahaid ur ow' 'spected fren', Mistah Dappledeer. You is all now reques' ter step up ter de pile an' teck yo" goods whar you betted fer; de ladiz gwine see dat you git w'at comin' ter you. I don' wanter year 'bout none er you snougin' an' snatchin' w'at don' b'long to you. Le's be mannerly 'bout dis. You, Mistah Lion! You, Mistah Giant! Come 'way f'um dat pile! You got no bizness dar. Yo' 'greemint wuz ter bet yo' lifes on dis, an' we-all wanster see you come on an' fill de contrac'. I'se settin' yer ter see fa'r play an' I gwine do hit, ef I hatter be turn down at de nex' 'leckshun fer doin' hit. Dats wat a jedge is fer, ter see fa'r play, an' I wants y'all ter know hit. Dis way, Mistah Lion; dis way, Mistah Giant.'

"Den de giant he step fo"th an' he say, sezee, 'Well, Mistah Lion, dish yer's whar I reckon I gotter teck yo' life.'

"Lion he roar hisse'f hoarse an' bristle up his mane, - an' he say, sezee, 'Now lissen at dat fool man! He ain' nuver run up ag"in my ciaws er he oon talk lak dat. . Tes' wait ontwel I hook 'em inter him an' you 'll year him laugh on tu'r side ur his mouf. Who dat you gwine teck his life? Me? Now I stan's befo' all dese folks an' I axes you ter come on an' do hit.'

"Wid dat de giant he retch down an' pick up de Lion by de scruff er de neck an' dash him hard on de groun', an' w'en dey all come a-runnin', dar wuz de gre't Mistah Lion stretch out on de yearf. stone daid. Dey ail mek 


\section{WHY THE BLZZARD IS BALD}

gre 't 'miration, but dey ain' was'e many tears over 'im. 'kase dey wu all so 'feard ur him. dat mos' ur 'em wuz slad ter git shed ur him.

"Jedge Bizzy Buzzud he mek "em a li"l speech. IN say. seze. My fren's. ef I ain" stuck ter my p int an" done my jooty, dish yer rampin', rarin', rippin", roarin", boasin. tearin creetur and be livin dis re y minnit ter set yall shakin' an" quakin'. Dars no 'nyin' dat fac: I wants yall ter 'member dat nex' time rou has "casion ter "point anu re jedge. An' now I mus" come down offen dis perch an tell y"all "so long," "kase I sees some miglity p'tickler bizness waitin' fer me orer yonner in dat fur fiel',

"Wid dat Tedge Buzzu'd flopped his wings an' flewed off an' lef' de creeturs ter talk de marter over, an' I boun' rou der tongues wagged scannelous. De fowels whar better on Mistah Dapple-deer an' los' wuz plumb 'stunded wid de 'cision Jedge Buzzu'd mek in de case. 'Lawdy. lawdy!' dey sez, te think er dat ol' ninkum settin up ter know mo 'n we-all an' passin' jedgments fer us! We'll be switched ef we set down unner no sech ez dat. Tow see yer. wat we gwine do bout dis? Mistah Tarry-long Tarrpin. you orter be de one ter teck dat forel down, kase you done owe him a gredge urer sence de time he mek de 'cision 'gins' you w'en you race wid de hummin'bu'd. Mistah Lion he daid an' gone; he kain't sottle Jedge Buzzu'd's hash fer him, so now we gwine putt ow' 'pennance on you.'

"Tarr'pin he study a w'ile an' den he say, 'Gemmen, I wish you ain' spring dis on me so suddintly. Seem lak hit drive all de idees outen my haid. You orter tol' me 'bout dis sooner, den mobbe I moughter had 


\section{AT TIIE BIG IIOT'SE}

sump'n raidy. T'all have ter wait now ontwel I sees my way cle'r. Time. time, gimme time; seem lak I ain" nuver had "nuff er dat rit. But ef rou'll do dat, I'll promus ter do de bes" I kin, an' dats all I kien do.'

"Dey sez to "im. 'Teck all de time der is, Mistah Tarry-long. jes" so you fix him out in de een'. Git ro "se'f a good raidy an" den whu"l in an' teck him down so fur 't he"ll know jes' who he is.'

"Wid dat dey all up an' go home, an' f'um dat time on Tarry-long he study an' he study an' he study 'bout Jedge Bizzy Buzzu'd. Las' one day he come 'long an' seed ol Buzzu'd layin' fas' asleep in de heat er de day, teckin a noonin under a tree. He crope up closter an' he rear Buzzu'd snorin' 'way lak a steam injin' an' er'y now an' den lettin' out a li'l puff er a snort. 'De laws an' de prophits!' sezee, 'lissen at dat ol' man, 'nuff ter sturb de hull naberhoods. Temmine, suits my time prezackly.'

"He sot dar a w"ile laughin' at Jedge Buzzu'd, "kase he look plumb reedikelous wid his footses stickin" up in de air an" his mouf wide open. Den he crope up an" he crope up an' he try ol' Buzzu 'd by ticklin' 'im on de toes, but de Jedge wuz so fas' asleep dat he jes' drord 'em up a li'l an' gin a extry loud puff an' den went on 'tendin' ter his breathin". Den Tarrpin he crope up closter yit an' tuck a hull han'ful er li'l insec's an' putt emi on Buzzu'd's haid, an' den he crope off an' went home fas' ez he cu'd, w"ich "twan't no faster dan de law 'low.

"Jedge Buzzu'd sut'n'y had a time wid his haid. Dem norty li'l insec's ain' lef' him no peace day ner night, an' de long an' de shawt ur hit wuz dat he los' ev'y hya'r outen his haid. an' he bin gwine roun' bald-haided 


\section{WHY THE BCZZARD IS BALD}

urer sence. You kin see "im mos' any day in de 'ear perch' up in a ol' daid tree, wid his haid all bare ter de sun an' he wind an' de rain, lookin' mighty sollumcholly an' thinkin' over dem ol' days w'en he ac' de part er jedge fer de creeturs an' dey snatch him bald-haided fer his pains." 


\section{MIS' GOOSE DECEIVES MR. BEAR}

The children, not content with the society of Aunt Nancy in the morning, when she made their toilets. or in the evening, when she put them to bed, used to make her a visit now and then at her cabin. One morning they found her busy carrying in pumpkins from the little field behind her house. Ned thought it would be fun to kick them along like footballs, but Nancy said that pumpkins were made for eating, not for kicking. and that unless the children went about it in the right way she did not care about their help. Janey, with a shrewd eye to business, said they would all three turn to and help, if she would promise to tell them a story when the pumplins were all stored. The bargain was clinched, and four pairs of hands made short work of the pumpkins. Then they all sat dorn in the doorway of the little cabin, and Aunt Nancy said that the pumpkins had reminded her of a tale in which Mr. Bear got fooled by Mis' Goose.

"You chillen done yearn tell," she began. "dat gooses is kind er foolish, silly sort er fowels, an' I wanter tell you right yer an' now dat dey got heap mo' sense dan some er de folks whar calls names at 'em. an' ez fer de ganders, I'se yit ter see de human man teck de kyare uv his wife an' fambly dat dat ol' gander, yonner, do uv his'n. He he'p de goose ter buil' her nes', an' dey teck 


\section{MIIS' GOOSE DECEITES IIR. BEAR}

some er de down f'um der breastes an' line de nes', jes 'z wo 'm an' white an' pooty ez kin be, an' den she set on hit, an he keeps grard at he hen-house do' an' ef any pusson come near he beat his wings at 'em an' do his bes' ter drive em 'way. An'wen she go off ter eat an' res' husse ff he jos' matchelly squat down on de aigs an' keep cm wom ontwel she git back. Mebbe y'all has yearn de gnoses cry out in de night. Well, dat's wen dey change grard, fer dey sets a watch all thu de night an' w'enurer dey changes, de watchman gins out a cur'ous cry ter say dey's changin' grard an ev'ything gwine on all right. Yas. suh, gooses ain' de gooses folks bin allin' 'm all dese ears. An' I let you know dat big an' lumbersome cz Mistah Bar wuz, dar wuz a time w'en Mis' Croose got plumb de bes' uv “im.

"she wuz gwine warldlin" "long one day wid her chillen all strung out in a long line behime her. De goslin's wuz right roung an' she wuz teckin' 'em down ter de branch fer ter larn em ter swim. She wuz mighty proud ur 'em, an' she wuz gwine long wid her haid r'ared up, shooin' 'em ev'y now an' den ter keep 'em in de straight paf, an’ cacklin’ so`st ev’yb'dy cu'd year er: 'Tou Fluff! You Puff! You Buff! You year me? I want y'all ter keep in de paf. Yon's a dog; you better stick rlost ter me of you know wat good fer you!' So Fluff an' Puff an' Buff an' all de res' er de chillen dey turnt der toes in an' went waddlin' 'long in a straight line down ter de branch.

" 'Bout dat time dey met up wid ol' man B'ar. He wuz stayin' out in de woods all by his lonesome, an' w'en he see Mis' Goose gwine 'long wid all dat nice fambly ter keep her compny, he git strucken wid de 


\section{AT THE BIG HOUSE}

notion dat he want a fambly. too, ter teck roun' wid 'im an' chur him up an' drive 'way de low-downs. So he s'lute Mis' Goose mighty p'litely, tetchin' his paw ter his hat an' sayin'. 'Mawnin', Mis' Goose; mawnin', ma'am ; I hope I sees you well.'

"She gin a li'l hiss an' spread her wings an' done lak she gwine run at 'im, 'kase she wan't 'feard er no pusson, an' she mighty tetchy w'en she lookin' atter her chillen. But pres'n'y she see dat de ol' man wanter be fren'ly wid her, so she putt her wings down an' bob her haid, an' she say, she do, 'How you come on yo'se'f, Mistah B'ar?'

"He 'low, he do, dat he wuz kind er ailified, an' dat he feel de need ur a fambly ter keep him f'um gittin' lonesome. 'W'en I seed you comin' down de paf wid dat nice, big fambly er yo'n, Mis' Goose,' sezee, 'I ses ter myse'f, sez I, "Dat's de kyore I bin needin' fer dese yer low sperrits er mine, dough I ain' bin knowin' w'at 'twuz. I gwine ax her, dis re'y minnit, please ma'am, ter tell me how I kin git me a fambly lak her'n." 'Mis' Goose she turnt her haid roun' on de side an' cut one eye up at 'im, de way gooses does-'kase dey nuver seem ter look at you outen bofe eyes at onct-an' she see he mean w'at he say; so she mek arnser, she do, 'Well, suh, I done hatch out dese chillen f'um de aigs.'

"'Is dasso?' sezee. 'Wellum, I be might'ly 'bleeged ef you tell me whar I kin git me some aigs.'

"Right dar Mis' Goose wuz tucken wid a notion dat mek her langh an' langh on de eenside, so's she sca'cely cu'd stan' on one foot lak she bin doin'; she hatter putt bofe on de groun' so's't she kin stan' stiddy. But she ain' dyare laugh out loud, an' she nurer even smile 


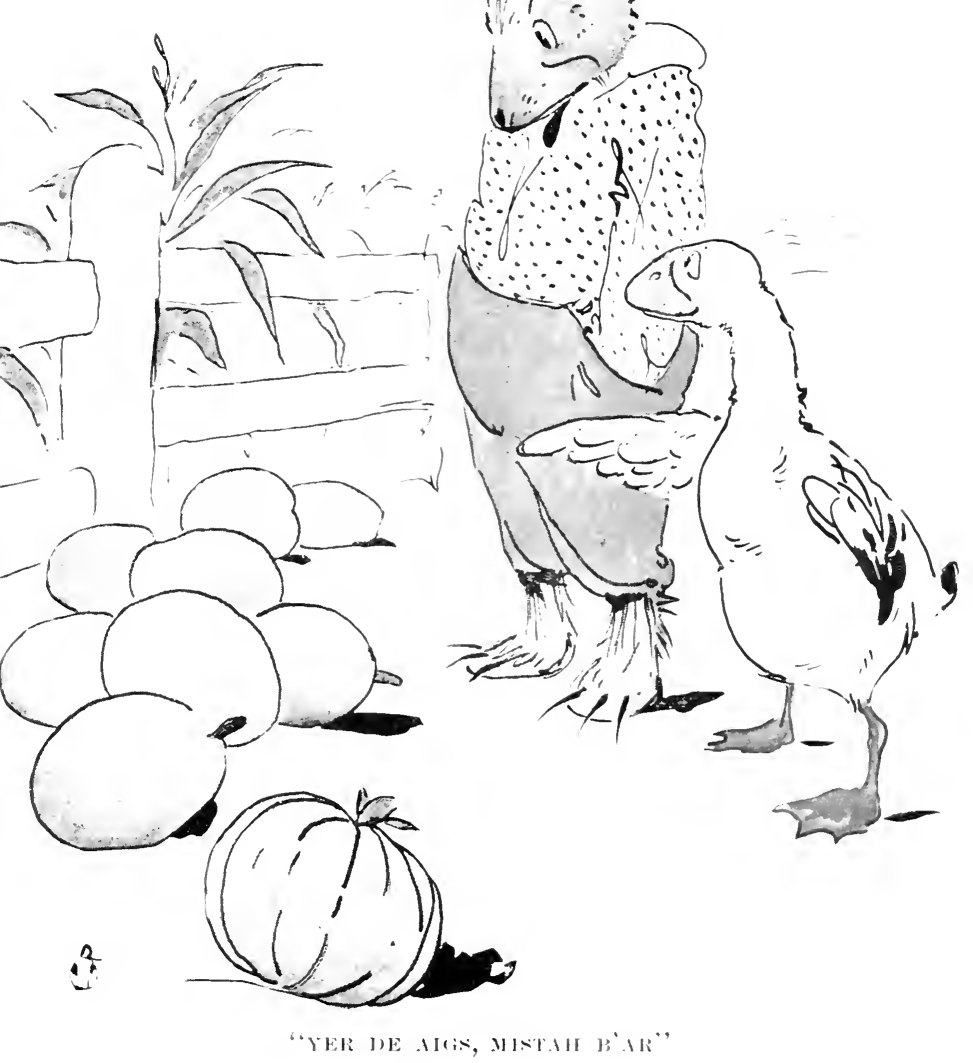





\section{MIS" GOOSE DECEITES MR. BE.TR}

wile she tell him: 'Lawdy! lawdy' Mistah B'ar, I swan ter man! I knows de vey contrapshun whar gwine suit. Over yon "in de fiel" is a hull ne: ful er aigs jes waitin' fer some un ter set on "em. I wish I'd bin able ter have mnertooken dat job myse f. but you see my han : right full a "raidy wid dere yer twelve li"l chillen er mine, an T'se feelin' right po"ly. too. f"um settin so long on de aigs. Done los" my flaish. suh. done los my thas - an my appentite ain wat "twuz. Ise bleegerl ter be hones" wid you. suh. an' let you know dat dish yer settin" hard wuk. suh. dat hit is.

"B"ar he ar. Bless ro" oul. Mis" Goose. I ain" look ter git me nuttin 'in dish yer -nfl"rin", dyin' worl" widout I w'k fer hit. an of a delikin lady lak you kin stan" hit. I reckon I kin. big an strong ez w' at I is.'

"Mis" Gonse she say. 'Well. come on den. ef you sesso,' an' she tuck an' led de way ter a fiel whar dar wuz a pile er pun kins in a fence cornder. an she say she do: 'Ter de aigs, Mistah Bar. Now. suh. lemme see you set on 'em. You gotter kirer "em er"y one wid yo" body an keep 'em nice an wo 'm, elee dey ain' gwine hatch out. an you have all yo trouble fer nuttin".

"ol" man Bar he squat down on de pun'kins an' he quile hise-f fu" dis-a-way an' den dat-a-way. an' dror his laigs up an" den putt em down. an' den git up an' tu'n clean roun an 'squat ag "in. Jes' w'en he think he'z all fix'. yer come a pun 'Kin rollin' out fus" one side an" den tu'rr. Wi:' frome the drin' ter langh. but she ain' dast ter. an "las" she whi"l in an" he p git him fix". Den she go off down de paf wid her fambly. laughin' so't she kain't speak. W'en se met up wir Mris" Molly Cottontail. Mis' Molly wait a li'l, an' den she say: 'You iṣ a 


\section{AT THE BIG HOLSE}

sho'-'nuff goose, sho' nuff! W'yn't you speak up lak you had some sense an' lemme know de joke?"

“Las' Jis' Goose tell 'er, an' she rell an' she holler an' hol' onter her sides. She ain' stop ter say good-by, but jes' went' stavin' long de paf 'twel she come ter de fonce cornder an' seed ol' B'ar. She let on she ain' know w'at he'z up to, an' she say, 'Heyo, Mistah B'ar, wat you doin' dar, suh, layin' in de fence cornder dis time er day?"

"He say: 'Jes' res”in' myse'f, Mis' Molly ; jes' res"in' myse'f. Done come a fur ways an' I'se tryin' ter ketch my wind ag"in.'

"She say, sez she, 'Dis a mighty fine day; I wish you come fer a walk wid me.'

"He say he too tired, an' den she say she wait 'twel he git res'ed. He baig her not ter teck so much trouble, an' she say 'twan't no trouble 't all, an' dey rum on lak lat, pow'ful p'lite, but all de time B'ar he wuz pestered ter know how he gwine git shed uv her, an' he wuz feard ery minnit dat a pun'kin wuz gwine roll out f'um beneaf him.

"All de time Molly Cotton-tail sca'ce kin keep her face straight, but las" she got tired er de fin an putt out fer home, tellin' him she be back to-morrer ter see ef he wuz res'ed up.

"Sho' 'nuff, back she camed, an" she say', sez she, 'Heyo, Mistah B'ar, I stopped 'long ter tell you I done foun' a tree up de road a mile er so, an' hit's dat chock full er honey dat 'twon't hol' nu'rr drap, an' ef you sesso, I'll go right 'Jong wid you an' show you de way.'

"B'ar he lick lis chops an' do lak he gwine git up. Den he 'member de pun'kins an' drap back, an' he say, 


\section{IIS' GOOSE DECEITES MR. BEAR}

he do: 'Thanky ma'am. Mis' Molly, thanky ma'am. I'se feelin' too painyfied ter go wid you dis mawnin', call roun' ag“in. ma'am, an' I go wir rou, sho". "kase rou knows I is 'tickler fond ur honey.'

"Inolly she hang roun', an' B'ar he try ter git her ter $g^{*}$ "long. but she tell him she ain pres" fer time. an" dat her ol man an' de chillens gwine keep "twel she git home. Ery day she come back an' ax him is he well "nuff ter go atter de honer, an' er"y day he say, he do, 'Tou mus" 'scuse me dis mawnin'. Mris' Molly, you sho' mus": de sperrit's willin' but de flaish mighty weak,. ma'am. mighty weak.'

"Las" she say, sez she, 'I b'liere you, suh; you sut'n'y has growed weak an thin an' pindlin'; you is nuttin' mo' dan de shadder er Mistah B'ar.'

"B'ar he gin a groan, kase he knowed twuz de trufe. He ain' bin off de nes" ter git him rittle. "Kase he 'feard de aigs mought git col'. an' he w'uz nigh 'bout starred. He ain' dast ter more. neener. fer fear de aigs roll f' um un'need. Hit 'pear ter him he kain't stan' hit ter keep still nu'rr minnit. Mis' Molly Cotton-tail knowed dat right well, but she kep' on naggin' an 'naggin' at him 'twel he say 'Drat de ooman, I wish I ain' nurer think 'bout gittin' me a fambly.'

"Las", one day, he kain't stan' hit no longer an' he riz up an' turnt hisse'f roun', but he wuz so weak dat he jes' fell back onter de pun kins, an' some ur 'em roll out f"um un'need an" de res" wuz all squehed up, "kase dey'd 'menced ter git sof' by dat time. B'ar he groan an' he moan an' he cry an' he roll on de groun' an' dig bofe fistes in his eyes. 'Bout den Mis' Molly come 'Iong an' year de gwines-on, an' she stop an' hang orer de fence 


\section{AT THE BIG HOLSE}

'tendin' lak she mighty saw'y. She say, sez she, 'Lawsy, Mistah B'ar, w'at does I see, suh; an' w'at is de 'casion ur all dis mis ry?"

"O lawd! O lawd!" sezee, 'my po' fambly; my po' fambly!" an' wid that he bus' out ter cryin' ag“in. W'en he git so'st he kin talk, he say: 'Mis' Molly Cotton-tail, I ax you, ma'am. fer ter look at dem aigs whar I bin settin' on all dis time, an' whar I 'lowed I gwine hatch out inter a nice li'l fambly. You sees fer ro"se'f how mos' ur 'em all sqush' up. an' de res' done roll 'way f'um me an' git stone col'. An' rer me all hongry an' thu'sty an' wo" ter de bone, all fer nuttin'?

"At dat he cry mo" louder dan befo". Den Mis' Molly ain' try ter keep in no longer. She lean up ag"in de fence an' she rell an' she holler. 'Hey, lawd!" sez she, 'he call dem aigs! dem big ol' rotten pun'kins is aigs! Well, live an' l'arn, sez I! Ter me bin 'lowin' all dese cars dat dem things wuz pun'kins, an' now I fine dey is aigs. Ef I'd a-knowed w'at you wuz up ter all dis time. suh. I'd a-bringed you some er dat honey whar I bin tellin' you 'bout. 'kase you mus' be mighty hongry by dis time. Dish yer settin' bizness is mighty tryin'. I year tell, so tryin' dat de mens mos' in gin'ly leaves hit ter de ladiz. Lemme know nex' time you gwine hatch out a fambly, an' I try ter hep you out in de marter er vittles. You'se a plumb skillintin; you sho" is!"

"Ol' B'ar gittin' lind er rile up by dat time, an' do lak he gwine teck atter her. She lit out f'um dat, but she see he wuz too weak ter foller her, so she run back fer a minnit an' stuck her haid thu de fence, an' sez she, kind er rollin' one eve on de pun'kins an' one on 


\section{MIS' GOOSE DECEITES MIR. BEAR}

him: 'Tm-umph! Mistah B'ar. dis sut'n'y is a pity. 'kase ef dem aigs had on' hatch out. yo' fambly' moughter bin some pun'line. sho' 'nuff.'," 


\section{MR. BEAR TENDS STORE FOR MIR. FOX}

"Aunt Nancy," said Ned, after the story of the bear and the pumpkins, "I wish you'd tell me what relation Molly Cotton-tail was to Mr. Hare."

"Relation?" she asked; "you mean w'at a-kin wuz she? To kin' 't all. 'scusin' by ma"iage; "kase she wuz his wife, chil'; an' atter she live wid him so long time, she got ez trickish ez w'at he wuz, an' dat's sayin' a heap. She wuz a mighty smart ooman, an' she knowed how ter read an' write, an', w'at's mo', she cu'd mummick de writin' er mos' any pusson she wanter, an' dat come nigh gittin' her inter a hull passel er trouble onct, but she wuz so slick she wiggle ont jes' in time. Hit wuz lak dis: Fox he done tried his han' at huntin' an' fishin' an' farmin' widont mekin' much ur a fist at any un 'em, an' las' he set him up a li'l sto' at de crossroads; one'r dese yer sto's whar de folks brings a li'l haid-turn er truck an` trades hit fer bacon an’ meal.

"One day Mis' Molly Cotton-tail, she done rumned outer sump'n ter eat, an' de chillen wuz hongry an" baiggin' fer vittles. She say to husse'f: 'Dese chillen gwine run me 'stracted. I hatter stay home yer an' lissen at all der bodderment w'iles der paw goes carawtin' roun' de kyountry enj'rin' hisse'f might'ly. 'Tain' fair. But nemmine I gotter feed dese chillen, an' I knows w'at I gwine do.' 


\section{MR. BEAR TENDS STORE FOR MIR. FOA}

"Wid dat she sot down an' tucken a piece er paper an' a ink-bottle an' a quill, an' she stuck de quill behime one rear an' sot dar studyin' an' rumnin' her fingers thu her hyar 'twel she got de marter all fix up in her min". Den sle tucken de quill an' wit a order ter Mistah Fox fer a bag er meal an a shoulder er meat, an' she tuck an' signed Mis' Fox's name to hit. jes' 'zackly de way she done seed Mis' Fox wite her name one time. Den she sot down in de to an' wait fer some pusson ter pass by. Fus news you know, yer come Mistah Bar amblin down de road. By dat time he fergit how Mis" Molly done laugh at him "bout de pun"kins, an' w'en she mek her manners to him, nice an' proper, he arnser mighty p'litely. She ain' knowin' how he gwine treat er, but w'en she see 'twuz all right she 'mence muchin' him, 'kase she wuz a gre't han' ter flatter folks, an' wen she git things w'k up ter de proper p"int, she say: "'scuse me, Mistah B'ar, I is "sentially a backwu'd ooman an' I hates ter as farers, but my chillen is hongry an' no rittles in de house an' der paw f'um home. I be might ly 'bleeged. suh, ef rou leare dis order fer me at de cross-roads sto' an' bring me de vittles on yo" way back.'

"B'ar he say "tron"t be no trouble, an" he tucken de order an' lef' hit at de sto' an' den got de bacon an' meal on de way back an' fetched hit ter Mis' Molly. 'Twan't long 'fo' 'twuz all et up. an' 'twan't long. neener, 'fo' Wistah Fox fin' out de vittles wuz lef' wid Mis' Molly stidder wid his own ol' ooman, an' he 'clar' he gwine git even wid 'er fer forgin' his ol' ooman's name.

"Mis' Hrar' she ain' knowin' he fomn' her out, so one day she go inter de sto' ter trade, biggitty ez you please, 


\section{AT 'TIIE BIG HOC'SE}

an' he up an' ax 'er fer ter keep sto' a minnit w'iles he step ont. She kind er smell a mouse. an" she tell him she ain' got time fer ter tarry. Den he tucken her by de scruff er de neck an' tie her up good an tight, an' he say. sezee: 'Ch-huh! Forge my ol' ooman's name, will you? Eat up my meal and bacon, hey? Trash er de worl'! I gwine go out an' git me a cowhide an' gin you de bes' larrupin' you uver has had er uver is gwine ter git.'

"He went out an' lef" 'er dar studyin' 'bout de fix she wuz in, an mos' pussons 'ud a felt skeerder an' skeerder ev'y minnit, but, bless yo' soul, Mis" Hyar' wan't faze' by hit. She start in ter hummin' one'r dese gaily ol' darnsin' chunes an' pattin' wid her footses. 'kase her han's wuz tied behime her. Las' she bus' out at de top ur her roice inter de wu'ds er de song:

\section{'Sam, Sam u'uz a funny ol' man, \\ Fried his meat in a fryin' pan, \\ Combed his haid wid a wagon-wheel, \\ Died wid de toofache in his heel.'}

"'Bout dis time some un come sa'nterin' down de road an' year de noise an 'poke his haid in de do.' Who shu'd dat be but Mistah B'ar, an' he say, sezee, 'Hey. Mis' Molly Cotton-tail. wat de meanin' er all dis racket, an' w'at you doin' all snarl' up in dat rope?'

" "Well,' she 'low, 'I come yer ter git some colamel fer my sick chil', an' Mistah Sly-fox he up an' tell me he gwine gin a party at his house to-night, an' he ax me fer ter stay an' jine in de fun, an' I 'low I kain't, 'kase I hatter git back ter my chil', an' he 'low dat dey 


\section{IR. BEAR TEXDS STORE FOR MR. FOA}

kain't git "Inng widnut my comp'ny nolows an' dat he b'lieve I'se too stuck up ter 'sociate wid his fambly an' jes" mek up dat tale bout de sickness er my chil". I kep" on tellin" him I kain ‘t stay, an las" I say I go home an' teck de colamel wid me an' see how de chil" is. an" come back. But he "low, he did, dat a bud in de han" wuz wuf two in de bush. so he tucken de rope an" tie me up dis-a-may twel night. Den he step out ter mek come reddrments fer de party, an he ax me fer ter mind de sto" wiles he"s gone. Ter I is, settin yer tryin" ter keep my min off my po sick chil by singin an knockin' time. You ain nurer bin a mammy. Mistah Bar, er you d know jes" how I'm feelin" dis re minnit." an" right dar she let two big tears fall down on de flo". ker-splash!

"Bar felt mighty saw"y wen he see dat: mens is right easy tucken in by a few li'l ol' tears, an' he say dat he ontie her, ef she say de wu'd, an' let her go. She say, 'Yas, suh, but Mistah Fox he done 'spec' me ter see dat nuttin' git stole outer de sto'; w'at I gwine do 'bout dat?'

"B'ar he 'low dat he ain' min' tendin' sto' a li'l, an' she tell him, 'Go ahaid. den, an' ontie me.' Den she say' he better let her tie him up in de same place so's't he kain't change his min' 'bout stayin' fer de party. Bar say he wan't hankerin' atter any gay doin's, but ef dar wuz any vittles ter be 'stroved at de party. he wuz de man fer de place. So he let Mis' Molly tie him up an' den she went clippin' down de road, stoppin' at de turn long 'nuff ter sing out: 'O Mistah B'ar! O Mistah Bar, I hope you enj'y yo'se'f at de party! Dey tell ne hit gwine be mighty small an' s'lect. 'Tain' s'prise 19 ร 


\section{AT TIIE BIG IIOCSE}

me of you hatter do mos" er de Rarnsin yosef.' Wid dat she went a-kitin', an' den she sip inter de bresh an' double an' come back an suput down by de sto’ ter lis:ectl.

"Pres'n y yer come Mistah Fox lopin' back wid a grete cowhide in his han', an' he wuz mo'n s'prise' wen he see ol' B'ar all squoge up an' tied fas' in de place whar he done lef' Mis' Molly Cotton-tail. 'Laws-amuss!' sezee, "wat in de name er de ring-tail-roarers is de meanin er dish yer picce or bizness? Who done tie you up dis-a-way? Dat Molly Hyar', I be boun"?

"De same, sez de B'ar, suee.

"Huceome dat?' sez de Fox, sezee.

" Trell, suh,' Mistah Bar say, 'she tell me you done tie her up ter mek her stay fer yo party to-night, an' she say she natchelly pinin' ter git home ter her sick young un, an' she cry a li'l an baig a li"l, an' las' I tell her I jes' 'z lief teck her place an' tend de sto' an' go ter de party.'

"Fox he curl his mufstarsh an' twis" de een' up an' look at ol' B'ar outen de comder ur his eye an' he say: 'Th-hulh, is dasso?' You tol' her dat. did you? You willin' ter stay ter de party? Well, ef dat de case, kin you tell me w'y she hatter go ter wu'k an' do you up in all dem hard knots?"

"B'ar he say: 'Dat's all right. I turn her a-loose, an' den she tie me up dis-a-way 'kase she laugh an' say I mought change my min' 'bout stayin' fer de party, so she bes' mek sho' ur me. fer you so hard up fer fren's 'mongs' de creeturs dat you hatter do dis-away ter git anybidy ter come.'

"Dat mek Fox madder'n a hatter, dough he ain' so 


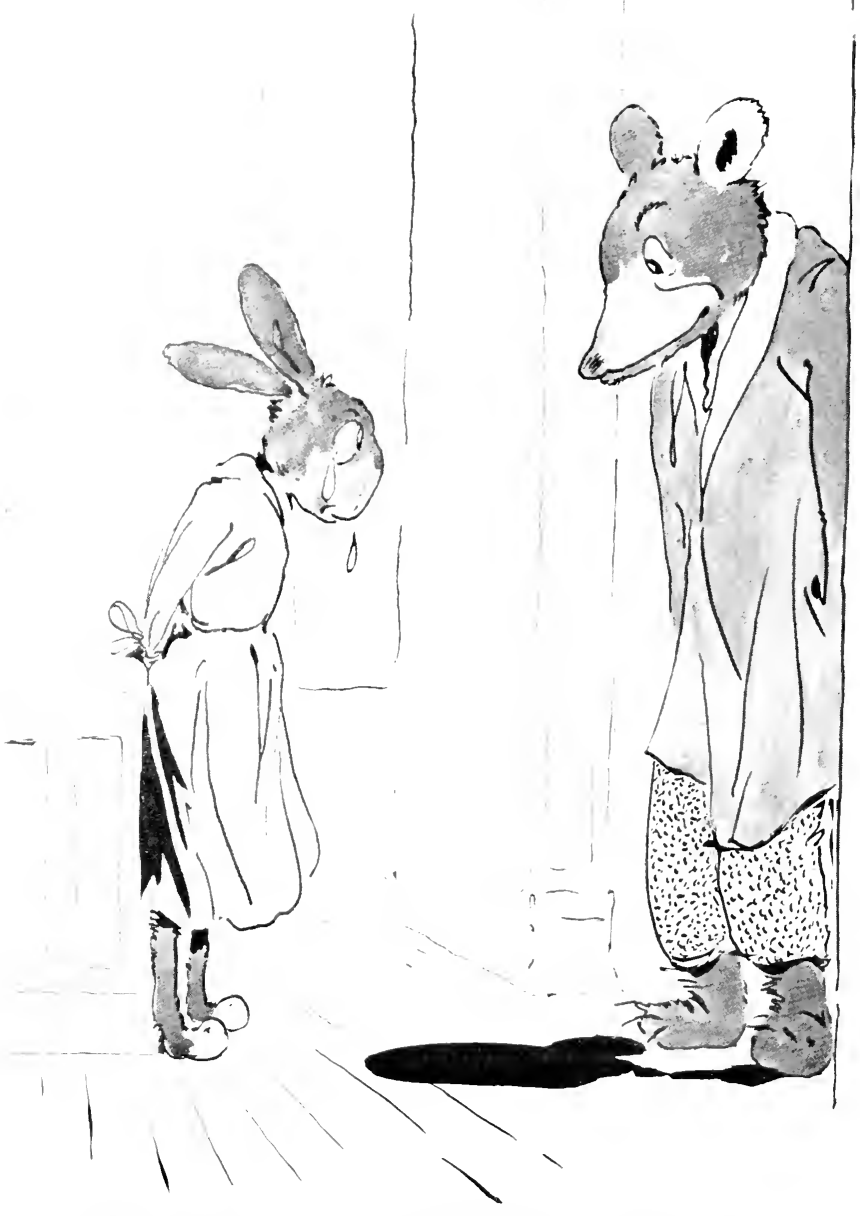

MIS' MOLLY LET TWO BIG TEARS FALL KER-SPLASH 



\section{MR. BEAR TENDS STORE FOR IIR. FOX}

mad but w'at he kin laugh an' holler an' slap his han' on his knee. 'Lawd! lawd!' sezee, 'ef dat ain' one smart ooman! Well, sulh, de on'ies party I wuz gwineter gin wuz a hidin'-party, an' me an' her an' dish yer cowhide wuz de on ies folks eenvited. an' now, suh, sence you done let de chief mo'ner go, w'y dish yer gwine be yo' chanct ter do de cryin'?

"Wid dat he whu'l de ol' rawhide roun' thu de air 'twel she snap lak a snappin'-turkle, an' bring her down on Mistah B'ar's back, zip! zip! zim! Bar he rared an' he charged an' he tore an' he swore an' ha growled an' he howled, but 'twan't no use, Mis' Molly she done tied him up good an' fas'. Fox he done whup him an' whup him 'twel de hide hung offen him in plumb ribands. Fox kep' on 'twel he got him right much skunt up, an' all de time he wuz jawin' him wid ev'y lick. 'I gwine l'arn you some sense, you gret big lan-lubber! Gwine l'arn you not ter be tucken in by ev'y li'l ol' ooman whar kin pump de water outen her eyes in thee shakes ur a sheep s tail! Gwine larn you not ter meddle in urr folkses mixes. You is de re' $y$ man whar comed yer an' bringed me dat order f'um my ol' ooman whar nuver come f'um her 't all, an' 'twuz you toted off de meal an' bacon ter ol' Molly Cotton-tail. Ain' you know dat ooman well nuff by dis time ter know dat you gwine git in trouble ef you don' keep 'way f'um her? You ol' 'nuff ter know better, 'deed you is; anyhow I gwine l'arn you.'

"Ol' B'ar he git ter cryin', an' ev'y time de whip come down he let out a big 'Boo-hoo!' Nis' Molly she wuz settin' outside lis'nin', an' ev'y time he say 'Boo-hoo!' she laugh 'Ho-ho!' at de same minnit, so dey ain' year 


\section{AT TILE BIG HOUSE}

her 't all. I'on Fox turnt Mistah B'ar a-loose, she wuz nff outen dat in a jiffy, an B'ar ain" see her fer one long spell. He hatter stay home an' nus his hide for a w'ile, an' he vow ter goodness dat he gwine frail Molly good nex' time he meet up wid her. "Mis'able li"l fippennybit,' sezee, 'her ter git a gre thig man lak me a th'ashin' f"um dat ol' S'lickrr Sly-fox! I knock em bofe inter de middle er nex' week nex' time I ketch 'em, dog me ef I don?'

"Las', one day, Molly seed him comin' down de road, an' she turnt off inter de bresh an' scooted thu a shawt cut 'twel she got way beyont him. Den she hop inter de middle er de road an' sing out, 'Heyo, Nistah B'ar. how you enjy yo 'se'f at Wistah Fox's party? I done rearn dat you sing mo' louder an’ jump mo`higher dan any urr man at de doin's. To pusson nd think dat jes' ter look at you gwine 'long'so sollum an' stiddy. You kain't tell, dough, mens is mighty 'ceivin': 'tain' safe fer us po' wimmins ter place ow' 'pennance on yo' looks.' Wid dat she gin her behime laigs a flirt in de air an'

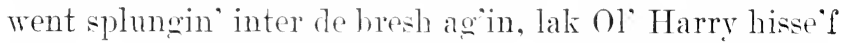
wuz atter her. w'ich mebbe he wuz, an' puttin her up ter all her mischief." 


\section{A STAR STORY}

One evening the dhildren encountered Aunt Phrony sitting in the doorway of the cook-house. gazing intently at the star's which twinkled orerhead like so many huge fireflies. She was of alsorber that she lid not seem to notice them until Janey softly twitched her apron and begged to know what whe was looking at.

"Lookin" at de stars," hle said.

"What for " "aked taner. who was alwars as full of questions as the proverbial "Iankee."

"Kase I wanter," said Annt Phrony.

"What makes you want to?" persisted the relentless Janey.

"IT"at mek you wanter ax me so many queschins: kin you tell me dat?" said the old woman. suddenly turning the tables on Miss Janey.

"'Canse I want to know," answered the irrepressible little questioner.

The old woman heaved a sigh of resignation as she sairl: "Well. I spose I mought ez well kyave in fur" ez las'. 'fo' I git my life jes" natchelly pestered nuten me. I wuz settin' yer lookin' at de stars, tryin" ter pick out de ones whar my daddy useter tell me tales 'bout, mo' 'special de seven li'l stars whar he say wuz onct weren li'l Injun boys." Here she pointed out to the children the cluster of stars known as the Pleiades and showed 


\section{AT THE BIG HOUSE}

them how six of the stars were very close together indeed, while the serenth was a little remored from the rest. This she explained by saying that one was the roungest boy. who had lagged behind, not being quite able to keep up with the others.

"De tale runs on lak dis," she said: "Dese yer seven li'l bors wuz sort er scampish li'l chaps, all time runnin' an' playin', no notion 't all er mekin' derse'fs useful, sump'n de way some chillen is dese days. Dey all un 'em had bows an' arrers an' dey wuz mighty good shots, dat dey wuz. Dey useter practuss shootin' at marks an' th'owin' things up inter de air an' shootin' 'em 'fo' dey fall to de groun'. Dey had one game dey wuz pow'ful fond er playin': dey wuz at hit mawnin', noon an' night, an' 'twuz lak dis: One un 'em 'ud th'ow a cawn-stalk up in de air, an' dey'd all shoot der arrers at hit, an' w'ichurer come de clostes' 'ud git all de arrers dat bin shot. Shootin' fer keeps, dey wuz.

"Dey live wid der ol' gran'mammy, an' she wuz too ol' ter do much wu 'k, an' dar wan't no men-folks in de house ter shoot game fer her, so de vittles wuz right sca'ce sometimes. Dem mis'able li'l boys, ef dey'd a-turnt der arrers a-loose at de deer an' de bu'ds an' sech ez dat, stidder at no-kyount cawn-stalks, dey moughter he"p der granny out an" gin her all de game she want. Not dem! ttwuz shoot, shoot all day long at de cawn-stalks stidder at w' at run in de woods. W'en night come dey'd march home jes' ez biggitty ez you please an' hongry ez houn's an' 'spec' der granny ter feed 'em same ez dey bin wu'kkin' hard all day.

"'Las' de ol' ooman git sort er outdone, an' she say, 'I know w'at I gwine do ter fix dese chillen off an' l'arn 202 


\section{A STAR STORY}

'em a lesson. De on'ies thing dey gwine git f'um me dis night is cawn-stalk soup: dey lak cawn-stalks so well dat I gwine gin 'em der fill fer onct.' So she ga'rrd a lot er de ol' stalks an' put 'em on an' b'iled 'em, an' w'en de boys come traipsin' in, hongry ez wolfs, she putt de new soup befo' ' $\mathrm{em}$. Dey dabbed in in a mighty hurry an' tuck big mouffuls, an' den sech mekin' faces an" sech spittin an' sputterin' you nurer seed. De fus' li'l boy he clap his han' ter his mouf an' he say, 'M!" Second li'l boy say, 'Hm!" Thu'd li'l boy say, "Whew!" Fo"th li'l boy say, 'Oo-0o-oo!' Fif' li'l boy say, 'Pf!' Sixt' li'l boy say, 'Bf!' Serent' li'l boy say nuttîn' 't all, "kase his mouf all scalted an' he kain't.

“"W'ats de marter?' sez der granny, sez she, 'ain' y'all lak dat cawn-stalk stchew? 'Tain' poss'bl' you kin git too much er cawn-stalks, don' tell me dat, 'kase I ain' gwine b'lieve hit. Saw'y I kain't gin you any dese urr vittles. Dish yer stchew gwine be yo' stay an' stan'by 'twel you quit yo' foolin' an' set ter wu'k an' bring me home some game. Long 'fo' yo' daddy wuz ol' ez you-all he useter bring me home bu'ds an' squ'ls an' hyar's. I dunno w'at de marter wid chillen dese days; seem lak dey gittin' mo' an 'mo' trouble an' less an' less use ter de growd-ups. I wunner ef de time gwine come w'en you git too lazy ter eat? Naw, sul, I don' reckon dat'll uver happen in my day. W'ats de good er me stuffin' an' stuffin' y'all jes' ter mek you grow up inter lazy, wufless men-folks: an' ef a Injum man kain't hunt, I ax you w'at is he good fer, den? He let de wimmin folk do er'ything but dat, an' now yer you wants ter mek me tu'n in an' do dat, too.'

“De li'l boys ain' say nair' w'd, jes' picked up der 


\section{AT TIIE BIG IIOUSE}

bows an arrers an' putt out f"um de louse tight ez dey kin go, de ohleses' boy in de lead, an' las' ur all de youngeses hoy. whar wuz li'l an' shawt an' fat, an' kain't run fas" ez tu'rs.

" 'Now, w'at in de name er goolness tecks dem chillen outen de house at dis time er night, sez granny, sez she. "Whar is dey grine an" w'at dey gwine do? Come back yer, chillen! Come back yer, 'fo' I teck a stick an' go atter you!' She go ter de do' an' call an' call an' call 'em, loud ez she kin, but dey ain' pay no 'tention 't all, jes' let on dey ain' year her an' run all de faster.

" "Mussy me!" she say, 'has I gotter pick up my ol' bones an' try ter ketch dem chillen? Come back yer, youall, ef you know wat bes" fer ro hides. Ef I hatter come dar I tan you black an’ blue. I'all bin sp ilin' fer a good frailin' dis long time, an' ef I does hatter come dar, I'll walk up an' down yo' kyarkiss a time er two an' gin you sech a lambastin' dat even de dogs in dis naberhoods ain' gwine know you.'

"Chillen ain' pay no 'tention, so de ol' ooman she tucken a long bref an' ga 'rred a long switch an' putt out atter de boys. All ter onct, wile she wuz chasin' 'em she seed 'em 'mence ter riz up f'um de groun', an' she stop shawt an' open her eyes an' mouf wide an' hol' up her han's at de sight an' call out fer 'em ter stop. But bless goodncss, dey kain't stop, an' w'ats mo', dey 'gum ter whu'l roun' in de air w'iles dey went up, an' dey whu'l faster an' faster 'twel hit mek der oì' granny plumb dizzy ter look at 'em. Dey cried an' dey hilt out der han's ter der granny, but 'twan't no use; she cu'dden he'p 'em. Dey whizzed so fas' dat dey jes' look lak black specks ez dey went up. Dey go up, up, up, whu'lin' an' 204 


\section{A STAR STORY}

whu'lin' an' whu'lin'. 'twel las" dey lit ker-smack ag"in de sky, an' dey wuz gwine so hard dat dey jes" stuck fas". an' dar dey bin wrer sence. Dey kain't git a-loose; dar" dey is pris'ners. all bekase dey wo sasey an' ugly an' ain' min' der granny.

"De po' ol" ooman ain" nurer see 'em no mo"; sle live on all "lone in de house. an" w"en de nights wuz cle"r she"d set in de do" an" try ter mek ont de seren li"l stars whar she knowed wuz de norty li'l ehillen. She'd set dar an rock back an' fo"th an look up at de stars an' say. 'Po' li'l chillen, po' li'l fellers, wunner will dey urer come back ter de yearf ag"in. Ef dey wuz yer riglit now I boun' you I'd gin 'em one good feed an' no queschins axed. neener.'

"But dey nurer come back no mo". Fer a long time nob'dy know w'at become ur em, but las' de ol' ooman kain't keep de sekert no longer, an' she call de nabers' chillen roun' her an' p'int out de seren stars an' tell 'em jes" how hit happen.

"So all de li"l chillen tucken ter watchin" dem stars w'en de nights wuz cle'r 'nuff. W'en dey see one'r de seren gin a twinkle dey"d say "twnz kase he jes' shot a arrer outen his bow. An' de ol ooman died. an' de li'l naber chillen growed up an' died. an' der chillen died, too. an'so on an so on down ontwel dis time, yit dem li'l Injum boys is still stuck fas' in de sky.

"Ef you"ll look up dar now you kin see 'em. all elost toge'rr. 'scusin' de youngeses' one; he so li'l an' his laigs so shawt dat he got lef' behime de res', and dat huccome he ain' right wid 'em. Ef you look sharp you kin see 'em shootin' a arrer now an' den; you'll know dat by de twinkle. 


\section{AT THE BIG HOLSE}

"Dish yer tale orter l'arn you dat sump'n sho' ter happen ter chillen whar is lazy an' cross an' sassy ter de growed-ups. Dey boun' ter git stuck fas' som ers, dough mebbe "twon't be in de sky. I has knowed sech chillen ter git fasten ter de baidpost er lock" up in de closet. "Tain so fur awars. but hit seem ter arnser de puppus," and here she looked meaningly at "Wi-yum," who was in disgrace for having run off and staved all day at the cross-roads store without leare.

She rolled her eyes up toward the stars again and said musingly, "I lak ter know how many er dem ol' tales 'bout de stars is true, er ef dey ain' true, who 'twuz dat fus' got up de stories. Mus' a bin long, long time ago, for no pusson seem ter year nuttin' lak dat dese davs."

The children asked if there were other stories about the stars.

" 'Deed, dat dey is," she answered. "Daddy knowed a heap 'bout de mawnin' star an' de erenin' star an' a lot ur urrs, but hit bin so long sence I year "em dat I kain't rightly call 'em ter min'. I don 'spec' I'd 'a'membered dis un ef Wi-gum's grines on ain' putt me in de min' ur hit. Seem lak you kain't git so far back in de ol' times but w'at you year tell 'bout norty chillen, jes' de same ez in dis day an' gineration; de crap nurer fails ner gins out, w'aturer else gits sca'ce." 


\section{WHY CRABS WALK BACKWARD}

One morning the children were playing out in a cold, drizzling rain, and Aunt Naney had several times adrised them to come in without effect.

"I"all gwine ketch ro" deaf"," she said, "an' den whar'll you be? Don' come 'roun' me wid yo' aches an' yo' pains an' yo' sniffin' an' barkin', 'kase I ain' gwine teck up my time wid chillen whar dunno 'nuff ter come in outen de rain; "tain wuff my w"iles."

"It isn't raining much; we won't take cold," they protested. but Aunt Nancy continued her harangue, with now and then the threat of an appeal to higher authority. At last she changed her methods. "Well, I thought I knowed some tol'bl' good chillens, but I sees I done mek a mistake; I sees you is a set er lnow-hit-alls, whar kin gire p ints ter yo' maw an' yo' 'Tncle Hinry an' all de growed-ups, let 'lone a ol' ooman lak me. De times is mo' diff" nt f"um w'at dey wuz. In my day an' gineration de ol' folks had charge er dis worl', but now de roung folks done tucken de reins in han' an' de ol' folks gotter go jes' w'en an' whar dey sesso. Dey jes' lets us live on top de yearf an' dats 'bout all. Things gittin' so topsy-turry hit mek my ol' haid spin roun'. Dar ain' bin nuttin' lak hit sence de day w'en young Mistah Crab think he know mo' dan de One whar done mek him."

The children pricked up their ears at the prospect of 207 


\section{AT TIIE BIG IIOLSE}

a possible story, but it would not to to capitulate too soon, so they went on playing with half of their attention directed to Aunt Nancy, as she stood beguilingly in the doorway.

"Nemmine," she said, "Crab done met wid his comeuppance 'long er his smarty ways, an' I boun' you some chillen gwine do de same ef dey ain' stop dish yer foolishness an' come right in outer de wet. Does y'all think you wuz raise' in de duck-pond an' kin shed de. water same ez ef you had fedders an' walked wid a waddle an' talked wid a quack? I gwine wash my han's er de hull bizness. I done said de las' wo'd, an' y'all kin stay ont yer 'twel you gits water-soaked, lak dem ol' logs down in de swamp, fer all I kyare."

With that she disappeared from the doorway, followed by the children, eager to make peace and to learn the story of the naughty Mr. Crab. Mry shoes and stockings were first in order, and then, her little charges seated with her about the fire, Iunt Nancy leaned comfortably back, ready to satisfy their curiosity.

"Now I 'spec' you chillen s'pose dis yearf alluz bin jes' de way 'tis now," she began, "but w'en you git ol' 'nuff ter read yo' Bible you gwine fin' out dat der wuz a day an' time w'en dar wan't no yearf a-tall an' de Lawd jes" hatter set ter w'k an' mek hit all f'um de bottom er de lowes' sea ter de top er de highes' mountain. W'ats mo', dar wan't no bushes ner trees ner grass ner flow's: Ife hatter mek 'em all, an' atter He git 'em done hit look so fraish an' green an' bloomy dat hit seem lak der orter be sump'n er ru'rr ter walk roun' an' look at hit an' mek 'miration over hit. So den He set ter wu'k on de creeturs. 


\section{WHY CRABS WALK BACKWARD}

"Honers. dat wuz a big job! He jes" had one pattern ter go by, an" all dar wuz to hit wuz body, laigs, haid an' tail, an" rit Ite done mek "em er"y one difi"nt. He gin some un "em fur an" some fedders an" some jes" nuttin' but de naked hide: an" some got long. bushy tails, an" some come mighty nigh gittin no tails "t all. He strotch de laigs fer some an "lef" "em shawt fer urrs, an putt on a li"l mo' claw yer an a li"l les dar, an' took a pinch er dis off rer an" stuck a dab er dat on dar; an" gin dis bu'd mo' wing-fedders an' dat $m$ mo' tail-fedders, an' changed His min' 'bout some er de naked 'uns an' kivered 'em wid scales, an' got out his paint box an' bresh an' tetched 'em up yer an' dar. an' las" dey wuz all done, 'scusin' der haids, an' dey wu er'y las' one diff'nt f'um tu'rr. He sort er rum shawt or goonds w"en IIe git ter Mistah Snake an' Mistah Trum. so dem creeturs got neener fur ner fedders ner laigs ner footses.

"I tol" you dey wuz all done 'scusin' de haids. Well, de Lawd tuck an' putt 'em in a row in front $n$ 'im, an' den He sot ter w"k on de haids. He lef" dat ter de las", 'kase He knowed 'twuz de mos' empawtant, an' 'kase He cu'd tell dat-a-way w"ich sort er haid gwine bes' suit w"ich sort er creetur. He knowed twuz a ticklish job an" dat He mought sp ile de hull bizness ef Te ain' kyarful. Same ez wid de bodies, He jes' had one patterntwo eves, two rears, a nose an" a mouf-yit, same ez wid de bodies, He made "em all diff"nt-nose long er shawt, mouf big er li"l, eves close toge'rr er fur apart, rears long er shawt, sharp toofs er blunt toofs er no toofs a-tall. De older you chillen gits de mo' you gwine ax ro'se'fs how "tis dat er'y man, onman an" chil" you meets has jes' got two eres an' a nose an' a mouf, an' yit you 


\section{AT THE BIG HOUSE}

kin go up de yearf an' down de yearf an' you ain' gwine fin' nair' two faces jes' zackly de same. An' dats de way 'tis wid de creeturs, too. Dey may look de same ter us, but I boun you dey looks jes' ez diff'nt in de face ter one nu'rr ez w'at we-all does ter ow' own kin'. Now dars dat houn' dog er mine, old Bouncer; dat 'ar dog so smart he mought jes' ez well be human; I ain't got nair' fault ter fin' wid him, 'scusin' dat he will trifle wid de ash-cakes w'en my back is turnt; but dat I gotter 'spec' f'um him, 'kase 'tain' poss'bl' ter fill up de kyarkiss ur a houn' dog, 'tain' de nater er de beas' ter feel fill' up. Well, suh, w'en I go up ter de co'te-house on co'te-day an' teck dat dog wid me-w"ich I mos' in gin 'ly does, 'kase I knows he wanter see his own kin' now an' den, same 'z I does mine-he jes' gins one look inter de face ur er'y dog he meets up wid, an' dat minnit he knows ef he done seed him befo". an' ef he has, he stops an' tells him howdy, an' ef hits a strange dog, he knows by dat one look whe rr de dog is a shif less, low-down cur, er whe'rr hits wuf de w"ile ter mek his 'quaintance. Ef 'tain', mebbe he'll gin him a nab, jes' ter let him know his betters is passin', er mebbe he'll go rarin' by widout even turnin" his haid, jes' ter let him see dat he's too low-down fer Bouncer ter notuss, let 'lone bark at.

"Lessee, how fur 'long wuz I wid de tale? Yas, uhhuh, whar de creeturs wuz all made an' done an' stannin' up in a row fer der haids ter be putt on. I tell you dat wuz a mighty ticklish time w'en de Lawd have all de haids pile' up. waitin' fer de owners, 'kase hit moughter bin dat de wrong haid an' body got toge'rr, er dey 


\section{WHY CRABS WALK BACKWARD}

moughter bin j"ined slanchways, er dey moughter bin a po' fit, but den dish yer wuz de Lawd doin' dis, so in cose dar wan't gwine be no slip-up.

" 'Come rer, Lion,' sez de Lawd, sezee, 'an' lemme putt yo' haid on ; hits monst'ous hairy ; don' keep me waitin',

"so Lion he walked right up an' got his haid on in a jiff. an' den shuk his mane an' gin one big roar an' went splungin' off inter de woods.

.. Come yer, Efalent,' sez de Lawd, sezee, an' wid dat Efalent walk up an' git his haid on an' go twis'in' an' curlin' his trunk an raisin' up his voice 'twel de groun' plumb shuk wid de soun'.

'.Come rer, Hoss.' sez de Lawd, sezee, an' wid dat Hoss prance up' an' git his haid on an' go off neighin' an' r'arin' an' kickin' up his heels same 'z de hosses orer gonner in de paster.

" 'Come yer, Cow' sez de Lawd, sezee, an' Mis' Cow santer up an' git her haid an' go off shakin' her hawns an' chewin' her cud an 'mooin' lak she might'ly please' wid hussef.

“'Come yer. Dog,' sez de Lawd, sezee, an’ wid dat Dog he go trottin' up an' git his haid putt on an' go off waggin' his tail an' flappin' his years an' barkin' at er ybidy he meet.

"Jesso 'twuz wid all de creeturs. f"um de Efalent down ter li'l ol' Mis' Ant. an' dey wuz all please' an' sassified. but w'en hit come ter de turn er Mistah Crab, he hilt back. an' he say, 'Naw, suh, 'scuse me, suh, ef I ain' come formu d.'

"Tu'rr creeturs wuz all scannelize' at his imp'ence, an' dey nudge him an' tell him ter go 'long an' quit his foolishness. 


\section{AT THE BIG HOLSE}

"Come ver. Crab," sez de Lawd, sezee, 'an' lemme year w' at "enses you kin mek fer dis 'harishness.'

"Irid dat (rab picked up his body on dem long, spindly laigs er his"n. an' went sidlin' an' teeterin" up in front er de Lawd, fer all de worl' ez ef he wuz walkin' on stilts.

".Please, suh,' sezee, 'you mus' 'scuse me, suh, but I ain' want you ter putt my haid on me: I knows 'tain' gwine feel comf"ble. I wanster putt my own haid on, 'kase hit stan' ter reason dat I mus' know better'n anyone else w'en hit feels jes' right.'

"Ef you"ll b"lieve me. dar he stood, sassy ez a jay-bird, sputin" an" argyfrin "twel de Lawd wuz plumb wo' out wid him an' gin him de haid, right side up, jes' de way twuz ter be putt on. Crab he tucken hit inter his claws an' hysted hit up even wid his body an' clapped hit on. 'Ouch!" sezee, 'dat kain't be right, feels sort er lopsided: an' wid dat he gin hit a li'l hitch ter one side. Mo' wusser dan befo"! 'Shucks!' sezee, 'dat won' do ; feel lak I sort er whopper-jawed,' an' he gin hit nu'rr li'l hitch. He kep' hit up dat-a-way 'twel he had de haid on plumb upside down an' den he wuz sassified, an' widout so much ez 'thanky' de ongrateful creetur putt out fer whar he camed f'um, w'ich 'twuz down by de sea sho'.

"But bless yo" soul, his idees wuz all turnt upside down 'long wid his haid. an' w'at did he done but run backwu'ds stidder forw'ds.

“'Lawsy" sezee. 'seem lak I ain' gittin' over de groun' same ez tu'rr creeturs. W'ats de marter wid me! Look lak ev'ything rumnin' way f'um me stidder gittin' closter to hit. 'Spec' I better go back an' ax 'bout dis.' 


\section{WHY CRABS WALK BACKWARD}

"Tell, suh. he had de insurance ter go backin" hisse"f up in front er de Lawd an' ax Him 'bout de marter. but de Lawd jes' shet spang down on him an' tol' 'im dat sence he done tucken his haid in his own han's he mus' stan' de commonsqinces ef 'twan t putt on right.

"Trer sence den de crabs bin doin' der rumnin' backwu'ds, an' ev'y now an' den you' 11 see 'em stop an' do lak dey sort er stracted by hit an' sline ofl a lill ter one sides an' fiddle roun' wid dem spidery laigs a li’l, an` den go on ere”n. an' folks tells me lat ef crabs gits one'r dem laigs hurted dey kin jes' drap hit off, easy ez wink, an' grow anu'rr jes' ez good ter teck hits place, dat dey kin; an' I kain't see w'y 'tain' fix up so 't folks kin do de same, 'kase 'pears ter me dat folks got jes' 'z much use fer laigs ez w'at crabs is, an' mebbe mo so. But 'tain' no nse ter ax queschins bout de rumnin'-gear er dish yer worl, 'kase dey ain' gwine be arnsed, an 'tain' gwine change de fac' dat crabs kin grow new laigs an' folks kain't. Ef you turn outer yo way a lïl atter one dem crabs he gwine gin you a nip ef he git de chanct, an' he look jes' ez mad, skeedarldin' backwu'ds orer the san', ez ef he harl a spite 'gins' de hull creashun 'kase he hatter walk backwu'ds. An' I ses ter you. dats de way wid dese mulish folks all de worl' over; tecks der own way 'bout er'ything, an' den w'en hit don 'gree wid em, wanster teck der spite out on urr folks. Heap easier ter git mad wid urr folks lan tis wid yo"se'f.

"Now dat's de tale, jes' ez 'twuz tol' me by a gal f' Baltimo', an' I reckon she orter knowed all 'bout hit. 'kase dey tells me Baltimo' is a gre th place fer crabs an' oysters an' all sech truck ez dat wat we don' grow in dis kyounty." 


\section{AT THE BIG HOUSE}

Just then little Kit gave Aunt Nancy an unlucky hit on the elbow from a stick with which he was playing. She seemed more put out than the occasion warranted, but explained it by saying that if your crazy-bone was hit you were sure to be disappointed. Ned, who was a very matter-of-fact young person, inclined to believe only in the things he saw, wanted to know if she really believed in signs like that. As on a previous occasion, she expressed firm faith and added that she believed there was scarcely anything which happened that was not a sign if you only knew how to read it aright.

"Urr folks," she said, "has tuck notuss er dat sidesen me, an' dey done putt 'em inter rhỵmes an' jingles an' sech, so 't folks kin 'member 'em better. Mebbe I kin gin you some un 'em. Lessee, dar's one ses,

\section{'Right han' itch, rub on wood}

An' say, come good, come good, come good;

Lef' han' itch, rub on brass

An' say, come fas', come fas', come fas'.'

"Ef you'll say dem wu'ds an' blow on yo" han' an" den shet hit tight you gwine git a present. sho', sooner er later. Ef yo' years itch, some un talkin' 'bout you an' you mus' say ter yo'se'f,

\section{'Talk' good, good betide you; Talk bad, devil ride you.'}

"Ef yo' eye itch, you gwine see a stranger, an' ef yo" mouf itch you gwine git a kiss f"um a stranger. Sho! dar's mo' signs dan you kin shake a stick at, but you kain't 'spec' me ter think 'em all up ter onct. I done gin you 'nuff fer one time." 


\section{THE ORIGIS OF THE CAT}

The children liked Aunt Nancy's account of the creation so well that they begged for more along the same line. and Aunt Nancy readily obliged them, though her dignity seemed to require that she should begin, as she often did, with a make-believe protest.

"I "clar" you-all dunno w"en ter stop," she said, "but I spose I gotter mek up my min' to hit, fer a po' ol' nigger ooman lak me, w'en she gits wid a set er chillen lak you-all, ain' got no mo' chanct fer havin' her own way dan a chicken-fedder in a harrycane."

she put more wood on the fire, fanned the coals into a blaze with a huge turkey-wing, afterward sweeping the hearth clean with the same implement, administered correction to the insinuating Bouncer, who had followed her up to the "big house" and installed himself, by almost imperceptible degrees, in the warmest place before the fire, and settled herself to begin a new story,-one that concerned the origin of the cat.

"Dese yer cats," she said, "ain' got no call ter sling on airs an' putt up der backs at folks de way dey does. 'kase dey wuz de las' er de creeturs ter be made. long atter all de res', an' dey orter know der place an' 'have derse'fs 'cordin'. Not dem! Dey wants de bes' ur ev'thing wharuver der goes an' ef dey kain't git hit by hook, den dey gits hit l,y crook. Seem ter think dat 


\section{AT TIIE BIG IIOUSE}

meat-platters an' cream-jugs wuz made on puppus fer dem ter nab der livin' f'um, an' de softes" cushoms made fer 'em ter lay on, an' you kain't git dem idees outen der haids.

"Mos" times you kin subjue a dog, but de cat she jes' comes bobbin' up ag“in, springy ez a injy-rubber ball. Ef' you don' b'lieve dat, jes' watch ol' Snip, dat ol' valler torm cat ur Aunt 'Phrony's. She kain't do nuttin' wid him; she done met her match fer meamness in dat cat, an' I reckon dat huccome she keep him. Dat cat ain' 'feard er nuttin' ner nob'dy; he do jes' 'zackly de way he wanter, an' 'Phrony husse'f don' dast ter mek him git down outen her cheer. He done got ter de place whar he 'bout think de cabin b'long to him, an' he jes' lets de urrs stay rom' wid 'im. Hit sut'n'y is a sight ter see 'im wid 'Phrony's ol' dog. Snap. Sometimes he lets Snap lay on de ha`th, an' sometimes he don', an' w'en he gin de w'd ter go, I tell you dat dog gotter git up an' dus' er de fur gwine fly. I done seed Snip git right on Snap's back. whar Snap kin't reach 'im, an' jes' ride him right out de do' an' see 'im clean offen de primises. ' 'mph! W'at 'twix' dat dog an' dat cat an' dat boy Wi'yum, dat ooman sut'n'y have trials an' tribilations! But 'bout de tale, I mus' keep on de track better'n dis, er I ain' gwine git ter de een' in time ter git home an' git my ol' man's dinner.

"Long befo" cats wuz made er thought ur, dar wuz a ooman lived all by husse'f; one dese yer lone wimmin whar dey calls ol' maids. Her cabin wuz 'way off in de woods som'ers, an' she ain' have no near nabers ner no pusson ter look atter her. "Twan't so bad in spring w'en de dogwoods wuz in bloom an' all de flow's bustin' 


\section{THE ORIGIN OF THE CAT}

thu de rearf, ner in summer w'en de sun shine bright thu de wooks an' wo'm up de pine-tags "twel dey 'bleeged ter let out der sweetness, ner in de fall w'en ev"ything good wuz ripe an' she wuz pow ful busy gittin' in her cawn an' taters an' punkins an' her wood fer de col' wedder. But w'on de winter done come an' de pine trees rock 'twel hit soun' lak waves dashin' on de sho' an' de wind howl 'woo-hoo-oo! woo-hoo-oo!' all roun' de house an' down de chimbly, den, I tell you, dat ooman felt mighty low down in her min' an' wish't she d tucken de fus" man whar axed her ter have 'im; an' she'd say ter huscef. 'My lan', my lan'? seem lak de som' er dat wind jes' lonesomes me ter def."

"She had a heap or boddorments, too. De hawks an" de minks an de foxes done stole her chickins outside de house, an' de rats an' de mouses dey gnorred her cawn an' her 'taters inside de house, 'twel she say: 'Humph! seem lak I jes' livin' dese days fer ter raise truck ter feed de varmints wid. 'Pears lak dey done j'ine in cahoots fer de puppus er starrin' me outer dese diggin's. Ain' lef' me 'nuff ter do me 'twel nex' crap, let 'lone seerl-cawn an' seel-'taters.'

"Sidesen dat. she wuz 'feard er de rats an' de mouses, mo' 'special de mouses, an' dey wuz smart 'nuff ter know dat, 'kase de creeturs alluz seem ter know w'en folks is 'feard un 'em. so dey jes' put derse'fs ter w'k ter lead dat noman a darnse. Dey'd set an' watch her f"im der holes an" den scoot out an 'mu right cross her footses an mek her screech an' gadder her skirts up, an' den dey'd go down in der holes ag'in an' laugh an' snicker toge ir 'bout 'dat fool ooman in de room up yonner.' Dey dogged 'er an'tripped 'er up, an' jumped out at 


\section{AT THE BIG IIOTSE}

'er f'um behime things, an' hid in de meal-bag an' run out at 'er w'en she went ter' git de meal, an' dar wuz no een' ter der gwines-on. Las' dey got plumb owdacious, an' at night, atter de ooman done gone ter baid dey'd hol' reg'ler jamborees, eatin' her cawn an' 'taters right spang befo' her face an' eyes, an' scootin' an' scamperin' an' squeakin' 'twel she cu'dden git her natchel slumbers. She'd lay dar quakin' an' shaken' an' trem'lin', 'spectin' ev'y minnit dat de sassy li'l cusses 'ud climb up on de baid.

"One night dey got mo" rambunkshus dan urer befo'. Dey played pussy-wants-a-cornder an' blin'-man's-bluff all over de room, an' hide-an -seck in de sugar-bowl an' bread-box, an' tagged one nu'rr all roun' de aidges er de milk pans. Den dey got tired an' sot down fer a minnit ter ketch der wind an' study w'at debilment dey kin do nex'. All ter onct, de ring-leader, a mighty scampish li'l chap, sot up suddintly on his behime laigs an' fotched a lond squeak, an' sezee: 'Heyo, boys! yer's whar I gwine hare fun wid de ol' gal up yonner in de baid. Does any un you dast me ter do hit?"

"Co'se der gin him a dyare torreckly, an', wid dat. he spit on his han's an' tucken a run an' a jump an' skunt up de bed-pos' clean ter de top, an' den he sung out:

'One fer de money, two fer de show", Th'ee ter mek raidy an' fo' fer ter go ;'

an' wid dat he turnt a han'-spring smack down onter de ooman. Dat'uz too much fer her' she gin a yell lak a wil' Injin an' bounced outer baid right in de midse er de mouses an' skeert 'em inter de holes; but w'en 


\section{THE ORIGIN OF THE CAT}

dey wuz safe inside dey sot dar pokin' der haids out an' twitchin' der whiskers at "er.

"Now ain" dis jes" too much!" she say; "me dat bin feedin' an' housin' dese li"l rapscallions right erlong. Look lak dey monght lemme 'lone w'en sleepin' time come,' an' dar she sot trem 'lin' an' wringin' her han's an' callin' on de Lawd ter he 'p her.

"Twuz a pow"ful col" night. an pres"n'y she yearn a monst ons scrunchin' on de snow, de way you kin sear hit w'en de wedder is col" an' cle'r. De steps come closeter an' closeter, an, hit som' lak de hills an' de mountains had tucken ter derse'fs footses an' wuz comin' to'des her. Las' de steps stop befo' de house an' dar come a knock on de do' dat soun' lak thunder, an' de ooman'so palyze dat she sca'cely lin say 'Come in.' She say: 'Oh me, oh my! w'at gwine happen ter me, now! Ef 'tain' one thing hit's anu'rr. Seem lak dar's no een' ter de troubles er po' lone wimmin!' Wid dat she fell to crrin' ag "in.

"Den de do" flew'd open an" dar stood de Lawd hisse'f come ter he'p de ooman. She wuz so faintyfied dat she cu dden raise huse 'f f"um de flo", but de Lawd ain" fault 'er fer dat, 'kase in co'se He knowed jes' how she wuz feelin'. He ain' say nuttin' 't all, jes' tucken de gre"t fur glore f'm His han' an' thowed hit on de flo" whar de mouses bin, an' lo beholst you! hit 'gun ter wiggle an' squ'm a li'l, an' de ooman's eres 'mence ter bulge w'en she see dat. She rub 'em onct er twict an' say ter husse'f, 'Laws-a-mussy! is my eyes done 'ceive me, er is dat glore a-morin'? Hit sut'n'y wuz, an' w'at's mo', right w'iles she wuz lookin', she seed de thumb turn inter a tail an 'de fingers into fo' laigs an' 


\section{AT THE BIG HOCSE}

de ga'ntlet inter a haid, an' de creetur 'gun ter strotch hitse an wuk hits claws an sniff at de mouse tracks, an' pres'n'y hit made a spring at one'r de li'l skeezicks whar done poked his haid out too fur, so"st he cu'd look at de stranger, an', bless goodness, de creetur ketched dat mouse in a twinklin' an' gin him one sho '-nuff bite an' a good raggin' roun' an' come an' laid 'im down, stone daid, in front er de ooman, jes' ter show her w'at he cu'd do fer her.

"She wuz so might"ly please" dat she tucken de cat"kase dat w'at "twuz-in her arms an" 'menced muchin' hit, huggin' hit an' strokin' hits fur an callin' hit pet names, an' de cat wuz so please', too, 'kase hit seed hit done mek a fren', dat hit jes' squat right down in her lap an' 'mence ter sing an' purr; an' dat de way cats bin doin' urer sence, w'en dẹ feels right good an' fren'ly. Hit mek dat noise so much, dat las' de ooman go dat-a-way, too, w'en she wanter call hit, an' de cat got ter thinkin' dat wuz hits name an 'ome a-runnin' ery time, so de ooman she say she b'liere she name de cat 'Purree.'

"De ooman done fergot dat de Lawd wuz stannin" in de do", an' w'en she turn roun' ter thank Him, de do' wuz shet an' 'twan't no one dar, an' she think mebbe she done dream hit all. But 'twan't no dream. an' dis w'at prove hit; down unter dis day de cats hunches dersef's up w'en dey gits mad, jes' fer all de worl' de way de fingers clinch up to'des de han' w'en folks git mad 'bout sump'n ru'rr, an' down unter dis day de wimmins is still 'feard er de monses. mos' un 'em, an' I 'spec' dat's w'y dey kyare mo' fer cats dan de men-folks does,-mo' 


\section{'THE ORIGIN OF TIHE C.TT}

'special de ol' maids an' lone wimmin in gin'l, dey is plumb sot on "em."

Aunt Nincy was struck by an afterthought and addert: "Ol" 'z dat ol maid wuz, I boum' you dar wuz one thing she tuck good kyare not ter do, an' dat ruz nuver ter tromple on dat cat's tail."

"Why not, Lunt Naner" asked traney.

"IVY, honers," said she, "ain" you nurer year tell dat of you tromple on a cat"s tail you neenter "spec' ter git ma ied dat year? Co'se she ain' gwine git on de cat's tail ef she lin he'p hit." 


\section{THE DRAGON AND THE THLNDER}

One day when the children were down near Aunt 'Phrony's cabin, Ned killed a harmless snake of considerable length with which he proceeded to give himself, boy faslion, a great deal of amusement by swinging it around perilously near to Janey and little Kit, who shrieked and retired to a safe distance, yet, impelled by a fearful fascination, drew near again and again to be routed anew by Ned's uncanny plaything. Haring reduced his victims to the rerge of tears, he promised to keep the dead snake within bounds, and said he was going to take it to Aunt 'Phrony and get her to measure it for him.

Aunt 'Phrony proved to be no great friend to snakes. She brought out a rude yardstick and told Ned to do his own measuring. "I ain" gwine tetch dat thing, 'deed I ain"," she declared. "I got no use fer snakes, nohows. I bin dat-a-way urer sence I kin 'member. Jes' lemme meet up wid Mistah Snake in de paf an' I gin him all de room w'at dey is. I don' stop ter pass de time er day wid him; I jes' open my mouf an' gin a beller, an' den lif' up my dress an' shake de skirts, an' den my footses do lak dey have wings to 'em an' kyar' me outen dat widout me sca'cely puttin' 'em ter de groun'. Shoo-oo! go 'way snakes!'”

"Ho," said Ned, contemptuously, "I don't see any- 


\section{THE DRAGON AND THE THEXDER}

thing about snakes to make people act that way. What makes you so afraid of them !"

"Has I tol" you I'se "feard un em? I dunno'z I is. I jes" do so widout stoppin ter think ef $\mathrm{I}$ is "feard er ef I isn". I don teck time ter git skeered. I dumno w' I ac's lak dat, "thouten mebbe hit's "kase my mammy nseter tell me "bout dat ol sarpint whar temp' Mis" Eve in de grardin", an' lak ez not w'en I come 'cross one hit run thu my min' dat meblbe hit's de $\mathrm{Ol}^{\circ} \mathrm{Boy}$ hissef comin' fer ter temp" me. Sidesen dat. w'en I wuz a li"l gal my daddy useter skeer me tellin' me "bout a big ol snake whar dey calls a dragon."

"That's a dwagon. Aunt "Phrony"" asked Kit.

"Well. honer:" she said. "ez near ez I kin mek hit out, a dragon is one $\mathrm{r}$ dese yer watcher-may-call-ems dat's easier ter name dan "tis ter splain. Anyhows, daddy useter say "twuz a gre"t long snake wid monst'ous eres an' seben big spots on hits body, an' you kain't ketch hit an' you kain't git 'way f'um hit, an', 'lessen you do lak de man in de story done, you kain"t kill hit."

"I wonder what the man did." remarked Janey to no one in particular. Aunt 'Phrony pretended not to hear, while she lit her pipe and puffed away in silence for a few moments. Then she settled herself back in her splint-bottomed chair, slowly blew out a ring of smoke and began the story.

"De man in de tale." she said, "wuz a hunter whar bin out atter bar. He wuz gwine "long thu de big mountains w'en he rearn a turr"ble noise, so loud dat hit soun' lak de mountains done tore de hills up an' wuz chunkin' 'em at one nu'rr. De man 'gin ter git skeered an' trimmle lak a aspum, but yit he git nearer 


\section{AT THE BIG HOLSE}

an' nearer, an' de noise git louder an' louder, an' las' he seed 'twuz a IPragon fightin' wid de Thunder. De two wuz so tankle up wid one nu'rr dat de man kain't see de Dragon's haid, wich 'twuz well fer him he kain't. But Dragon see him an' call out ter him fer he'p. He say: 'Please, Mistah Man, ter come yer an' he'p me gin Thunder sump n dat'll keep him quict fer one w'ile. Gwine roun' rer, he is, strakin' de trees an' killin' de béas'eses an' doin' all sawts er mischief! Fus' you know, he gwine strak you daid some dese days, "fo' you kin tell w'at kilt rou.'

"Den de Thunder he call out ter de man in a gre"t big roice dat mek de yearf trimmle, an' he say, he do: 'Boom! Boom!! Brr-oom !:! Lissen at me, Mistah Man! You bes' he p me outen dis scrape. Ef you hep Dragon hit gwine be wusser fer you, kase ef you git one good look at 'im, right in de eye, you boun' ter die; no he p fer hit. Folks whar sees a dragon mought ez well turn up der toes right den an' dar.'

"Man he study an' he study. He say ter hisse'f, sezee: 'Well, dish yer a pooty kittle er fish! Ef I he'p de Thunder, lak ez not he gwine fergit bout hit 'an strak me daid some time er ru'rr. An' ef I he'p Dragon I hatter look him in de face atterwu'ds, an' dat 'nd be de een' ur me. 'Pears lak w'ichurer way I jump I mought git f'um de fryin'-pan inter de fire.'

"Las' he made up his min' dat he'd have a better chanct wid de Thunder. By dat time Dragon done got de Thunder all tied up. jes' wropped hisse'f roun' an’ roun' him. Thunder gittin' kind er choky an' shawt er bref. but he mek out ter call ter de man, in a rumbly voice dat soun' lak 'twuz dyin' 'way 'mongs' de moun- 


\section{THE DRAGON AND THE TIINDER}

tains: 'Ob. Mistah Man! Please, Mistah Man, git me onloose foum dis ongawdly creetur. Shoot him in de sebent -pot, sul, dat'll fotch him; shoot him in de sobent spot:"

"so de hunter he up an shoot de Dragon spang in de sebent spot. an' dat onlonse de Thunder. Dragon bin hol"in on so hard dat w'en he let go. de Thunder riz cte rep inter de clouds an den fell down ag: in wid a crish an a smash an' a bang right onter de Dragon an' tored him inter sassidge-meat.

"Den de Thunder he say ter de hunter: 'Rr-um! Dr-um! Bum! Mistah Man, dish yer a mighty good day's w'k you done fer me an' fer de people er dish rer kyountry, 'kase dat Dragon bin gwine 'roun' mekin' a noosance ur hisece. Now. suh, "kase you done bin my fren dis day. I gwine to sumpin fer you. I gin you my wid dat you kin come an you kin go thu de storms an I ain "wrine tetch nair" hra'r er ro haid. Sidesen dat, I gwine gin you de power ter strak de trees an' kill em. same z I kin. W'at's mo', w'en you goes inter battle an yo inimies seem lak dey gwine git de bes' ur you, jes you call pun my name an you gwine orercome 'em ery time. Io man kin stan up 'gins' de Thunder. Son, you year me: de wu'd bin spoken.'

“Den de hunter say ter hissef, sezee: 'Tm-umph! I reckon I bes mose 'long outen dis. 'kase I kaint tell how sonn ol man Thunder mought change his min'. Te foelin' right good an' sore jes' now f'um de quiles er dat Dragon. but how does I know w'at he gwine do w'en he wit orer dat? I bes' quit 'im w'ile he's feelin' a li'l inncer.' So he mek his manners an' kotch up de b'ar-meat an' putt out right smatually foum dat. an' I 


\section{AT TIE BIG HOT'SE}

ain so sho" but w'at he tuck an" bruk inter a run soon ez he got roun' a bend in de mountains."

The story finished, Ned returned to his snake and was about to cut it up to see if the separate pieces would wriggle, as he had heard that they would do, when Aunt 'Phrony warned him to desist.

"Souls an' bodies, chil"." she exclaimed, "you better stop cuttin' dat snake ter pieces. Ef you wuz ter do dat, an' lef' de pieces rer, ev'y snake dat wuz a fren' er dat un w'ile 'twuz livin' 'ud come quilin' 'long up yer ter git de pieces an' try an' putt 'em toge rr ag in. I ain’ want my back yard ter git full er dem squ'mmy things, so I jes' ax you ag̃in ter lef' dat snake be, 'kase I know w'at I'se talkin' 'bout; hit's de sho'-nuff trufe." 


\section{THE TLANIIA}

"Ir mamma told me," said Ned at the close of the dragon story, "that there were no such things as dragons; people just marle it up about them."

"Look-a-yer, chil"," said Aunt 'Phrony, "I ain' wanter raise no "spute wid yo' maw, but she done l'arr w'at she know f"um books, an' I done l'arn w'at I know outside er books. Dese yer writin' men kain't putt nuttin' inter books but w'at dey fus' git f'um de outside, an' dey ain' putt de hull 'varsil worl' inter books yit, not by a long ways. Io' maw got her book l'arnin', I got my eye-an'-ear l'arnin', an' I knows dis: dar bin all sorts er creeturs an' all sorts er doin's in de ol' days, an' mebbe ef you s'arch up an' down de yearf an' inter all de deep holes an' fur-off cornders, you mought fin' some un 'em yit. Sho! my paw seed wid his own eyes de re'y nes' whar one un 'em useter live; de Tlaniwa, dey call hit.

"De Tlaniwa," she explained, "wuz a gre't big bu'd; bigger, ras, ten times bigger'n any bu'd whar live on de yearf dese days. I'en I wuz a li'l young'un my paw useter tell me, w'en I wuz norty, dat de Tlaniwa gwine git me ef I ain' 'have myse'f. Hits nes' wuz half-way up de face ur a clift on de Chilhowee mountain, wid de Tennessee river roarin' right b'low, so dat 'twuz onposs'bl' ter climb up ter hit, an' de on'ies' way ter 


\section{AT THE BIG HOT'SE}

reach hit wuz ter go roun' an' climb de mountain f'um de back an let ro"seff down de face er de clift. De Thaniwa an his mate felt mighty safe in de nes', an' dey live on dar, 'ear in an' 'ear out, raisin' der chillen an' flyin' all roun' de naberhoods gralbin' up sheep an' lambs ter feed der young'ms, an' nabbin' up young chillen, too, w'en dey cu'd git 'em, fer dey thought chillen mek better eatin' dan anything else. Mr, my ! but dem wuz pow'ful bu'ds! W'en dey spread der wings ter fly hit hide de sun an' ev'ything grow dark, an' w'en dey swoop thu de air de wind rush by so swif' hit seem lak a storm wuz comin'. Folks try ter kill 'em, but dey alluz git away, an' dey scream an' holler an' claw an beat de air wid der wings 'twel er'y pusson glad ter leave 'em 'lone, fer dey wuz so big dat dey cu'd kill even de growed-up folks widout half tryin'.

"Dar wuz a ooman in dat kyountry done have two ur her chillen stole by de Tlaniwa, an' at fus' she go roun mo 'nin' an' groanin' an' kyar'yin' on, but atter w'ile she say to husse'f, 'I givine show dese men roun' yer wat a ooman kin do w'en she try. I gwine redd de kyountry er dem misable bu'ds of I git kilt doin' hit. Dar ain' gwine be no mo' po', innercent li'l chillen chawed up ter mek vittles fer dem owdacions bu'ds.

"She tuck an' tuck a string an' tied sticks all erlong hit an' made a swingin' ladder. Den she go rom' tor de back er de mozntain whar "tain' so steep an' she climb an' she climb 'twel she reach de top. She look orer de aidge er de clift an' seed dat de ol' bu'ds wuz off de nes', gone 'way som'ers ter git vittles fer de young'uns, so she fasten her ladder to a tree near de 


\section{THE TLANIII}

aidge, an' den she climb down, down, down, swingin' 'way out orer de deep, dark river, twel she reach de nes'. She kill de young bu'ds an' thow 'em in de river, an fals" $\mathrm{e}$, she thow "em down a monstous big snake riz un f"um de water an" swallowed "em whole.

"Den de ooman she go an" hide in a safe place an" watch wit te ol buils givine do. Pres'n'y one uv 'em come back wid a young chil' in her claws, an' w'en she sed her li'l uns wuz gone she mek a turr'bl' fuss an' g'long off ter fin' her mate an' tell him de bad news. He come back wid her, an" de two went succhin' an' swoopin' an' screamin' roun' de nes'. 'Th-huh!' sez de ooman to huscef $w^{\circ}$ en she rearn de bu ds mo "nin' an' crrin': "uh-huh! reckon rou-all knows by dis time how hit fcels ter have yo chillen stole an' et up inter de bargum. Tou kin succle an sweep an' swoop, an' you kin scream an' holler an beat yo' wings, but you kain't git back yo" chillen any mon $\mathrm{I}$ kin git back mine whar you done stole an' et, an" she went on dat-away fer a long time, shakin' her fis' an' moufin' at de Tlaniwa.

"De snake he r'ared his haid up outen de water ter see wat mekin" all de fuss. Dar whar Mistah snake mek a mistake, 'kase de bu'ds seed 'im an' made up der min's dat he wuz de one whar kilt der li"l 'uns. "Yis'able, 'ceitful, sneakin', slidin' wretch.' says de Tlaniwa, sezee, "Yer me hin feedin' you wid de bones an' de learin's all dis time. an' yer you done pay me by goin' ter wu'k an' swollerin' my chillen. Gwine teck hit outer yo' hide an taller! Gwine mek minch-meat outen you 'fo' you kin whisp dat ugly tail er yo'n.' 


\section{AT THE BIG HOTSE}

"Wid dat ol" Snake he 'gun ter slip an' slide thu de water. but he cu'dden he "p r'arin' his haid up an' stickin out his ol" fork-ed tongue at de Tlaniwa. Dar whar he wuz mistooken ag̈in. fer de Tlaniwa swoop down lak a streak er lightnin' an' snatch him by de neck an' kyar' him high up in de air, mos' ter de clouds. Ol' Snake he wiggle, he twis', he turn, he squ'm! but he cu'dden git 'way. 'No use, my squ'mmin' fren', sez de Tlaniwa, sezee, 'bes' say yo' pra'rs, kase yer whar I gwine show you w'at a wufless w'um er de worl' you is.' ITid dat, his mate she come an' tucken her beak an' cut pieces offen de Snake w'iles he wuz hangin' f"um tu'rr bu'd's mouf, an' let 'em drap onter de rocks below. Dey went swishin' thu de air an' fell ker-smack onter de stone, an' dey fell so fur an'so hard dat dey done mek deep holes in de rock. "IT'at ${ }^{\circ}$ mo", de pieces turnt ter stone atter w'ile, an' daddy say he done seed de holes, an' done chipped pieces er de Snake outen de rock. An' ef you wuz ter go right now ter ol' Chilhowee. I boun' you'd see de same thing in de rocks at de foot er de mountain whar de river 'rm deep an' strong.

"De bu'ds dey lef" de nes', 'kase dey see 'twan't no fitten place ter raise der young uns, an" dey wan't nurer seed no mo' in dat kñountry. De ooman go home an' tell 'm w'at she done an' de people wuz mighty glad. She tell her chillen an' her chillen's chillen 'bout dem monst'ous bu'ds. so't de people ain nurer fergot 'em, an daddy say all de Injun wimmins useter skeer der chillen w'en dey wuz norty, tellin' 'em de Tlaniwa gwine ketch 'em. Pity dar ain' no tlaniwas in dese days ter skeer chillen whar goes roun' teasin' de dogs an' worryin' de cats whar ain' done 'em no harm." Here 230 


\section{THE TLANIWA}

the old woman looked a sly reproof at Ned, who had the day before been unfeeling and disrespectful to the two cherished companions of her old age: snip, the cat, and Snap, the dog. 


\section{THE 'POSSUM AND THE GRCB-ITORII}

Janey was a reritable little woman in tact and shrewdnes. and when she heard Ned accused of incivilities to Snip and snap, she hastened to turn attention from him by saring the first thing that came into her head. "oh. Aunt "Phrony." she cried. "you haven't told us anything about the Possum for a long time. Don't you know any more stories about him ?"

If 'Phrony saw throngh the simple derice by which Janey meant to kill two birds with one stone, she made no sign, but obligingly racked her brains for more news of the Posum's doings. At length she was able to remember a stor"y about "Mis" "Possum" and "Mistah Grub-wm."

"Dey wuz bofe un 'em mighty fat folks, an' lak lots er fat folks, dey wuz right lazy an' nuver go nowhars dey ain' hatter go, jes' mosey roun' a li'l ter git der vittles an stay still at home de res' er de time, w'ile tu 'rr creeturs all time traipsin' an' trollopin' up an' down de kyountry ter see w'at dey kin see an' year w'at dey kin year an' do wat dey kin do. Mis" 'Possum she stay in a holler tree an snooge de time away, an "Mistah Grub-wim he snuggle down jes' beneaf de top er de grom' an' dream 'bout de time he gwine turn inter a beetle an' live on top de groun'."

"Do grubs turn into beetles?" asked Ned, rather incredulously. 


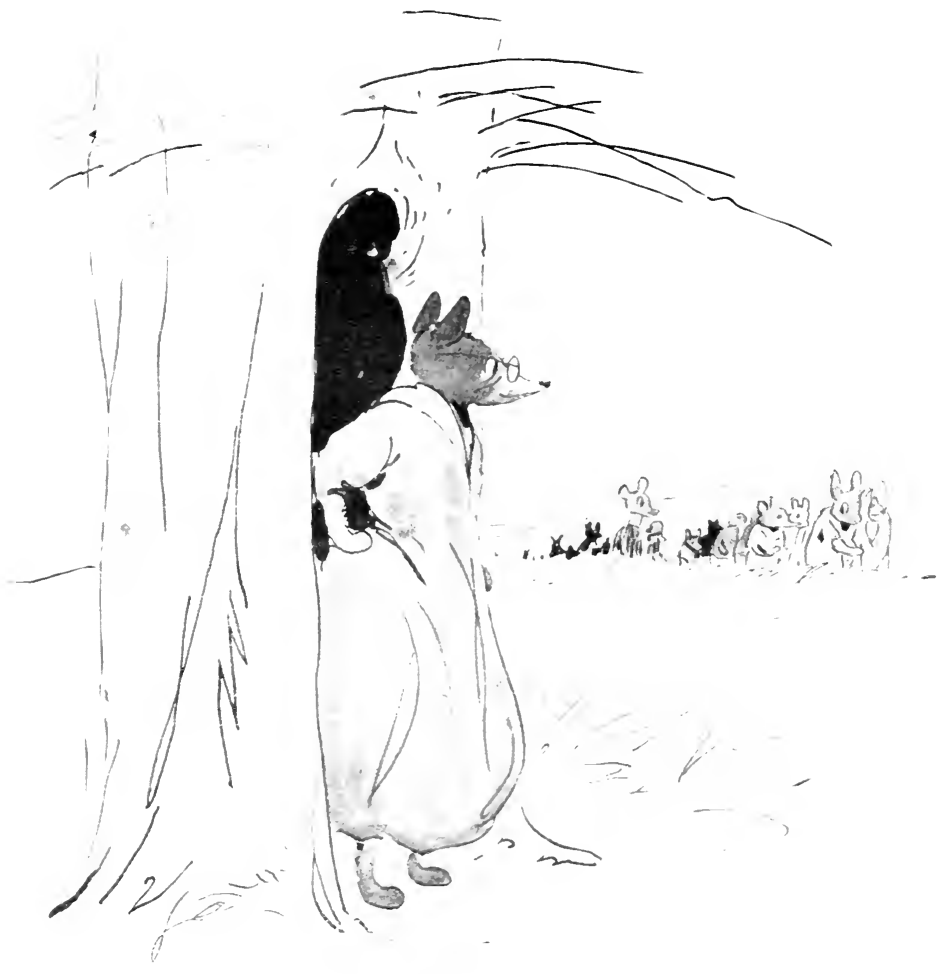

MI' 'PUN-U - HE STAX' IN HER IU 



\section{THE 'POSSLYI AND THE GRCB-ITORMI}

"Yas, suh. dat dey does! dish yer one w'at I tell bout, he lays low un'need de yearf "twel May er June an" den comes out an turns inter one $\mathrm{r}$ dese per posty green an brown beetles whar dey calls 'June beetles: de kin' chillen ketches an sets ter junin"."

Of course the little folks wanted to know what "junin" was.

"Laws-a-mussy." said 'Phrony, "I thought all chillen knowed how ter june a bug. W"y, rou jes" tecks de beetle an' ties a string to "im an' hol's on ter one een" er de string an lets him fly ter de urr een" er de string, an' den he hums an' buzzes an fusses an' cusses, an' dat's wat dey calls "junin?'

"Now. den. lemme start ag"in. I done tol' you dat Mistah Grub-w'm an" Mis" Possum wuz bofe un em fat an" lazy an" lak ter stay home. But dey mighty fond er knowin' wat gwine on in de worl'. an w'en turr creeturs go ter de kyouncil ter "tend ter de fairs er de kyountry, dese yer stay-at-homes ain' gin 'em no peace, w'en dey git back, pesterin' 'em wid queschins 'bout wat went on at de kyouncil. One time some er de creeturs wuz gwine by f'um de meetin', an' Mis' 'Possm she stan' in her do" an' hail 'em an' ax 'em fer ter come in an set a "ile.

"Dey ses, "'scuse we-all. ma'am. we 'bleeged for ter be gittin" on," an' dey ses ter one nu 'rr behime der han': 'Land! land! le's mek has'e an' git outer dis er dat ooman'll nab us an' nail us ter de cheers all night.'

"Mis' Possum she say. sez she: 'Gemmen. I ain' gwine teck no fer a arnser. You-all mus' be plumb frazzle out wid yo" walk. You mus' come in an teck off yo' shoes an' res' yo' huf. an' den lemme gin you a 


\section{AT THE BIG HOTSE}

plate er 'simmons an' a glass er buttermilk. Now, you year me, I ain gwine let you off.'

"De vittles fetched 'em, lak hit alluz does wid de men-folks. an' in dey camed. Mis' 'Possum she ax 'em queschins 'bout who wuz dar an' w'at dey wo" an' wat dey have ter eat, an' wat laws bin pass, ontwel dey wuz fair' wil'. Dey git so wo' out an' aggervex dat w'en she go outer de room ter fetch de 'simmons an' de buttermilk, dey git ter collogin' toge'rr an' fix up a li'l joke on her. W'en she come back she putt down de plate an' de glasses, an' putt her han's on her hips an' stood watchin' 'em eat, talkin' at 'em all de time.

"Dellaws!' she say, 'y'all ain' gin me no news wuf talkin' 'bout. Is dat all you kin 'member er de doin's?'

"Den one un 'em he wink at de res' an' he say, he do, 'Law, bless yo' soul, Mis' 'Possum, I nigh mos' fergit ter tell you de kyouncil done pass a law dat all de anermuels mus' perride derse'fs wid a pouch so s't dey kin kyar' der chillen roun' wid 'em.'

"'Fer de lan's sake!' sez she, 'how I gwine git me any pouch ter kyar' roun' wid me?'

" 'Nemmine,' dey sez; 'we bring you de hide; all you gotter do be ter mek de pouch.'

"So dat w'at dey do. an' Mis' 'Possum she strukken wid de notion dat she save hussef de trouble er kyar'yin' de bag on 'er arm ef she jes' sew hit to er body instid, so, sho'z yo' bawn, she jes' sew hit on her own hide, right in front, an' dar whar she bin kyar'yin' her chillen urer sence. Tu'rr creeturs raise a big hoot w'en dey see 'er gwine roun' lak dat, de onies' one 'mongs' 'em all whar kyar' der young 'uns dat-a-way.

"Mis' 'Possum ain' kyare. She say, 'Gemmen, de 


\section{THE 'POSSLII AND THE GRLB-ITORM}

joke's on me dis time. But sho! 'tain' bodder me. You done me a mighty good turn; dis heap better dan gwine roun' totin' my chillen wid my mouf, de way some er you hatter do w'en you wanster tote yo'n.' An' so ter dis day she teck her chillen ev'ywhar she go, even w'en she go out ter rob a hen-roos'. Sometimes she lays down on de groun' in de sun, an' dey walks onter de pouch an' climbs all orer her an' squats down an' hangs on by curlin' de een's er der long tails roun' her hya'r. I done seed a dozen ur 'em at onct, jes' settin' all crer der mammy an' hangin' on by der tails.

"Atter de creeturs lef" Mis' Possum dey g'long li"l ways an" met up wid Mistah Grub-w'm, an' he turnt to an' 'gun ter pussecoot 'em lak she bin doin'. Dey got jes' natchelly wo" out wid "im, so dey went off a li'l piece ter confabulate toge'rr. 'Now ain' dis jes' too much!" dey ses. "Why n't dis man go ter de kyouncil an' use his own eyes an' years? Mis'able lazybones! So fat he kain't 'ambulate, broad 'z he is long.'

"One un 'em say ter tu'rrs, 'Jes' lef" dis man ter me; I gwine show him huccome!" Den he go back an' he say ter Mistah Grub-w'm, "Mistah Grub, we'all done fergit ter tell you de kyouncil done pass a law dat all anermuels mus' crawl on der backs. Dey say we bin gwine roun' wid ow' eyes on de groun' long 'nuff, an' now we mus' turn over an' look up in de worl',

" 'Lmph!" sez de Grub-wu'm, sezee. 'dat suit me to a gnat's bristle, 'kase I don' reckon folks gwine 'spec' me ter git roun' so fas' ef I hatter trabel on my back. I kin teck hit easy den, sho' 'nuff. Stan' outer de way, you-all, ver goes!' Wid dat he roll over on his back an' try ter crawl. Mighty hard wu'k. He wrassel an' he 


\section{AT TIIE BIG HOLSE}

tussel an' he tug, but he ain' mek no headways. 'Whew! dis mek me tired. sho' 'nuff. I gwine turn me over an' to de ol way' sezee; 'mighty easy fer folks ter set up in de high seats an' gin der orders. I wish all dem dat gin orders hatter try 'em on derse'fs befo' dey kin pass inter laws.'

"He try ter turn back on his stummick, but, bless yo' soul. dar wan't no turn to him. He huff an' he puff, but 'twan't no use. De creeturs dey stood by an' snickered an' aiged him on an' let on ter try an' he'p him. Las' dey cu'dden stan' hit no longer, an' dey bus' out laughin 'an' sez, 'Heyo, Wistah Grub, we done got you fix' dis time. Reckon you won ‘t go pesterin' we-all no mo' wid yo' queschins 'bout de kyouncil. You hatter trabel all de way dar on yo' back atter dis ef you wanter know w'at gwine on. You kain't 'pend on we-all no mo'. So-long, ol' man.'

"Sence den ol' Grub-wu'm bin doin' all his walkin' on his back, an' he bin at hit so long now dat he done got right spry. Sometimes atter a hairy rain he come up ter de top er de groun', an' you kin see him jes' mo'n scootin' roun' on his back.

"Now dat's all de tales you gwine git outen me dis day. I is plumb wo' out, same 'z de creeturs wuz wo' out, wid queschins," and the old woman folded her hands and closed her eyes and pretended to be fast asleep, seeing which her little auditors stole softly away on tiptoe for fear of waking her. 


\section{IIR. BEAR AND MR. TERRAPIN GO COLRTING}

"Yas." said Aunt Nancy, "one time Mistah Bar an" Mistal Tarr"pin wuz bofe co "tin' de same gal. Dey kep' hit up a long time, waitin' on her an' flyin roun her, but neener ur em seem ter git de inside track. Hit. sut'n'y wuz a sight ter see "em gwine on befo" her. cuttin' up shines an' talkin' far' an' trin' w"ich kin 'trac' de mos" 'tention f'um her. B'ar wuz a sulky sort er feller, an' hit look plumb reedikelons ter see him trrin' ter rm on an' laugh an mek hisedf 'greeable. Tarrpin he mighty slow 'bout his talkin'. well ez his walkin'. an' hit wuz 'nuff ter mek a tiger titter ter see him settin' up tryin' ter slip a wo'd in aidgeways now an den. Der"d git dar on de same night. an' try ter see w'ich kin set tu'rr one out, an' neener one ur 'em 'ud leave; so "long 'bout ten de gal's mammy 'ud stir roun' in de nex' room an' drap her shoes dotrn hard on de flo'. an' de gal 'nd be tucken wid a fit er de gaps, an' den dey knower' hit wuz time ter git up an' moser.

"De gal"s folks useter run her right smart 'bout her beaux an' de way dey run atter her an' come roun' nights an' stuck one nu'rr out. an' her mammy 'ud jes' shake all orer wid langhin' an' say. 'Gracione ter goodness. gal! how long you gwine let dem fellers come sasshayin' roun' you? Whyn't you mek up yn min' one way er tu'rr? I kind er tired seein' 'em toge'rr; dey 


\section{AT TIIE BIG IIOUSE}

looks so cur'ous 'long side er one nu'rr. You sut'n'y got de long an' shawt ur hit wid yo' beaux.'

"Gal git kind er tired ur hit, too, but she ain' seem ter be able ter mek up her min'. Las' she say to 'em, one night w'en 'twuz mos' time fer 'em ter leave an' dey bofe wuz pesterin' her ter 'cide betwix' 'em, 'Gemmen, you is bofe so 'greeable dat 'deed I kain't mek up my min' w"ich ter teck an" w"ich ter leave; 'deed I kain't. So dis w'at I gwine do. I'all kin start f'um de cross-roads nex' Friday mawnin' at de same time, an' w'ichurer one gits yer by twelve oclock, dat de one I maly. But I have you bofe ter know dat you mus'n' git yer neener befo' ner atter' you mus' be right on de stroke er twelve er you kain't be de winner.'

"Dey bofe 'greed ter dis an' went on home.

"W'en Tarr'pin wake Friday mawnin' he gin hisse'f a stretch an' open his eyes an' see 'twuz jes' gittin' daylight, an' he say ter hisse'f, 'Shucks! better not be layin' rer no longer: bes' git up an' dus' ef I 'spec' ter git dar ahaid er Mistah B'ar. He ain' no swif' trabeller, dat's a fac', but he's a mighty soon man 'long side er me, so I bes' slick up a li'l an' g'long.'

"So he stop long 'nuff ter gin his shell a good rubbin' down 'twel hit shined lak glass, an' den he sot out fer de gal's house.

“Jes' atter sun-up ol' B'ar wake up an' roll over an' stretch an' rub his eyes, an' sezee, fetchin' a big yawn, 'Dog-my-cats! ef I don' b'lieve dish yer's de day fer de race 'twix' me an' ol' Tarry-long Tarr'pin. Umph! wish't I had time fer nu'rr li'l snooze. Ol' Tarr'pin so slow I b'lieve I gwine turn over an' try a li'l nap on tu'rr side.' Jes' den he ketched sight uv a track in de road 


\section{IIR. BEAR AND MIR. TERRAPIN GO COLRTING}

dat go pas' his house an' he got up ter look at hit. Fus" hit seem lak some un bin draggin" a plank "long thu de dus, but w'en he zamine hit he see de lill foot-tracks on bofe sides an' den he knowed "twuz whar Tarr"pin bin draggin' his un need shell thu de dus:. "Nho!" sezee, 'I better spunk up an' git outer dis: no tellin' how early Tarrpin started: he mought git dar fus" ef I ain' look out.'

"So he gin his fur a good rakin" over an" putt on all his bes' fixin's an' set out fer de gal's house.

"Fo" he got ve'y fur he met up wid Tarrinin an" sezee, 'Heyo, my fren', w'at mek you start so carly? 'Tain' do you no good ter git dar befo' de time. Gal say you mus' 'rive on de stroke er twelre. neener befo' ner atter.'

"Yas, suh: ras, suh. I knows dat, sez Tarrpin, sezee, 'but you mus' member w'at a slow trabeller I is. A big man lak you, wat teck gre't long steps, boun' ter beat me ef I ain stir my stumps an 'start early.'

"So dat's how de lan' lays, is hit?' sez B'ar, sezee. 'Well. ol' man, keep on ef you wanter. but I'se 'feerd you hare all dis trabblement fer nuttin. You mought ez well set down in de shade an "jy yo seet, kase I boun" ter git dar fus': you ain' got de ghos' ur a chanct, an' de gal knowed dat w'en she talk 'bout de race. "Twuz jes' her way ur gettin' shed er you widout hurtin 'yo' feelin's.'

“' 'Tebbe so, mebbe so' sez Tarr'pin, sezee. 'but ef you don' min'. suh, I jes' 'bout think I gwine keep on, now I got dis fur.'

"Dey tol' one nu'rr 'so-long.' an' B'ar he go on an' soon leare Mistah Tarr'pin a fur ways behime. He see de sun ain' ve'y high yit, an' he wuz right wo'm an' tired, 


\section{AT TIIE BIG HOUSE}

so he say he b'lieve he lay down in de shade an' res' a w'ile. So dat w'at he do, an' pres'n'y he go fas' asleep, an 'he slep" an' he slep' an' he snore an' he snore, an' de flies come an' lit on his nose an' tickled him, but he jes' breshed 'em off wid his behime foot an' went on sleepin".

"Atter w"ile 'long come Mistah Tarr'pin draggin' hisse'f thu de dus' an' de hot sun. He see Mistah B'ar layin' off unner a tree, an' he strotch his neck up outer de shell, an' drord off de paf an' looked at him good. 'Th-huh!' sezee, 'fin' asleep, I see. Well, snooge on, Mistah B'ar; dat suits me 'zackly, an' I have you ter know, suh, lat hit's a ol' sarin" an' a true sayin', "De long pole reaches de 'simmon, but de smart dog gits hit."

"Irid dat Tarry-long he went amblin' on an' lef' Mistah B'ar sleepin' lak a log an' snorin' lak a saw-mill.

"Presn'y B'ar waked up an' seed Mistah Tarrpin's track ag'in an' knowed he'd bin by. 'Gee whizz!' sezee, 'dat mis'able li'l cuss done had de insurance ter pass me? 1 mus' git up an' git, er no tellin' w'at happen.' Wid dat he shuk hisse'f an got up an' went 'long right fas' 'twel he ketched sight er 'Tarr'pin ag'in. Den he slacked up a li'l an' putt on lots er style, swaggerin' roun' over de road an' singin' one'r dem ol' toas'es ter de ladiz:

'Success to de red-bu'd,

An' lukise ter de wren;

God bless all de pooty ladiz,

An' not so many men.'

"He let on lak he ain' see Tarr'pin 'twel he git right 240 
up "long side ur "im. an" he mek out dat he wuz je:" bout ter tromp on him by mistake. He dror back his font an say. Oh. "ente me, is dat you: I did n' know you wuz dar. Well. how you come on. anyhow? slow wu'k. ain" hit. suh?" You mought ez well give up an" go back. I tell you dat. "kate dar"s no queschin who gwine git de gal: the knowed dat fum de fus:. I dumno $w^{\circ} \mathrm{y}$ she set me an "rou at dish rer fool racin', but dar"s no use trrin" ter study out de ways ur a gal: I done gin dat up long ago. You gotter let her have her sesso."

"Tarrpin he say, sezee. "Well. Mistah B'ar, rou pussue atter yo" way an" I"ll pursue atter mine. an" mebbe you 'll see me at de een' er de line rit. I done come dis fur "long: seem lak hit be too bad ter gin up now. I'm a right slow man, to be sho". but I tell you, suh, I'm a sticker."

"B"ar g"long an" git way ahaid er Wistah Tarr"pin ag in. He wall his ere up at de sun pres'n'y an' he see dat "twan't twelre rit an' dat de gal"s house wan't so fur awar. so he mek up his min' ter teck nu'rr li"l res'. shucks!" sezee ter hise"f. "Tat lil ol" slow-coach won't be 'long rer fer one w'ile. an' I mus' be hrarful not ter git ter de place befo" twelve. so yer whar I gwine res' me ag"in. Wid dat he plumped hisse of down unneed a tree. He fell fas" asleep befo" long an" he slep" an" he slep" "twel mo:" twelre o clock.

"Atter w"ile yer come li"l Tarr"pin. wo" mos" ter a frazzle, but jes" paddlin" on down de paf" widout eren stoppin' ter teck bref. He see ol' B'ar larin' dar in de shade an' he strotch his neck out ağin fer a gond lnok. an' sezee unner his bref', 'Sn ho, my furry fren'! fas' asleep ag’in. I sees. Dar's whar you mek de gre't mistek er yo' $2+1$ 


\section{AT THE BIG HOLSE}

life. 'Tain' safe, suh, ter 'spise any man, nemmine how po' an' no-kyount he seem ter be; mebbe he gin you a sprise yit one'r dese days. I sez onct an' I sez ag’in, "De long pole reaches de 'simmon, but de smart dog git hit." Wid dat he crope up nearer an' nearer ter Mistah B'ar an' looked him good in de face an' seed he wuz fas' asleep yit. Den he wrikked on down ter one'r Bar's behime footses, an' squat down dar an' tuck de een' ur a tuf" er long fur in his mouf an' sot dar waitin'.

"Pres'n'y B'ar he waked up an' walled his eye up at de sun an' seed 'twuz mos' twelve o'clock. 'Gre't gum!' sezee, 'has dat Tarr'pin pass me by ag'in?' He look ahaid fer Tarrpin's track. None dar, so he feel safe. 'I mus' be humpin' myse'f ef I wanter git dar on de stroke er twelve.' sezee.

"Wid dat he got up an" shuk de dus' outer his fur, but he ain' notuss 'Tarr'pin, 'kase de fur so thick he kain't see him. Tarr'pin wuz hangin' on fer dear life; he wan't gwine be shuk off, not him. Sezee ter hissef, 'Aw naw, suh, you hatter shake hard 'nuff ter onloose de fur f'um yo' hide befo' you kin shake me off ; my mouf built fer hol'in' on. Mebbe I kain't wu'k my jaws ez fas' ez some folks, but w'en hit come ter clinchin' 'em, I'm right dar.'

"B'ar go crunchin' an' serunchin' down de road, mekin' gre't has"e ter git ter de gal's on de stroke er twelve, an' all de time yer wuz Tarr'pin jes' gwine swingin' on down de road wid him, mouf clinched tight in de fur, an' ol' B'ar ain' have de sign ur a notion dat he wuz totin' Tarr'pin to'des de gal's house.

"De gal an' her mammy wuz settin' on de po'ch lookin' out fer de gemmen. Jes' befo' de stroke er 


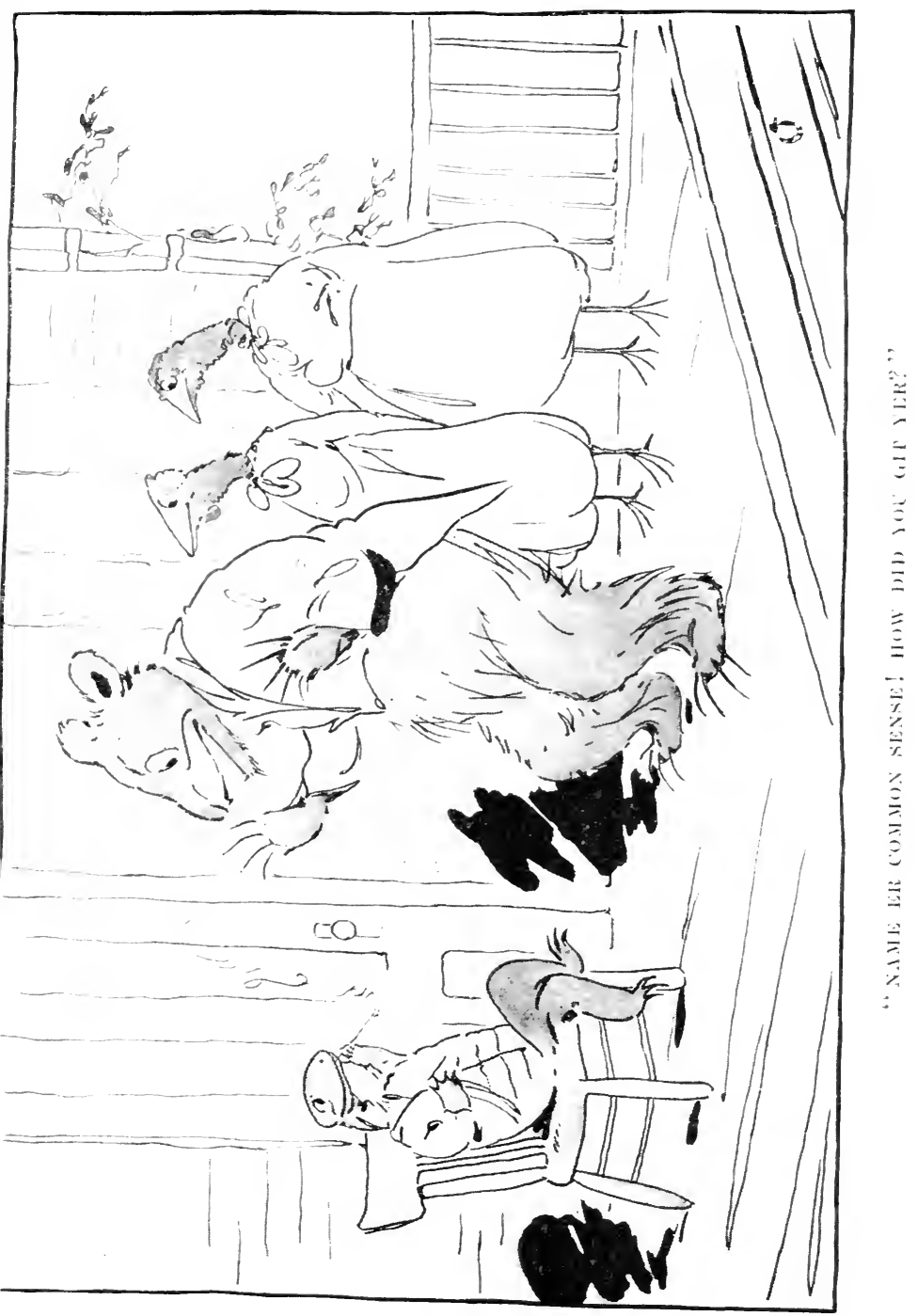



twelve de gal riz up an' shaded her eye wid her han' an' looked down de road. She see Mistah B'ar mekin' gre't hase, an' she sing out, 'Oh, maw, yer come Mistah Bar! Hit look lak he gwine win, "kase I ain' see no sign er Mistah Tarr'pin.'

"B'ar he seed her an' he 'gun ter bow an'scrape an' teck off his hat, an' de gal she 'mence ter snicker an' giggle an' mek her mamers. W'en he got ter de po'ch he wuz clean outer bref, so't all he cu'd say fer a mimnit wuz 'Howdy, ma'am, howdy, gal!' Gal she say. 'Howdy, Mistah B'ar; I sut'n'y glad ter see you dis mawnin'; how you come on? "Whar you done lef" Mistah 'Tarr'pin? Tee-hee! Seem lak he ain' gwine set de evenin' out wid you dis time. Tee-hee!' She titter ag'in an' sort er hide behime her han' lak she feel monst'ous backwu'ds now dat Mistah B'ar 'bout ter win de race.

"B'ar he say, 'Sho! I lef" dat li'l contrapshun down de road so fur aways dat he kain't ketch up in a mont' er Sundays. Him ter race wid me! I tol' him, ma'am, dat dar wuz nuver any queschin who gwine beat. an' dat I knowed you wuz knowin' dat w'en you putt us ter racin'.' B'ar he swelled roun' some mo', an' gal she snort an' snicker some mo', an' las', jes ez de clock 'bout ter strike twelve she git a cheer an' ax him ter set down.

“'Don' krare ef I do, ma'am,' sezee, an' he squared roun' wid his back ter de cheer ter set down. Tarr'pin he tuck dat "casion ter drap down inter de cheer. blip! an' dar he sot lookin' ez peart ez a lizzu'd an' he sings out, 'Hol' up, dar, Mistah B'ar; don' you set down on me, suh. 'kase I kin bite hard, ef I ain' re'y big.'

"B'ar he wuz mo'n half squat down an' he riz up in a hurry an' turnt 'roun' wid his eyes fair' bulgin' outen 


\section{AT THE BIG HOLSE}

his haid, an' sezee, 'Name er common sense! how did you git yer?"

"Tarr"pin say, sezee, 'Nemmine how I got yer; yer I is an "yer I stay. I done tol" you w"en I met up wid you in de road dat de longes' pole reaches de 'simmon, but de smartes' dog gits hit, an' now I done prove de trufe er dat sayin".'

"Gal she say, "Mammy, I wants you jes fer ter look at dat Mistah Tarrpin settin up dar in de cheer ez cool an' comf'ble ez kin be. not a speck er dus' on his footses, an' not even outen bref. He ain' faze' by dat long walk, an' yer Mistah B'ar, big an' strong ez he is, all tuckered out. 'Clar' ef hit don' beat bob-tail! Shows dat you kain't go nuttin' on looks.'

“'Dat you kain't!' sez de Tarr'pin, sezee, 'ner hit don' do ter be sho" ur anything in dish yer mawtul worl'. Nistah B'ar monght a beat me easy "nuff, eren to des de las', ef 'twan't fer dat second nap he done tuck; dar's whar he lose his needle.'

"De gal an' her mammy dey say, 'Now ain' dat de trufe! You sut n'y got good horse-sense. Mistah Tarr"pin, an' we glad you done won de race. I 'spec' you lak mighty well ter wash de dus' outer yo' th'oat wid a gläs er cider atter dat long walk. Jes' set still an' git out yo' pipe an' mek yo'se'f right at home.'

"Dey stood dar muchin' him an waitin' on him, an' dey ain' even ax Mistah B'ar ter have a cheer, so he lef' Tarr'pin settin' dar sippin' de cider an' went off down de road suckin' his thumb all de way an' thinkin' w'at a consumbunkshus fool he done mek uv hisse'f." 


\section{MOLLY COTTON-TAIL STEALS MIR. FOX'S}

\section{BL'TTER}

"Aunt Tancy" said Janey. "do you know any more stories about Mis" Molly Cotton-tail? I think shes most as smart as Mr. Hare, and I like to hear about her most as much."

.. 'Mos' ez smart! 'mos' ez smart! well ef dat don' beat all!." said Nancy. throwing up her hands in affectation of indignant surprise. ". Lemme tell you dat w"en a ooman start out ter be trickish she kin beat a man ev'y time. 'kase her min' done w'k heap faster an' she see all roun' an orer an' m'need an on bofe sides ur a thing wile he stryin" ter styare plumb thu hit."

"Didn't you say she was his wife?" asked Ted. "I should think they would have called her Mrs. Hare alwars, instead of Molly Cotton-tail. Why do you spose they didn't?"

"Law ! honer," she answered. "don' come axin" me no sech li"l fool queschins ez dat. How rou reckon I gwine know all de w's an de wharfo's uv all de ol'-time doin :- I kain t spresify de reasonment ur her bein' call Molly Cotton-tail sometimes, but hit come cross me jes' now dat mebbe de ma'ied wimmins ain' tucken der husban's name in dem dars lak dey does now. Anrhows, I know she called Mis' Molly C'otton-tail, an' not jes' wholly an' solely de wife er Mistah Hyar'. She ain' de 


\section{AT THE BIG HOLSE}

sort er ooman ter sottle down an' be jes' plain Mis' Ilyar all her days, an' stay home an' lissen at de chillen cry an' wash der faces an' comb der hyar an' cook der vittles, ear in an' ear out. Huh-uh! Mis' Molly she got too much git up in her fer dat. She mek ol' man Hyar' stay home an' min' de chillen now an' den, an' he ain dast ter say no, neener. Lemme see, now, whar wuz I at? Ef I'se gwine tell you any mo' tales, you mus'n' come at me dat-a-way wid queschins, lessen you wanster putt de tales outen my haid. I jes' had my mouf fix' ter tell you one w'en y'all bruk in on me 'bout de name. Lessee, wat $u$ ' dat tale 'bout, anyhows?"

"Well, I asked you for another one about Mis' Molly Cotton-tail," said Janey ; "so may be it was about her."

"Sho" nuff, sho" "nuff," said Aunt Nancy. " "Pariently I'se gittin' fibble in de min' ez well ez in de j’ints, ter go fergittin' dat-a-way. Yas, de tale wuz 'bout one time w'en Mis" Molly an' Mistah Fox go ter mek a visit wid Mistah Fox's brer whar live 'cross de swamp an' down in de holler. He wuz right fren'ly wid her 'bout dat time an' 'vite her ter go wid him. She wuz all dress' up in her good clo'es an' her good manners, gwine 'long mekin' husse 'f mighty 'greeable, talkin 'bout dis an' dat, cuttin' her eye up at him real sweet an' stickin' ez clost ez a bur to a cow's tail."

"Why, I didn't suppose they would ever be good friends again after the tricks she played him," said Janey.

"L'm! chil"," she answered, "dis happen so long atter dat ol' Fox plumb fergit dey nver had any fallin's-out. You better not pester me no mo' er I mought fergit de tale, clean ez a whustle. Well, dey went on, him he'pin' 


\section{MIS' IIARE STEALS MR. FON'S BLTTER}

her over de foot-logs. mighty mannerly, an' rumnin' on an crackin jokes wid her, an' las" dey got ter de house. Mistah sly-fox's brer w"ich dey call him 'Hongry Billy' "kase he wuz all time eatin' up folkses chickens, he 'vite 'em in an' tell 'em fer ter mek derse'fs at home an' ax 'em ter stay ez long ez dey kin. Mis' Molly she teck off her hat an shawl an' de ridicule f'um her arm an' lay em on de baid. Den she say. 'Mistah Hongry Billy. I se dat indushous I kain thear ter be idle even w'en I'se off on a risit: so please, suh. ter lemme git de supper. stidder settin' yer hol 'in' my han s.'

"Billy tell her he ain' kvare, so she whu'led in an' set de table an' drord de pine-tag tea an' mate de ash-cakes, an' Hongry Billy he showed her whar ter git de butter. down at de spring. W'iles dey 'z at supper 'long come a naber an' tol: 'em dat ol' Mistah Gray-fox wuz dyin' an' done sont fer Billy ter set up wid him. So Billy scuse hisse'f an' ax 'em fer ter teck kyare er de house ontwel he come back an mek dersefs right at home. Den he $\mathrm{g}^{*}$ long off wid de naber.

"Dat night, w'en Mistah Fox git ter noddin' an" snoogin' by de fire, Mis" Molly she slip out ter de spring an' et up de butter down ter de re 'y las' smidgin, an' den set to an' licked de crock twel twuz clean ez ef hit bin scoured. Den she lick her mouf an' whiskers clean. an' come in an' sot down by de fire ag in befo' Fox had time ter wake up an' miss her. She sot dar lookin' ez innercent ez a lamb, gwine on wid her knittin an' hummin' a chune. jes' ez ef she ain' nurer had butter in her min' ner in her mouf.

'Nex' mawnin' Hongry Billy come back. cross an' sleepy fum de settin'-up. an w'en he go down ter de 242 
spring ter git de butter fer bre"kfus", dar sot de crock, empty ez a go'de. I let you know he wuz mad. IIe come a-huffin' an' a-putfin' up ter de house, an' he say, sezee, 'Dish yer a nice howdy-do! Y'all calls yo-se'f 'spectable folks an' comes yer an' squats down on me, an'

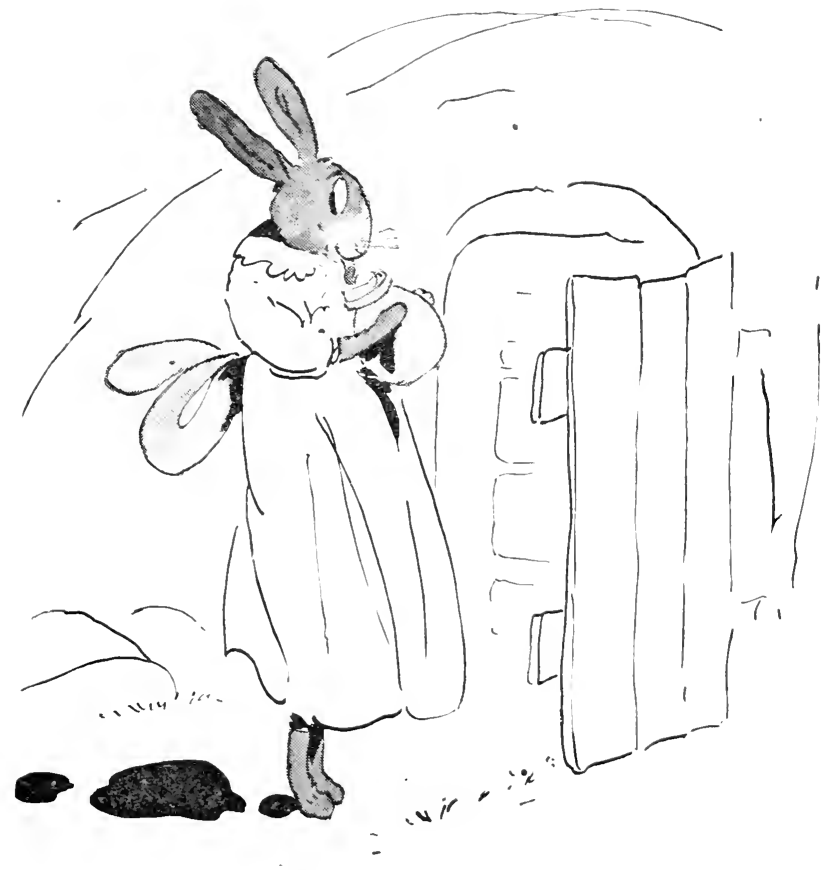

den w'en I turn my back you eats up ev'y rap an' scrap er butter you kin fin' on de place. I bin thinkin' y'all wru folks an' now I fin' you is hogs!' An' wid dat he turnt his back on 'em bofe an' went flouncin' out de do'. “Dey foller 'im up. 'clarin' dey dunno nuttin' 't all $2+8$ 


\section{MIS' HARE STEALS IIR. FON'S BLTTER}

'bout de butter. an Mis' Molly she do talkin' "nuff fer bofe, she do. she sar. sez she. "Twan't me, suh, deed 'twan't! I cross my heart: W'at I want wid yo butter, anyways? I got me plenty butter at home: I ain hatter wo ter de nabers for er y lil ol snack er vittles I want. sidesen dat, I got a mighty delikin stummick. an I kain't eat no butter lesen I done mek hit mysef, kase I ain' sho' de folks bin right clean an kyarful in de mekin.'

"Dat mek IIongry Billy madder'n befo", an' he say. sezee, "Well, mehbe my butter wan't clean: I dumno bout dat, but I does know 'bout dis: hit clean gone, an'w'at's mo; one r you two is de pus-son whar goned hit. I gwine keep you bofe right rer 'twel I fine out wicht is de thief.'

"Molly she study li"l. an' den she say, sez she, 'Mistah Billy. I'se re' saw' dis happen, 'deed I is. But wats done kain 't be ondone, an' so de on 'ies' thing now is ter prove w"icht er we-all tucken yo butter. Ef I ain' 'ceive mysef. I knows de sho' an' sut'n way ter fin' out w'icht is de thief. Tes' you g*long 'bout yo' wu'k an' let me an Mistah Sly-fox lay down yonner in de sun all day, an' w'en you comes back dis evenin' I boun' you kin tell w'icht ur us done et de butter.'

".Huccome?' sez Hongry Billy, sezee.

"Dat easy ez rollin' off a log,' she say. 'De heat er de sun gwine strak thu an' dror de grease out, so t w'en you come back all you hatter do be ter rub yo fingers orer ow' stummicks an' den rou'll know in a jiff who done swollered ro' butter.'

"Mistah Slickry sly-fox 'gree ter dis, 'kase he know he ain't tucken de butter, so he wan't 'feard hit be proved up on him. Hongry Billy he say hit look mighty reas'n- 


\section{AT TIIE BIG HOUSE}

able an' he 'gree ter hit, too, an' went off 'cross de swamp ter tend ter his wu'k, an' lef" 'em bofe dar layin' in de sun.

“Atter w"ile she say, sez she, blinkin' an' battin' her eres lak she kain't keep 'em open, 'T'm-umph! Mistah Fox, dat sum mek me pow'ful sleepy, please 'scuse me, suh, but I'se jes' nachelly 'bleeged ter teck a li'l nap.' Fox say he b'lieve he teck one hisse'f, an' he shet his eyes an' putt his nose down between bofe jaws an' gin his tail a whisp er two ter drive 'way de flies, an' pres'n'y he wuz fas' asleep.

"Mis' Molly she watch him outer de cornder uv her ere, an' w'en she see he wuz good an' soun' she lit up f'um dat widout mekin' no noise an' struck out fer a naber's spring an' got her a han'ful er butter. She come tiptoein' back an' stoop down an' rub de butter all over Mistah Fox's stummick, so sof'ly dat he ain' kin feel hit, an' den she go an' lick her paws clean an' laid down in her place ag'in an' kep' one eve on him. 'Bout de time w'en she 'spec' Hongry Billy home she shet her eyes an' snore so loud he year 'er clean 'cross de holler. W'en he come up to 'em dey wuz 'pariently fas' asleep, an' Billy he retcht down an' run his han' over Mis' Molly's stummick. Dry ez a bone! Den he try Mistah Slickry Sly-fox, an' he bringed up his han' kivered wid grease an' smellin' loud er butter.

"Billy wuz in a turr'bl' teckin' an' mek sech a fuss dat he wake Mistah Fox an' fall ter excusin' him er teckin' de butter. Ol' Fox wuz dat s'prise' ter fin' his stummick kivered wid butter dat he ain' kin say nuttin' 't all. 'Bout den Mis' Molly Cotton-tail 'gun ter stretch an' 


\section{MIS' HARE STEALS MIR. FOX'S BUTTER}

rub her eves an' 'tend lak she jes' wake up. She lissen at all de gwines-on a minnit an' den she mence ter mek gret 'miration. 'Laws-a-musy!' she say, 'I ain' b'lieve dis ef I ain' see hit wid my own eyes. I sut'n y is scannelize' dat a fren' er mine tucken ter stealin'. mo' special on a risit. I 'clar' ter goodness my feelin's is so hurted dat I kain't rightly 'spress myèf, an' w'at's mo'. I kain`t stay yer sociatin' wid a common thief. I got my young fambly ter think bout. So long, Mistah Hongry Billy. I hopes you gin dat man a good walluppin', 'kase he sutn'y arn hit. comin' rer puttin' up on you an' den cleanin' out yo' butter. Nex' time you git me ter go risitin' wid you, Mistah slickry sly-fox, you be heap older an' smarter dan w'at you is dis minnit.' Wid dat she go scootin'. 'kase she 'feard ef she stay longer she mought git foun' ont somehow er nùr.

"Hongry Billy he study li"l w"ile an" den he say ter Mistah Fox, 'Mell, I gwine let you off dis time, kase you is my kin an' I ain' wanter disgrace you; I ain' wanter let folks know I got a thief fer a brer. But don' let me ketch you in dese diggin's no mo', er I gwine set ter w'k an' lam de butter onter dat greasy hide er yo'n: you year me talkin"."

"Fox he 'clar" an" he sw'ar dat he wan't de thicf. but Billy ain' pay no 'tention to him. Den he had half a min' ter tell ol' Hongry Billy 'bout all dem henroos'es he done robbed. but Billy so mad a raidy dat he kind er 'feared ter do hit, so he go slinkin' off wid his years down an' his tail draggin', an' dey tells me dar wuz a gre't coolness spring up in dat fambly dat las' fer some ser'l 'ears, all 'longer dat crock er butter. I let 


\section{AT THE BIG HOTSE}

you know, chillen, hit ain' teck much ter start a fambly juoi'l, but hit teck heap er time an' trouble ter patch one up, jes' de same ez hit do wid dem holes whar li'l Mars' Ted snag in his britches, an' nuver seem de same atter de patchin', neener." 


\section{THE LAZY FOX}

When Aunt Naney finished the story of the stealing of Hungry Billy"s butter, Janey said: "I should think Mr. Fox would have been so mad at Molly Hare that he would never have spoken to her again. Didn't he ever try to pay her back :"

"Deed dat he dir." said Aunt Naney, "an" he come mighty nigh doin" hit, too. Dish yer wuz de way ur hit. One day he wuz turr"bl' hongry, "kase his brer Billy bin cleanin' out all de hen-roos'es in dat naberhoods 'twel dar wuz no chickins lef" fer him; he kain't tell whar de nex' meal er vittles gwine come f"um. Las" he say he gotter have sumpin ter stay hisece wid an he b"lieve he lak mighty well ter have a hrar. But he wuz so mawtul lazy dat he ain wanter chase one down, kase w'en de foxes chases de hyar's dey hatter do a pow'ful lot er rumnin' befo' dey kin git 'em. Fox he knowed dat; so he go walkin' 'long thu de woods. schemin' away ter hisse'f an tryin' ter mek up a plan ter ketch a hyar' widout chasin' her. 'Dar's some way ter do hit, sho' ez I'm a livin' simner, an' I gwine wu'k my thinker 'twel I fin' hit out, dat I is.' sezee. 'T'm one' $\mathrm{r}$ dese yer thinkin' men.' sezee, winkin' up his eyebrows, 'an' w'en I putts my min' on a thing hit. ez good ez done.'

"Jes' den he come 'cross one'r dem pafs whar de hyar's use w'en dey track up an' down thu de woods, an'

$$
253
$$




\section{AT THE BIG HOLSE}

sezee, 'Heyo! yer's a hyar's run. I'll jes' foller dis up an see w'at I kin see. I boun’ some er dem fool creeturs gwine be 'long yer betwix' daylight an' dark huntin' fer rittles, er gwine down ter de branch, er was in' der time gwine roun' visitin'. I lay I'll fix up some scheme ter ketch one ur 'em.'

"He g'long moufin' 'way ter hisse"f, an' all ter onct, in de middle er de paf, he seed one'r dem 'ar holes full er mud whar dey calls 'slashes,' an' w'en he see dat he say, 'Now yer's whar I gwine lay low an' ketch me a hyar fer my dimner.' So wid dat he got down inter de slash an' stood dar up ter his neck in mud, waitin' an' waitin'.

"Pres'n'y he year a noise an' look up, an' lo, beholst, some un wuz comin' down de paf, an' 'twuz nob'dy mo' ner less dan Mis' Molly Cotton-tail husse'f, singin' dis song at de top ur her voice:

'Ez I went dou'n de new-cut road, I met de tap an' den de toad;

De toad he'gun ter whustle an' sing, An' de 'possum cut de pigeon-u'ing.

'Long come an ol' man ridin' by:

"Ol' man, ef you don' min', yo' hoss'll die."

"Ef he dies I'll tan his shin, An' ef he lives I'll ride him ag'in.",

Fox he lay back his years an' wait, not even so much ez winkin' a eyeled. He wait 'twel Mis' Molly git right 'long side ur "im an' den sezee, in de mos' pitifules' roice he kin putt up. 'Mis' Hyar"! Oh. Mis' Hyar'! Please, Mis' Hyar'! Oh, stop ; stop. Mis' Hyar!' 


\section{THE L.AZY FOX}

"Mis' Hyar" she shied clean ter tu'rr side de road w'en she rear dat noise an" pricked up her years an" stopped singin "right in de middle ur a w'd, an" sez she. "W"at?" Den she look roun' an' see him lookin' lak he wuz stuck fas" in de mud.

"He go on in dat same pitiful roice, 'Oh, Mis' Hyar', I'se in de mos'es trouble l urer wuz in my life.'

"Sho"! look ter me lak you'se in mud,' sez she wid a snicker. ' $\mathrm{C} r$ all de notions! W'at in de worl' mek you wanter res" yo"se"f in dat slash w"en you got all de dry lan ter lay yo"se down on" Mighty cur'ous tas"e you got, Mistah Fox," sez she wid anu rr snicker, "kase she thought he wuz sho"'nuff stuck fas" in de mud an' de notion tickled her might"ly.

" 'Naw'm. I'se in sho"'nuff trouble: sezee. 'Please, ma'am. don' you laugh at me. Please fer ter he"p me out ef you kin.'

"Sez she: "G"way fum rer. man: you talk plumb foolish. You spose I gwine hep you out w'en I bin yearin all my life dat foxes eats hyar: Naw siree bob! I ain' ro" fool dis time.'

"Fox he say, 'Don' let dat ol' sayin' worry you, ma'am.'

"She say: "I dunno bout dat. Better worry now dan atter you done ketched me.'

"He go on baiggin". Sezee, "Mis" Hrar", ef rou'll on'y jes" he'p me dis onct I won' ax you ag"in, an' I gin you my promuss nurer ter fergit hit.'

"Mis" Hyar" she look at him sort er jubons an" sturly 'bont hit a w'ile. Las' she say: 'I gwine tell you now dat ef I do he'p you, you mus'n fergit hit. I gwine 'pend on you fer dat.' 


\section{AT TIIE BIG HOLSE}

"He say. sezee, "Deed, dat I won'. Mis' Molly; you shell alluz have de praises: dat you shell.'

"Den she mek up her min' ter he'p him, 'kase she feel right saw fer him: so she step up in han'reach uv him an gin him her paw.

"He wuz plumb tickelt ter def by dat, an' hit did him so much good dat he drapt his rears back an' tended lak he wuz in a wuss fix dan befo'. He say: 'Oh me, oh my! Mis Hrar'. I'se so weak. seem lak I ain' gwine be able ter pull outer yer. Th' gimme all yo' strenk! squnceze tight! Lay back on me, madam, pull! pull hard! uh-huh!" Wid dat he putt one foot out an' den tu'r an' den "tended lak he z gwine fall in ag"in. Mis" Hyar" she putt on sech a extry bus" er strenk dat hit come nigh bein' de def ur her, an ' lan 'ed him 'long side her on de dry grom?.

"She say. w" she cu'd git her bref 'nuff ter talk: 'Now, Mistah Slickry sly-fox, I hope you gwine keep outer sech messes atter dis, er else not call on me, fer I nigh bout teetotally ruint myse"f he pin' you outer de fix. 'Tain' sprise me ef I nuver git orer dis, sez she, hol 'in' onter her side an' jumpin' ev'y now an' den an' vellin' 'ouch!' w'en a stitch tuck her suddintly.

"Fox he wuz drippin' wid mud an' shakin' an' shirerin' f'um bein' wet so long, an' Nis" Molly say. 'You bes" go right 'long home. now, an' git ro'se'f good an' dry er nex' news you know you be havin' de ager.' She say dat 'kase she gittin' kind er anxious ter git shed ur him, she ain' lak de look ur his eye w'en he wall hit roun' at her. He ain' budge w'en she talk 'bout him goin' home, so she mek has'e ter say, 'You know w'at 


\section{THE LAZT FOX}

you done promuss, suh: you done gin me yo' wu'd dat you ain gwine fergit w'at I done fer rou.'

"He laugh an' gin hissef a shake lak de dogs does w'en dey bin in de water. an' sezee: 'Dasso, Mis' Molly. dasso: I done promuss you an' I mighty saw'y fer hit, too: "kase right yer"s whar I gotter break my wud. Hit goes hard wid me ter do it. but wen a man"s right hongry. wat kin he do? You know how tis yo"se"f. Mis" Molly: w'en rou git good an' hongry you don' stop ter think "bout de peas in de naber"s gyardin' not blongin to you; now, does you? 'Clar" ter gracious. Mis' Molly. you kain't 'spec' me ter have sech luck ez lis an den turn hit a-loose. now. kin rou?"

"Mis" Molly she tuck a li"l step ter one side an' thowed back her haid an looked him in de eve right sorrowful, an' sez she: 'Deed, Mistah Fox. I didn' think rou'd disapp "int me dis-a-way: deed I didn'. Please, suh, ter turn me a-loose.'

"Fox he kep" tight hol' ur her paw an' tuck a step w'en she did. an' sezee. 'Ha! ha!' ha!.! I reckon not. ma'am : not w“iles I'se in my seren senses.'

"She plead an" she plead an" she baig an" she baig an" she argyfy an' she argyfy wid him. but he ain' pay no 'tention to hit, an' he kep' sech a tight hol' on her paw dat she ain' see no chanct er breakin' 'way. So las" she let on ter gin up an' she say: "Tell. Mistah Fox, I done gin up. I see you is 'termine' fer ter kill an' eat me. an' I done mek up my min' ter die. I know you 'spec's ter kill me, but befo' you does dat. I got one li'l faver ter ax you. Wistah Slickry: jes' one li'l faver."

" "W"at's dat?' sezee. 'Lemme rear hit an' mebbe I $25 \%$ 


\section{AT TIIE BIG IIOUSE}

mought grant hit, scein' dat you is so near de een' er ro' tether. Dyin' folks mos' alluz gits der las' reques'es granted; so speak up, ma’am, fer dish yer’s yo' las' chanct.'

'All de time dey bin talkin' an' argyfyin', Mis' Hyar' she bin aidgin' closeter an' closeter ter de briers, an' w'en she git right elost she say, 'Mistah Fox, you sut'n'y is kin' ter grant my reques'; thanky, suh; thanky, thanky.'

“. 'Temmine ro' manners,' sezee; 'T'se in has'e. Jes' spit hit right out an' le's have hit over.'

". 'Tell, den,' she sez, 'I is a ooman; you knows dat; an' wimmins is alluz bein' excused er cur'osity, an' I 'spec' I has my sheer, fer I bin wunnerin' all my life ef hit's true dat foxes eats hyar's, er does dey jes' chase 'em an' den larrup 'em good, ef dey ketch 'em, an' den let 'em go. I never bin lietched befo', so I dunno how dey does. I thought mebbe de on'ies' reason you wuz gwine kill me wuz bekase I done trick you some few times. Secm lak I mought die mo' easy ef I knowed jes' 'zackly how 'tis in dis marter. Now, ef de foxes does mek de practuss er killin' an' eatin' hyar's, will you please, suh, ter onloose yo' right han' fer a minnit an' lif' hit up an' 'clar' an' sw'ar dat you gwine kill an' eat me an' ez many mo' ez you kin ketch?'

"Fox he langh an' he laugh, an' he say: 'Lawdy, Mis' Molly, you bin livin' on top de yearf all dese 'ears widout l'arnin' dat foxes ketches an' eats hyar's w'ensomurer an' howsomurer dey kin? I sut'n'y is s'prise'; I thought you'se smarter dan dat.'

"'I done year tell ur hit,' sez she, 'but hit seem too 


\section{THE LAZY FOX}

turrbl' ter be true: so please, suh. I'd lak ter know de sho"-nuff fac"s in de case befo" I die."

"Dat you shell," sez Fox. sezee. an" wid dat he let go Mis" Molly"s paw an' raise his han' up. an' sezee, 'I do rerby "clar" an" sw"ar ter kill an" eat Mis" Molly Cotton-tail an ez many mo' ur her lin' ez I kin lay my han's on, so-he p-me-bob!'

"Mis" Molly ain" stay ter year him out. De minnit he onloose her han', she jes' slip" inter de briers so quick dat Fox ain see whar she went to. He stan dar strarin 'fus" dis-a-way an" den dat, too pa'lyze' even ter bring his han down by his side ag"in.

"Mis' Molly, w" en she git good an' in, whar she feel right safe, she stan" dar an" sass him. She say: 'Come on inter de briers. Mistah Fox, an' git me. Come on an` git dat fine bushy tail er ro'n all tankle" up wid de briers. W"at mek you wait? You ain" use to 'em lak me, 'kase yer whar I spen' mos' er my time, an yer whar I 'spec's ter die: not in yo' han's, suh: you nuver gwine git dat chanct ag "in. Thanky, suh. fer grantin' dat reques' er mine, dough I'se kind er 'feard hit's sp'iled yo" dinner. I gwine roun' tell all de nabers how kin ' rou wuz, an' I gwine send noration roun' dat de trufe done bin foun' out at las": fores does kill an' eat hyar's, sho" 'nuff-dat is. w'en dey kin ketch 'em.'

"Irid dat she go scurryin" home thu de bresh. an' she sez ter husse'f, she sez: 'Molly Hyar'. yer's whar you done mek a plumb fool er yo'se'f an' come mighty nigh kickin' de bucket fer yo' pains. I lay you "ll have mo" sense nex' time dan ter git ketched in any sech traps ez dat you jes' tucken yo' foot out ur.'" 


\section{MR. IIARE, MIR. IIINK AND MIS' DUCK}

"Aunt "Phrony," said Ned one day, "do you know any stories about Mis" Duck?"

"Ir"at mek rou think "bout her?" she asked.

"Oh." said he. "I saw her waddlin' roun' the yard the other day and I wondered why you had nerer told us any story about her."

"Sho! chil"," said she, "w"at ynu reckon I wanter study "bout dat li"l ol no-kyount quackin" fool fer? I'se gittin' too ol' ter let my min" run on "bout sech foolishness ez dem ol' tales. anyhows. Me wid one foot on dry lan' an' one in de grave: I orter be thinkin' 'bout dish yer urerlas"in' soul er mine er'y minnit I ain' hatter think 'bout meat an' drink an' kirerin' fer dis po' ol' pe "ishin "body er mine."

"Oh," said Ned apologetically, "I never thought about its being foolishness. I like your stories a lot. and I know there are not many people can tell them the way you can, unless it's Aunt Nancy."

It was nice of Ned to be loyal to the absent Aunt Nancy, but her name at once ruffled Aunt 'Phrony, for she went on: "I reckon you mought know "twuz foolish- . ness waturer dat ooman tell you. sidesen dat, knowin" is one thing an' tellin's anu'rr. Mebbe I knows tales 'bout Mis' Duck. but mebbe I ain' think hit wuf de w'ile ter tell em."

The children proceeded to offer bribes with a view 260 


\section{MIR. HARE, MIR. MINK AND MIIS" DTCK}

to making it worth Aunt 'Phrony's while. Taney produced the battered brass thimble with which she was wont to force a great spike of a needle through her dollrags. Ned offered his best "agate," and little Kit offered a kiss as the only marketable thing in his possession. The kiss carried the day. and after the old woman had filled and lighted her pipe and taken a few preliminary puffs, she began:

"One time Mistah Hyar' met up wid Mistah Mink an he putt out his paw an' she han's an' made hissef monstous fren'ly, an done lak he thought Mink was a mighty nice sort er feller. but all de time he wuz sarchin' rom' in his min ter fin' some way er ru'r ter show his own se'f off an let Mistah Mink know how smart he wuz. kase he wan't nuver sassified lesen he let people know 'bout dat. Las' he say: 'Mistah Mink, dish yer mighty fine day; le's we-all teck a li'l walk toge'rr. S'posen we saschay roun by de pond.'

"Mink he say he jes' ez lief go dat-a-way, ef not ru'r, 'kase de pond seem mighty home-lak ter him: so dey walk on an' dey walk on an' las dey git ter de pond an' dar wuz a nice big flock er ducks floatin' roun' in de water, so still dat hit seem lak dey ain stirrin'. Dey look lak deỹz so lazy dat twuz come-day-go-day wid 'em: rou oon a-knowed dey wuz scrabblin' roun' fer der vittles 'lessen some un tol' you.

"Hyar' he watch 'em a w'ile an' den he say: 'Mistah Mink.' sezee. 'I don' wanter show too much cur'osity, suh. but jes "twix" fren" an' fren' I lak ter ax you how you gits yo livin": I see you lookin right plump an' peart fer a man er yo build, an I lak ter know de w'y an' de wharfo's.' 


\section{AT THE BIG HOLSE}

“' $\mathrm{W}^{\prime} \mathrm{Y}$, sut'n'y' sez de Mink, sezee: 'I gits my livin' right outer dat pond widout no trouble th all. $\|^{\prime \prime}$ en I wants me sump'n ter eat, I jes' whu'l right in an' git me a duck.'

"Hyar" wuz sniffin' away ter hisse'f, kinder scawnful, but he say ter Mink, mighty p'litely, he do: 'You don' sesso! Well, Mistah Mink, I lak ter know jes" how you do dat. Please. suh. ter show me how you ketch duck; I bin yearin' all my days w'at a gre't han' you is at duckketchin.

"Dat mek Mink feel kind er good. an' he say, sezee, 'Tell. den. jes' watch me clost, an' I reckon mebbe you'll be able ter do it in de same lak manner.'

"Mink he slipped inter de water an" dore clean unner ter whar de ducks wuz a-floatin', an' dey ain' even know he wuz near 'em. Den he ketched one ur 'em by de laig an' jes" natchelly pulled her un'need de water an' drug her orer ter whar Mistah Hyar" wuz waitin'.

" "Ch-huh, Mistah Mink." sezee, "re"Y good; you has did dat mighty slick. suh : but I boun' you I kin do dat same de ve'y fus' lick widout de practuss you bin harin' all yo" days. De on "ies" thing needed yer is ter be handy an 'quick. suh; handy an quick;' an' he twis' up de cornders ur his mufstarsh wid one han' an' look mighty knowin'.

"'All right, sulh,' sez Mink, sezee; 'sail in an' lemme see how you gwine do de deed,' sezee. 'Mebbe you kin do hit all right de fus' time,' sezee, 'but I don' b'lieve in none er dese yer nigh-cuts, myse'f. My spe'yunce is dat you hatter go roun' de long way ef you wanter do a thing well.'

"Dar wuz a log layin" in de water nigh ter whar de 262 


\section{MIR. HARE, IIR. MINK AND MIIS' DUCK}

ducks wuz, an' Hyar' he mosey roun' ter de place an' wade out inter de water an' clum up on de log, thinkin' he'd stan' dar an' grab fer a duck dat-a-way. But dat de time his gun miss fire. He nab at de neares" duck, but sho! he los" his baluns an' fell inter de water kersplash! an’ come mighty nigh gittin' drownded. De ducks dey yearn de noise an' hollered an' quacked an' flew'd off ter tu'rr side de pond. One'r de young ducks whar wuz jes' out fer her fus' swim, she say ter one'r de ol' uns: 'Quack, quack, quack! Mis' Duck, dat wuz a gre't splash we done yearn. Mus' a bin a mighty big frog jumped inter de pond.' 'Lmph,' sez de ol' lady, 'frog! I say frog! You gotter larn dat frogs don' wear fur er you'll nuver have no peace ner comfu't in dis pond. Dunno frogs yit w'en you see 'em, an' gotter mek yo' livin' frog-huntin'! I lak dat!"

“'Tellum,' sez de young un. 'dis my fus' trip ter de pond; you kain't 'spec' me ter know all you does, in a minnit.'

"Dat sort er please' de ol' un, so she stop jawin' an' dey all went on wid der frog-huntin'.

"Hyar' he clim' up de bank mo' daid dan alive, wid ev'y hya'r stickin' ter his hide and de water rumnin' offen him lak rain. He wuz right aggervex', 'kase hyar's don' lak ter wet der footses none too well. He gin hisse'f a shake an' he sez, sezee, 'Shucks! dem de triflines' ducks I urer see, frightened ef a man sheks his li"l finger at 'em; an' dat ol' log so slipp'y you hatter have claws ef you wanter hol' onter hit.'

" 'Temmine. Mistah Hyar', sez de Mink. sezee, 'you mus' try yo' luck ag'in. You mustn' gin up on de fus' try. I gwine swim roun' on tu'rr side de ducks an' 263 


\section{AT TIIE BIG IIOLSE}

drive de hull cumboodle back dis side, an' you mus' stan raidy ter ketch one, fer I boun' you shell have duck fer yo" "linner."

"Co"se Ilyar" wan"t giwine let on he"z stumped, so he say sort er krar"less: 'Oh well. so he ef rou sesso, 'tain' gwine faze me ter ketch one "r dem li'l no-kyount ducks; me dat bin hoein my own row urer sence I wuz kneehigh to a hopper-grass. I gwine nab her dis time, you kin lemme 'lone fer dat.'

"Mink he swimmed off, an" Rabbit he go up on de bank an" git him a long piece er dat brambly rime dey calls de debil's-shoe-string. Well, he tuck an' tuck de piece er debil'-shoe-string an crope down inter de water wid hit 'twel he wuz hidden. all but his nose an' eyes. Ter come ol' Mink drivin' de ducks dat way. One un 'em come sailin' to'des Mistah Hyar' an' he jes' natchelly grabbed her by de laig an' hilt her w'ile he tied her laig wid de debils-shoe-string. she quacked an she hollered an "she flapped an she flew'd, but yit ol Hyar' hung onter de lyrier. "Xo use ter kick, ma'am.' sezee. 'Kase I done got you dis time an' I gwine hol" onter you."

"'T'm-umph! I gwine show you huccome,'sez Duck, sez she, an she flew'd up an' she flew'd up, an' Hyar' went spinnin" an" whu' lin' an" kickin", yit he ain' let go. Mink he wuz stamnin down below jes" a-hollerin' an' a-langhin'. clappin his han's on his knees an doublin' hisceff up lak a jack-knife. He sing ont: 'Hi-ri! Mistah Hyar', you done got up in de worl'. sho' 'nuff. You bin right uppish befo" now; I don' reckon you gwine speak wid common folks atter dis.' 


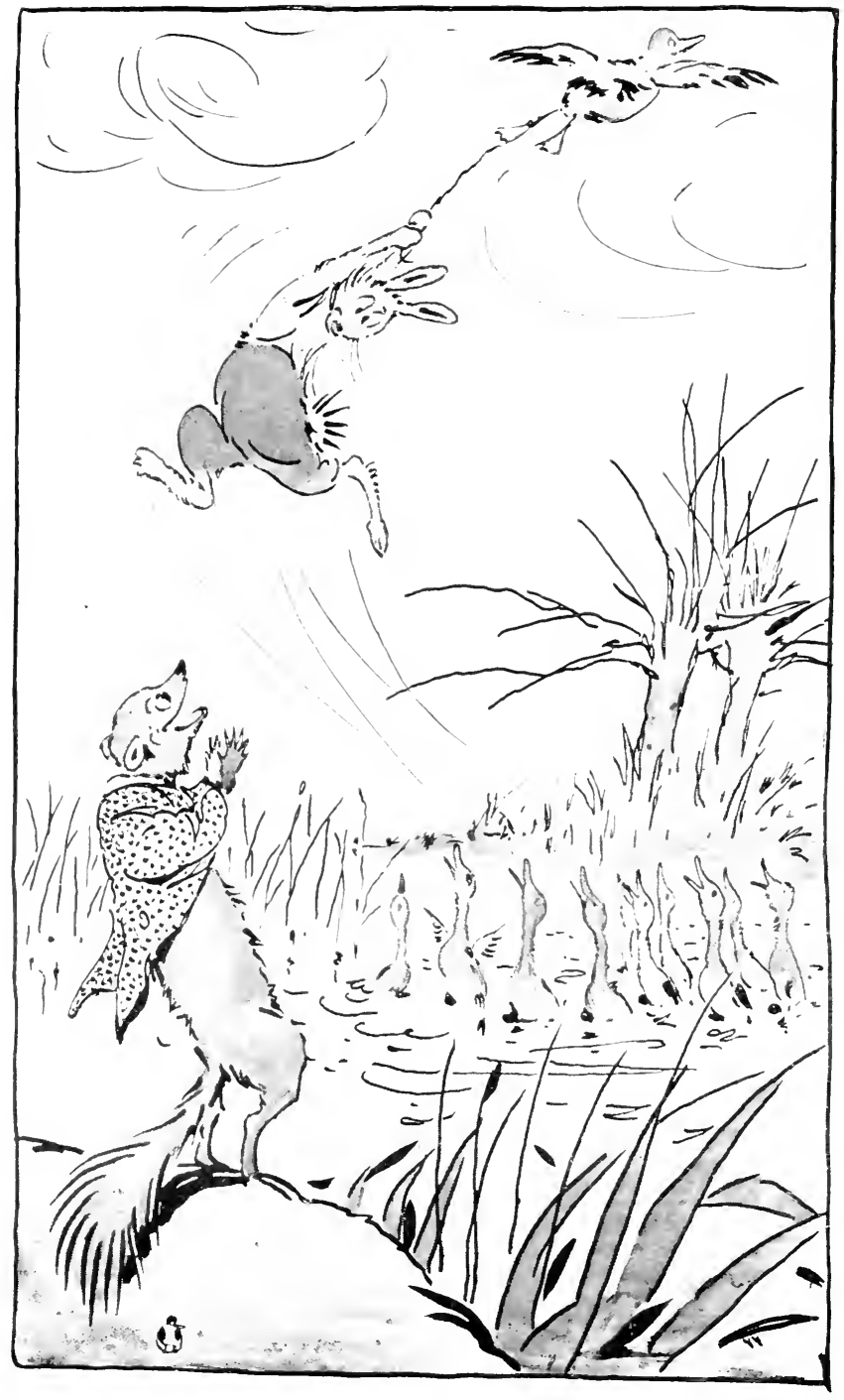

HYAR' WEXT SPINXIN' AN' WHU'LIN' AN' KICKIN' 



\section{MR. IIARE, MIR, IINK AND MIS' DECK}

"I"ll speak wid you w"en I git hack f"um dis trip: I

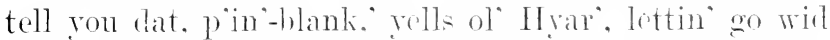
one han long "muff ter shek his fis" at Mistah Mink. Mink he stood watchin" "en "twel der wan't nuttin" but a speck. Duck she flew't an" the flew'id an" the flew'd, an' Hyar' hilt on 'twel he thought his pars grwine drap' off. He argyfy wid her an baig an baig her please, ma'am, ter light an' let him go: but the jes" quack an' say: 'Kain't year you, suh; kain't year you. You hatter speak louder, my years ain' w'at dey necter wuz.'

"Las" he culdden hang on no longer an" he seed dey wuz right orer a ol" sickrmo" tree, an' he let go an' drapt onter de tree. But de tree wuz holler an' he jes' drapt right down eenside "twel he struck de groun'. He wuz plumb stunded at fus: but atter w'ile he set up an' look' roun'. 'Twuz mighty dark in dar an' he sarch an' he sarch fer some place ter git out, but 'twan't none dar. He lissen an' he liseen an' he feel an' he feel. but dar he wuz, shet up tight wid nuttin' but a speck er daylight, way up in de tree.

"Las" he yearn a man choppin" in de woods an' he say ter hissef dat he mus" "trac" de "tention er dat man ef he wanter git out. 'Hi-yi! Mistah Man.' sezee, speakin' fine lak a ooman, 'come yer, Mistah Man.'

"Mistah Man ain' year him an' go on wid de choppin'. " "Tan mus' be deaf ez a pos", sez II yar" ter hisse'f. 'I reckon I gotter yell loud 'nuff ter bus' my b'iler ef I wanter git outer dis.' He try hit ag"in. but vit de man ain' year. Den he thought mebbe de man monght rear him ef he sung sump'n, so he struck up a ol' chune an' dese wuz de wu'ds whar he putt wid hit: 


\title{
AT THE BIG HOLSE
}

\author{
'Oh! ef I had a needle, \\ Ez fine ez I cu'd sew, \\ I'd stitch my true lore to my side \\ An' down de river I'd go.'
}

He made his roice jes' lak one'r dese yer high, fine, ladiz' voices whar carry de soun' a long ways, an' las' Mistah Man year him an' he say, 'Mussy me! w'at dat? 'Pears ter me I year a ooman gwine on inside dat tree.'

"He walk up an' lean his haid 'gins' de tree ter lissen. Den Hyar' he 'gun ter holler an' cry jes' lak a ooman. He say: "Oh, please, Mistah Man, fer ter teck yo' ax an' chop a hole in dis tree an' lemme out. You done tol' de trufe; I is a ooman, an' a pooty one inter de bargum; an' ef you let me out I gwine set down 'long side er you an' let you look at me all you wanter.'

"'All right,' sez de man, sezee, grinnin' ter hisseff; 'kase all mens is mo' er less ur fools w'en hit come ter de marter uv a good-lookin' gal, an' he chop an' he chop, jes' mo 'n mekin' de chips fly in his has'e ter git a sight er de gal. Hyar' he wuz scrouched down in a cornder outer de way er de ax, an' soon ez de hole wuz big 'nuff he tuck an' tuck a good raidy an' went scootin' out, right 'twix' de man's laigs, inter de bresh. 'Hi-yi! Mistah Man,' he yell, 'ain' I de fine shape ur a gal? You kin have me ef you kin ketch me!' an' wid dat he wuz off, singin':

'Oh! ef I had a needle,

Ez fine ez I cu'd sew,

I'd stitch my true love to my side

An' down de river I'd go.' 


\section{MR. HARE, MIR. MINK AND MIIS' DUCK}

"Itrar' he done got so hongry in de tree dat he 'bleeged ter gnor sump'n, an' dar wan't nuttin' else, so he jes' natchelly turnt to an' et his own tail off ; an' dat huccome hyar's bin gwine roun' wid shawt tails ontwel dis day."

"But," objected Ned, "I thought that old Hare was dead. You told us that they killed him by shooting him in the paw, and that they punished the chick-a-dee for bad advice by cutting the end of his tongue off, because he didn't tell them at first to shoot the Hare in the paw."

Aunt 'Phrony was not too well pleased at being called to account for resurrecting her hero, but she condescended to explain: "W" $y$, kain't you see dat dis yer happen long befo' de time w'en he got shooted in de paw? Dat's plain ez de nose on yo' face. 'Kase I tell you dat tale fus', hit's no sign dat hit happen fus'. I'se gittin' so ol' an' rambly dat you hatter let me go back on my tracks now an' den, ef you wants me ter go on wid de tale-tellin'." 


\section{THE HLMIIXTG-BIRD AND THE TOBACCO}

After Aunt Phrony had finished the last story, she sat puffing vigorously away at her pipe, apparently forgetful of her young risitors. They watehed her with expectant eyes, waiting for the moment when she should have had her smoke out and be ready to tell them another story. The small boy eyed the pipe and the old woman with much curiosity, for he had nerer been able to understand the exact relations of the two, nor how so much smoke could pass through her nostrils without her catching fire. Now and then she blew the smoke out in the form of a ring. to the great admiration of the little boy. At last he made bold to ask, "Aunt 'Phrony, why does you like to smoke?"

She ered him with some amuscment and much indulgence in her expresion. "Well, my baby," she said, "w'y does you lak ter eat vittles?"

This was a poser to the little boy, who, conscions of a preference for "vittles," was quite mable to explain it.

The old woman, pursuing her adrantage, went on: "Tou kain't arnser me dat, kin you? To be sho' you kaint. Well, now, I girine tell you, honey; I laks ter smoke "kase I craves hit, an' I craves hit "kase my daddy an' all his folks befo" him bin crarin' 'bacey an' smokin' hit mo' ears dan you has hya's on yo" li'l haid. De Injuns wuz gre’t folks fer smokin”, an`I done year my dad- 


\section{THE HLMAIXG-BIRD AND TIE TOBACCO}

dy say dat white folks wan't knowin" nuttin "bout "bacey ttrel der camed ter dis kyountry an seed de Injuns smokin" hit. Dey smoked w'-n dey wuz tired an dey smoked w'en dey wuz hongry an dey smoked at de kyouncil, an dey moked wat dey called de "pipe er peace' w'en dey wuz makin" hit up wid der inimies. an' dey eren smoked wen dey wuz samblin, fer de Injuns is gre"t gamblers an has der gamblin games same ez de white folks. Deed dey has wat dey call 'gamblin' pipes"; I seed one onct. an" "twuz a mighty cute li"l contrapshun, made outer clay an lonkin sump'n lak a li'l kittle settin "up on thee feet. an" hit have six holes in hit fer de pipe-stems. I 'low dat dey weter sot hit on de groun an' den set roun' hit. an dat de pipe-stems wuz mighty long an' dey all puffed away toge rer widout havin' ter 'tend ter der pipes; an' I 'spec' mebbe 'twuz some sarin" on de "bacey, too.

Daddy tol" me his people had two sawts er 'baccy: de common kin' whar er 'yb'dy smoke-dat sawt have a long leaf: an' de sacred baccy whar dey smoke w'en dey wanter skeer away de witches-dat kin' hare a rom' leaf. De Injuns b lieve in witches an' ghos'es, an' dey think de ghos"es comes back an meks der kin-folks dream 'bout 'em, an 'witches 'em dat-a-way. an' dey think ef sump'n ain' done 'bout hit, de pusson girine die. Dey calls dem ghos'es 'night comers' an' ter git shed ur one. de pusson whar done bin "witched tecks some er de sacred "haccy an' smokes hit. sayin' orer some w'ds. an" de smoke meks de ghos" cough an' jes" chokes him ter def. so he kain't come back no mo'.

"Daddy say one time de "baccy wuz tonken "way f"um de people, an' dey git jes' fair' faint fer de want ur hit. 


\section{AT THE BIG HOLSE}

Las' dey foun' out 'twuz kep' in a place whar dey kain't git at hit. an' wuz gyarded clost by some turr'bl' thing er ru 'rr, dongh dey kain't fin' out jes' w'at 'twuz. Dey called a kyouncil toge'rr an' 'cided ter sen' one'r de creeturs atter de 'baccy; so dey pick' out one dat wuz a mighty swif' trabeler 'pun de groun', an' dey tell 'im he mus' mek gre't has'e, 'kase dey oon be able ter keep up no sech a honin' ez dat wuz much longer.

"He g"long an' he trabel an' he trabel an' de people dey wait an' dey wait, but he ain' nurer come back. By dat time dey wuz gwine roun' wid der empty pipes in der moufs, puffin' 'way ter keep up der sperrits. Den dey tuck an' sont fo'th nu'rr creetur whar trabel 'pun de groun', mo' swifter dan de fus' one, an' he ain' come back, neener. Den dey sont mo' an' mo' er dat 'ar kin', an' none ur 'em camed back; so las' dey see dat ain' gwine do.

"Den de cunjerers dey say : 'Le's we-all call up dat wise man, Mistah IIole, whar trabel un'need de groun', an' see w'at he kin do fer us.' So dey call Mistah Mole up f'um de groun', 'kase he wuz 'bleeged ter come at re spell er de cunjerers, an' he 'greed ter unnerteck de bizness, dough he knowed 'twuz mighty dangersome an' dat he wuz a fibble pusson an' blin' inter de bargum. Yit dis is w'at he thought ter hisse'f: 'T'll jes' keep scoopin' 'long un'need de groun' whar no pusson kain't see me, an' w'en I git ter de place whar de 'baccy is, I'll jes' natchelly retch one paw outen de groun' an' snatch de seed an' go back de way I come, an' den de people kin plant de seed an' grow all de 'baccy dey wanter.'

"Mistah Mole he start out on his trip, pryin' an' prizin' up de groun' ez he go, an' scoopin' an' shovelin' 


\section{THE HLMIING-BIRD AND THE TOBACCO}

wid dem li'l paws er his'n. Ias' he git mighty tired an' he say ter hiscef, 'Mussy sakes!' sezee, 'yer I bin diggin' an' scratchin' wid my han's an' pryin ' an' prizin' wid my nose all dis time; "pears lak I orter be nigh dat 'baccy; I gwine poke my paw out an' feel.' Wid dat he up an' stuck one paw thu de groun' an' suz! de Thunder an' Lightnin' whar wuz grardin' de 'baccy, dey jes' up an' hit him a clip an' dar he wuz, strotch out daider'n a do"nail.

"De kyouncil dey waited an' dey waited, an' de people dey waited an' dey waited, but Mistah Mole ain' come back. Den dey confab toge'rr an' mek up der min's dat 'twan't wuf de w'ile ter send anything dat walk 'pun de groun' er anything dat creep unner hit, an' dey 'gree dat dey bes' sen' sump'n dat fly 'bove hit.

"Dey study 'bout hit an' study 'bout hit, fer dey ain' wanter mek no mo' mistakes, an' las' dey mek up der min's an' dey sez ter de Hummin'-bu'd, 'Mistah Hummin'-bu'd, to be sho' you is de smalles' fowel er de lot, but you is sut'n'y de swif'es', so we gwine send you on dis errant, an' mebbe yo' size'll he'p you out ; sometimes dese yer li'l mens kin go whar de big ones kain't.'

"Hummin-bu'd he wuz a mighty peart, likely li'l feller whar ain' 'feard er nuttin' in dis 'varsil worl', an' he say, 'T'y, sut'n'y, gemmen, I be mighty glad ter go fer you-all. I'll do de bes' I kin in dis marter, dough mebbe 'twon't be much.' Den he g'long off, an' he fly an' he fly. an' he go up, up, up, ontwel he wuz higher dan de clouds. an' den he seed de Thunder an' Lightnin' keepin' gyard orer de 'bacey plant whar wuz 'way down below.

"Hummin'-bu'd he say ter hisse'f, sezee, 'Tawdy, $2 \% 1$ 


\section{A'T THE BIG IIOTSE}

lawdy! dis de time I gotter keep mighty quiet an' lay mighty low an' go mighty sof" an more mighty lively, er I be Mistah Nob'dy-f"um-no-whar befo' I knows hit.'

"He stop' whirrin' his wings an' kep' so still dat de gre`t big flamin', flarin' eyes er de Thunder an' Lightnin' ain' ketch sight ur him, an' w'en he seed a good chanct he jes' drapt right down an' sucked de flower er de 'bacey plant un' kyar'd 'way de seed in his mouf an' bringed hit ter de people.

"De Thunder an' Lightnin" rolled der eyes an' gnashed der toofs an' growled, but 'twan't no use; de people had de seed an' dey done kep' hit.

"So de Injuns owed de 'baccy ter de Hummin'-bu'd, an' dat de reason w'y. urer sence dat time dey lore de li'l Wahlelu: dat de name dey calls de pooty li'l feller whar fly so fas' he done beat de Thunder an' Lightnin'." 


\section{WHY MOLES HAVE HANDS}

One day the children came rumning to Aunt Nancy with a mole which one of the dogs had just killed. They had never seen one before and were very curious as to what it might be.

"Well, befo" de king!" said Nancy, "whar y"all bin livin' dat you nurer seed a mole befo"? "Whar you come f'um mus" be a mighty cur'ous spot ef dey ain' have no moleses dar: mus" be sump'n wrong wid dat place. I bin mos' all orer dish yer Sussex kyounty endurin' er my time, an' I ain' nuver come 'cross no place yit whar dey ain" have moleses.

"Moleses is sut'n'y cur"ous li"l creeturs," she continned. "I bin teckin' 'tickler notuss un 'em dis long time, an' dey knows mo'n you'd think fer, jes' ter look at 'em. Dough dey lives down un'need de groun', yit dey is fus'class swimmers; I done seed one, wid my own eyes, crossin' de branch, an' dey kin root 'long un'need de yearf mos" ez fas" ez a hoss kin trot on top ur hit. Y'all neenter look dat-a-way, 'kase hit's de trufe; dey's jes' built fer gittin' 'long fas' unner groun'. Der han's is bofe pickaxes an' shovels fer 'em; dey digs an' scoops wid der front ones an' kicks de dirt out de way wid der behime ones. Der strong snouts he ps 'em, too, ter push der way thu de dirt."

"Their fur is just as soft and shiny as silk," said Janey. 


\section{AT THE BIG IIOUSE}

"Yas," said Aunt Nancy, "hit's dat sof' an' shiny dat, dough dey live all time in de dirt, not a speck er dirt sticks to 'em. You ses 'sof' an' shiny ez silk,' but I tell rou hit is silk; silk clo'es, dat 'zackly w'at 'tis."

Ned laughed. "Who ever heard of an animal dressed in silk clothes?" he said.

"Nemmine," she answered, "you talks mighty peart, but I knows w'at I knows, an' dish yer I bin tellin' you is de sho'-'nuff trufe."

"Just see its paws," Janey went on, "why, they look exactly like hands."

"Look lak han's! look lak han's! umph! dey is han's, all thumbered an' fingered jes lak yo'n ; an', w'at's mo', dey wuz onct human han's; human, dey wuz so!"

"How could they erer have been human hands and then been put on a mole's body ?" asked Ned. "I believe most things you say, Aunt Nancy, but I can't swallow that."

"Dar's a li'l boy roun' dese diggin's whar talkin' mighty sassy an' rambunkshus, seem ter me. I ain' ax you ter swoller nuttin' 't all, but 'pears ter me y'all bin swollerin' dem 'ar ol' tales right an' lef', faster'n' I kin call 'em ter min', an' I ain' seed none er you choke on 'em yit, ner cry, 'nuff said. I'se 'tickler saw'y 'bout dis, 'kase I done had hit in min' ter tell you a tale 'bout huccome moleses have han'ses, whar I l'arn f'um a ooman dat come f'um Fauquier kyounty, but now dat Mars' Ned 'pear ter be so jubous 'bout hit, I ain' gwine was'e my time on folks whar ain' gwine b'lieve me, nohows. Nemmine, de chillen over on de Thompson place gwine baig me fer dat tale w'en I goes dar ag'in, an', 


\section{THY MOLES HATE HANDS}

w'at's mo', dey gwine git hit; fer dey b'lieres er'y wu'd dat draps fum my mouf, lak "twuz de law an" de gospil."

Of course, the children protested that they were as ready to hang upon her words as the Thompson children could possibly be, and presented their prior claim to the tale in such moring fashion that Aunt Xancy was finally prevailed upon to come down from her high horse and tell the story.

"I done tol' you," she said, "dat dem 'ar han's is human, an' I mean jes' w'at I ses, "kase de moleses useter be folks, sho'-'nuff folks, dough dey is all swunk up ter dis size an' der han's is all dat's lef' ter tell de tale. Yas, suh, in de ol' days, so fur back dat you kain't kyount hit, de moleses wuz folks, an' mighty proud an' biggitty folks at dat. Dey wan't gwine be ketched wearin' any er dish yer kaliker, er linsey-זroolsey, er homespun er sech ez dat, ner eren broadclawf, ner bombazine. naw suh! Dey jes tricked derse'fs out in de fines' an' shinies' er silk, nuttin' mo' ner less, an' den dey went a-traipsin' up an' down an' hether an' yon, fer tu'rr folks ter look at an' mek 'miration orer. Mo'n dat, dey 'uz so fine an' fiddlin' dey oon set foot ter de groun' lessen dar wuz a kyarpet spread down fer "em ter walk on. Dey tells me hit sut'n'y wuz a sight in de worl' ter see dem 'ar folks walkin' up an' down on de kyarpets, trailin' an' rus'lin' der silk clo'es, an' curchyin' an' bobbin' ter one nu'rr w'en dey met up, but nurer speakin' ter de common folks whar walkin' on de groun', ner eren so much ez lookin" at "em. W'ats mo", der wuz so uppish dey thought de yearf wuz too low down fer "em even ter run der eyes over, so dey went 'long wid der haids 


\section{AT TIIE BIG HOLSE}

rared an' der eyes all time lookin' up, stidder down. You kin be sho' dem gwines-on ain' mek "em pop'lous wirl tu'rr folls. 'kase people jes' natchelly kain't stan' hit ter have you thowin "up to em dat you is better'n wat dey is, w'en all de time dey knows you re nuttin' but. folks, same'z dem.

“Dey kep' gwine on so-fashion, an' gittin' mo' an' mo' pompered an' uppish, 'twel las' dey 'tracted de 'tention er de Lawd, an' He say ter Hisce'f. He do, 'Who is dese yer folks, anyhows, whar gittin' so airish, walkin' up) an' down an back an' fo th on my yearf an' spurnin' hit so 's't dey spread kyarpets 'twix' hit an' der footses, treatin' my yearf, w'at I done mek, lak 'twuz de dirt un'need der footses, an' 'spisin' der feller creeturs an' excusin' 'em er bein' common, an keepin' der eyes turnt up all de time, ez ef dey wuz too good ter look at de things I done mek an' putt on my yearf? I mus' see 'bout dis; I mus' punish dese 'sumptious people an' show 'em dat one'r my creeturs is jes' ez low down ez tu'rr, in my sight.'

"So de Lawd He pass jedgment on de moleses. Fus' He tuck an' made 'em lose der human shape an' den He swunk 'em up ontwel dey 'z no bigger'n dey is now, dat 'uz ter show'em how no-kyount dey wuz in His sight. Den bekase dey thought derse'fs too good ter walk 'pun de bare groun' He sont 'em ter live unneed hit, whar dey hatter dig an' scratch der way 'long. Las' ur all He tuck an' tuck 'way der eyes an' made 'em blin', dat's 'kase dey done spise ter look at der feller creeturs. But He feel kind er saw'y fer 'em w'en He git dat fur. an' $\mathrm{He}$ ain' wanter punish 'em too hairy, so He lef' 'em dese silk clo'es whar I done tol' you 'bout, an' dese han's whar 


\section{THY MOLES HATE HANDS}

you kin see fer yo ‘se'fs is human. an’ I reckon bofe dem things putt 'em in min' er w'at dẹ useter be an' mek 'em 'umble. Trer sence den de moleses bin gwine 'long un'need de groun'. 'cordin ter de jedgmen' er de Lawd, an diggin' an' scratchin' der way thu de worl, in trial an' tribilashun, wid dem po' li'l human han'ses. In' dat orter l'arn you w'at comes er folks 'spisin' der feller creeturs, an' I want y'all ter 'member dat nex' time I year you call dem Thompson chillen 'trash.' "

"I'd like to know what use moles are," said Ned, who was of rather an investigating turn of mind; "they just go around rooting through the ground spoiling people's gardens, and I don't see what theyre good for; you can't eat them or use them any way."

"Sho', chil"." said Aunt Nancy, "you dunno w'at you talkin' 'bout; de Lawd hare some use fer ery creetur He done mek. Dey tells me dat de moleses eats up lots er bugs an' w'ms an' sech ez dat. dat mought hurt de craps ef dey wuz let ter live. Sidesen dat, jes' gimme one'r de claws er dat mole, an' lemme hang hit roun' de neck ur a baby whar cuttin' his toofs, an' I boun' you, ery toof in his jaws gwine come bustin thu his goms widout nair' a ache er a pain ter let him know dey's dar. Don' talk ter me 'bout de moleses bein' wufless! I done walk de flo' too much wid cryin' babies not ter know de use er moleses."

"You don t really believe that, do you?" asked Ned.

"B'lieve hit!" sne answered indignantly: "I don' b'lieve hit, I knous hit. I done tol' you all de things a hyar's foot kin do: wats de reason a mole's foot ain' good fer sump'n, too? Ef folks on 'y knowed mo' about sech kyores ez dat dar neenter be so much sickness an' 


\section{AT THE BIG IIOCSE}

mis'ry in de worl'. I done kyored myse'f er de rheumatiz in my right arm jes' by tyin' a eel-skin roun' hit, an' ev'ybily on dis plantation knows dat ef you'll wrop a chil's hya'r wid eel-skin strings hit's boun' ter mek hit grow. Ef you want de chil' hisse'f ter grow an' ter walk soon you mus' bresh his feet wid de broom. I oon tell you dis ef I hadn' tried 'em myse'f. You mus'n' talk so biggitty 'bout w'at you dumno nuttin' 't all about. You come f'um up Norf yonner, an' mebbe dese things don' w'u'k de same dar ez w'at dey does down yer on dis ol' plantation whar we bin 'pendin' on 'em so long." 


\section{THE FOX ANI) THE DLCK}

One night when Aunt Nancy was putting the children to bed, and they were, as usual, begging for a story to go to sleep upon, she asked, "Is I uver tol" you anything" "bout ol Mis" Inuck?".

The children said that she had never told them a duck story, but that Aunt "Phrony had.

"Lawsy! I dumno huccome I ter fergit ter tell you 'bout her," she said; "mobbe 'kase she sech a li"l runt ur a fowl. But nemmine dat, she got plenty sense, an' one time she fool Mistah Fox, sho' 'nuff. Look lak some pusson er ru'rr all time foolin' him. an I kain't 'zackly see thu dat, 'Kase dese days he's mighty smart an' up ter snuff an' kin dodge de houn's an' de hunters de pooties' urer you see. Looks lak in dem days things wuz sort er lak dreams, jes' de contrary-ways f"um w'at dey is now. Anyways, she knowed how ter bamboozle him somehow er ru'rr.

"One time she wuz gwine waddlin" lown ter de branch, talkin' 'way ter huseef, hard 'z she kin, rumnin' on quackin' an' scoldin' 'bout dis an' dat. Mistah Fox he wuz nosin' roun' thu de woods an' he year her, an' he say ter hisce'f. he do, 'Ter's whar I gwine git me a good meal er vittles: I reckon she jes' bout big 'nuff ter fill me chock-full, an' she look right roung, so I 'spec' she's 


\section{AT TILE BIG HOLSE}

plump an' tender.' Ife smack his lips an' go rackin' romı ter de front an' squat down behime a tree, an' w'en she git mos' up ter him he jump out an' nab her

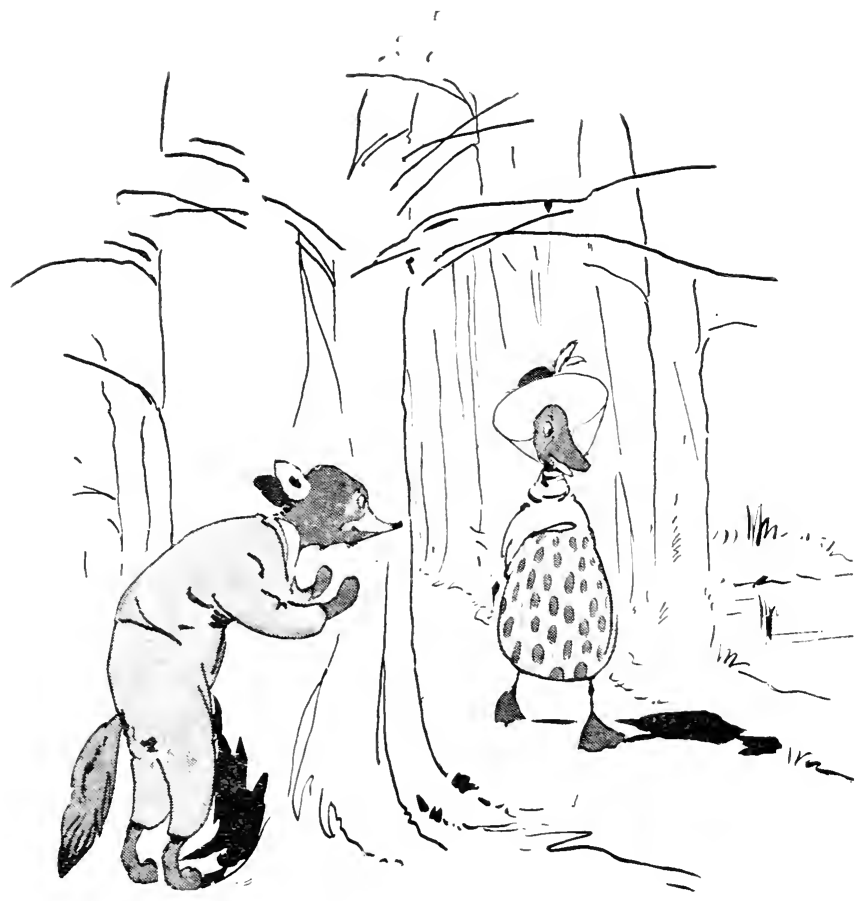

by de neck an' fling 'er 'cross his shoulder an' g'long singin'.

"All de time he wuz singin' Mis' Duck she wuz hangin' haid down, jes' a-kickin' an' a-scufflin', but 'twan't no use; Fox he hilt on. he did, an' he jes' let her go on jawin' all she wanter. Las' she stop her racket an' set 280 


\section{THE FOX AND THE DUCK}

her min' ter wu'k on de queschin uv how ter git outer de pickle whar she wuz in. Pres'n'y she say, 'Scuse me, please, suh, Mistah Slickry Sly-fox, I lak ter talk to you a li'l bit, but hit's mighty hard wu'k wid my haid hangin' down so turr"bl slanchindicular. I ax you, please fer ter fix me wid my haid a li'l higher, suh.'

" 'Naw, ma'am', sez de Fox, sezee, 'I knows 'nuff ter hol' onter a good thing w'en I gits hit.'

"'Laws-a-mussy, Mistah Fox,' sez Duck, sez she, 'you is mighty flattersome in yo" remarks; I ain' gwine try ter chouse you outer yo' dinner, 'deed I ain'. But w'ile we joggin "long yer, I jes" lak ter ax you, suh, ef you lak pig ter eat?'

" 'Now you talkin',' sezee, an' he smack his lips so loud dat hit sont col' shivers up an' down Mis' Duck's spine, an' he say, 'T'at de marter, Mis' Duck; w'at you shiver 'bout dis wo'm day?'

"She wuz too proud ter let on dat she wuz 'feard, so she say, 'Oh, nuttin' 't all, Mistah Fox, jes' a li'l tetch er de ager; you know w'en you got dat you 'bleeged ter shirer, nemmine how wo'm de day is.' Den she go on ag'in 'bout pig. She say, 'So you sho' 'nuff does lak pig, suh. I done year tell you did, but I wan 't sho" 'bout hit.'

"Fox he got his min' on pig an' seem lak he kain't git hit off, an' las' he git kind er mad kase dar wan't no pig ter be had. an' he say, sezee, 'Look-a-yer, ooman. I dunno huccome you name dat meat ter me w'en you knows I kain't git me none.'

"Mis' Duck she ses, sez she, 'Now, I ax you fer ter tell me de plain trufe. suh; w'icht does you perfer, pig er duck?"

“Sezee, 'Gimme pig ev'y time, 'kase 'tain' got no 281 


\section{AT THE BIG HOTSE}

fedders. I tell rou, ma'am, w'en rou swollers duck down de way I does, fedders an" all. yo" thoat gits mighty woolly sometimes. I e'en about 'spec' I'll choke ter def rit on duck some er dese times.'

"Sarre you right. an' I hopes you will,' sez Mis' Duck ter husse'f. Den she sing out. "So you perfers pig. suh. Putt me down, den, land knows! an' I'll show you whar you kin git you a hull litter er pigs!"

“Fox sezee, 'Tellum. yer goes fer de pigs, an' min' you walk straight, fer ef I fin' any foolin' 'bout dis bizness I ain gwine gin rou time ter say yo' pra'rs. You jes" moser 'long in front er me, please ma'am.'

"She tuck an" tuck him to a place whar a gus"-root wuz blowed up clean outen de grom", an" she p"inted hit out to 'im an' tol' 'im dat behime dar wuz de litter er pigs, an' dat he mus' run f'um a piece off an' jump de gus'-root right inter de baid er pigs an' s'prise 'em data-way, so's't he cu'd git 'em easy.

"Ol' Fox he 'gree ter dis, an' he run back a li'l ways an' tuck a big bref an' spit on his han's an' th'owed one foot front an' tu'rr one behime, an' tectered back an' fo th a minnit ter git a good swing on him. Den he bus' inter a run an' lipt de gus'-root widout techin' foot to hit an' lan'er in de midse er de litter. But bless goodress, 'twan't no litter er pigs, 'twuz ol' Mis' Dog an' her li'l ehillen.

"'Ow! wow!' sez she. 'who dis owdacious ereetur dat come a-r'arin' an' a-chargin' inter my house, 'sturbin me an' my fambly? Ef 'tain' dat good-fer-nuttin' piece ur imp ence. ol' man Fox. I gwine show you huccome, dat I is ! I let you know you kain 't come 'strovin' de peace uv'spectable folks no sech a ways ez dat. Stay yer one 


\section{THE FOI AND THE DCOK}

minnit longer an' I gwine putt my font down yo' thoat!"

"Wid dat Fox he lipt outer de baid faster dan he got in an jes" went a-sootin", Mis" Dog atter him. hot-foot. Mis' Duck she wuz behine de gusoot jes' a-snickerin" an' a-snortin'. Fox he ketched sight ur her an' he sung out, "Xemmine. Mis" Duck. you is de "casion ur all dis rumpus. I reckon you think dish yer's a mighty funny prank you played me, but I gwine pay you back fer dis: jes" you wait. ma am. Sou grwine teck nu'rr free ride on my shoulder 'fo' you knows hit. an' lat time you won't git off so easy. Iou kin pin hit rown in ro "memb ance dat I gwine pay you hack wid int res' on de money.'

"Mis" Duck she knowed he dasent ter stop right den, so she let out one big hoot an" she ses. sez she. "Fo do, Mistah Fox, so do. Now's yo' time, suh; de sooner de better. Whrn't you stop. suh? Sou ain nuver gwine ketch me no younger. Better not wait, suh; de longer you waits de older an' tougher I grows.'

"But ol man Fox knowed too much ter stop w'en Mis", Dog wuz at his heels. so he went a-sailin' an lef" Mis' Duck stannin' by de puppies jes' a-quackin' an' a-cacklin' an' a-laughin'. An' urer sence dat time dar ain' bin much love los' 'twix de foxes an' de ducks. I reckon de foxes has done paid 'em out mo'n a hunderd times fer dat trick." 


\section{WHY THE GROUND-HOG HAS A SHORT TAIL}

One erening the children went down to Aunt 'Phro$n y$ 's cabin and found her and her grandson. William, hovering over a handful of embers, for the nights were growing a little chilly. The old woman was singing, and she rocked back and forth in time to the music, as she led with the words of the hymm. while William joined in the refrain of the old-time favorite,

\section{DOWX BY THE RIVER}

Whar's all dem chillen dress' in white,

Dou'n by de river?

IIus' be de chillen er de Is'elite,

Doun by de river side.

Refrain: Oh! well end dis war,

Dou'n by the river;

We'll end dis urar,

Down by de river side.

Whar's all dem chillen dress' in red,

Doun by de river?

Mus' be de chillen whar Moses Ted.

Doun by de river side.-Refrain. 


\section{WHY THE GROLND-HOG HAS A SHORT TAL}

IThar's all dem chillen dress' in green,

Doun by de river?

IIus' be de chitlen whar Jostua seen,

Doun by de river side.-Refrain.

Whar's all dem chillen dress' in pink,

Doun by de river?

Mus' be de chitlen whar teckin' a drink,

Dou'n by de river side.-Refrain.

Whar's all dem chillen dress' in yaller,

Doun by de river?

Oh, dar whar de water it run mighty shaller,

Dorn by de river side.-Refrain.

Thar's all dem chillen dress' in gray,

Dou'n by de river?

Ins' be de chillen whar squandered away,

Dou'n by de river side.-Refrain.

Thar's all dem chillen dress' in black,

Dou'n by de river?

IIus' be de chitlen whar done turn back,

Dou'n by de river side.-Refrain.

Whar's all dem chillen dress' in blue,

Doun by de river?

Mus' be de chillen whar done come thu,

Doun by de river side.-Refrain.

The children waited until the Israelites were safely brought through before announcing themselves, when 285 


\section{AT TIIE BIG IIOTSE}

they were cordially welenmed to the fireside, for Aunt 'Phrony was in an unusually genial mood. possibly induced by her farorite hymn and the trimphant passage of the children of Israel.

"Come right in yer an' set yo" li"l se'fs down by de fire," she said. "snip, git yo" lazy kyarkiss offen dat cheer an' let Niss Janey have hit. Min' w'at I say to rou. cat. er I come dar an' cuff you. Snap, teck yo'se'f offen dat h'ath an' gin dese chillen room ter putt der footses down. I nuver seed a dog dat cu'd spread hisse'f orer so much ha'th-room ez w'at he kin. Well, chillen, I bin studyin' 'bout y'all dis re'y day. Wi'yum he bin huntin' an bringed home a groun '-hog wid him an' dat putt me in min' ur a tale I wanter tell y'all 'bout, how de groun'-hog come ter have such a shawt tail.

"One time he wuz gine "long studyin' 'bout odds an' een's an' not payin no 'tention ter whar he wuz er w'at he wuz doin', an' fus' news he knowed he run spang up ag'in a gang er wolfs an' dey nabbed him in a jiff an' wuz g'winter mek a meal offen him, den an' dar. Groun'-hog he wuz right boddered. 'How in de name er common sense did I run my haid inter sech a trap ez dis?' sezee; 'look lak I bin rootin' roun' thu de worl' 'long 'nuff ter keep onten sech messes ez dis. Well, I reckon nob'dy kin live long 'nuff not ter gin hisse'f a s'prise now an' den. De thing fer me is not ter let on befo' dese gemmen dat I'se skeered, an' mebbe I'll pull thu yit somehow er ru'rr.'

"But w'en dey 'menced rampin' an' roarin' all roun' 'im an' gnashin' der toofs at 'im hit mek 'im feel kind er faintyfied. an' he say ter hisse'f. he do, 'Lawd, lawd! I wish I bin sassified ter stay home un'need de groun' 
dis day!" ITe ain" let on, dough, an' pres'n'y he mek out ter say, "Gemmen-for I kin see you is sho"'nuff gemmen by de lookin: ur you-gemmen, I knows yall done bin roun' a heap an'seed a lot. but I boun' you dar's one thing you ain' seed in all yo bawn days.'

"De wolfs jes" howled an" langhed wid scawn ter think dar wuz anything lef" dat dey ain' seed. 'T"ats dat?' dey ses; "name dat to us ef you please. Sump'n dat weall ain' seed, much e' we hin romantin' up an' down de worl"! We lak tor know mighty well w'at dat kin be.'

" "Well. now I tell you,' sez de Groun'-hog, sezee, 'I be willin' ter bet dese yer two years offen my haid dat wid all yo" goin" up an' down an' back an' fo th an' to an' fro, none er you has uver seed a grom'-hog darnse; is you now?"

"De wolfs dey hunched up der shoulders at one nu'rr an' dey ses, 'Shucks! dish yer plumb reedikelous, de idee ur a groun'-hog darnsin'; who urer yearn er de likes er dat?'

“'Temmine,' sez de Groun'-hog, 'ef y’all doan b’lieve me I kin show you; "twon't teck but a minnit; I'll bofe sing an' darnse fer you, an' I ain gwine tas'e any de worse fer dat, I reckon. I boun' you. I kin gin y'all some 'musement an' rit eat jes' ez good den ez now. 'Twon't teck long, nohows.'

"De wolfs dey insulted toge'rr an' greed ter let him try. 'Twon't mount ter' shucks, nolow;' dey ses; 'mought ez well let him have one li'l whu'l befo' we swaller him.'

"Groun'-hog he ses, 'Gemmen, I gwine play you fa'r, an' ter mek sho" dat I don" git "way f"um you. you kin mek a succle er yo'se'fs an' s'roun' me on all sides.' 


\section{AT THE BIG HOLSE}

"So de wolfs dey do dat; dey mek a succle all roun' him an gin him a big range, an' squatted dar on der ha'nches wid der big red tongues lollin' outer der moufs an' der long toofs a-showin', glarin' at him wid der ol' green eves. Groun'-hog he 'mence ter sing lak dis:

$$
\begin{aligned}
& \text { 'Di-a-di-a-hey, di-a-di-a-hey, } \\
& \text { Di-a-di-a, di-a-di-a, di-a-di-a-hey.' }
\end{aligned}
$$

"At de same time he darnse, an' ur all de capers you urer seed, dem tuck de lead, fer ol' man Groun'-hog wuz darnsin' fer his life, he wuz. He kep' flippin' his pars in time ter de music, an' he wagged dem behime footses so fas' dat hit look lak he jes' hangin' in de air widout no laigs twix' him an' de groun'. All ter onct he'd spin clean roun' on one foot an' den go on ag'in, faster dan befo'. De wolfs dey wuz might'ly 'mused, an' dey jes' clapped der han's an' hollered an' mos' fergit dey wuz gwine eat him, but I boun' you Groun'-hog he ain' fergit hit.

"Las' he gin one gre't han'-spring backwu'ds an' disappeart lak he wnz wipe offen de face er de yearf, an' lef' de wolfs settin' dar on der ha'nches. Dey look behime an' dey look befo' an' dey look on de groun' an' den up in de air, ez ef dey thought mebbe he done jump so high he ain' come down yit; but dey ain' see Mistah Groun'-hog.

" 'Now. whar you s'pose dat triflin creetur gone to?' dey ses ter one nu'rr. Las' one ur em' whar wuz settin' up hol'in' a piece ur a tail in his han' he say, he do, 'Umph! I don' s'pose, I knous. Dat sut'n'y is a slick li'l creetur. Te-all ain' notuss dat de place he pick out 


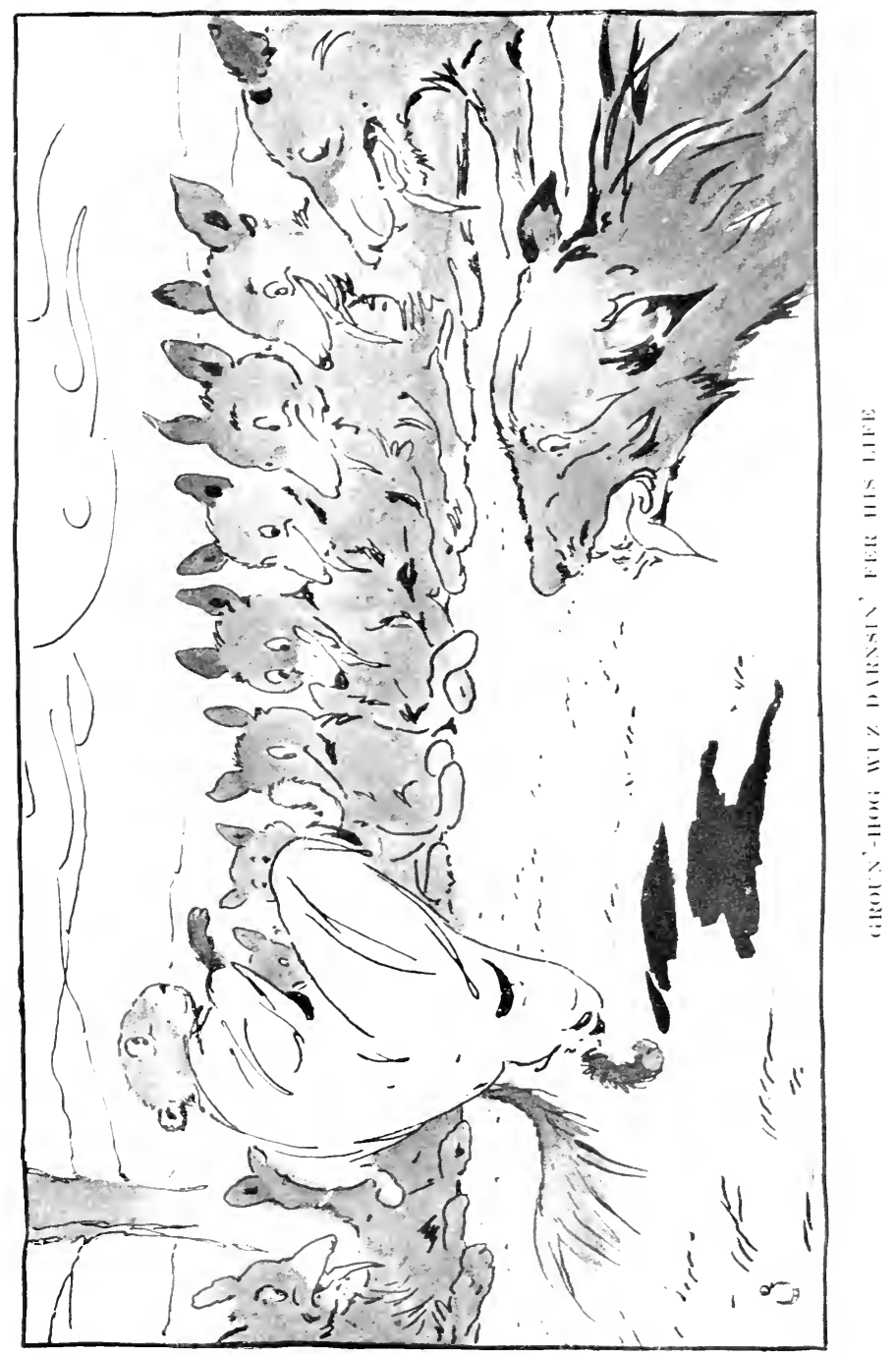





\section{WHY THE GROUND-HOG HAS A SIIORT TAIL}

fer ter darnse in wuz mighty nigh ter de hole whar he lire. He kep' a-airlgin' an' a-aidgin nearer an' nearer, an' las' he gin one spring, an' down he went. I wuz de neares" $1 \mathrm{r}$ any an' I seed him goin'. I grabbed him by de tail, but suz! he wuz gwine so fas' dat nuttin' cu'd stop him, an' de tail jes' natchelly bruk off an' lef' dish yer piece in my han'?

" "Tell, of dat don' beat bowser!" sez de wolfs, an' den dey laugh ter think how dat li'l creetur done fool 'em, ontwel one un 'em clap his han' on his stummick an' 'low he feel mighty empty, an' den dey all laughed on de wrong side er der moufs. kase dey knowed dey gotter do widout dimner dat day.

"Now dat"s de reason de groun'-hogs got sech shawt tails, an' Wi'yum kin go an' git dat one whar he ketched an' show you de trufe er w'at I bin tellin' you. Iou year me. Wi'rum! Don' you sot dar lookin' at me, suh! You mosey 'long out ter de shed an' git dat groun'-hog dis re y minnit, lessen you wants me ter come dar an" walk ip an' down yo' body a time er two! Wi'yum do lak he wuz hung on henges an' de henges wuz all rusted out, but I boun' you ef I wuz ter say 'supper' ter Wi'yum. he'd move right lively." 


\section{MR. HARE, MIR. WILDCAT AND MR. OTTER}

When the ground-hog had been duly examined and commented on the children hinted that they were ready for another story. Aunt 'Phrony at first refused, saying that it was time they were home tucked up in bed, ready for the nightly risit of the Sand Man. When they insisted that they were nerer wider awake in their lives, she said, "Well, mebbe you inn" sleepr, but mebbe I is. High time I knocked de ashes outen dis pipe an" hanged my ol' bones up on de baid." But finally she gave in to the combined persuasions of the three and consented to tell the story of the hare, the wild-cat and the otter.

"One time," she began. "ol' Hrar" he wuz squanderin' roun' thu de woods wid a pipe stuck in his mouf, idlin' "way de time, w'en all ter onct Mistah Wil'cat jump out

- f'um behime a big tree an' yell, 'Boo!' at him an' fetch a grab dat all but ketch him. Hyar' wuz sho'-nuff s'prised dat time, but he gin a big jump ter one side an' lan'ed behime a tree an' stood dar ez still ez a mouse, sca'cely drorin' his bref in an' out, not dastin' even ter peek so"ster see of Wil'cat wuz still dar. He got mighty tired an 'cramp' up befo' long, an' sezee ter hissef, 'MLussy me! w'at oon I gin jes' ter wall my eye roun' dis tree an' see w'at dat mis'able creetur doin'. I ain' year nair' soun': melbe he gone on. Ef he's tired ez w'at I is, I 'spec' he tone gin de marter up an' moseyed 'long ter git his rittles som'ers else.' 
Wid dat he -tick lis nose ont ter see wat gwine on. an' dat wuz 'nulf fer'ol' Wil'cat: he jes' gin one monst'ous jump an" lan 'ed right onter de II rar", an' den dar wan't no sech thing ez gittin "way. I tell you. No use ter kick an' squall: dem claws jes" curled derse fs right

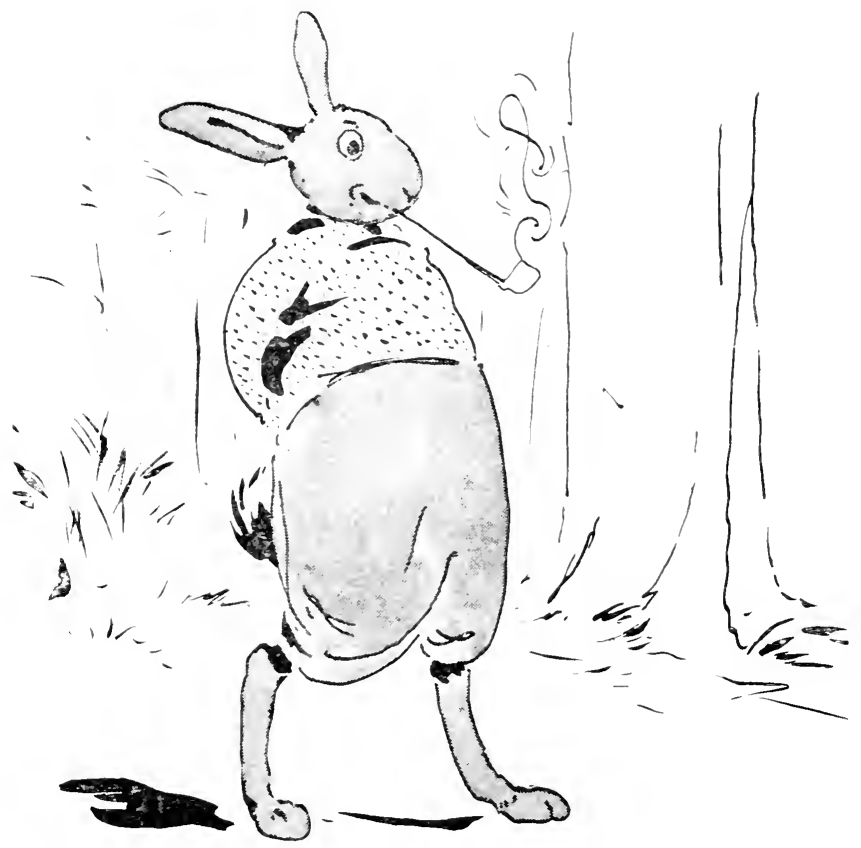

inter him an' stuck lar'. much ez ter say, 'Yer we is, suh, now lessee you onloose yo'se'f.'

"Tril'cat hol' him up' in front ur him an' talk 'way. at him lak Hyar' wuz de pris'ner at de bar an' he wuz de jedge an' de jury an' de hull co'te th'owed in. an 


\section{AT THE BIG HOLSE}

ev'y now an' den he gin him a shake dat mek er'y toof in his haid rattle lak de seed in a dry go de. 'Lh-huh!' he say, sezee, 'I bin wantin' ter git my claws inter you dis long time! Meddle in urr folkses bizness, will you? Go roun' an' hunt up mischief an' play tricks on yo' betters, will you? You got a lot er gumption, I reckon ; dey tells me you has, anyways; but dat ain' gwine do a man much good w'en his time comes; no pusson so smart dat he kin keep outen de sclutches ur ol' man Def, an' dat's right whar you is dis ve y minnit, I tells you dat p'in'-blank, an' you'll know I bin tellin' you de trufe w'en I 'mence ter mek minch-meat uv you, w'ich is gwine be did dis ve'y minnit, ef not sooner.'

"Hyar' he feel kind er pa"lyze w'en he year dat sort er talk, but pres'n'y he pick hisse'f toge'rr, an' sezee, wid a mighty pleasin' smile, 'Mistah Wil'cat, dish yer yo' imnin's, an' I reckon you gwine do w'at you please wid me; I ain' gwine 'spute dat, but I ax you dis, suh, is I a fitten dimner fer sech as you? Ain' I lean ez a razorback hog? Is I monn one good moufful, an' sca'cely dat? You kain't 'ny dat, suh. Now I ax you ter 'member de time w'en I done fill you up chock-full er tukkey, widout you havin' no mo' ter do dan lay still an' let on you'se 'sleep w'iles I toll de tukkies up to you wid singin' an' darnsin'. I kin do dat ag'in, an' better'n dat. Jes' name yo game, an 'I'se de man whar kin git hit fer you. Don be too modes", suh; big game er li'l game, hit's all de same ter me.

"Wil'cat he study for a minnit an' den he say, 'Well, I'd lak mighty well ter git me a tas'e er deer meat onct mo'. So long sence I set my toofs inter a piece dat I 'clar' I mos' fergit how hit do tas'e. I bin livin' on shawt 


\section{MR. HARE, MIR. IVILDCAT AND MIR. OTTER}

commons dis long time, an' I'se dat hongry I'se nigh stracted. Ef you kin git me some deer I gwine let you off dis time: but min' you. Mistah Hyar', no tricks 'bout dis er I frail you so hard you'll wish Iid et you up 'stidder clawin' you inter grape-vine strings. Tou know me, sulu, an' you knows I don' stan' no triflin'.'

"Dat's all right, suh, dat's all right,' sez Hyar', sezee, 'jes' you come 'long er me an' I show you dat I'se a man er my wid. We gotter go down ter de place whar de deers come ter drink an tur eat de moss, an' dar I gwine show you wat I kin do, ef $I$ is no mo'n a li'l shawt-tail runt: fer dat: wat I year some er de folks bin callin' me. Step 'long dis way, suh, ef rou please.'

"Dey went on down ter de branch whar de deers come ter drink, an' Hyar' he say. 'Now, Mistah Wil'cat, jes' you set dar in de bushes an' hide, an' I'll crope out on dat limb whar hang over de water, an' w'en de deer comes I'll jes' drap spang onter his back, an' w'en he 'mences ter r'ar an' splunge an' mek fer de bank, den's de time you mus' jump out an' nab him.' All de time he say ter hisse'f, 'Tas, lawd! an' w'en I onct git onter dat deer's back 'tain' gwine teck me long ter jump ter tu'rr bank an' git outer yo' way, suh!'

"Wil'cat he say, 'All right, suh, go ahaid. But min' you. fa'r play, now. None er yo' tricks an' traps wid me, er I gwine come a-rattlin' an a-shattlin' down dar an' jes' natchelly scrape you inter fiddle-strings.'

"So Wil"cat he squat down in de bushes nigh de bank an' Hyar' he crope ont on de limb, an' dẹ waited an' dey waited 'twel Wil'cat wuz fair' frazzled. Tas'. yer come a deer lopin' down ter de water. an' he waded right in an' drunk his fill an' den stond dar dippin' his haid 


\section{AT TIIE BIG IIOLSE}

in clean up ter de eres, lookin' fer moss ter eat. Hyar' he "low ter hisse'f, 'Lm-humph! yer whar I gwine roust him outer dat in a hurry. Won' he wunner w'ats got him all ur a suddint?'

"He tucken de time w"en de deer's haid wuz un'need de water ter drap smack onter his back. Co'se de deer "menced ter ra'r an splunge an Hyar" he scrabbled an' sclutched ter hol' on long 'nuff ter jump ter tu rr bank, but lawsy! ol' man Deer jes" natchelly shuk him off inter de water an' den jumped fer tu'rr bank an' made off inter de woods.

"Hyar" he ses ter hisse'f, 'Now, did any pusson urer see de beat er dat onfren"ly Deer: "gredge me a li'l foothol' on his back! Ef I wuz ez big ez wat he is I sut'n'y oon be ez mean an' stingy wid my back ez dat.'

"He 'gun ter sink 'bout den. an' he see dar wan't nuttin' fer hit but ter git drownded, so he sing out ter de Wil'cat fer he'p, but shucks! no cat s gwine git. hits paws wet ef hit kin he"p. so he jes' turnt his back an' went off spittin' an' snarlin, 'kase he done los' his dimner.

"'Bout den 'long come a Otter down ter de bank, an' Hyar' he call out fer he'p. He say, sezee, 'Oh, Mistah Otter, he'p me outer dish yer pickle an' I gin you anything you want:; 'deed I will!'

" "W'at you gimme?" sez de Otter, sezee.

"Sho"! sez ol' Hyar', spittin' and sputterin' an' chokin', 'has I gotter drown wile I mek promusses? Git me outen dis an den I mek any promuss you ax me to. Ef you gwine he'p me. man. he p me dis re'y minnit er hit be too late in de day, an' my def gwine be on yo' haid.' 


\section{MIR. HARE, MIR. IIILDCAT AND MR. OTTER}

"No Otter he swim in, 'kase he wuz a fus'-class swimmer, he wuz, an' fished ol' man Ityar' out an' set him on de bank at de aidge er de water. Dar he sot all weak an' trim'lin', no bigger 'n a han'ful, but he wan't so fur gone but w'at dar wuz plenty. er 'havishness lef' in him yit, an' he say ter Otter, sezee, 'Please, suh, ter lemme rum up de bank an' shake myse'f, so's'ter git some er de water outen my fur; 'pears lak I done soaked up de hull branch an' bringed hit out wid me.'

" 'W'y sut'n'y' sez de Otter, sezee, 'I knows you is a lan' man; an' co'se you ain' useter bein' in de water, co'se you don' fin' hit 'greeable. Jes' g'long an' shake hit outen you, an' I'll wait fer you yer on de bank.'

"Wid dat Hyar" went kitin' up de bank an' gin hisseff a good shake an' den lipt inter de bushes an' wuz off lak a house afire. 'Tait fer me on de bank, suh!' he sung out, an' he say ter hisseff, 'Yas, suh, I 'spec' you wait fer me on dat bank one w'ile 'fo' you see me ag'in.'

" 'Umph!" sez Otter ter hisse'f, 'umph! promuss me anything I want an' den not so much ez stop ter mek his manners! 'Pears lak 'twan't even a thanky job,' an' wid dat he went on 'bout his w'k in de branch, 'kase he wuz mighty busy ketchin' fish fer his fambly, fer he wuz a gre te fisherman an' a pow'ful swif" swimmer an' cu'd jes' rum a fish down in no time 't all. He ketched him two fish at onct an' tuck 'em in his mouf an' went inter his house, w ich 'twuz a deep toole in de bank. But dough he kep' on 'bout his bizness, he ain' fergit dat trick uv ol' Hyar's, an' no mo' did Mistah Wil'eat, an' w'en dey tell tu'rr creeturs 'bout him dẹ all git mo' riled up 'gins' him dan befo', an' dey ses. 'Now ain' dat de wus' trick vit? Done fool two spectable gemmen lak Mistah 29.) 


\section{AT TIIE BIG HOUSE}

Wril'eat an' Mistah Otter at one an' de same time. Done prove hisse'f one time mo' cz slipp'y an' onreliable ez a eel; mo` chanct er missin' him dan er gittin' him. Temmine, ef we jes' wait long 'nuff de time boun' ter roll roun' w'en we gwine git de unner-holt, an' den we'll jes" natchelly wipe up de rearf wid him befo' we wipes him clean offen de face er creashum.'

"An' now," concluded Aunt 'Phrony, "y'all chillen neenter pester me no mo' dis night, 'kase my ol' breens is so wo' out wid all dish yer tryin' ter 'member, dat ef you wuz ter crowd me any mo' right now dey mought crack an' let all de tales run out. Wi'yum, quit layin' ro`se"f all orer dat flo", same ez a spraddle-bug, an' go an' git de lantu'n an' light dese chillen up ter de house. You year me, suh!"’ 


\section{MR. HARE AND MR. ELEPHANT}

One day Aunt Nancy was making little Kit's toilet, trying her best to wash his neck and ears while he fidgeted and dodged and stood first on one foot and then on the other, and declared, after the manner of small boys, that he was not dirty, and if he was, he preferred being so.

"Tell, ef urer I spected ter year a chil" er my young Miss' talkin' dat scannelous!" she exclaimed. "Anyb'dy mought think "twuz one $\mathrm{r}$ dem Thompson chillen, on'y I nuver knowed "em ter talk so much lak po" whitetrash. Who urer year er de qual'ty gwine roun wid dirty years? Hit 'min's me er de time w'en Mistah Efalent oon wash his years. Sho! you kin stan" still ter lissen at tales, kin you? Well, now, you jes' hol' right still, lak a good li'l boy, an' lemme git inside dis urr year, an' I tell you de tale w'iles I go 'long."

Here she commenced a not too gentle attack upon the other ear, holding him firmly by the burning member already cleansed, as she told the story of the careless Mr. Elephant, while Janey and Ned listened with a less chastened enjoyment than that of the little boy who was haring his ears scrubbed.

"One time," she said, "dar wuz gwine be a big meetin" 'mongs' de creeturs, an' Mistah Efalent he so anxious ter git dar on time dat he plumb fergit ter wash his years. 


\section{AT TIIE BIG IIOCSE}

Mis' Efalent she wuz a mighty p'tickler ol' lady, an' she eall out atter him an' tell him 'bout hit. She say: 'Gracious ter gondness! Mistah Efalent, 'tain poss'ble you gwine 'mongs' all dem pryin', peerin' creeturs wid dem dirty years? Fer de sake er ro" fambly, come back yer an' fix yo'se'f nice an' proper.' Efalent he let on he ain' year her, jes' lak mens does sometimes w'en der wifes is talkin' at 'em, an" 'twan't mo'n th' ee winks befo' he wuz outer sight an' rearin'.

"Bout dat same time Hyar" he wuz primpin' hisse'f up fer de meetin', "kase he wuz mighty kyareful bout fixin' hisse 'f up nice an clean. He washed his face wid his paw an' licked his fur down an' den got ol' Mis' Molly Hyar' ter wash his years husse'f, an' all roun' behime 'em, so'st de folks whar set behime him in de meetin' kain't excuse him er not bein' neat. Den he had some notion er lettin' on dat he wuz single an' flyin' roun' 'mongs' de gals a li'l, so co'se he wanter look mighty spruce. Las' he start off an' git down ter de rirer 'bout de time Mistah Efalent git dar.

"He bin wumnerin' ter hisse. $\mathrm{f}$ how in de name er goodness be gwine git over de river, an' w'en he see de Efalent he say ter hisse'f. he do: 'I sut'n'y is de gre'tes' man fer luck dat uver hopped on fo' laigs. Dish yer whar I gwine git 'cross widout even so much ez gittin' a paw wet.' Wid dat he walked up ter de Efalent an' 'menced muchin' him. 'Howdy, Mistah Efalent,' sezee, 'I hope I fin's you well an' in de enj'yment er good healt'. You so gre't an' gran', suh, dat I reckon w'en you does git a mis 'ry hit boun' ter be a pow'ful big one.'

"W'at's dis a-talkin" at me?' sez de Efalent, sezee, an' he look all roun' fur an' near an' las' he seed 298 


\section{IIR. HARE AND MR. ELEPHANT}

Hyar' settin' down mos' unner his front footses an' cuttin' one ere up at him, sass ez you please. 'Fo' gracious!" sezee. "look out dar'! li'l mo'n I'd a-tromped on you, sho"! Who is you. anyhows"'

"Den Hyar" he 'mence ter "splain hisse"f an' tell whar he wuz grine an' baig Mistah Efalent ter tote him cross de river. Hit sut'n'y wuz a sight ter mek you laugh, des tells me, ter see dat li'l feller settin' up dar on his ha'nches. haid thowed back so"t he cu'd look Efalent in de eve. an' tongue goin' same`z a mill-clapper. Ol' Efalent stood dar squintin' down at him an' lis'nin' at him, w'ile bofe gre 't big rears went flippittrflap, flippitty-flap, an' his trunk wuz jes a-marin' an' a-rearin' an" a-curlin" rom" thu de air.

"Las' Hyar" he stop 'long 'nuff ter git bref, an' Efalent he say, sezee, "Shucks! man. I kain't stop to tote you orer de river; I must be at de meetin' on time.'

"Lawsy!" Hyar" 'low, "tain' gwine teck you long ter tote a li'l feller lak me.'

"Dat mek Mistah Efalent laugh an' he say, sezee, 'How sech a li'l feller ez you gwine stick on my back?' "Oh. nemmine, sezee: 'I tell you de way we kin fix hit. Jes" you teck an" putt me inside er one "r yo" years an' let de flap down orer me ter keep me in, an' I boun' you. I go orer safe an" soun'?

".All right." sez de Efalent. sezee, an' he tuck an' tuck his trunk an wrop hit roun' Hyar' an' gin him a whu'l thu de air dat tucken his bref clean 'way, an' he lan'ed him in de rear an' shet de flap down on him. Den he splunge inter de river an' 'mence ter cross orer. "Co"se Hyar" ain' bin in de year long 'trol he 'gun ter study w'at mischief ter git inter. He 'mence ter 


\section{AT THE BIG HOUSE}

fidget roun' an' wu'k his nose hard an' sniff lak he smell sump'n pow'ful bad.

" "Wa't"s de marter wid you?' sez de Efalent, sezee.

"Hyar" he 'spon', 'Oh, nuttin' 't all, Mistah Efalent; don' you min' me, suh ; jes' go right 'long.'

"Pres'n'y he go at hit ag'in, jes' sniffin' an' kyar'yin' on. 'Tm-m-m-umph! umph! umph!! umph!!!' sezee, hol'in' his nose lak he kain't stan' ter keep hit open.

"'Gre"t king!" sez de Efalent, 'w'at is de marter wid de li'l cuss, anyhows?'

“'Don' pay no 'tention ter me, suh,' Hyar' say. 'I has de as'my an' dat mek me breave hard; soun' mighty loud, 'kase I'se right inside yo' year.'

"Well, suh, he kep' hit up dat-a-way on de trip over, an' w'en dey git ter tu'rr side he watch his chanct w'en Mistah Efalent flip his year up an' den he jes' lit down same'z he bin made er injy-rubber an' tuck up de bank an' inter de bresh 'fo' Efalent miss him outen de year. He curl his trunk roun' an' feel fer him, but he kain't fin' him. Den he think mebbe he done fergot w'ich year he putt him in, an' so he feel in tu'rr one. Naw, suh, he wan't in tu'rr one, neener. 'Well, ef dis don' beat my time!' sezee; 'I sut'n'y putt dat owdacious creetur in my year. 'Tain' poss'bl' dat I cu'd go an' dream sech ez dat,' an' he stan' dar jes' a-huffin' an' a-puffin'.

"Hyar' he bin watchin' him an' he tuck de 'casion ter stick his haid outer de bresh an' say: 'To be sho' you wan't dreamin', Mistah Efalent; I wuz in yo' year, sho' 'nuff, an' a mighty bad time I have uv hit in dar. You axed me, suh. w'at wuz de marter, an' now I gwine tell you, dough I'se saw'y ef I seem onmannerly atter 300 


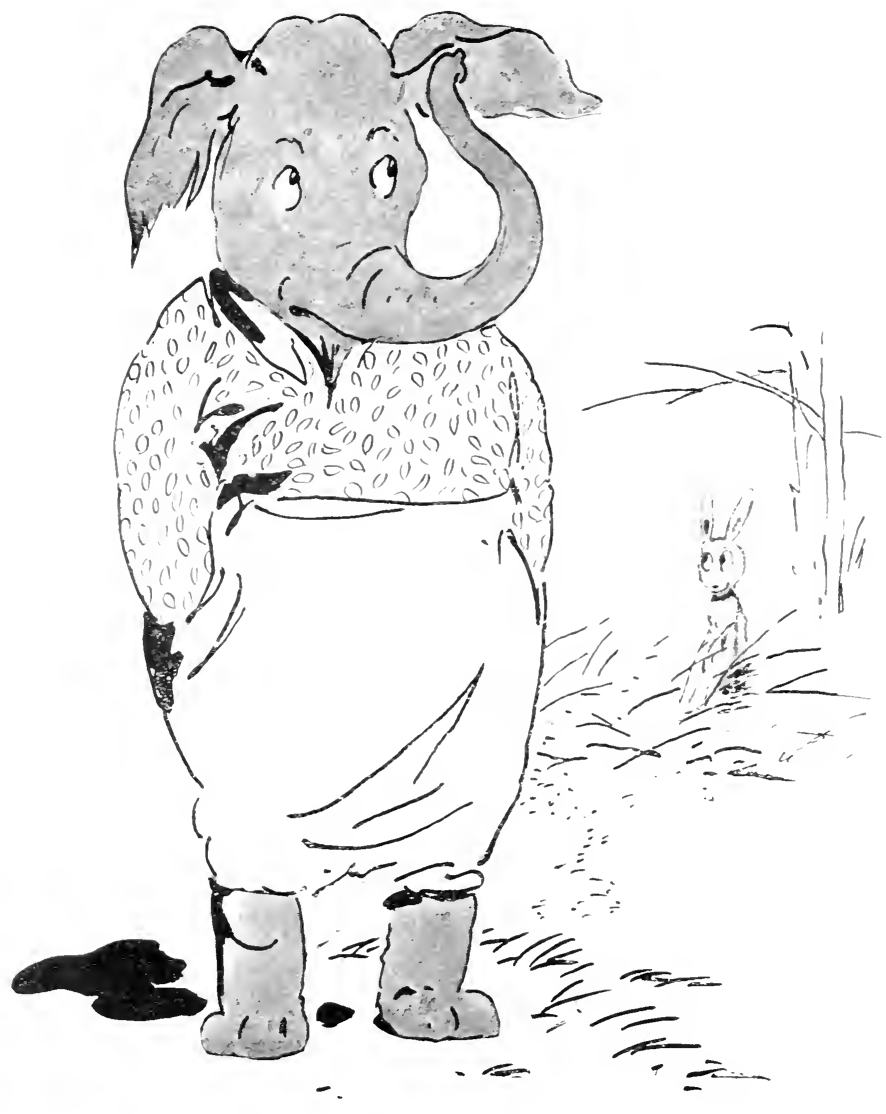

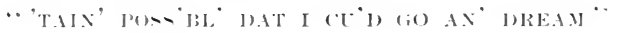





\section{MR. HARE AND IIR. ELEPHANT}

you teckin' me 'cross: but I'se 'bleeged ter say, suh. dat you done fergot ter wash yo' years dis mawnin', dat you has, an' I 'clar' ter goodness hit mek me feel plumb faintyfied in dar w'en you shet dat flap down on me; 'deed hit did. Nex' time you teck anyb'dy in yo' year, better see dat hit's clean. So-long, suh: so-long.' An' wid dat he shuk one'r his behime footses at de Efalent f'um roun' de cornder ur a tree an' wuz off in a jiff, leavin' dat gre't big creetur stannin' dar jes' a-trim 'lin' an' a-stompin' an' a-foamin' an' a-snortin' an' tearin' up trees by de roots, but 'tain' do him no good, fer Hyar' wuz outer sight by dat time, fur on his way ter de meetin'.

"An' now," finished Aunt Nancy. as she gave a last twist to one of Kit's long, yellow curls, "dat shows y'all w'at happen ter folks whar ain' tickler 'bout keepin' der years clean. Dey think no pusson gwine notuss, but someb'dy sho' ter fin' hit out an' mek fun ur 'em an' hol' 'em unp fer a disgrace ter der fambly an' de qual"ty in gin'l."

Here she carefully threw the combings that had accumulated in the making of the little boy"s toilet into the fire. explaining that if they were thrown away and not burned, the birds might find them and use them in nest-building, in which case the person from whose head the hair came would be sure to go crazy; nothing could avert the fate. 


\section{THE TOAD AND THE TERRAPIN}

Then Aunt Nancy had finished curling the small boy's hair, he insisted that she owed him another story, because she had not only hurt his ears, but had given his hair an unlucky pull. She objected at first, saying that she had other fish to fry and must go along about her work. At last she relented so far as to say that she would tell one if she could only think of something new. "I done tol' you so many tales," she said, "dat I done used all de creeturs up, runned 'em plumb inter de groun'."

"I know one you haven't told us about for a long time," said Janey, "and that's the Toad-frog; you told us a story about him a long time ago and I liked it. It was all about the Hopper-grass and the Chickenrooster."

Aunt Nancy felt flattered that her story should have made such a lasting impression, and it seemed to have a happy effect upon her memory, for she said at once that she believed she did recollect another story in which the Toad figured, along with the Terrapin.

"Hit wuz 'long in strawba' $y$-time," she commenced, "an' Toad-frog he say ter hisse'f dat he jes' natchelly 'bleeged ter have him a mess er ba'ies, 'kase he ain' had none dat 'ear, an' he wuz 'tickler fond ur 'em."

Here Ned interrupted to say, "Ho! Aunt Nancy, who ever heard of a toad eating strawberries?" 


\section{TIHE TOAD AND THE TERRAPIN}

"Well, dey does, den," said the story teller, indignantly; "I done seed "em at hit wid my own eyes; an" snakes does, too: dey is bofe mighty fond ur 'em. You neenter think folks is de on ies ones whar knows a good thing w'en dey sees hit. Does you s'pose de Lawd mek de good things jes' fer people an' don' want de creeturs ter git der sheer? Naw, suh. He want em ter go sheers; but folks done got so mean dat dey calls hit stealin' w'en de bu'ds an' de bees an' de wast-es comes atter de grapes an' de churries an' de ba ies an' sech ez dat.

"Well, I done tol" you strawba ies wuz ripe an' Toadfrog wuz jes" a-honin" fer some. He wuz gwine hoppin' down de road, singin" dis ol" song ter hisse"f:

'Dar u'uz a mouse live' in a house,

Wid a rinktum boddy middy kimo,

Dar un a frog live in a well,

Wid a rinktum boddy middy kimo, An' ef he ain' gone he live dar still,

Wid a rinktum boddy middy kimo.

Kimo-narrom, detto-sharrou,

Rinkitum boddy middy kimo,

String-strong pommy-doodle ally-mody dingdong,

Rinkitum boddy middy kimo.'

"Jes' ez he git dat fur along wid de song who shu'd he met up wid but ol' Mis' Tarry-long Tarr"pin. Now she wuz mighty fond er strawba ies husse'f, an' she knowed whar dar wuz a baid er nice, big ripe ones, an' she wuz right on her way to 'em den. Mos' in gin'l 303 


\section{AT THE BIG IIOUSE}

she wuz a turr"bl' slow trabeler, but dish yer mawnin' she wuz gwine 'long right peartly. W'en Toad-frog seed dat, he 'spicioned sump 'n wuz up, an' he say ter hisse'f, he say: 'Humph! ol' Mis' 'Tarr'pin sho' has got a move on husse 'f dis mawnin'. Now, wat is de meanin' er dat? Lemme see, she got a mighty good likin' fer strawba'ies, an' I boun' you she's right on her way to 'em dis minnit, ef de trufe wuz knowed. De ol' lady got lots er gumption, an' I reckon she know jes' whar dey're a-growin'. I gwine tackle her an' see ef I kain't git ter go 'long.'

"Wid dat he hop up 'long side er Mis' Tarr'pin an' he say, sezee: 'Heyo! Mis' Tarry-long, I ain' need ter ax you, how is you? you gwine 'long yer so gaily I sca'cely knowed you. I wanter ax you, ma'am, kin you tell me whar dey is any strawba'ies? I ain' had nair' one dis 'ear, an' my appentite gittin' so deliken dat I needs sump'n lak dat ter putt me in de notion er eatin' ag'in. You sech a smart lady dat I 'lowed you'd know whar dey wuz some, ef anyb'dy did.'

"Mis' Tarr'pin she wan't gwine gin her sekert away, so she let on she dunno nuttin' 'bout 'em an' ain' kyarin' fer 'em, anyhows. 'Sho! man, g'way f'um yer,' she say; 'w'at you reckon I know 'bout strawba'ies? I nuver eats 'em; I ain' got no mouf fer 'em.'

"Toad-frog seed he wan't gwine git nuttin' outen her dat-a-way, so he jes' mek up his min' ter co'te her a li'l an' see ef dat oon wu'k. 'A li'l co'tin' sometimes go a long ways wid a gal,' sezee ter hisse'f ; 'jes' a few li'l honey-wu'ds an' dar dey is.'

"He 'mence bein' mighty flattersome to her an' talkin' all sorts er sweet talk, lak men does w'en dey's 


\section{THE TOAD AND THE TERRAPIX}

co 'tin', dough dey mos' alluz 'ny hit, out an' out, atterwu'ds. Las' he up an' ax her ain" she wanter git ma "ied.

"She "low, 'Naw, suh, dat I don'; not ter no sech li'l jumpin' thing ez w'at you is.'

"Toad-frog langh fit ter kill hisse'f, an' he say, sezee: 'G'long wid you, gal, I ain' ax' you ter have me: I axed you does you wanter git ma“ied, jes' in fun, ter see w'at you gwine say. I ain' axin' none er de gals dese days: 'scuse me, of you please. Dis de time w'en you done hollered befo' you wuz hurted.'

"Dat mek Mis' Tarr"pin mad, an' she say: 'Hysh. man! g"long "way f"um yer; I done had nuff er yo" imp'ence. W'at's mo', I gwine tell er'y pusson I knows dat you done ax me ter ma'y you an' I oon have you. You spose any er de gals gwine look at you w'en I tell 'em dat? Naw, suh. W'en I p'int you out an' snicker an' snort an' say: "Look yonner, gals! Yonner goes my leavin's.",

"'Tas'm, dat dey is,' sezee; 'men-folks ain' so plenty dat gals kin give dersefs too many extry airs. Sidesen dat, I kin tell my side ur hit: how you done mistooken a joke fer a sho'-'nuff axin'; an' den whar'll you be, Mis' Tarry-long?'

"At dat she turn her back on him an' walk off. Toadfrog he ain' say nuttin' mo', 'scusin' ter tell 'er 'so-long.' Den he mek out lak he gwine hop off tu'rr way, but, bless yo' soul, no sooner wuz her back good an' turnt dan yer come Toad-frog, w'ich his tu'rr name wuz Jimmy Jump-er-long, an' gin a li'l jump an' dar he wuz, settin' up on her back, ridin' 'long jes' ez cool ez a cowcumber, an' ol Mis' Tarr'pin nurer even 'spicion he wuz dar, 'kase her back so hard she kain't feel him. 


\section{AT THE BIG HOUSE}

"Dey went "long dat-a-way, Toad-frog perched up dar cuttin' all sorts er shines, doin' lak he wuz clickin' his mouf at a hoss, widout mekin' no soun', an' stannin' on one foot, wid tu'rr laig stuck up behime in de air, fer all de worl' lak dem bar'back riders at de succus.

'Las' Mis' Tarr'pin she stop, an' de place whar she done turn inter wuz nuttin' mo' ner less dan a strawba'y-patch. Toad-frog hopped down, an' sezee, 'Kreech! kreech! kreech! I'se ma'ied a'raidy, so how cu'd I ax you ter ma'y me? Tell me dat, ma'am.'

“Mis' Tarr'pin sut'n'y wuz s'prise' w'en she see dat creetur hop down f'um her back. She say: 'Name er common sense, how you git on my back, you owdacious vilyun? Ma ied a'raidy, is you? Well, I sut'n'y is saw' $y$ fer de ooman. Who is she, anyways? I ain' nurer year tell uv her.'

“' 'Kreech! kreech! kreech!' sez de Toad-frog, sezee. 'Tain' no ooman 't all. I'se wedded ter dese yer ba'ies; dey is my fus' an' my las' an' my on 'ies love,' an' wid dat he fall ter eatin " hard $\mathrm{z}$ he kin swoller.

"Mis' Tarry-long Tarrpin she say ter herse'f, she say: 'I'm er min' ter gin “im one good bite dat he kain't fergit in a hurry, but I 'clar' ter gracious, I'se dat disguss'id dat I ain' g'wine sile my mouf wid him. Nemmine, Mistah Jimmy Jump-er-long, you stuffin' yo'se'f full er my ba ies an' you feel mighty smart orer hit, but some er dese days I gwine mek you smart on tu'rr side yo' mouf.' Wid dat she walk off an' leave him eatin', an' 'twuz good thing fer him she did, "kase, I tell you, she wuz a nipper; an' ef she d a-bit him he oon bin able ter fergit hit in a mont" er Sundays." 


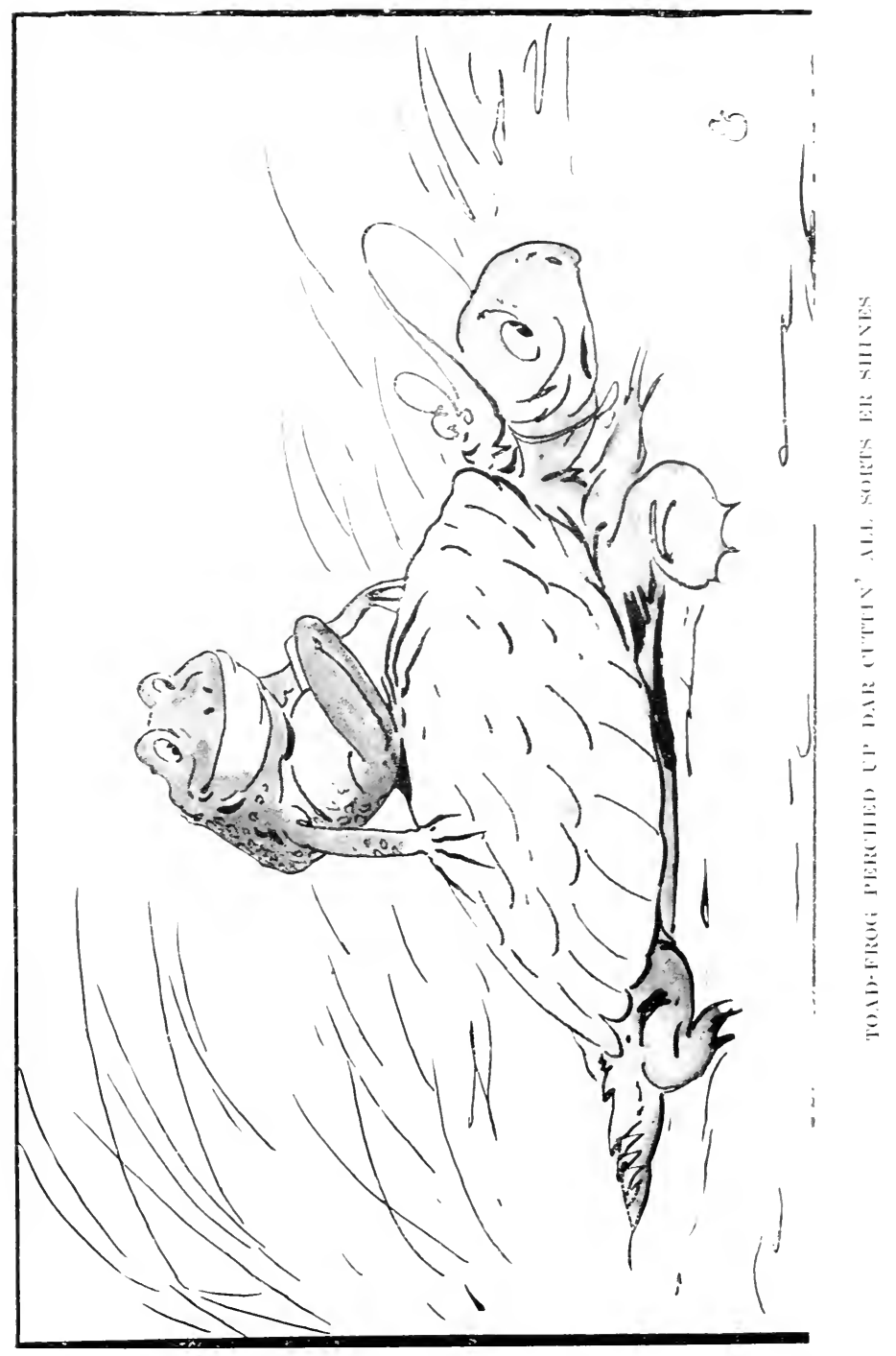





\section{THE TOAD AND THE TERRAPIX}

"Aunt Nancy," said Janey, "what did the toad mean when he said "kreech! kreech! kreech"?"

"Oh, dat 'uz jes' his way er talkin'," said the old woman.

"I never knew toads made any sound at all," said the little girl.

"Oh, rals, dey does; hit's jes" lak I tells you: 'Kreech! kreech! kreech!" sump'n "twix' de chu"ppin' ur a bu'd an" de skreekin ur a mouse. Many's he time I bin down cellar an think I year a mouse skreekin' an' git all skeert an' flutterated, an' yer all time 'twuz nuttin' but a li'l toad-frog whar wuz tryin' ter git outen my way." 


\section{THE IIOCKING-BIRD AND THE DRY-FLY}

One crisp day in late October Aunt Nancy elected to "go to mill." To this end she borrowed the plantation cart and the plantation mule, old Dick, known to Aunt Nancy as "Dick-mule." The children were invited to go along and joyfully accepted the invitation, for cart riding was an entirely novel experience to them. Ned sat on the seat with Aunt Nancy and was allowed the privilege of handling the whip; no easy task indeed, for Dick was a slow traveler and needed much urging, seeming to lose himself entirely, every now and then, in little naps by the way, from which he awoke with a start as Ned applied the whip. In fact, the little boy's arms ached before long and he gave relief to his exasperation by breaking into doggerel to this effect:

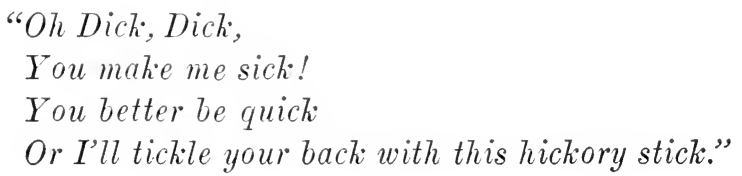

But eren this effusion, punctuated by blows from the whip, had no effect on Dick; he was too old a stager for that, and he calmly went on his way, giving no sign that he was aware anything else was expected of him. Dick was a well-known plantation character, a privi308 


\section{THE IOCKING-BIRD AND THE DRY-FLY}

leged one at that, whose ways were familiar to Aunt Nancy; so she finally said: 'Mars' Ned, you mought ez well putt down dat hick'ry; tain' no use. Dick-mule gwine jes' de way he wanster; you kain't hurry him; you jes' was'e yo' bref an' yo' strenk. He nurer 'spec's ter do w'at folks want him to: dat ain" mule manners. But mebbe you'll see him humpin' hissef on de way home, ef he gits strucken wid dat notion an' thinks you done gin up de hope er mekin him go." So Ted ceased to ply the hickory and presently gave up his high perch by Aunt Nancy to sit with the other children on the straw at the bottom of the cart, whose motions, though jerky in the extreme. had for them the unfailing charm of novelty. The road wound for miles beneath the tall pines, down, down into the depths of the woods, where it brought up by a deep, dark, still pond, whose existence was unguessed until you came full upon it. Here was the mill to which all the corn for miles around was brought for grinding, a rery primitive affair indeed, whose counterpart is yet to be found in some of the rural districts of the South. The water that furnished the motive power was conducted from a higher level, for at least a hundred yards, in a narrow wooden trough in which were several rude combs to catch obstructions, to a tall flume down which it fell on the wheel. The machinery was of the rudest, in keeping with all the rest. The miller was given no fee in money, but receired, instead, a certain portion or "toll" of the grain ground. This he measured ont in a wooden measure which was known as the "toll-dish." Rude and simple as the machinery is, the corn-meal turner out from these primitive mills produces bread of a sweetness and 309 


\section{AT THE BIG HOLSE}

freshness of flavor unknown elsewhere. one secret of which is, no doubt, that families prefer having the grinding done in small quantities so that the meal may always be fresh.

After the children had examined the mill and made the miller's acruaintance and eaten the luncheon bronght with them, they settled down to wait for the grinding of Iunt Nancy's meal, for several people had arrived before her and she was obliged to await her turn. Meanwhile she offered to beguile the time by telling the little folks a story.

"Hit's 'bout de Mockin'-bu'd an' de Dry-fly," she began. "One summer Mistah Mockin-bu'd jes' mo`n bin havin' a good time. He set out in de woods an' sing an' holler an' whustle an' go lak de cat-bu'd an' de jay an' ev'y urr kind er bu'd, an' he fool 'em all; dey s'posen 'twuz der mates callin' 'em, an' yer dey come a-flyin'; an', bless goodness, 'twan't nuttin' but ol' Mockin'-bu'd jes' a-settin' up dar laughin' at 'em. Sometimes hed go lak a hull nes'ful er young bu'ds w'en sump'n done got atter 'em, an' den de ol bu'ils 'nd come a-twitterin' an' a-fussin', skeert outer der wits, an' lo, beholst you. "twan't nuttin" but him ag"in. Tu'rr bu'ds git clean wo" out wid him an' dey ses ter one nu'rr. 'Temmine, dat ol' bag-er-shucks gwine git his comeuppance yit, you see ef he don': ef 'tain' one way hit'll be anu'rr: you min' dat. now."

"Nex', he pick hisseff up an' fly off to'des de white folkses" house an" perch hisse' $\mathrm{f}$ up on a tree in de vard. Fus' he'd go lak a dog, an' de cat she'd hump her back an' climb up on de smoke-house ter git 'way f'um de dogs; an' den he'd go lak a li’l young kitten cryin', 


\section{THE IIOCKING-BIRD AND THE DRY-FLY}

an' de cat she il skite down offen de roof to her kitten. Den he d go lak a dog ag"in an she d mek fer de roof onct mo". He kep' dat up 'twel he got her fair' "stracted. but las" hit come inter her min' jes' ter teck de kitten in her mouf an climb up on de roof wid hit. Mockin'bu'd he see he kain 't pester her no mo'. so he "mence ter cry lak a li"l chickin wid a hawk atter hit, an' rer come Mis' Hen wid her fedders all rufled and her wings spread out. an yer come de white folks wid a gun ter shoot de hawk. an' "twan't no hawk ter be seen. He fool em all so often dat he got em disgus: id, same ez he done de buids. De white folks wuz gwine shoot him, but one ir de white gals whar wuz'harin' a beau co te her 'bout dat time. say dey 'bleeged ter spare him, 'kase he come an set on de chimbly 'mongs' de irry-rines an' sing so sweet in de moonlight.

"Well, de wedder git hotter an" hotter. an" las' "long come Mistah Dry-fly jes" a-hollerin' an' a kyaryin on, day in an' day out. no let up: soun' fer all de worl' lak a ol' rusty buzz-saw. Mockin'-bu'd no sooner rear him dan he say ter hiso'f. sezee: 'Hi ' yi! w'at kind er noise is dat? I mus twis' my mouf roun' dat soun' an' den do a lill projeckin wir hit 'mongs' de creeturs.'

"Wid dat he twis" his mouf up de way he think hit orter be, but lawsy! 'tain' soun' no mo' lak Dry-fly dan I does dis minnit. He set his haid on one side an' lissen ağin an den try onct mo* Huh-uh! wusser dan befo". He whustle an' he bubble an' he nigh mos' bus' his thoat, but "twan't no nse.

"By dat time tu'rr bu"ds fin" out w"at he'z up to, an" dey ga'r roun an' lisen at him. an' dey wuz dat tickelt at him dat some un em come mighty nigh tum- 
blin` outer de trees, dey laugh so hard. Dey poke one nu'rr in de side an' ses: 'Hysh! g'way f'um yer! ain' I tol you he gwine meet his match some day? Keep hit up, Mistah Dry-fly; don' you stop a minnit, fer ef you do, dat rambunkshus bu d'll fall to an' mummick we-all ag"in."

"Wid dat, Dry-fly he goo on jes' a-buzzir' an' a-buzzin', louder'n urer; but sho! he ain' need ter do dat, fer Mistah Mockin-bud wuz dat tuck down in his fedders dat he jes' went a-slinkin' off inter de woods an' stayed dar, an' he sca'cely opened his mouf all endurin' de summer, 'kase he wuz so 'shame' he done met up at las' wid a pusson whar he kain't mummick. Tu'rr bu'ds dey flew'd roun' an' 'jyed derse'fs might'ly, mo'n dẹ done fer a long time, singin' an' chu'ppin', 'kase dey knowed no pusson wuz gwineter mek a mock ur 'em.

"Las' de fros' come, an' Dry-fly he tucken his buzz an' went som'ers wid hit whar 'twuz wo'mmer, er else de col' done kill him off. Anyways he stop buzzin' wid de fus' fros', an' den yer come ol Mockin 'bu'd, mockin' an' trickin' same ez urer an' singin' 'way thu de lightnights fer de gal an' her beau 'twel dey mos' thought summer done come ag'in. 'Laws-a-musey!' sez de gal, sez she, 'ef yer ain' dat sweet li'l bu'd singin' ag'in. I lak ter know huccome he stop so long. Ain' he got jes" de nices' li'l roice in de worl"?"

" 'Naw, dat he ain', sez de man, sezee: 'I heap ru'rr lissen at you talkin', Den de gal she giggle, an' I dunno w'at else dey ses, mebbe not much, but de bu'ds wuz jes' a-gabblin' 'bout ol' Mnckin'-bu'd.

" 'Uh-huh!' dey ses, 'yer he is hack ag'in. big ez life an' twict ez natchel. He'z got de imp'ence er de Ol' 312 


\section{THE MOCKING-BHRD AND THE DRY-FLY}

Scratch hissef. But tlat de way wid dese yer Mistah Brags: w'en dey meets up wid der match dey shets der moufs mighty tight, but w'en der betters go 'long an' leave em ter deyee'fs ag"in, sho! dey open der moufs so wide an' hollers so loud dat decent folks kain't stay whar dey is.'

"An' wid dat dey all went twitterin' off an' lef' him ter hisse'f. Trer sence den, 'long w'en de wedder git right wo'm an' de dry-flies 'mence der buzzin'. ef you'll pay 'tention you'll notuss dat de mockin'-bu'ds lays kind er low an' lets on lak dey think de wedder too wo'm fer singin'." 


\section{THE S.LCY YOLNG FROG}

"Tell us anuzzer "tory, please?" begged little Kit, as they still sat waiting for the miller to finish grinding Aunt Tancy"s corn.

"W"at about?" she asked.

"Oh, some animal you haven't erer told us about before," said Janey.

Aunt Nancy pondered a while. "I dumno w"at dat "nd be," she said. "Pears ter me I done gin all de creeturs a whu'l." After a little more thought, she said: "Dat pond putts me in min" ur a tale bout a frog whar live in jes' sech a place ez dat. I ain' nuver tol' you bout no frogs."

"You told us about toad-frogs," said Ned.

"I knows dat," she said, "but dem ain" frogs. Frogs live in de water an' toad-frogs live on de lan'. Well, onct dar wuz a ol' frog whar live in a pond all by hisse'f. 'Twuz way off, down in de woods, jes' lak dis. an' dark an' still, jes' lak dis. 'Twuz full er fish, an' de ol' frog he wuz a gre't fisherman an' ketched so many dat he got big an' fat an' his roice got loud an' deep, so't you cu'd year him mo'n a mile off. All thu de summer erenin's he kep' up his talkin', so loud you cu'dden year nuttin' else, an' hit mek folks feel right lonesome w'en dey wuz settin' by derse'fs in de dark. Dey cu'd year him at hit long ez dey kep' awake, 'jug-jug-er-oom, 


\section{THE SALCT YOLAG FROG}

jug-jug-er-oom, jug-er-oom, jug-ep̣-oom, jug-er-oom!" Ery now an den der'd rear a big splash. Dat "uz w'en he il go up on de bank an teek a han"-spring down in de water. fer he wru a gret han fer divin'. Hed come down wid a gret her-chug: an' den you culd year him jes" a boomin' an a-bellerin' umner de water.

"In de dartime he d come out on de bank an" sun hisse'f an' set up dar winkin' an' blinkin' 'twel he fell fas' asleep. One day wile he wuz teckin' a nap on de bank, yer come long a sassy roung frog whar bin nuttin' but a li'l tadpole dat re ' spring. He wuz new ter bein' a frog. an' he putt on lots ur airs dat wuz boun' ter be tuck outen him sooner ar later. He wuz mighty proud ur his new green suit wid de white res', an' he wanter g'long som ers an' show hisse f off. so he say ter his mammy: 'Мaw, I'se mighty tired er starin' in dish yer li'l ol' pond. I wanter go som ers an' do sump’n. You kain't 'spec' me ter grow up nuttin' but a numbskull in dis ol mud-lole. I houn' you. dar's heap nicer ponds dan dis somers, an I jes gwineter hop right 'long an fin "em. So many lil ol talpoles in dish yer pond dat I 'dar' hit goes "gins' de grain fer me ter stay yer an" sociate wirl 'em.'

"'Humph!' his mammy say, 'seem ter me rou gittin' too big fer yo britches. You wuz bawned an' bringed up wid de res' er my fambly in dis pond, an hit's plumb good 'nuff fer you. Iou gittin' mighty uppish, turnin' up ro' nose at de tadpoles, w'en you bin one yo se’f a li'l wile back.'

"Yas, dat I wuz, mammy.' sezee, 'but you see I ain' one now, an' dat mek all de diffe'ns in de worl".

"Mebbe you gwine fin' out dat dar's wuss things in 315 


\section{AT THE BIG HOUSE}

ponds dan tadpoles,' sez she; 'mebbe you mought be right glad ter git back safe an' soun' ter yo' fambly.'

'.'Ill teck de resk er dat,' sezee, an' wid dat he clim' up de bank an' went hoppin' off ter sce w'at he cu'd see.

"Well, I done tol' you he got ter de pond whar de big frog live an' seed him settin' dar in de sun, fas' asleep. He cas' his eye down inter de pond an' see 'twuz full er fish an' he say: 'Oh me! oh my! wish 't I'd a-brung daddy an' mammy an' de chillen. Now ain' dis jes' gran'! I gwine ketch me a good mess er fish an' tote some un 'em home an' show mammy I kin do sump'n, ef 'tain' bin long sence I wuz a tadpole. I don' reckon de ol' gemman snoogin' yer on de bank gwine kyare ef I ketch some, he got so many in dar. Kain't he'p hit ef he do kyare.'

"Wid dat he lit inter fishin' an' ketched a right good mess. Jes' 'bout den de ol' frog woked up an' seed w'at de young un wuz doin'. He cu'dden sca'cely b'lieve his eyes, an' he rub an' rub 'em ter mek sho' dar ain' no misteck.

"Young-frog he wuz talkin' 'way ter hisse'f, sezee: 'Dar, now, jes' one mo' fish an' I'll be thu an' teck myse'f off befo' ol' man Bull-frog wake. Mammy she'll be right proud ur me, dat she will.'

'Jes' den ol' Bull-frog riz up an' tuck him by de shoulder, an" sezee, in a turr'ble roice, an' swellin' up de big noise-bag beneaf his th'oat: 'Ol' man Bull-frog, hey? Dat w'at you call me! Well, you mus' be mighty young an' green not ter know better dan ter come yer an' teck fish f'um my pond. I don' 'low no one ter teck fish f'um my pond. I don' 'low no pusson ter fish yer but 


\section{THE S.ALCT YOLNG FROG}

myse'f. Now wat 'scuse kin rou mek fer yo'se'f, comin' ver stealin' fish w'en you see me fas' asleep?'

"Young-frog he gin a gre"t jump w'en Bull-frog clapped his han' on him, an' his eyes got mo' popped dan dey wuz befo". He let a fish drap outen his han', an' he gin de bigges' grin he cu'd wid lat wide mouf er his'n, an' he say, sezee: 'Shucks! is dis ro' pond? You sut'n 'y mus 'scuse me. Dis is de re $y$ fus' trip I urer tucken f"um home, an' I spose "twuz the same yer ez 'tis in ow' pond; er vbidy fish dar dat wanter.'

“ 'Naw, siree!" sez Bull-frog, "not by a long shot, suh. Dish yer's my pond, dese rer my fish, an' wen you fish outen dar hit's plumb stealin': dat's w'at 'tis.'

"You don' seson!"sez Young-frog, sezee, jes' ez sassy ez you please. "Well, ef dat's de case, suh, I kin jes' drap de fish right back in de pond an' g*long. I s'pose dat'll mek hit all right.'

" 'Naw, dat hit won't!" sez de ol' feller. 'S'pose I let you go, how I gwine know you won't come back yer ag'in an' bring all yo' fambly wid you an' all git ter thievin' in my pond? Taw. suh. wen I gits my han' on a thief I mos' in gin'l keeps hit dar. Yas, suh, I gotter putt you ter def. Now de queschin is, how I bes' do dat?

"Young-frog he say ter hisse"f: "'M! I b"lieve mammy bout right. I wish 't I wuz safe at home dis re'y minnit, stidder in de han's er dish yer pop-eyed, big-moufed ol bladderskite. No use ter baig him, I see he ain' gwine let me off. I ain' gwine let on I'se 'feard, neener, 'kise dat 'ud gin him too much sassifaction. I mus' jes' putt on de bes' front I kin an' mebbe I'll git 'way f'um him rit.' 


\section{AT TIIE BIG IIOTSE}

"Den he say out loud: "Well, suh, I 'spec' you'se right 'bout dis. You'se older"n me, an' I ain' gwine "spute wid yoll. Nammy done l'arn me I mus'n" argyfy wid follis older'n w'at I is. So you jes' teck me 'long an' do wat you think bes" wid me."

"All right," sez Bull-frog, sezee. 'Tin you gimme any notion er w" at be de mos" sho"es" way ter git shed ur you, "kase I wanter mek an" een" er dis; I ain' wanter do de job over ag in.'

"Toung-frog think he see a chanct right dar fer gittin' 'way, so he say: "Well, suh, you mought try one way an' den ag"in rou mought try nu'rr. Heaps er ways, but wat does you think bout thowin me in de pond? Dat seem ter me a right easy way, an" sho", too, 'kase I ain" lar'n teir swim yit."

"Te"ll see 'bout dat, sull, later on,' sez Bull-frog, sezee. 'Jes' putt on yo' thinkin' cap ag' in an' tell me some urr way dat I kin try, fer I ain' so sho" but w'at rou kin swim; you done bin a tadpole, an 'dey all kin swim.'

"Done fergot all I knowed w"en I wuz a tadpole.' sez Young-frog, sezee, 'an' I ain' ketched up wid de ways er frogs yit.'

"'Temmine,' sez Bull-frog, sezee, 'we'll see 'bout dat ag"in. T'ell me some urr way"

"Toung-frog he study an" study an" las" he b"lieve he see nu'rr way out. He say: "Well, ef you'll jes' go over dar an" git dat piece er rock an" smash hit down on me yer whar I set on dis rock, hit'll een me up in no time 't all. Blip hit down, an' hit"ll jes" smash me flat ez a pancake.'

"Bull-frog clap his han's an' say: 'Dat's de ticket! 318 


\section{THE SALCY YOLNG FROG}

You sut 'n'y got some breens in yo" haid, ef you is young an' green. Yer's whar I gwine do lak you ses. "Twon't teck me but a mimnit ter fetch dat rock, an' fo" you know hit you won't be nowhar's.'

"Bull-frog hop ofl atter de rock, an', bless goodness, his back wuz no sooner turnt dan Young-frog jes" gin one gre't spring an' lan'ed in de mitdle er de pond. He went swimmin' off ter tu'rr side an’ wuz up de bank by de time Bull-frog got back wid de rock.

"Bull-frog come "long back, talkin". He say" 'Juger-oom! Jug-er-oom! Jug-er-oom! Now git a good raidy, young feller, fer yo time done come - an" den he see de place wuz emptr. "Whar on yearf is dat pesterin' li'l thief er de worl' gone ter?" sezee, "jug-er-oom! juger-oom! jug-er-oom!'

"'Gone inter de water, suh,' sings out Young-frog f'um tu'rr bank. 'I wuz bawnded an bringed up in de water, an' my daddy an' my mammy befo" me. Tre is all fus'class swimmers, an" you orter knowed better dan ter lef' me settin' dar 'long side er de water. I gemman ol' ez w'at you is orter be mo' up ter smuff dan a young frog whar wuz muttin' but a tadpole a li"l w"ile back. So-long, suh. I gwine home ter tell dardy an' mammy an' all de frogs in ow' pond jes' whar dey kin git 'em a good mess er fish. So-long, suh, we all be orer ter see you some er dese times w'en de walkin's goor an' de light nights come.'

"Y'all kin see f'um dat," added the story-teller, "w'at resks chillen run dat don' min' der mammies an" stay home an' be sassified, whar dey's tooken good kyare ur. An' now yer come de man wid my meal. an' yon's Dickmule jes' natchelly pawin' up de groun', so I reckon we 319 


\section{AT THE BIG HOUSE}

bes' pile ow'se'fs inter de kyart of we wanter git home 'fo' sundown, 'kase dar's no tellin' w'at Dick gwine think 'bout hurryin' hisse'f. Nuttin' mo' onsartin in dis worl' dan de doin's uv a mule. He dunno hisse'f, f'um one minnit ter de nex'." 


\section{THE CRANE AND THE HUMIIING-BIRD}

The long visit to Uncle Henry was nearly at an end. Next day but one the ehildren and their mother were to go North again. So it eame about that the little folks were once more permitted to spend the evening at the cook-house, where there was a goodly gathering, presided over by the little hunch-backed cook, Eliza, who, as she would have said, had sent out "noration" that there was to be a grand story-telling for the benefit of the little white children who were so soon to leave them. Sam, the driver, was there, beaming on his elderly enchantress with every white tooth in his head. Tim, the plow-boy, had brought his banjo and was happy in the presence of his eoy charmer, Cassy. Aunt 'Phrony's grandson, "Wi'yum," was also present, while Coonie, the house-boy, constant in his devotion to everything eatable, was seated on the hearth engaged in roasting potatoes in the ashes.

Of course the two story-tellers, Aunt Nancy and Aunt 'Phrony, were present, for the oceasion would have been as nothing without them. These last were rather shy of beginning, saying that they "wan't feelin" quite up ter hit yit," so, by way of raising their spirits to the desired pitch, Tim picked his banjo a while, Wiyum did the famous "back-step" and Connie executed a curious dance, the performance of which he called "knockin' 


\section{AT TIIE BIG MOTSE}

de "rang-a-tang," Juring which he thumped his feet on the floor in such an emphatic manner that it seemed as if something must give way-either feet or floor. But neither did, and the dance reached a safe conclusion amidst a great deal of applause and laughter.

At last the turn of the story-tellers came, and Aunt 'Phrony allowed herself to be persuaded to begin. She said she would tell the story of the Humming-bird and the Crane, as it contained some good adrice to young men who were courting, and here she rolled her eyes in a meaning way at Tim and Cassy.

"I reckon," she began, "dat mens bin co'tin' gals urer sence de beginnin' er time, an' I een-about reckon dey gwine keep hit up ontwel de een'. W'at's mo', de gals alluz bin rumnin' on wid 'em, laughin' at 'em one time, cryin' 'bout 'em anu'rr, turnin' der backs on 'em one minnit, rumnin' atter 'em de nex'; an' I s'pose dey gwine keep up dat foolishness long ez de worl' wag. Back in de ol' days, dar wuz a gal whar live way out yonner som'ers; a mighty harnsome, likely sort er gal, an' all de creeturs wuz plumb crazy over her, an' 'spute an' kyar' on 'bout who gwine git her, an' sometimes dey had reg'ler fist-an'-skull fights 'bout hit. De gal she wuz mighty nice to 'em all, rumin' on wid 'em an' sof'sawderin' each one w'en she git him off by hisse'f, 'twel he think he'z de one she gwine choose, fer sho'. Dat-away she keep 'em all projeckin' roun' her day in an' day out, 'kase she thought she cu'd git ma'ied any time she git raidy ter quit her foolishness, jes' de way dese yer good-facetet gals is mighty ap' ter think dey kin' do, dough sometimes dey keeps hit up jes' a li'l too long an' fines derse'fs high an' dry on de she'f. Las' her folks 


\section{THE CRANE AND THE HLMIINAG-BIRD}

git 'feard dat mebbe dat de way she gwine do, an' dey tell her she mus" mek her ch"ice an git ma"ier.

"De bu ds dey wuz kind er lis'nin" rom", a " der got de news er dis firs', an' dey ses ter one nu': dat dey gwine 'cide de marter wid a race, startin" "wa off f"um de gal's house an "w'ichurer got dar fus' wo: ter have her.

"Gal she ain' say nuttin", jes" grin at "em all lak she alluz do, an" doy g"long off ter de startin" plare jes" mo n "sputin' an argyfyin' wid one nu'rr. Las" dey got so rantankerous dat mos" un em wuz plumb "eard ter go inter de race fer fear dey mought be tore limis fum limb ef dey did git de gal. Las" dar wan't but thee lef" dat wuz willin ter mek de trial, an dem wuz de Tukkey an' de Crane an de Hummin'-bu'd. Tukker he start off mighty biggitty an flewed a li'l ways at a bic bus' er speed, but he wuz too hairy tor keep dat up long, so he lit an' tries ter run 'long de groun', but sho! he dore fergot de time de cunjerers putt a lot er li"l bones in his laigs so"st he cuidden run fas". He seed tu'rr bu'ds ruz gittin' so fur ahaid ur him dat 'twan't no use ter try. so he say ter hissef, sezee: 'Shucks! let me outen dis: I ain' want nuttin' ter do wid no sech a hurrah's-nes' ez dish yer is: dar ain no gals livin' dat's wuf hit. Lemme git back ter my home an stay dar in peace an' quiet.' So he went swingin' off home. an' dat lef' nob'dy in de race but de Crane an' de Hummin'-bu'd.

"De Crane mighty sho' he gwine beat. kase he have sech long laigs, an' Hummin'-bu'd sho' he cu'd beat. 'kase er de swif'ness ur his wings. Crane say ter hisse'f. sezee, 'Humph! I ain' got dese rer long laigs fer nuttin'; I gwine beat dat li'l no-krount HImmmin'-bu'd ter 


\section{AT THE BIG HOLSE}

flinders,' an' Hummin'-bu'd he say ter hisse'f, sezee, 'Shucks! how kin dat gre't, gawkin', long-legged creetur think he gwine keep up wid me? Hit's plumb rank foolishness fer me ter keep goin' night an' day, so I jes' gwine res' me off good at night, an' in de mawnin' I kin soon ketch up wid ol' Crane an' pass him on de road.'

"So Hummin'-bu'd he res' off all night, an' Crane he go on an' on, an' w'en mawnin' come an' he see dat Hummin'-bu'd ain' kotch up wid him, he say ter hisse'f, he say, 'Lazy li'l rascal. I boun' you he 'z sleepin' yit, an' I 'spec' de sun be good an' high beio' he crawls out, so I jes' gwine stop right yer at dis pond an' git me a snack er frogs, "kase dish yer night-trabel done wo" me to a frazzle an' lef' me empty ez a go'de.' Wid dat he stop at de aidge er de pond an' stan' dar on one laig, waitin' an' watchin' fer de frogs. He wuz a pow'ful good fisherman an' mighty fond er frogs. He cu'd spy 'ẹm an' run 'em thu wid his bill 'fo' dey knowed w'at had 'em. He stood dar stuffin' an' stuffin' hisse'f 'twel he clean fergot how de time wuz passin', an' fus' news he knew yer come de Hummin'-bu'd an' pass him lak a streak er lightnin'. He call out ez he pass, 'Heyo! Mistah Crane, teckin' hit easy, I see, suh. Dat's right; don' yo' hurry yo'se'f fer nuttin' ner nob'dy, let 'lone a gal. So-long, suh; hope you git dar in time fer de weddin'. Crane he hurry hisse'f might'ly atter dat, but he wuz stuff' so full dat trabelin' wuz hard wu'k, an' he ain' see Hummin'-bu'd ag'in dat day.

"Tell, dey kep' hit up dat-a-way, one stoppin' ter res' by night an' tu'rr stoppin' ter eat by day, 'twel de las' day done come. Crane he 'cide ter do widout a snack 


\section{THE CRANE AND THE HUMMING-BIRD}

dat day, an' he putt on a big bus' ur speed an' got ter de gal's house 'bout noon. Jes' a minnit atter, yer come

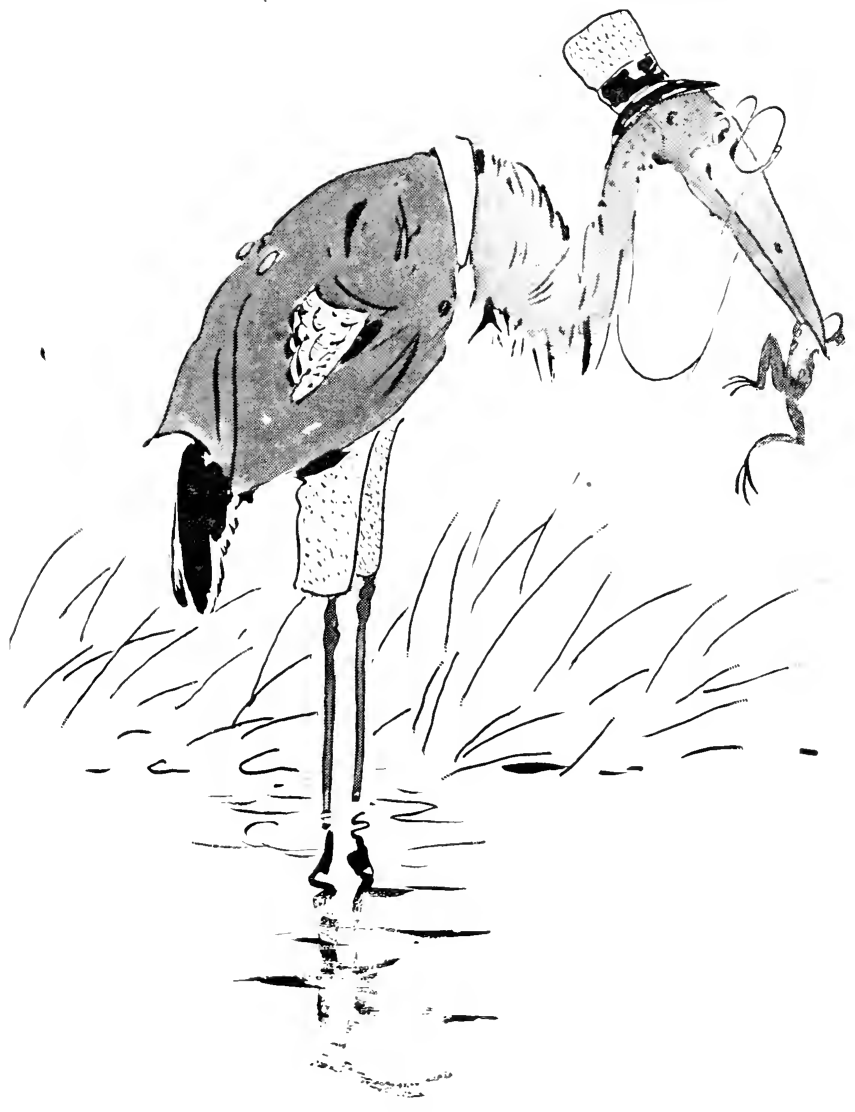

Hummin'-bu'd, all a-flutterin' an' a-flusterin', an' dat mad dat he ain' kin see straight, 'kase he los' de race 325 


\section{AT THE BIG IIOLSE}

jes" by dat one minnit. De trufe wuz he done orerslep' hisse"f er he moughter beat ol' C'rane widout half tryin'.

"Now de gal she wanted de Ifummin"-bucd ter beat, an" she wuz might "ly putt out w"en she see de Crane wuz de winner, so she mek up her min' dat she oon tek necner one ur 'em, an' w'en Crane step up ter claim her she say, 'Naw, suh, 'scuse me, suh. Nex' time you mek a bargum I rize you ter ax de cornsent er de lady. Yall tol me you gwine run a race, but is you urer year me say I gwine ma'y de winner? Naw, suh, I ain' say nuttin' 't all. JFistah Crane, yer, he done think heap mo ur his dimner dan he do er me, an' Mistah Hummin'bu'd think too much ur his natchel slumbers, an' you bofe done bin too sho' er gittin' me. You mus' nuver be sho" ur anything w"en you 'se co 'tin' a gal, leas" ur all. de gal husse'f. An' now I ain' gwine teck neener one'r you. I gwine teck dish yer King-fisher whar bin settin' yer jes" a-cotin' an' a-cotin' me w'ile y'all bin off on dat race.'

"So dat de way she tuck an' done, an" de Crane an' de Hummin-bud jes" hatter turn tails an go home, an' my daddy. w'en he tol' me de tale, say dat urer sence den. "w'en de medincin'-men cunjer ter fin' out who gwine may who, dey names de onlucky beads atter de C'rane an' de Hummin'-bu'd, an' de lucky bead dey call atter de King-fisher." 


\section{THE FOX AND TIE HOT POTATOES}

After the story of the Crane and the Humming-bird, Liza, plaring the rôle of impartial hostess, called on Aunt Nance to take part in entertaining the com. pany.

"Xaw, ma'um. Sis" 'Liza, please "scuse me dis erenin'," said she; "de mistry in my footes dat bad dey feels lak two plumb chunks er pain, an' my haid ache me so s't I kain't rear mrse'f think, let "lone study "bout sech ez dem ol" talcs. Tou please, ma'am, ter scuse me."

But the company fully understood that, like many an entertainer in more exalted circles of society, Aunt Sancy wanted coaxing, and between their compliments on the one hand and on the other sundry sniffs from Aunt 'Phrony, which seemed intended to cast discredit upon the old woman: miseries of head and feet, she was finally induced to relent.

She was about to begin, when she fell to langhing immoderately at Coonie. who had burned his fingers in pulling a hot potato from the ashes, and was sucking them rigorously with many grimaces and exclamations. Then she could speak for laughing, she explained that it had reminded her of one time when Mr. Fox pulled hot potatoes from the ashes for the benefit of Mis' Molly Hare and her family.

"'Twuz one winter night," she said, "one'r dem nights w'en ol' Jack Fros" jes' goes a-whoopin' an' a-hollerin' roun' de house, whustlin' thu de keyholes, an' rattlin' 


\section{AT TIIE BIG IIOT'SE}

de winders an 'retchin' up thu de cracks in de flo' ar' pinchin' folls by de toes. Ol' man Hyar an' Mis' Hyar' an' de chillen wuz all scrunched up by de fire tryin' ter keep wo'm, waitin' an' watchin' fer de 'taters whar wuz roas'in' in de ashes.

" 'Lawsy! lawsy!' sez de ol' man, stretchin' hisse'f an' knockin' de ashes onten his pipe, 'lawsy! lawsy! dar ain' nuttin' in dis livin' worl' gwine roust me outen de house dis night. I gwine stay right yer 'long wid y'all an' toas' my shins an' smoke my pipe. Hits col' 'nuff outside ter freeze de hawns offen a buff'ler. Ol' ooman. git out de cider-jug an' le's we have a li’l dram all roun' ter wo'm us up, an' by dat time de 'taters 'll be good an' done.' So Mis' Hyar' she drammed 'em all roun' wid cider, an' den she 'mence ter scratch out de 'taters, w'ile de chillen sot up on der ha'nches watchin' her wid der big, bright eyes an' wukkin' der noses an' smackin' der chops lak dey kain't sca'cely wait. Dat mus' 'a bin a sight, Mis' Molly scratchin' 'way in de ashes wid her li’l ol' white cotton tail turnt up in de air an' her behime footses jes' a-flyin'.

"W'ile she wuz wukkin', de chillen dey wuz grabbin' de 'taters, an' de ol' man he hatter do a li'l cuffin' an' scoldin'. He say, 'You Jumper an' Thumper! I want you ter stop snatchin' f'um Bumny an' Honey: dem's de babies, an' dey kain't look out fer derse'fs; you orter be 'shame' er yo'se'fs! Winker an' Blinker, you neenter think I dunno you'se snougin' 'taters an' hidin' 'em behime vo' backs. Putt 'em back an' go sheers all roun', er I come dar an' cuff you good, so he p me bob!'

"Mis" Hyar" she go on scratchin' an' she git mighty wo'm. Las' her footses 'mence ter smart, and she say, 
TIIE FOX AND THE IIOT POTATOES

'Lan' er de livin'! dish rer too hot for me ; lemme git out whar I kin cool my paws: nemmine of I gits de chilblains: mought 'z well freeze up ez burn up.'

"Wid dat she mok fer de do". an" w" "an der open hit, dar stood Mistah Fox, jes" a-shiverin' an" a-shakin' wid

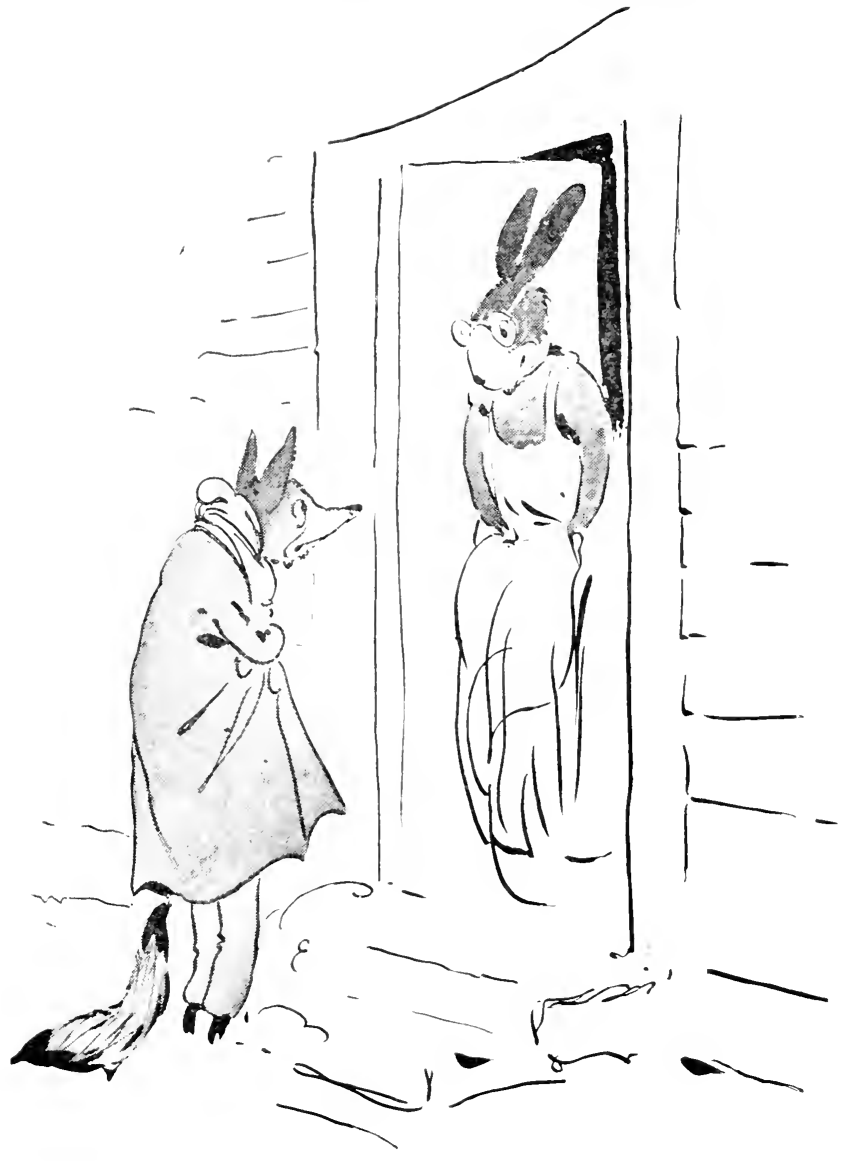




\section{AT THE BIG HOTSE}

de col'. Mis' Molly she say, 'ITeyo! Mistah Fox: dish ver"s a nice, wo m erenin you tone slected fer yo' visit. Will you have a cheer jes" ontride de do "?'

"Fox he say, wid his toofs jes" a-clatterin' an' a-chatterin', sezee, 'Please, ma'am. Mis' Molly Cotton-tail, ter lemme come in dis re y minnit an' don' stan' dar projeckin' wid me, 'kase I is friz ter de marrer an' I'se bleeged ter git thawed out er else drap right yer in $m y$ tracks.'

“ 'Tell,'sez Molly, sez she, 'I dumo 'bout lettin' you in; dish yer a mighty col night, an' we-all got jes' a hanful er fire an' a lot er paws ter be womed at hit, fer̈ you know I got some 'leren er mo' chillen in my fambly, but I reckon I mought mek out ter let you in ef rou'll unnerteck de job er diggin' a lot er 'taters outen de ashes.'

“'Lemme git in,'sez de Fox, sezee. 'I'se good fer all night at dat job; jes' lemme git my footses in dem ashes onct an' I ain' gwine teck 'em out in a hurry.'

"Hyar" she say, "Nalk in an' mek yo'se'f at home: we gwine gin you a mighty wo'm welcome,' an' right dar she grin behime Fox's back an 'wunk at ol' man Hyar'. 'Git yo' moufs raidy, now. chillen!' she sing out ter de young uns, an' ol' Fox jes' lit inter scratchin', lak all possessed. an' de chillen sot dar an' gobbled 'taters faster'n he cu'd dig 'em out. Las' he 'gin ter git kind er wo 'm. an' he say, 'Ouch! ouch!! ouch!!!' Mis' Hyar' say. 'M'at de marter, Mistah Fox, ain' yo' feet wo'm vit?'

"Fox he ain' say nuttin', jes' go on a-scratchin'. but pres'n'y his footses wuz dat scorched an' swinged dat he cuidren stan' hit, an' he say, 'Jimminy Crick! Mis' 


\section{TIIE FOX AND THE HOT POTATOES}

Hyar", you mus" let me ofl on dis job, you sho"ly mus". I reckon de chillen done got der fill by dis time, anyhow:

" 'Yaw, mammy, dat we ain'! mek him go on, dey sez. an "Mir" Itrar" she say, "You year dat now. I kain't let my chillen go hongry, so I bleeged ter ax you ter go on, er else me an my ol man gotter fling you out dar in de fros:"

"Fox he set an" study a minnit 'bout w'icht wuz de wuss. de fire er de fros", an' las" he mek up his min' ter try de fire a li"l longer. Sidesen dat he wuz in de hopes er gittin" a "tater fer his own sheer "fo" dey wuz all gone. So he go on wid de scratchin' fer a w'ile, an' las' he kain't stan' hit no longer an' he bus' out ter yellin', 'Ow' ow! ow! ouch! lemme outen dis! putt me in de fros' er any place whar I kin cool my footses! m-m-m-m-umph! my footses done bu'nt clean offen me!"

"Dat wuz de trufe, too. an" w"en Mis" Hyar" see Fox ain' got no footes ter scratch wid no mo", dey jes' tuck him an 'flung him out in de col' an' shet de do' on him. He went limpin long li'l wars. colder'n wer. an' jes" a-studyin' how he gwine git back by dat fire. Pres'n'y he met up wid nu'rr Fox, a li"l young feller. easy ter fool, an' he say ter him. sczee. 'Heyo, young man, w'at you doin' canterin' roun' dis col' night: does yo' mammy know yo'se out? You better come 'long gूo back wid me ter ol' man Hyar's. Dey got a rip-roarin' fire dar an' a lot er 'taters roas"in'. an' dey'll let you git wo'm an' gin you a 'tater, too. jes' fer pullin' 'em outen do ashes.'

"Mistah Youn - fox say. 'Tell. Mistah Slickry-sly, I ain' kyare ef I do.' So dey g'long back ter ol' Hyar's 
house an' knock, an' Slickry he 'splain dat he done brung someb dy ter pull taters onten de ashes, so Mis' Hyar' she 'vite 'em in an' set de young feller ter wu'k.

"IIe wuz mighty peart 'bout hit at fus', but I let you know 'twan't long 'fo' he slack up, an' pres'n'y he wuz grmutin' an' groanin' an' lickin' his pars. Ol' Fox he sot dar an' aigged him on an' et taters wid de fambly an' hollered an' laughed w'en las' Young-fox drap a hot tater an' bolt fer de do". 'Don' be in sech has'e,' he call out atter him; 'you gwine fin' mighty col' wedder outside.'

"Mis' Hyar' she putt on a sollum look an' she say, she do, 'Mistah Slickry Sly-fox, ain' you 'shame' ter mek fun er yo' feller creetur?'

"Fox he 'spon', 'Naw, dat I ain'. You done yearn befo' now dat mis'ry love comp'ny. You don' s'pose I wuz gwine be de on'ies" fox in dese diggin's whar go limpin' roun' wid stumps fer fontses? Naw, suh, not ef de co'te know hitse'f, an' hit think hit do.'

"Ol' man Hyar' let on lak he wuz clean outdone wid sech talk, an' he jes' tucken his foot 'way f'um Fox an' lan'ed him plumb outside de do', an' he say, sezee, 'Teck dat, now, an' g"long, an' don' you come yer no mo' talkin' no sech a way ez dat befo" my li'l chillen; I don' want 'em ter l'arn sech 'harishness ter der feller creeturs. You year me, suh! now mosey, befo' I teck my font 'way f'um you ag'in. I don' wanter hurt you, 'deed I don'.'

"Den he shet de do' an' come back, an' Mis' Hyar' she cuff de chillen all roun' fer scrabblin' in de ashes an' gittin' derse'fs dirty, an' den dey all went ter baid an' slep' jes' ez soun' ez ef dey ain' bu'nt de footses offen de foxes an' den turnt 'em a-lonse in de col'." 


\section{THE FCYERAL OF MR. DOG}

"Aunt Naner," aid Ned, after the story of the For and the hot potatoes, "do you remember telling us a story about Mr.. Dog and the way she chased old Mr. Fox? Do rou know any more stories about her?"

"Lemme see bout dat," she said. "Mebbe I does, but I kain't jes lay my finger "pun hit dis minnit. My backdars is gittin' way f'um me so fas' dat I hatter stop now an' den ter git a fraish holt on 'em, lessen I wants "em ter git clean out er sight."

She pondered a little while, with her head on one side, and then resumed: "Seem lak I kain't jes" 'zackly think ur anu'rr tale 'bout ol' Mris' Dog, but I does 'member one "bout de fun'l er Mristah Dog. One time noration wuz sont out thu all de kyountry dat Mistah Dog done daid an' IIistah 0 wl gwine preach de fun'l sarmint, an' er. y puson mus" come ter year hit. Some er de creeturs jes' jumped up an' down an' clapped der han's an' cut all sorts er shines, 'kise he lin worryin' an' pussecutin' an' chasin' 'em 'twel dey w'uz 'feard ter stick der haids outen der holes.

"S'so er'yb'dy putt on der bes' riggin" an' sot out fer de preachin'. Mistah Hyar' he g'long pas' Mistah Fox's an he sing out. 'Oh. Mistah Fox. is you yearn Mistah Dog done dair?' Fox he 'spon'. he in. 'Is Mistah Dog dain? I ain b'lieve dat; hits ton good ter be true. I know he gwine chase me many a time rit.' 


\section{AT TIIE BIG HOCSE}

"Yas," sez IIyar", sezee. 'hit's de gospil trufe. De fun'l gwine be preached dis day, an' you mus' come 'long er me.'

"So dey sot out fer de preachin', whar er'yb'dy wuz tryin' mighty hard to behave sollum. Owl he perched up on a branch uv a tree whar he cu'd look down on de folks, an' he putt on his specs an' cl'ared his th'oat an' coughed, an' den sezee, 'My 'specful yearers, we is all ga 'rrd toge 'rr dis day fer a sad an' mo'nful 'casion, an' yit not so mo'nful, neener, 'kase ef de trufe mus' be tol' - an' dat's w'at I'se yer fer, dear sinner fren's-I 'spec' mos' ur yon is rej'ycin' an' givin' thanks fer de takin' off er Mistah Dog, 'kase he wuz de foe ur peace an' quiet an" de natchel-bawn inimy ur er'y one'r you-all. Ez fer me, my min' done tore in two 'bout dis, fer I kain't rej'yce widout medjure; my inj'yment is chasten' by de fac' dat I has de hairy job er preachin' dish yer sarmint, an' yit I is sut'n'y glad he gone, fer he done done so much damagement an' kilt so many sheep an' hyar's.'

"Right dar ol' Hyar' he groan an' he say, sezee, 'Yas, Lawd!' an' de sheep he say, 'Trufe, too!' an' tu'rrs all jine in wid, 'Year dat wu'd, now, do!'

"Owl he go on: 'But li'l chillen, I gotter say unter you dat you has yo' lesson ter l'arn f'um de suddint teckin' off er Mistah Dog. How you know you is'n' gwine be tucken off, too, in de midse er yo' sins, in de same lak manner? Who kin tell dat to-morrer some er you won't be layin' whar Mistah Dog is, all strouded an' raidy fer de grave? I stan' yer lookin' each one'r you in de eye an' scannin' de continents er yo' face, an' I 


\section{THE FETERAL OF MR. DOG}

kin see that some er you is larin' right at de gates uv iniquity, kickin' yo' heels ter git in, an' I ses muter you, look atter de goin er yo" day's, right yer an' now. You gotter gin up all dese yer fine fancies or de worl, whar ain" wuff shucks, nohow, not eren w'en deys tricked out in pupple an' fine linen an' gilted all roun' wid gol'. Look on dis col' cawpe an' teck de warnin"' Tit I ain' grwine say he got no chanct. atter all. "kase he wuz a mighty gnod fren” ter Mistah Man, an" tuck good kyare er de honse by night an by day, an" dat sarrice gwine be 'membered. Yas. my fren's, I 'spec' Mistah Dog gwine sho"ly be sared atter all. An' now y'all kin sing sump'n an' den come up, one by one, decint an' in order, fer de las' viewin' er de 'awpse.'

"De creetur's go up an' look at him an' den come back an' whusper' "mong derse'fs. Wolf he say, sezee, "Tell, ef he is daid, I'se glad he daid, "kase he done chase me 'nuff, lawd knows! he orter been dair long 'go.' Tarr'pin he laugh, an' sezee, 'Mistah Owl done tol' us Mistah Dog sho'ly sared. Well, dat's mon Brer Wolf uver gwine be,' an' tu 'rrs all langh at de joke, an' all un 'em have sump'n ter say' 'bout Mistah Dog.

"Atter de fun't wuz preached dey wuz fixin' fer de burryin', an' Hyar' he say. sezee. 'Fren's. I he'p y'all wid pledjure an' be tickelt inter de bargum.'

"Wolf he say, he do. 'Dog-my-cats. ef I b"lieve he daid, atter all! I ain' gwine he p burry him.'

"Tarr"pin he say, 'I bin trabelin' sence befo' sun up: I'se too mawtul tired ter think 'bout han lin' dat kyarliss.'

"Mis' 'Possum she say, 'Well, you see I got all dese 


\section{AT THE BIG HOCSE}

chillen er mine hangin onter me by der tails. A ooman wid chillen settin' all over her back de way mine is kain t ve well he p wid de burryin:'

"Fox he say, "Y"all kin see fer yo"se"fs dat I got my footses mos" bu'nt offen me. A man all lame' up dis-away oon be much use ter you, dat's sut'n. Ef 'twan't fer dat I be glad ter he'p.'

'Dey all kep' on dat-a-way, mekin' 'scuses, 'twel las' Jar wan't none lef" but de Hyar" an' de Sheep, so dey s'lected dem out ter burry Mistah Dog, an' atter dey done digged de pit an' go ter putt him in. Hyar' he say, 'You go down fus', Brer sheep.' Sheep he say. 'Ba-a-a. I ain' blieve I kin, an 'wid dat Hyar" gin him a shove dat sont him down de hole, an' den he jumped in right on him, so's't de fall wuz mighty sof' an' easy fer him.

"All dis time Mistah Dog wuz layin' 'sentially still an' quiet, an' tu'r's picked him up an' passed him down de hole ter Mistah Hyar' an' Mistah Sheep, but Wolf he kep' a li'l ways off, 'kase he wan't so sho' Mistah Dog good an' daid.

"Dog he jes' bin a-lettin' on all dis time, an' w'en he wuz clean down de hole an' dey 'mence ter chunk dirt on him. he wake up mighty suddint. an' he say, sezee, 'Th-huh! gwine burry me, is y'all? Putt me 'way in de midse er my sins, is you? I let you know I ain' ez daid ez I look. Webhe some er you-all gwine have yo' fun'l sarmint preached befo' I needs one.'

"Wid dat he mek a grab at Mistah Hyar' an' Mistah Sheep, an' dat wuz de las' ur 'em bofe, fer dey cu'dden git outer de hole widout he'p, an' tu'rr creeturs jes' tucken ter der heels an' went a-scootin' widout waitin' ter see w'at happen, ol' Tolf at de haid er de gang, 336 


\section{THE FINERAL OF IIR. DOG}

'kase he bin waitin' out on de aidge or de crowd. 'spectin' sumpin gwine happen. fur he knowed de ways er Mistah $\mathrm{Dog}$.

"Fer so long time ol' Wolf went risitin' roun' de naberhoods 'lowin'. 'Tar! I done tol' you Mistah Dog wan't dairl! I done tol' you so! Mel,he nex' time rou pay me some 'tention w'en I talk.' He kep' on dat-a-way 'twel folks got plumb wo' out wid him. an' dey'd git outen de way w'en dey seed him comin'. I tell you. folks, dat sort er talk mek a man mighty onpop lous. I done study 'bout hit a heap an' I mek up my min' dat's huccome some er de prophits ter git stoned in de ol' days; fus' dey tol' de people, 'Dis thing gwine happen,' an' den w'en hit come ter pass dey go roun' sayin', 'Th-huh! ain' we-all done tol' you so?' Seem lak folks jes' kain't stan' dat. nohows: "pears lak we ain mare so s.t we kin: de flaish too weak, an' dem ar prophits orter knowed dat." 


\section{HOW THE DEER LOST HIS UPPER TEETH}

Aunt 'Phrony followed up the last story with one which dealt with that tricky little fellow, Mr. Hare. "You ehillen," she eommenced, "mebbe kin 'member, an" den ag'in mebbe you kain't, de tale I done tol' you onct 'bout de ereeturs teckin' 'way de hawns f'um ol' Hyar' an' puttin' 'em on Mistah Deer's haid. Well, den, Hyar' he bin a-studyin' an' a-studyin' how he gwine git eren wid him fer dat. So one day w'en he wuz settin by de road. gnorrin' on de bark ur a black locus', 'long come Mistah Deer jes' a-lopin' by. Hyar' call out 'howdy' to 'im, an" he stop and pass de time er day. He see Hyar' jes' a-gnorrin' 'way an' he say, he do. 'Lawsy, Mistah Hyar'. w'at pow'ful fine toofs you got. Huecome dey so sharp?'

"Hrar' he say, sczee. 'Thanky, suh, my toofs is right good and sharp, an' dis hueeome dey so, dey ain' grow dat-a-ray; I oon have "em of I did n' wu'k fer em; I done whet 'em myse'f er dey ain' be dis-a-way. Jes' look at me gnor dis bark. Lemme see yo" toof:, suh.'

"Deer he putt down his haid an" open his mouf wide, an' Hyar' he tueken him by de tongue an 'peer inside, lak he might'ly consarned 'bout de state er Mistah Deer's toofs. He putt on a sollum look an' shuk his haid an' say, sezee, 'Mry! my! Mistah Deer, yo' toofs is in a pow'ful bad way. I dunno 't all how rou mek out ter chaw ro' vittles; you mus' hatter gobble 'em down hull; I boun' 


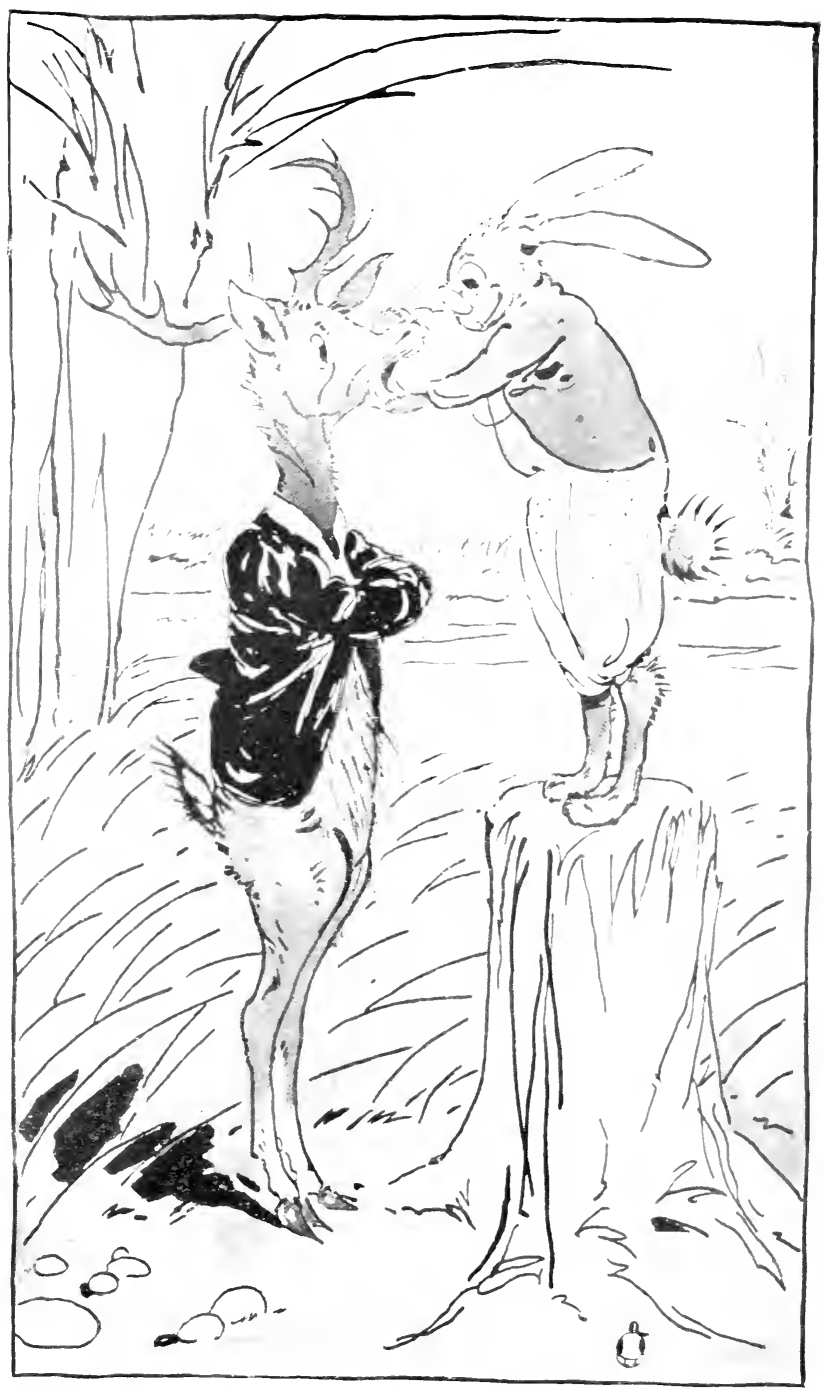

"MISTAH HEER, Y" TOUFS IN IX A POW"FIL BAD WAY" 



\section{HOW TIIE DEER LOST HIS LPPER TEETH}

ef de trufe wuz knowed, yo' stummick all out er order. You better lemme fix up dese toofs fer you 'fo' dey gits any wusser 'n w'at dey is; better let me whet 'em fer you.'

"Deer he "low dat he have bin feelin' right squawmish in de stummick jer lately, an' he 'low, he do. 'I ain' bin blamin' hit on de toofs, but I reckon you'se right. an' ef you kin fix me up wid a outfit er vittle-'stroyers lak yo`n, jes' you go ahaid an' do hit, quick 'z you kin.'

" 'All right,' sez Hyar, sezee. 'I'm yo' man; I gwine fix you up so ‘'t you'll sprise yo se'f an' all yo' fambly, an' 'twon't be long "fo" rou"ll fin' yo'se f so fatted up dat li'l mo' an you'd come bustin' thu yo' hide.' Wid dat he go off an" git him a rock an' den he come back an' say, 'Tow, ol' man, jes' lay yo' haid back an' open yo' mouf wide an' gimme good elber-room an' I'll have you all whetted up in a jiff, so sharp "t you kin bite tenpenny nails in iwo ef you wanter.'

"Deer he "low, 'Oh, lawsy! Mistah Hyar', I'se 'feard you grwine hu't me. deed I is!"

"Fraidy-calf!" sez Hyar'. sezee, 'how you reckon I gwine hut you wid dis li"l ol' no-kyount rock. But I'll jes" g'long an' leave you wid dem misable stumps in yo' mouf. ef you sesso, dough I had hit in min ter fix you so`s't you cu'd mek yo' livin' heap easier dan you does now.'

"Deer he feel kind er 'shame' ur hisse'f an' he 'low. 'Oh. go ahair. suh; reckon I ain' feard ur a li'l thing lak dat: I bin rumnin' roun' thu dese moods 'mongs' de bars an de wil'ats an de wolfs too long ter be 'feard er wat a li"l feller lak you kin do ter me. Go on wid de grindin"' 


\section{AT TIIE BIG IIOLSE}

"Itrar" he chum up on a stump whar he cuid reach good, an' den he jes" rub an' rub an' rub on Mistah Deer's upper toof's. I"en he got 'em groun' half-way down. Deer he think hit 'mence ter feel mighty queer an' he say, sezee. "Look-a-yer, Mistah Hyar', w'at you doin' to me? I 'clar" dat don' feel right, 'deed hit don'.'

" 'Is dasso:" Hyar' 'low. 'T"y I is sprise'. But nemmine, you gwine feel all right torreckly. I'se jes' 'mencin ter git a nice aidge on yo' toofs, an" w'en I'se thu wid you, you kin gnor de bark offen de saplin's an' git yo"se'f sump'n ter eat sidesen dish yer triflin' moss you bin livin' ofl all dis time. I gwine fix you up so"st you lin gnor anything you 've a min' ter.'

"Deer he "low, "Tame er gracious! go on, den, in fer a penny, in fer a poun"! but don' be long 'bout hit, fer I mighty nigh wo" out." He kep" gruntin' an' groanin' an 'screwin'. wid de col' shiver's rumnin' up an' down his back, an' Hyar" he kep" on grindin' an' grindin' 'twel po' ol' Deer ain have nuttin' but de stumps ur his upper toofs lef' in his haid.

Den Hyar he "low, 'Dar you is now, Nistah Deer, right ez a trivet, suh; jes' g'long an' gnor on de bark er dem saplin's yonner, an I boun' you you'll be s'prise' ter fin ” how easy 'tis.'

"'Thanky, suh, all right, suh," sez de Deer, sezee, 'I sut'n'y is "bleeged ter you. I bin wantin" me sump'n diff'nt ter eat dis long time,' an' wid dat he walk over ter de saplin's.

"Hrar" he 'mence sneakin" an' slidin" off, but he ain' go fur; he hide behime a tree an' watch ter see w'at Deer grine do. II' $\mathrm{en}$ Deer fine out he kain't gnor de bark 't all, an' dat his upper toofs mos' all gone, he jes' stomped 


\section{HOW TIIE DEER LOST IIS TPPER TEETII}

his hufs an' r'ared 'roun'. "Trufless li'l bumnel er trash!" he say. 'I wish t I had "im yer on one'r dee haws er mine dis re'y mimnit. I boun" you I'd show "im huecome! Crone an done me outer my toofs. wirl nuttin' lef" but a ache in place ur "em." an" wid dat he shuk his hawns an' jes' r'ared 'roun' "twel IIvar" wuz mos" feard ter show hisse"f.

"Las he cuidten keep back his impence no longer. so he stick his haid out an' sar. 'Hi! yi!' Mistah Deer. you "pears ter be kind er store up bout de toofs. of I ain" mek no misteck. Ain' dat a pity! sho"! sho"! I'se sprise" you trus" me wid dat job, I sut'n Y is, kase you moughter knowed I wuz gwine git even wid you fer wearin dem hawns de creeturs tucken "way fom me. w" en yon knowed dey wuz mine by rights. I reckon you done ferwot how you drug me befo de krouncil. a prisner. but I tell you, suh, I ain' fergit hit: I bin waitin' all dis time ter git eren, an' I low dat I done come up wid rou. fer folks kin do widont hawns. but dey kain't git long re"y well widout toofs. lesen der teck ter swollorin'soprittles. Ain' dat de trufe؛ I leare hit ter rou, sulh, "kase I knows dat you in a fix ter know all bout hit.'

"Wid dat he let nut a big hoot an' step" off home widout waitin' ter look hehime. an' lef" ol Deer chawin' off de li"l buds an young twigs, an' dat w'at de deers bin doin" urer sence. "kase dey ain' got no upper toofs wuf speakin' ur ter do der chawin' wid." 


\section{TIIE IIARE DISAPPEARS FOR EVER}

"Lmph!" said Eliza, when she had heard how the Deer came to have such short upper teeth. "Umph! I know Deer felt bad widout dem toofs: I know he did, 'kase I done los' my own, an' dish yer thing ur gommin' hit ain "w'at hits cracked up ter be, 'deed hit ain'. Hit don' sprise me dat Deer wuz mad wid ol" Hyar'. I reckon dey kep" mighty cle"r ur one nu'rr atter dat "spe yunce."

"Deed dey ain', den," said Aunt 'Phrony. "Co'se Hyar" he try ter keep outen de way fer a li'l w'ile, but Deer he let on ter be fren 'ly an' familious, waitin' fer de chanct ter git eren wid him, an' dat th'ow Hyar' offen his gyard, an' he git so, pres'n'y, dat he ac' jes' 'z dough nuttin' done happen 'twix' 'em. Hit run on lak dat ontwel one time w'en Hyar" wuz gwine roun' tendin ter be a doctah an "meddlin' an" muddlin" wid de sick folks.

"One day a baby git scalted wid hot water an' go ter crrin' an' hollerin so dey kain`t do nuttin' wid him. Hyar' he come dat way an' he say, he do, 'Hi! w'at on yearf is de marter wid dat chil"? He howl wussern any ol' wolf on de mountain; done split my years f'um top ter bottom.'

"Dey tol" 'im w'at wuz de marter wid de chil', an' he say, sezee, 'Jes' lemme see dat chil'; I'se a doctah, an' I boun' you I kin gin him sump'n dat 'll ease de pain an' mek him stop cryin', fer I tell you p "intedly dat I'se a fuss'-class doctah, an ef any un you feelin' kind er 342 


\section{THE HARE DISAPPEARS FOR ETER}

doncey he better insult me 'bout hit right now, 'kase I dumno w'en he gwine git de ehanct ag in.'

" "Well,' dey ses ter him, 'w'at kin you do fer burns?' "'You lemme 'lone fer dat,' sezee. 'I does hit wid water, but ef I wuz ter tell you jes' how, de chawm done be bruk. You mus' putt me in de house an' den shet de do" an' fill up all de chinks an' den go "way a li"l piece, fer I mus' be so't eye kain't see ner year lissen at me.'

"'All right,' dey ses, an' den dey went ter w'uk fillin' up de chinks, dobbin' 'em wid clay, an' w'ile dey 'z doin' dat Hyar' he sa'nter roun' kind er kyarless an' foun' a li'l hole whar he cu'd git out ef anything wuz ter happen, 'kase he seed de baby wuz nigh mos' daid an' he'z 'feard dey mought blame him fer hit. ' $\mathrm{y} y, \mathrm{y}^{\mathrm{y}}$ !' he sez ter hisse'f, 'I didn' s'pose de chil' wuz dat bad off er I oon 'a tol' 'em I wuz a doctah. I wish I wuz safe outer dis, but I gotter go thu wid hit now. $M y$ ! my! dis shows me I better stick ter de trufe atter dis.'

"Tell, de folks got de dobbin" done an' den dey" walked 'way f'um de house a piece ter see w'at gwine happen. Hyar' he tuck an' tuck de baby an' soused hit in de water an' de chil' gin one onyearfly yell an' den wuz still, an' Hyar' he see dat 'twuz plumb daid, an' he say ter hisse'f, sezee, 'Come on, Doctah Hyar', le's we-all git outen dis in a hurry ef we know w'at good fer us.' an' he ga'rr hisse'f toge'rr ter spring out er de hole.

"Nor, de mammy er de chil' she done stay behime ter lissen, an' w'en she year de baby yell she call ter de res', 'Come yer, quick, all er you! hurry up fas' 'z you kin! Dat owdacious hypermoerit done kill my chil'. I know he have! Bus' in de do', yout-all, an' nab him 'fo' he kin 343 


\section{AT THE BIG HOUSE}

git off. Ef he done hurt my chil' I ain' gwine leare one spear er hya'r on his misable hide! You year me now!

"So de folks come a-runnin' an' bus' in de do' an' foun' de chil' layin' dar daid. Hyar' he run outen de hole, an' de ooman she set de dogs on "im an' dey chased him inter a hole in de tree an' stood dar keepin' gyard ontwel de ooman come up. De people rushed up, yellin' an' callin', an' de dogs wuz darnsin' roun' yappin' an' yowlin', an' de ooman wuz cryin' an' kyar'yin' on an' bellerin', 'Oh, lemme git at him; jes' lemme putt my han's on him onct an' he'll wish he'd died 'fo' he wuz bawned!' Ol' Hyar' think his time done come fer sho'.

"De ooman tucken a stick an' twis' hit roun' in de tree, an' she say, sez she, 'Cm-umph! got you now, mis'able li'l thief-an'-body-snatcher! Atter you wid a sharp stick, sho' 'nuff. Gwine git you dis time!' Las' she twis' him outen de tree an' he fall right inter her lap. She snatched him by de scruff er de neck an' 'gun ter lamm him fus' on one jaw an' den on tu'rr, talkin' at him all de time, 'Till my baby, will you! Meddle wid de doctah's trade, will you! Play de torm-fool wid us all, will you! Teck dat, now! an' dat! an' dat!'

"De ooman she done skunt one'r his laigs wid de stick, an' hit 'mence ter smart him right much, so he say, mighty meek an' numble, 'Please, ma'am, fer ter let me down a mimnit w'ile I fix my laig; hit done smart me lak a hull nes'ful er wast-es. Kill me ef you gotter, but, fer de lan' sake, lemme fix dis laig fus'!'

"De ooman wuz fool 'nuff ter let him down, an' I boun' ter let you know dat he did'n' let de grass grow unner his footses; not him. He wuz off an' hid 'way down in de woods in a kvave quicker'n I kin tell hit. 


\section{THE HARE DISAPPEARS FOR EVER}

"De onman cu'dden tell how in de name er goodness she gwine git eren wid him. so she call all de folks toge ${ }^{\circ}$ an ax "em fer ter he p tell her how ter do hit. W'iles dey wuz talkin' 'long come de Deer, an' year w'at dey sar. an' he sez. sezee, 'Sho'! dat's easy ez rollin' offen a log. Leare dat ter me: I'se de man whar kin fix up dis job. W' Y. I kin sen' dat feller clean orer de ocean, so fur he nurer kin git back ag in, ef you sesso.'

"De folks hilt a li"l confab an' mek up der min's dey bes' let Deer git redd er de Hyar'. 'Deed dat I will,' sezee, an' he went sa'nterin' 'long, jes' ez gaily ez you

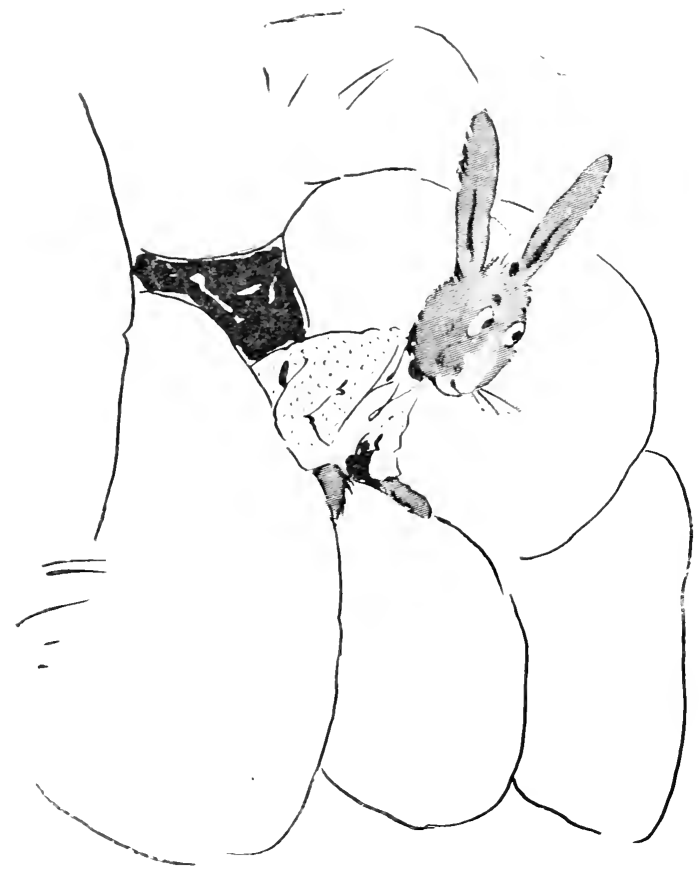




\section{AT THE BIG HOUSE}

please down inter de woods. Hyar' yearn him comin' an peeped outer de kyave ter see who 'twuz. W'en he see "twuz Deer he mek up his min' ter come out an' pass de time er day wid him, 'kase he thought Deer wuz his fren' an' wuz fool 'nuff ter s'pose Deer done fergot all 'bout harin' his toofs groun' down. So he come prancin' out, jes' ez peart ez a lizzud.

"Deer he say, 'Howdy, ol' man; whar you bin keepin' yo"se'f all dis time? Mrus"n" hide yo"se"f "way f"um yo" fren's; dey jes' natchelly kain't git 'long widout you. I come ter ax you ef you oon lak ter go "long er me an" teck a li'l walk dish yer fine day. Do you good; you look kinder pindlin:'

"Hyar' kind er tired er stayin' in de kyave all 'lone. so he say. 'Dat I will! I'se mo' tickelt dan a b'ar wid a bee-gum ter see you onct mo'. Pow'ful lonesome, suh, roun' dese diggin's, pow'ful lonesome; dis de fus' time I uver 'spicioned dat I wuz n't good comp'ny:'

"Dey went romantin' thu de woods an' pres'n'y dey come to a branch, an' Deer he say ter ol' Hyar', 'Tell. now, my soople-jack fren', does you think you kin jump dish yer?"

“'Dat I kin,' sez Hyar', sezee, 'a li'l ol' narrer stream lak dis; dis nuttin' 't all. Kin you?'

" 'Oh, I reckon I mought mek out ter spraddle 'cross somehows,' sez Deer, 'le's we-all step back a piece an' gin a run an' a spring toge'rr an' see w'icht kin cle'r de branch bes',

"'All right,' sez Hỵar, sezee, an' wid dat dey run to'des de branch. Hyar' he gin a monst'ous jump dat lan' him clean on tu'rr side. but Deer ain' jump 't all, jes' stan' at de aidge er de branch laughin'. Hyar' he $3 \pm 6$ 


\section{THE HARE DISAPPEARS FOR EVER}

baig him f"um tu'rr side not ter be 'feard but ter ga'rr his laigs toge'rr an' jump lak he have some sense an' not stan' dar grimnin lak a plumb ijit.

"De mo" he baig de mo' Deer stan' still, an' pres'n'y de branch 'mence ter git wider an' wider 'twel hit look 'zackly lak de ocean, an dat's w'at 'twuz, sho' 'nuff, fer Deer was a mighty big cunjerer in dem dars, an' he done made de ocean look narrer ez a branch, so's't he cu'd fool ol Hyar. W"en he got him across he tucken de spell offen Hrar"s eves an' let him see dat he done cross orer de ocean an' kain't git back ag.in, an' dar whar he bin urer sence, dat ol'-time mischief-mekin' Gre't Hyar'. Dish yer w'at we have now is jes' a li'l no-kyount, harmless kin er his'n. an' all dat's lef' er de ol' doin's is jes' dese yer tales wat I bin tellin' you. But dish yer's de las' ur him, 'kase I done sont him clean 'cross de big water, so I kain't tell you no mo' ur his doin's, eren ef you wuz ter be yer ter year me tell 'em, w'ich dat you won' be: you givine be fur 'nuff 'way f'um we-all an' all yo' kinry;" and here the old woman threw her arms about the children and led the rery sincere chorus of regret from these humble friends who had added so much to the pleasure of the risit and whom they were not to see again. For the old plantation was sold not many years after, and their mother never went back.

When the children were grown they wandered once more to the spot which had been the home of their ancestors for more than a.hundred rears, but all was changed. all the faces unfamiliar, and Aunt 'Phrony and Aunt Nancy lay peacefully beneath the spreading trees of the old negro burial-lot back of the garden, where so many faithful black heads had been laid in the 


\section{AT THE BIG HOUSE}

days that were gone,-those unreturning days when between the white man and the black were confidence and esteem and faith and trust and affection. As they turned away from the spot they sighed regretfully for these things, gone as utterly and surely as Molly Hare herself has ranished from the firesides of her once cordial hosts, banished by the arts of a powerful conjuror who performs many wonderful feats and incantations through the agency of twenty-six nimble little characters, known as the letters of the alphabet. 



4
$\vdots$
$\vdots$

2

,

$\therefore$ 


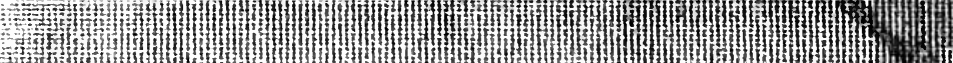

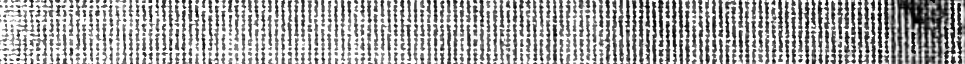
(10)

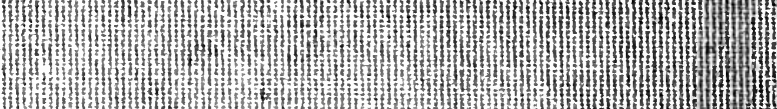
H.1. 15.

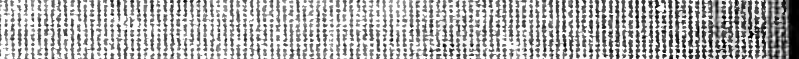
10
10

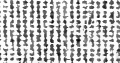

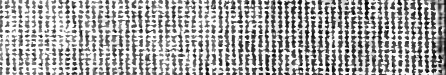

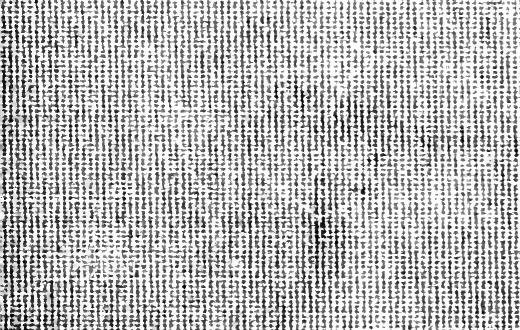

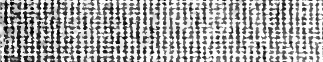

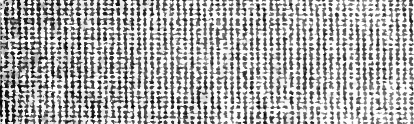

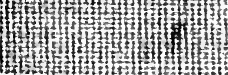

